\title{
Mind over food
}

Citation for published version (APA):

Franssen, S. H. M. J. (2021). Mind over food: The influence of mindset on brain, body and behaviour. [Doctoral Thesis, Maastricht University]. Maastricht University. https://doi.org/10.26481/dis.20210430sf

Document status and date:

Published: 01/01/2021

DOI:

10.26481/dis.20210430sf

Document Version:

Publisher's PDF, also known as Version of record

\section{Please check the document version of this publication:}

- A submitted manuscript is the version of the article upon submission and before peer-review. There can be important differences between the submitted version and the official published version of record.

People interested in the research are advised to contact the author for the final version of the publication, or visit the DOI to the publisher's website.

- The final author version and the galley proof are versions of the publication after peer review.

- The final published version features the final layout of the paper including the volume, issue and page numbers.

Link to publication

\footnotetext{
General rights rights.

- You may freely distribute the URL identifying the publication in the public portal. please follow below link for the End User Agreement:

www.umlib.nl/taverne-license

Take down policy

If you believe that this document breaches copyright please contact us at:

repository@maastrichtuniversity.nl

providing details and we will investigate your claim.
}

Copyright and moral rights for the publications made accessible in the public portal are retained by the authors and/or other copyright owners and it is a condition of accessing publications that users recognise and abide by the legal requirements associated with these

- Users may download and print one copy of any publication from the public portal for the purpose of private study or research.

- You may not further distribute the material or use it for any profit-making activity or commercial gain

If the publication is distributed under the terms of Article $25 \mathrm{fa}$ of the Dutch Copyright Act, indicated by the "Taverne" license above, 


\section{MIND OVER FOOD}

The influence of mindset

on brain, body and behaviour

Sieske Henriëtte Margriet Jessica Franssen 
ISBN: 978-94-6416-385-8

Cover design: Liesbeth Wilmer

Lay-out: Publiss |www.publiss.nl

Print: Ridderprint | www.ridderprint.n|

(c) Copyright 2021: Sieske Henriëtte Margriet Jessica Franssen

The research presented in this thesis was financed by the Netherlands Organization for Scientific Research (NWO) Vidi-grant (452.16.007) awarded to Anne Roefs, Vici-grand (453.10.006) awarded to Anita Jansen and FBC-grant (057.13.010) awarded to Anita Jansen and the Maastricht University Interfaculty Program 'Eatwell'.

All rights reserved. No part of this publication may be reproduced, stored in a retrieval system, or mechanical, by photocopying, recording, or othewise, without the prior written permission of the author. 


\section{MIND OVER FOOD}

The influence of mindset

on brain, body and behaviour

\section{Proefschrift}

ter verkrijging van de graad van doctor aan de Universiteit Maastricht,

op gezag van de Rector Magnificus, Prof.dr. Rianne M. Letschert

volgens het besluit van het College van Decanen,

in het openbaar te verdedigen

op vrijdag 30 april 2021 om 12.00 uur

door

Sieske Henriëtte Margriet Jessica Franssen 


\section{Promotores}

Prof. dr. A.J. Roefs

Prof. dr A.T.M. Jansen

\section{Beoordelingscommissie}

Prof. Dr. R.W. Goebel (voorzitter)

Prof. Dr. S.E. la Fleur (University of Amsterdam)

Prof. Dr. D.E.J. Linden

Prof. Dr. E.F.C. van Rossum (Erasmus University Medical Center Rotterdam)

Prof. Dr. A.M.W.J. Schols 


\section{Table of contents}

$\begin{array}{lll}\text { Chapter } 1 \text { General introduction } & 7\end{array}$

Chapter 2 Power of mind: attentional focus rather than palatability 23 dominates neural responding to visual food stimuli in females with overweight

Chapter 3 Effects of mindset on hormonal responding, neural representations, subjective experience and intake

Chapter 4 Your mind on sweet taste: Effects of anticipated caloric load 93 on metabolic response to carbohydrate consumption

Chapter 5 Neural correlates of food cue exposure intervention for 109 obesity: a case-series approach

Chapter 6 Summary of main findings and general discussion

References

Samenvatting

Impact addendum

Dankwoord 


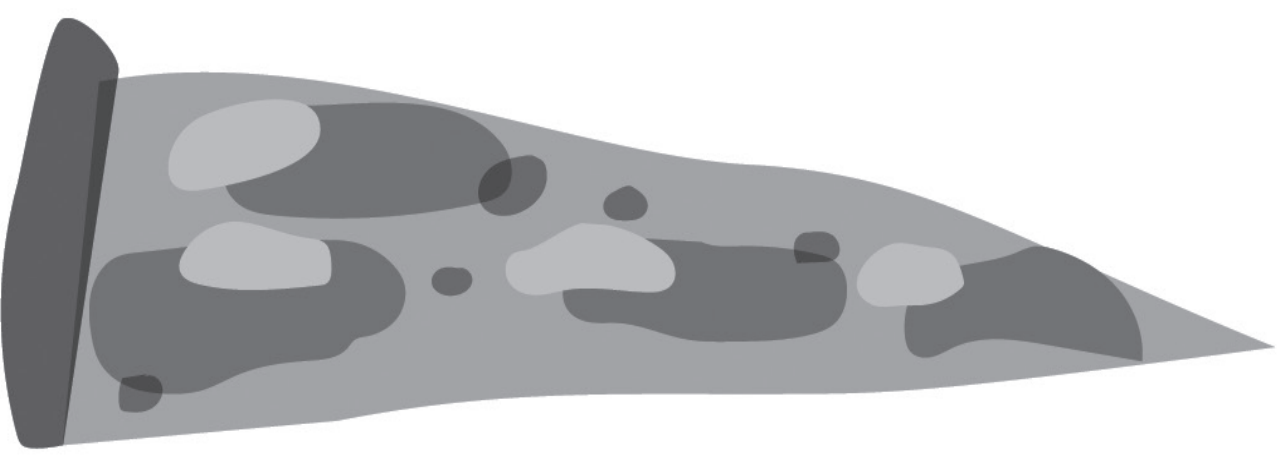


CHAPTER I

GENERAL INTRODUCTION 
Eating behaviour is a highly relevant topic, as everyone needs to eat and drink to stay alive. There are several cultural, psychological and physiological factors that contribute to what, when, how, and how much someone eats. Of course, one of the main reasons to eat is to alleviate homeostatic hunger (Morton et al., 2006; Murphy et al., 2006). The main physiological contributors to the regulation of eating behaviour are the brain and the gut. Hormonal peptides released from the gut provide information about energy needs to relevant brain areas that are involved in the homeostatic control of food intake. This connection between brain and gut is also referred as the gut-brain axis (Gibson et al., 2010; Hussain \& Bloom, 2013). Brain areas that have been associated with food processing are amygdala, hippocampus, ventral pallidum, nucleus accumbens and striatum, anterior cingulate, orbitofrontal cortex (OFC), insular, posterior fusiform, (ventro)medial and dorsolateral prefrontal cortices (vmPFC and dIPFC) (Berthoud et al., 2011; Haber \& Knutson, 2010; van der Laan et al., 2011). Two of the main hormones involved and interacting with neural processing in regulating hunger and satiety are ghrelin and GLP-1 respectively (Hussain \& Bloom, 2013).

One might now be tempted to conclude that eating behaviour is simply the result of homeostatic hunger. From an evolutionary perspective, one should indeed eat as a response to homeostatic hunger, respond to one's bodily caloric needs. However, an important other reason that people eat is so-called "hedonic" hunger. When one experiences hedonic hunger, it is not the caloric need that drives food intake, but the pleasure derived from food consumption (Appelhans, 2009; Lowe \& Butryn, 2007). Note that caloric content and palatability are strongly associated. In the current society, there are plenty of such highly rewarding foods, which are mostly rich in sugar and fats. When eating more calories than one needs, one gains weight. The combination of these high-caloric palatable foods being omnipresent and the fact that people do not need to move much, results in an environment in which weight is easily gained, an obesogenic environment (Swinburn et al., 2011).

If people excessively engage with the current obesogenic environment by overeating high-caloric foods, then they are at risk of becoming obese. Being overweight or obese is associated with severe negative health outcomes, like several chronic diseases, diabetes, cardiovascular diseases and even certain types of cancer. Additionally, being overweight is associated with mental illnesses such as depression and eating disorders (American Psychiatric Association, 2013; Baumeister \& Härter, 
2007; Scott et al., 2008). Today, obesity has reached pandemic proportions, with more than 1.9 billion adult people being estimated to be overweight or obese, and these numbers are still rising. Also, these high obesity numbers have a huge economical and societal impact (Afshin et al., 2017; World Health Organization, 2020)

The major cause of gaining weight and becoming obese is a prolonged energy imbalance, with the number of consumed calories exceeding the number of expended calories (Hall et al., 2012). The question arises how this positive energy balance develops and maintains. Genetics and environmental influences and their interaction are considered important here (Bell et al., 2005; Hill, J. O., Peters, 1998; Hill et al., 2000; Silventoinen \& Kaprio, 2009). Indeed, about $67 \%$ of the variability in BMI has a genetic basis. But the inherited variability in BMI is mainly caused by behaviour; regulation of food intake and volitional activity (O'Rahilly \& Farooqi, 2006; Ravussin \& Bogardus, 2000). Although in the Western world everyone is living in the same obesogenic environment, not everyone is obese. It has been proposed that overweight and obese people may be more sensitive to the current obesogenic environment. Indeed, research has shown that overweight and obese people show an increased high-caloric food-cue reactivity to cues that predict food intake (Boswell \& Kober, 2016). This increased food-cue reactivity makes it more difficult to refrain from consuming those high caloric foods (Boswell \& Kober, 2016; Jansen et al., 2015).

However, people are unlikely to respond to the obesogenic environment always in the same way. Just imagine a woman who is at her favorite restaurant on a Saturday night after a week of hard work. She will likely enjoy the foods that are severed and be mostly interested in the tastiness of the foods. Now imagine that she just tried on new tight-fitting jeans, walked by a gym with posters of very fit people, and then goes grocery shopping. How would she look at the abundance of snack foods now? She might be much more concerned with health and body weight when she selects her groceries. In the first situation, the focus is on eating enjoyment and one could say that the woman is in a hedonic mindset, whereas in the second situation, the focus is on health and body weight, and one could say that the woman is in a health mindset.

The central topic of this dissertation is to examine the influence of mindset on psychological and physiological variables involved in eating behavior. Mindset will be operationalized in this dissertation as: loss of control versus control mindset, hedonic attentional focus versus neutral attentional focus, and a high-versus mid-versus low- 
caloric label on to be consumed sweetened beverages. Responses to food stimuli will be examined by assessing food intake and subjective experiences (e.g. food cravings, hunger, self-control), neural responses (in mesocorticolimbic system), gut-hormones reflecting hunger and satiety (respectively ghrelin and GLP-1) and metabolism (resting energy expenditure). In addition, and as a possible intervention for decreasing food cue reactivity and overeating, we examined the effect of food cue exposure therapy on neural responses to food. First, these measures (see Figure 1 for an overview) and prior research on mindset, brain, body and behavior will be introduced, after which an outline of this dissertation is provided.

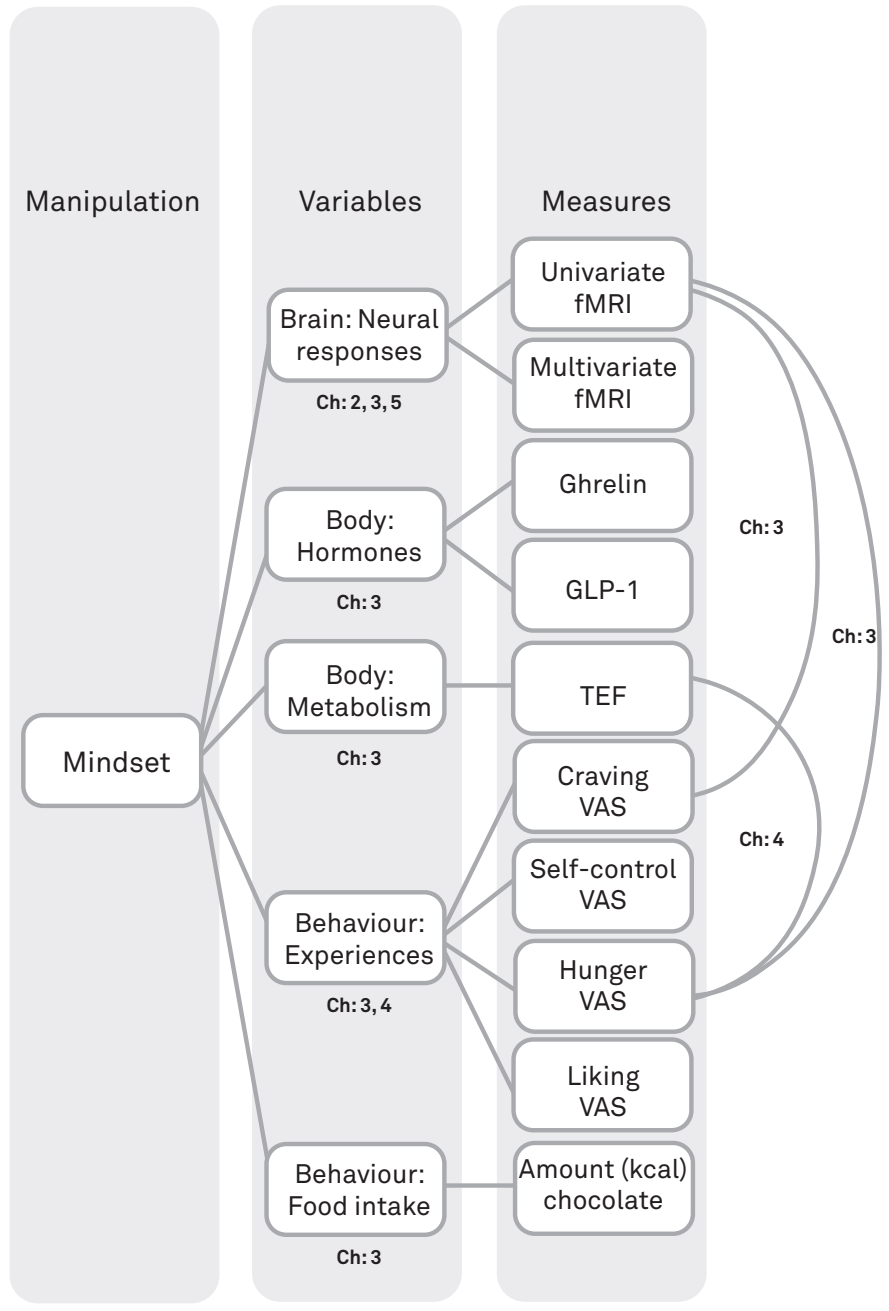

Figure 1. Manipulation, variables and measures and their relationships assessed in this dissertation. Abbreviations: $\mathrm{CH}=$ chapter, fMRI = functional Magnetic Resonance Imaging, GLP-1 = Glucagon-like Peptide 1, TEF = Thermic Effect of Food, VAS = visual analogue scale. 


\section{Measures of brain, body and behavior}

\section{Food intake}

In the research for this dissertation, eating behaviour is assessed in a laboratory setting with a so-called bogus taste test. During a bogus taste test participants think that the goal is to assess taste perceptions, whereas actually the amount of (caloric) intake is measured. The bogus taste test has been suggested as a valid measure to assess eating behaviour (Robinson et al., 2017).

\section{Subjective experiences}

Subjective experiences, like the feelings of hunger, satiety, liking, craving and feelings of self-control are assessed in several studies as well. These subjective measures could be related with food intake as well as hormonal or neural responses.

\section{Neural responses}

The most widely used method to study neural responses to food stimuli is functional Magnetic Resonance Imaging (fMRI). With fMRI, neural activity during task performance can be measured. Neural responses to food stimuli are thought to be mainly located in the dopaminergic mesocorticolimbic system (Burger \& Berner, 2014; Leigh \& Morris, 2018; Volkow et al., 2013; Volkow et al., 2011). This system is also referred to as the reward system, because it is thought to be involved in processing pleasure and reward (Volkow et al., 2011). Brain areas of the mesocorticolimbic system include the amygdala, hippocampus, ventral pallidum, nucleus accumbens and striatum, as well as the anterior cingulate, orbitofrontal cortex (OFC), insular, posterior fusiform, (ventro)medial and dorsolateral prefrontal cortices (vmPFC and dIPFC) (Berthoud et al., 2011; Haber \& Knutson, 2010; van der Laan et al., 2011). From these areas, the anterior cingulate, dorsolateral, and medial prefrontal cortices are also being considered to have a regulatory function in neural food-cue reactivity (Giuliani et al., 2018).

\section{Gut-hormonal responses}

Several hormones are involved in the regulation of hunger and appetitive motivation. One of these hormones is ghrelin, which is referred as the "hunger hormone". Ghrelin is secreted from the endocrine cells of the stomach (Kojima et al., 1999) and binds with the brains' receptors in the arcuate nucleus and ventromedial hypothalamus to regulate appetitive motivation (Baynes et al., 2006; Morton et al., 2006; Murphy 
et al., 2006). Ghrelin release is regulated by energy and nutrient sensory processing of the gastrointestinal tract; when energy intake is low or the stomach is empty, ghrelin release is increased, and when energy levels are higher, ghrelin secretion is suppressed (Baynes et al., 2006; Murphy et al., 2006). Another endocrine hormone involved in appetitive motivation is glucagon-like peptide 1 (GLP-1). GLP-1 is secreted from the small intestine and colon into circulation, signaling the pancreas to release insulin and inhibiting glucagon release (Drucker, 2006). GLP-1 producing cells are also located in the solitary tract nucleus of the brainstem with projections throughout the brain (Schwartz et al., 2000; Vrang \& Larsen, 2010). As GLP-1 also is known to enhance satiety feelings and inhibit appetite, it is also referred to one of the gut 'satiation hormone' (Dailey \& Moran, 2013; Flint et al., 1998).

\section{Metabolic responses}

In addition, our bodies' metabolic system contributes to food processing by physiological responding to food intake with its accompanied energetic caloric loads (e.g., sugar and fat) to digest it. The metabolic response can be examined by measuring the resting energy expenditure (REE) before and after consuming food or a beverage. By subtracting the pre- from post-consumption REE, the so-called thermic effect of food (TEF) is calculated. This TEF represents the energy required to process and metabolize this consumed food/beverage (Reed \& Hill, 1996).

\section{Prior research on mindset, brain, body and behavior}

\section{Food intake and subjective experience}

For a long time, it has been known that expectations can affect eating behaviour; when expecting to consume more calories participants subsequently ate less and reported an increased satiety as compared to expecting less calories (Wooley, 1972). Other studies have shown that beliefs can influence high caloric food consumption by using health claims or caloric content labels (Faulkner et al., 2014; Gravel et al., 2012; Hogenkamp et al., 2013; McCann et al., 2013; Roefs \& Jansen, 2004; Shide \& Rolls, 1995). For example, caloric intake is reduced when consuming food with a perceived higher caloric content and caloric intake is increased when consuming food with a perceived lower caloric content (Faulkner et al., 2014; Gravel et al., 2012; Hogenkamp et al., 2013; McCann et al., 2013). In addition, it has been shown that labels on food information (i.e. healthy or hedonic labels) can influence liking and 
perceived healthiness (Provencher \& Jacob, 2016). When applying cognitive strategies by upregulation of craving healthy foods and reappraisal unhealthy food, it has been found to be possible to upregulate craving for low calorie foods and to decrease consumption of high caloric food intake foods (Reader et al., 2018). These studies suggest that expectations or rethinking the value of food can influence behaviour towards foods significantly.

\section{Neural responses}

It has been proposed that overweight people show an increased neural anticipatory reward response to food stimuli (Leigh \& Morris, 2018; Eric Stice et al., 2013). Several functional magnetic resonance imaging (fMRI) studies showed increased activity in one or more reward-related brain areas when viewing food stimuli in participants who are overweight or obese as compared to lean controls (Frankort et al., 2012; Pursey et al., 2014; Rothemund et al., 2007; Stoeckel et al., 2008; Yokum et al., 2011). Some studies found that this increased reward-related activity was specifically increased for high-caloric foods, as compared to low-caloric foods (Rothemund et al., 2007; Stoeckel et al., 2008). However, other studies showed either reduced or no significant differences in neural reward related activity in response to visual food stimuli in people with overweight versus healthy-weight (Brooks et al., 2011; Frankort et al., 2012; Schienle, Schäfer, Hermann, \& Vaitl, 2009; Stice, Yokum, Blum, \& Bohon, 2010). So, the diversity in research findings is too large to draw any firm conclusions regarding the role of neural reward responses in obesity. Ziauddeen and colleagues (2012) clearly discuss this large degree of inconsistency that is present in the neural food-reward literature on obesity. Here, it was stated that the involved brain-regions as well as the direction of effects varied highly over studies, which led the authors to conclude that "... the pattern emerging from studies comparing obese individuals and binge-eaters with controls is most remarkable for its variability and inconsistency "(p.283) (Ziauddeen et al., 2012). Likewise, a recent review also emphasized the inconsistency of evidence for the dopaminergic system involvement in the neurocognitive profile of obesity (Janssen et al., 2019). Taken together, the idea of increased anticipatory reward neural responding in people with overweight as compared to healthy weight is not supported by solid consistent empirical evidence.

Many previous fMRI studies used passive viewing designs. During such a passive viewing paradigm, participants view food stimuli without specific instructions and are not asked to perform any stimulus-related task. When interpreting results, it 
is assumed that people automatically focus on the palatability of high-caloric foods (Martin et al., 2010; Rothemund et al., 2007; Stoeckel et al., 2008; Thomas et al., 2015). Conclusions drawn from this literature seem to depend mostly on the logic of reverse inference: "to infer the likelihood of a particular mental process $\mathrm{M}$ (i.e., reward responsiveness) from a pattern of brain activity $A$ (i.e., activity in the mesocorticolimbic system)" (Poldrack, 2011) (P. 693). Important is that M in unknown in a passive viewing paradigm because the researcher does not know how the participants process the presented food stimuli. Also, the mental process of the participant may fluctuate over the course of the scanning session. Therefore, one cannot conclude that a food stimulus is rewarding based on the observed neural activity. So, it is important to be certain about the ongoing mental process to adequately interpret an observed pattern of neural activity.

People may actually not (only) focus on the palatability of food, but (also) on the health value of food, and they may be alternating between those frequently (Roefs et al., 2018). As people who are overweight often have a dieting history and score relatively high on measures of dietary restraint (Herman \& Polivy, 1980), they may be especially likely to alternate their focus of attention between evaluating palatability and health value of high caloric foods. Importantly, alternating between these attentions may happen - unbeknownst to the researcher - within and across participants as well as within and across studies. This double-sided nature of high caloric foods may be one of the causes of the inconsistency in the literature on neural food reward processing in obesity. Both the hedonic and the health value can affect the neural responses to food stimuli, and whether the hedonic or the health value drives the neural response might depend on a person's cognitive or emotional state or on the current situation (Roefs et al., 2018).

Several fMRI studies showed that mindsets, like expectations and task demands, affect neural responses to food stimuli (Bhanji \& Beer, 2012; Frankort et al., 2012; Grabenhorst et al., 2008; Hare et al., 2011; Hege et al., 2018; Pohl et al., 2017; Schroder et al., 2014; Siep et al., 2012; Yokum \& Stice, 2013). In these studies, it was for example found that the focus of attention - in interaction with weight status - influenced neural responses to visual food stimuli (Frankort et al., 2012; Hare et al., 2011; Siep et al., 2012; Yokum \& Stice, 2013). More specifically, it has been shown that neural responses in the mesocorticolimbic system were greater in overweight people than in healthy-weight people when participants were required to focus on the tastiness of stimuli (hedonic focus), but not when they were not given any instructions on how to evaluate the presented foods (Frankort et al., 2012). Moreover, 
neural activity has been shown to be decreased by suppressing food craving relatedthoughts while viewing high caloric food stimuli (Siep et al., 2012). This suggest that neural responses can be modulated by cognitive strategies. Neural responses to the actual experience of taste and of pleasure can be influenced by peoples' mindset or beliefs as well. It was for example shown that neural activity in the OFC was influenced while tasting wine by manipulating price labels. More specifically, presenting the same wine stimuli with different price-labels showed that higher priced wines were rated as more pleasurable and led to an increased activity in the OFC, as compared to lower priced wines (Plassmann et al., 2008). Similarly, taste ratings and neural responding in the medial OFC, amygdala and striatum were also affected by different labels on cola (River Cola, T Cola, Coca Cola or Pepsi Cola)(Kühn \& Gallinat, 2013). Here, increased activity in right mOFC during weak (River Cola and T Cola) compared to strong brand (Coca Cola and Pepsi Cola) cues was found and stronger activation in the right amygdala was found for Coca Cola cues as compared with Pepsi Cola cues. Furthermore, during the taste phase the same beverage elicited stronger left ventral striatum activation when it was coupled with a strong as compared to a weak brand. These studies indicate that neural responding to food stimuli can be significantly modulated by expectations, the focus of attention or task demands, and illustrate that it is important to have a well-controlled task design to allow for a better interpretation of the observed neural activity.

An additional concern is that the precise role of the dopaminergic mesocorticolimbic system is ambiguous, as it is involved in several mental processes, such as processing something with a highly positive value or a highly negative value (Chikazoe et al., 2014; Kahnt et al., 2014). But in the context of examining neural food processing, it is often assumed that neural activity in the mesocorticolimbic system in response to (high caloric) food stimuli is proportionate to the foods' rewarding value. However, this is certainly not that straightforward. Instead, the dopaminergic mesocorticolimbic system was also considered an overarching motivational saliency system (Salamone \& Correa, 2012). This means that an increased neural activity in the mesocorticolimbic system is observed for both highly positive and highly negative stimuli as compared to neutral stimuli, when they are equally salient. In line with this idea, several fMRI studies observed a similar level of activity in the mesocorticolimbic system to both rewarding and aversive stimuli (Chikazoe et al., 2014; Kahnt et al., 2014; Pfabigan et al., 2014; Pujol et al., 2018; Suzuki et al., 2017). These findings suggest that neural activity in brain regions of the mesocorticolimbic system reflect motivational saliency (either negative or positive) rather than reward value. 
These finding also suggests that the level of activation does not always provide enough information. Mass-univariate analysis of fMRI data is only informative regarding the involvement of brain areas in certain tasks, whereas multivoxel pattern analysis of fMRI data (MVPA) can decode representational content in the brain (Norman et al., 2006). With MVPA, the multi-voxel pattern of activity in a certain brain region is determined, with all individual included voxels - with varying levels of activity - contributing to information representation, whereas in univariate analyses only the level of activity is considered, averaged across voxels in a brain region (see Figure 2). Interestingly, a recent study showed that food value can be decoded above chance from multi-voxel patterns of neural activity in both the lateral and medial OFC (Suzuki et al., 2017). Another study showed that positive versus negative values could only be differentiated by MVPA, but not by mass-univariate analyses (Chikazoe et al., 2014).

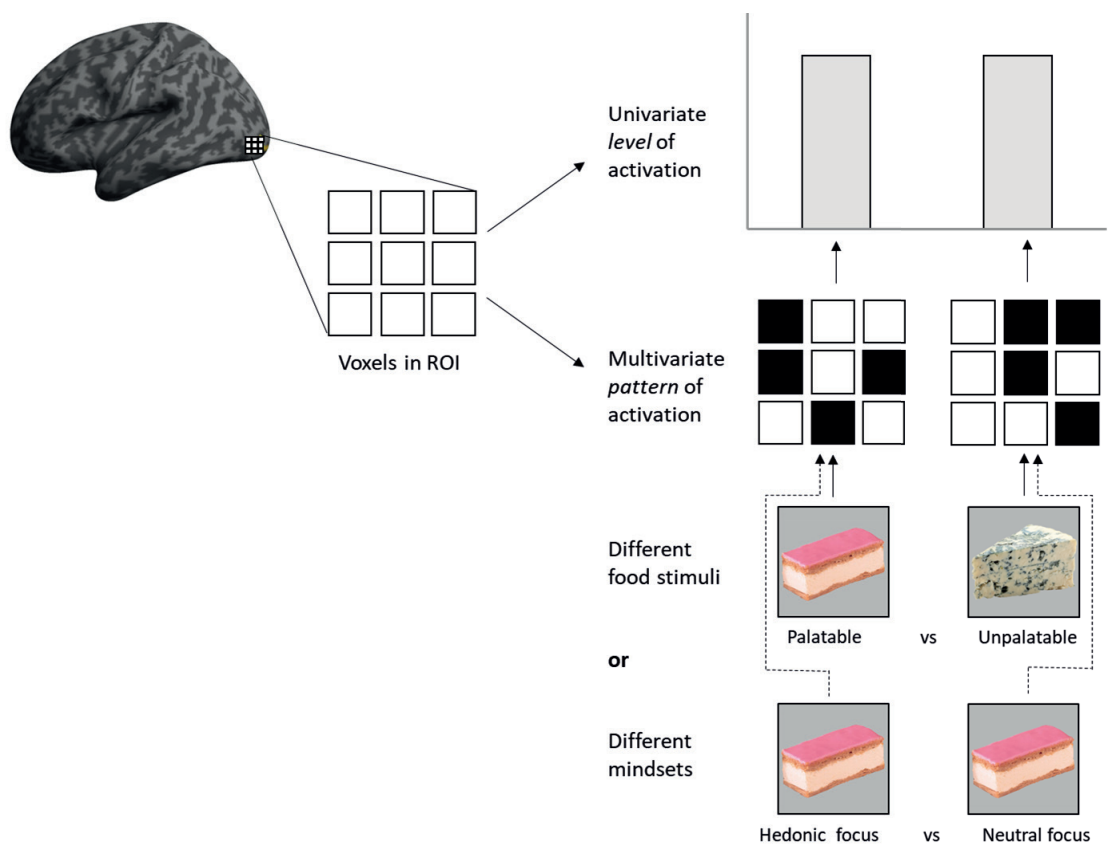

Figure 2. Hypothetical neural responses to food stimuli as measured with functional magnetic resonance imaging (fMRI) and by using a univariate and multivariate analysis approach. The food stimuli given would be individually tailored on palatability. These nine hypothetical voxels in a region of interest $(\mathrm{ROI})$ can have the same level of activation, whereas the multi-voxel pattern of activation can be very different. Here, the effects of different food stimuli (e.g. viewing palatable versus unpalatable food stimuli) and different mindsets (e.g. hedonic versus neutral focus to food stimuli) are illustrated. Picture is inspired by Fig. 1 of Mur et al., (2009). 


\section{Gut-hormonal responses}

Gut hormones interact with brain responses to regulate appetite. For this reason, targeting gut hormones like GLP-1 and ghrelin responses to reduce appetite is thought to be a possible solution for overeating (Murphy \& Bloom, 2006). However, using gut hormones as therapeutic target seems not that simple or effective for weight loss, and this type of intervention is associated with side effects (e.g., nausea) (Chaudhri et al., 2008).

There is also some evidence that mindset influences peripheral metabolic hormonal processes involved in appetitive motivation. For example, previous studies showed that mindset influences the ghrelin and GLP-1 responses, which in turn activates the regulation of food intake (Cassady, Considine, \& Mattes, 2012; Crum, Corbin, Brownell, \& Salovey, 2011). Crum and colleagues (2011) showed that expectations of the caloric content affected the ghrelin response. More specifically, the ghrelin level rose from baseline to viewing the label (just before consumption) and dropped after consumption, only when the drink was presented as a high-caloric indulgent drink. The ghrelin-response was flat when the drink was presented as a sensible healthy drink. Another study illustrated that GLP-1 secretion was affected by beliefs about the satiating effects of a beverage, while the nutritional content of the beverages was kept exactly the same. Here, participants consumed oral liquid and solid preloads and were informed of their postingestive properties through a demonstration to believe that the preloads were either liquid or solid (more satiating) in their stomach. It was found that GLP-1 secretion increased or decreased when participants believed to be drinking a more or less satiating beverage (Cassady et al., 2012). These studies show that mindsets or cognitive modulations interact with hormonal processes involved in appetitive motivation.

\section{Metabolic response}

Research has shown that there is a positive relationship between the energetic caloric load of food and the metabolic response after consumption. A systematic review indicated that more energy intake (as expressed in kJ) led to a higher TEF (Quatela et al., 2016). In addition, next to energy content also nutritional composition can alter TEF. When participants receive three isocaloric meals on separate days, the meal rich in proteins led to the highest TEF as compared to meals high in fat or carbohydrate. Also subjective satiety rating are higher after eating a high protein meal (Crovetti et 
al., 1998). These studies suggest that TEF can be influenced by energy caloric load and by nutritional composition. In addition, it shows the relationship between TEF and satiety feelings.

To the best of our knowledge, there is no evidence yet that a mindset can influence metabolic responses involved in eating behaviour (e.g., TEF). What has been found was that the actual energy content and sweet taste influenced the TEF. Research has shown a positive relationship between caloric load and the metabolic response after consumption (Crovetti et al., 1998). A recent systematic review indicated that in humans, more energy intake led to a higher metabolic response, also called dietinduced thermogenesis (Quatela et al., 2016). Thus far, research suggested that it seems that sweetness and energy have their own oral and post-oral receptors, leading to specific metabolic responses (Burke \& Small, 2015; Chambers et al., 2009; Veldhuizen et al., 2017). That is, sweetness and calories are independently positively correlated with an increased metabolism. However, as hormones can also be influenced by mindset manipulations it could be that manipulating expectations on caloric content, metabolic responses might be influenced as well. When expecting to consume a high caloric preload, this perception might could increase the TEF.

\section{Food cue exposure therapy: a solution for overeating?}

As described earlier, one of the main factors contributing to overeating is an increased food-cue reactivity. These food cues can be internal, such as hunger, but also external, such as the smell, sight and taste of food, or environmental contexts (Boswell \& Kober, 2016; Jansen et al., 2016). During food cue exposure therapy, people are repeatedly exposed to food cues (e.g., the sight and smell of food) while eating the foods is not allowed (Jansen et al., 2011, 2016; van den Akker, Havermans, et al., 2014). After repeated non-reinforced exposure sessions, the food cue reactivity decreases (Jansen et al., 2011, 2016). The assumed mechanism behind food cue exposure therapy is that a new association between the food cue and intake is formed, namely that the food cue does not predict intake. Importantly, the old association is not unlearned (Bouton, 2004, 2011; Jansen et al., 2016); instead, a new inhibitory association is learned. Due to this inhibitory learning, reactivity to food cues diminishes. 


\section{This dissertation}

Taken together, several studies suggested that mindset (e.g. attentional focus, and/ or other cognitive modulations) can alter physiological and psychological factors involved in food processing. However, there is still much inconsistency in the existing literature on (food) brain reward processing in general, food brain reward processing in obesity, and there is limited evidence on the influence of a mindset or other cognitive modulation on hormonal responses and metabolism. The overarching aim of this dissertation is to examine the influence of mindset on psychological and physiological variables involved in eating behavior. The central hypothesis of this dissertation is that a hedonic mindset elicits neural, hormonal, metabolic and behavioral responding reflective of reward value, whereas a health or neutral mindset elicits neural, hormonal, metabolic and behavioral responding reflective of control and conflict.

One of the aims is to examine the role of an attentional focus on neural (reward) processing to food stimuli and the ambiguous function of the mesocorticolimbic system herein in females who are overweight. In a within-subjects design, we will examine neural responses to individually tailored palatable and unpalatable high caloric food stimuli, while participants' attentional focus is manipulated to be either hedonic or neutral (chapter 2). We will analyze the fMRI data with a univariate and multivariate approach. Here, two hypotheses will be pitted against each other. First, a greater neural (reward) response to palatable than to unpalatable food stimuli is expected, and even more pronounced with a hedonic focus. On the other hand, if the neural responses to food stimuli should be viewed as reflecting motivational saliency, only a main effect of attentional focus (hedonic vs neutral) is expected. Furthermore, we expect that multivariate analyses is able to distinguish between neural representations of palatable versus unpalatable foods, and most clearly for the hedonic focus blocks. The second study (chapter 3) examines the effects of a more general control or loss of control mindset on behavioural, neural and hormonal responses (ghrelin and GLP-1) towards chocolate. In a within-subjects design, a loss of control and a control mindset will be induced by having female chocolate lovers view a short movie. Subsequently, neural responses to visual chocolate stimuli will be measured using fMRI in an ultra-high field scanner (7T). Hormone levels will be assessed on five pre and post mindset induction moments and they will be simultaneously assessed with self-reported feelings of chocolate craving, hunger 
and control. Furthermore, chocolate intake will be measured after the mindset manipulation in a bogus chocolate taste test. It is hypothesized that the manipulated loss of control mindset will lead to hormonal and neural responses that prepare for ongoing food intake, even after eating, while the manipulated control mindset will lead to hormonal and neural responses reflecting satiety. More specifically, a loss of control mindset - as compared to the control mindset - is expected to lead to increased self-reported cravings and hunger, decreased feelings of control, increased chocolate consumption, increased ghrelin levels, and decreased GLP-1 levels. For the level of neural activity (mass-univariate analyses), we expect increased activity in reward-related brain areas to chocolate versus neutral images in the loss of control mindset, whereas, more control-related activity is expected to chocolate stimuli in the control mindset. For multivariate analyses, differences are expected between the two mindsets in distinguishing neural representations of chocolate versus neutral images and predominantly in food-reward brain areas when in a loss of control mindset. In chapter 4, the aim is to examine the influence of caloric expectations induced by labels on metabolic response after consuming a sweetened beverage. The TEF will be calculated after consuming three mid-caloric (112.5 kcal) and equally sweetened beverages with different caloric content labels (low-, mid- and high-caloric). The goal is to elucidate if metabolism could be activated purely based on labels and not by actual caloric content. It is expected that that TEF will be higher with a high-caloric label than with a low-caloric label. A final objective is to study the effect a food cue exposure therapy as compared to a control lifestyle intervention on neural responding to visual food cues after. Therefore, in chapter 5, we aim to find neural correlates of a successful food cue exposure therapy. Here, ten women will be examined to measure the response to individually tailored visually presented palatable high-caloric food stimuli before versus after a cue exposure intervention $(n=5)$ or a control lifestyle $(n=$ 5) intervention. Data will be analyzed case-by-case. It is hypothesized that participants after food cue exposure therapy will show reduced neural activity in brain regions related to food cue reactivity (i.e. ventral striatum with nucleus accumbens (NAcc), midbrain, OFC, anterior insula (INS), gustatory cortex (GC), lateral occipital cortex (LOC), and somatosensory cortex (SSC)) and increased neural activity in brain regions related to inhibitory-control (dorso- and ventrolateral prefrontal cortex (dIPFC, vIPFC), parietal posterior cortex (PPC), dorsal anterior cingulate cortex (ACC), caudate, pre supplementary motor area (preSMA) and the globus pallidus (GP)) as compared to participants receiving a control-lifestyle intervention. In chapter 6 , the main results 
of the studies of this dissertation are summarized and discussed, and directions for future research are provided. 


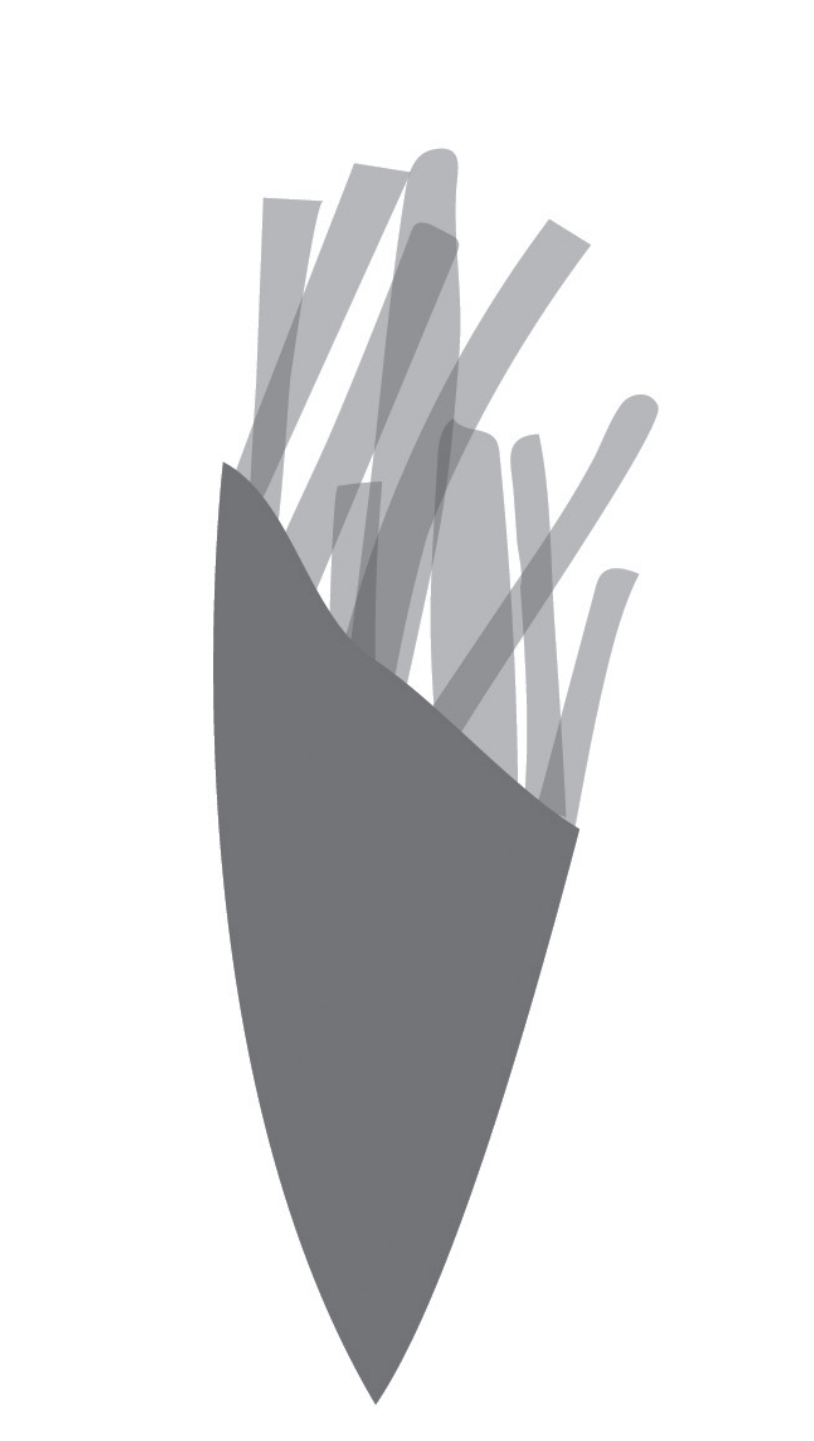




\section{CHAPTER 2}

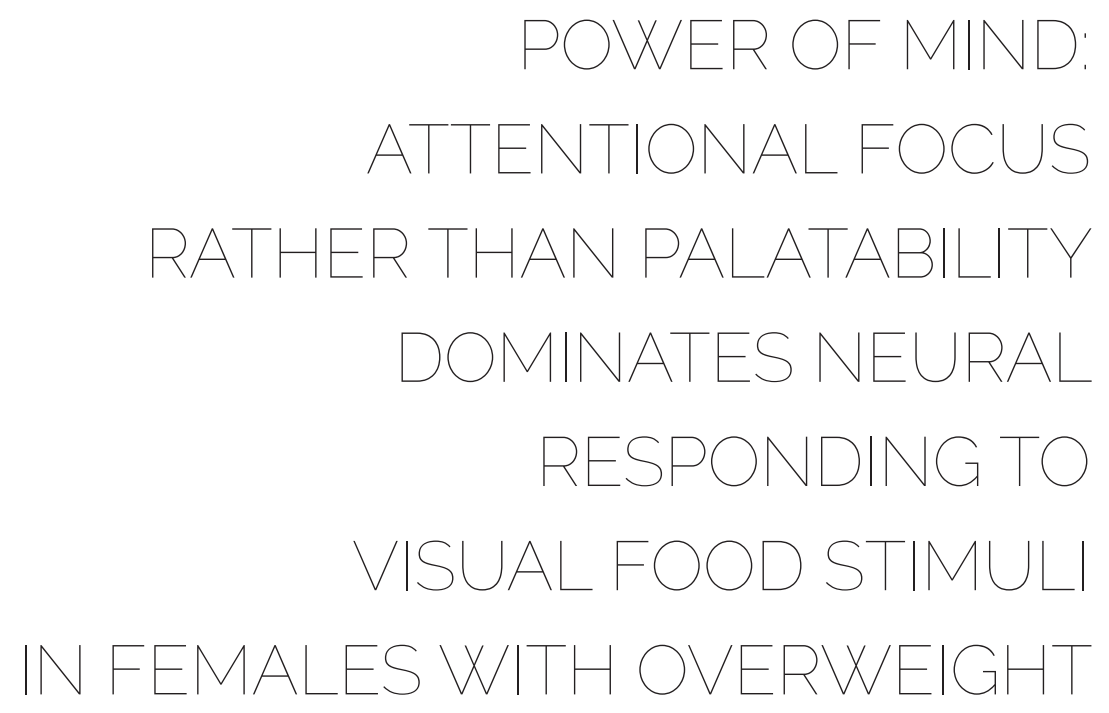

Published as: Franssen, S., Jansen, A., van den Hurk, J., Roebroeck, A., and Roefs, A., (2020). Power of mind: Attentional focus rather than palatability dominates neural responding to visual food stimuli in females with overweight. Appetite, 148, 104609. https://doi.org/10.1016/j.appet.2020.104609 


\section{Abstract}

Research investigating neural responses to visual food stimuli has produced inconsistent results. Crucially, high-caloric palatable foods have a double-sided nature - they are often craved but are also considered unhealthy - which may have contributed to the inconsistency in the literature. Taking this double-sided nature into account in the current study, neural responses to individually tailored palatable and unpalatable high caloric food stimuli were measured, while participants' (females with overweight: $n=23$ ) attentional focus was manipulated to be either hedonic or neutral. Notably, results showed that the level of neural activity was not significantly different for palatable than for unpalatable food stimuli. Instead, independent of food palatability, several brain regions (including regions in the mesocorticolimbic system) responded more strongly when attentional focus was hedonic than when neutral $(p<0.05$, FWE corrected). Multivariate analyses showed that food palatability could be decoded from multi-voxel patterns of neural activity $(p<0.05$, FDR corrected), mostly with a hedonic attentional focus. These findings illustrate that the level of neural activity might not be proportionate to the palatability of foods, but that food palatability can be decoded from multi-voxel patterns of neural activity. Moreover, they underline the importance of considering attentional focus when measuring food-related neural responses.

\section{Keywords}

fMRI, food-reward, multi-voxel pattern analysis, obesity 


\section{Introduction}

Since 1975, the number of adults with obesity (BMI $\geq 30 \mathrm{~kg} / \mathrm{m}^{2}$ ) has nearly tripled worldwide (World Health Organization, 2017). The population with obesity is at high risk of health complications and is often unsuccessful at dieting and maintaining weight-loss (Wing \& Phelan, 2005). Therefore, examining mechanisms involved in the maintenance of obesity is of major importance. Obesity is ultimately caused by a prolonged energy imbalance: the number of calories consumed exceeds the number of calories expended (Hall et al., 2012; Mitchell et al., 2011). Our current obesogenic environment is an often-cited cause for this energy imbalance (Hill et al., 2000). However, we all live in the same environment, but not everyone is obese. It has therefore been proposed that people who are overweight and obese may be more sensitive to this environment. Indeed, research has found that they show increased high-caloric food-cue reactivity (Boswell \& Kober, 2016). Food-cue reactivity is the appetitive responding, like craving and salivation, to cues predicting food intake (Jansen, 1998; Jansen et al., 2015). In its turn, this food-cue reactivity makes it more difficult to refrain from consuming those foods (Boswell \& Kober, 2016; Jansen et al., 2015).

Theoretically, food-cue reactivity is thought to be reflected as neural activity in the dopaminergic mesocorticolimbic system (Leig \& Norris, 2018; Volkow et al., 2013; Volkow et al., 2011). Indeed, several studies have observed neural activity in brain regions belonging to this system, but observed neural activity has not been restricted to this system (Frankort et al., 2014; Ng et al., 2011; Scharmüller et al., 2012; Yokum et al., 2011). Areas that have been associated with food reward processing are amygdala, hippocampus, ventral pallidum, nucleus accumbens and striatum, as well as the anterior cingulate, orbitofrontal cortex (OFC), insular, posterior fusiform, (ventro)medial and dorsolateral prefrontal cortices (vmPFC and dIPFC) (Berthoud et al., 2011; Haber \& Knutson, 2010; van der Laan et al., 2011). Note that the anterior cingulate, dorsolateral, and medial prefrontal cortices are also considered to have a regulatory function in neural food-cue reactivity (Giuliani et al., 2018). Of concern is that the evidence that these brain regions are involved in processing visual food stimuli is quite inconsistent. A meta-analysis (van der Laan et al., 2011) found that the concurrence of activated clusters in visual food processing (food versus non-food) between studies in healthy weight individuals was moderate. Concurrence of activated clusters was shown in the bilateral posterior fusiform gyrus, lateral occipital complex 
(LOC), the left lateral OFC and the left middle insula. Importantly, food palatability was not taken into account in the studies included in this meta-analysis, as the contrast of interest was food versus non-food in most studies. Another meta-analysis showed that the computation of food palatability (i.e. subjective value) was primarily neurally represented in the vmPFC (Clithero \& Rangel, 2014).

It is theorized that neural anticipatory reward is increased in people with overweight (Leigh \& Morris, 2018; Stice et al., 2013). Indeed, some functional magnetic resonance imaging (fMRI) studies showed that participants who are overweight or obese have increased activity in one or more of these brain areas when viewing food stimuli compared to lean controls (Frankort et al., 2012; Pursey et al., 2014; Rothemund et al., 2007; Stoeckel et al., 2008; Yokum et al., 2011), and some studies found this increased activity to be more pronounced for high-caloric than for lowcaloric foods (Rothemund et al., 2007; Stoeckel et al., 2008). However, other studies showed either reduced or no significant differences in activity (Frankort et al., 2012; Stice et al., 2010) in people with overweight versus healthy-weight (Brooks et al., 2011; Schienle et al., 2009). So, the picture of brain food-reward processing in obesity is far from clear. This inconsistent pattern of results became quite apparent in a recent review on this topic (Ziauddeen et al., 2012) in which it is stated that both the brainregions in which effects were observed and the direction of effects varied highly over studies, which led the authors to conclude that "... the pattern emerging from studies comparing obese individuals and binge-eaters with controls is most remarkable for its variability and inconsistency "(p.283). Likewise, a very recent review focusing on the convergence of the role of dopamine between animal and human studies also emphasizes the inconsistency of evidence for the dopaminergic system involvement in the neurocognitive profile of obesity (Janssen et al., 2019). So, the idea of increased anticipatory neural responding in people with overweight as compared to healthyweight is not backed up by solid consistent empirical evidence, and more research is needed.

Relevant to this discussion is that previous studies often presented visual food stimuli in a so-called passive viewing paradigm (Martin et al., 2010; Rothemund et al., 2007; Stoeckel et al., 2008; Thomas et al., 2015). In this paradigm, participants are only instructed to look at the presented stimuli, without performing any stimulusrelated task, assuming that just viewing the visual food stimuli will trigger a rewardresponse. However, the perception of high-caloric food stimuli has a double-sided nature: high-caloric foods often have a high hedonic value and simultaneously a low 
health value because their overconsumption contributes to weight gain (Roefs et al., 2018). Research using passive viewing paradigms assumes that people automatically focus on the palatability of high-caloric foods (Martin et al., 2010; Rothemund et al., 2007; Stoeckel et al., 2008; Thomas et al., 2015). However, people may actually not (only) focus on the palatability of food, but (also) on the health-value of food, and they may be alternating between those frequently (Roefs et al., 2018). Importantly, this may happen - unbeknownst to the researcher - within and across participants as well as within and across studies, complicating the interpretation of this type of brain imaging studies. Crucially, the exact ongoing mental process while people perform a passive viewing paradigm is unknown (Roefs et al., 2018). So, conclusions in this literature seem to depend mostly on reverse inference: "to infer the likelihood of a particular mental process $M$ (i.e., reward responsiveness) from a pattern of brain activity $A$ (i.e., activity in the mesocorticolimbic system)" (Poldrack, 2011) (P. 693). People who are overweight - who often have a dieting history and score relatively high on measures of dietary restraint (Herman \& Polivy, 1980) - may be especially likely to alternate their focus of attention between evaluating palatability and health value of high caloric foods. To be able to adequately interpret an observed pattern of neural activity, it is necessary to be certain about the ongoing mental process.-

Consistent with this line of reasoning, it has been shown that expectations strongly influence neural responding while the sensory input for participants is the same (Yeshurun et al., 2017). Crucially, this has also been observed for food perception (Frankort et al., 2012; Grabenhorst et al., 2008; Hare et al., 2011; Pohl et al., 2017; Siep et al., 2012; Yokum \& Stice, 2013). That is, the focus of attention - in interaction with weight status - influences neural responses to food stimuli (Frankort et al., 2012; Hare et al., 2011; Siep et al., 2012; Yokum \& Stice, 2013). These previous findings suggest that neural responding to food stimuli can be significantly modulated by attentional focus and task demands, and indicate that it is important to have a well-controlled task design with a well-defined mental process, to allow for a better interpretation of the observed neural activity.

An additional concern is that the precise function of the dopaminergic mesocorticolimbic system remains elusive. Many researchers consider the embedded brain areas mainly as a system processing reward (see for a review Volkow, Wang, \& Baler, 2011). However, other researchers consider it a more general overarching motivational saliency system (Salamone \& Correa, 2012), because the level of activity to both rewarding and aversive stimuli is similar, as observed in quite some fMRI 
studies (Chikazoe et al., 2014; Kahnt et al., 2014; Pfabigan et al., 2014; Pujol et al., 2018; Suzuki et al., 2017). For example, the OFC was found to respond similarly to both rewarding and aversive stimuli (Chikazoe et al., 2014; Kahnt et al., 2014; Suzuki et al., 2017), and is also involved in motivational saliency (Rothkirch et al., 2012). This seems in contradiction to the meta-analysis of Clithero and Rangel (2014) which might be due to a lack of inclusion of clearly negative stimuli in the studies included in the meta-analysis.

An interesting possibility is that the distinction between positive and negative value can be made with multivariate data analysis, that is, considering multi-voxel patterns of neural activity. Whereas univariate analyses of fMRI data are informative regarding involvement of brain areas in certain tasks, multivariate analysis of fMRI data can decode representational content in the brain (Norman et al., 2006). With multivariate analyses of fMRI-data, one looks at the multi-voxel pattern of activity in a certain brain region, with all individual included voxels - with varying levels of activity - contributing to information representation, whereas in univariate analyses one only considers the level of activity, averaged across voxels in a brain region. In line with this idea, a recent study showed that food value can be decoded from multi-voxel patterns of neural activity in both lateral and medial OFC (Suzuki et al., 2017). Additionally, another study (Chikazoe et al., 2014) showed that a multivariate analysis was able to differentiate between positive versus negative values, whereas a univariate approach was not. That is, in univariate analyses, the neural responses in the medial OFC and vmPFC to positive and negative value largely overlapped. Importantly, positive versus negative visual and taste stimuli could be decoded from multi-voxel patterns of neural activity in the lateral and medial OFC. These findings illustrate that a distinction between positive and negative value could only be found if a multivariate analysis approach to data analysis was used.

Therefore, the present study aimed to elucidate neural representations of food and how attentional focus affects these neural representations. Crucially, our study builds on previous research by: (1) using a paradigm with a clear stimulusevaluation-task to overcome the disadvantages of passive viewing paradigms and avoid the trap of reverse inference, (2) including individually tailored highly palatable and highly unpalatable high-caloric food stimuli, and (3) implementing both univariate and multivariate fMRI-analyses. While in the scanner, females with overweight were required to apply one of two attentional foci during a fast-paced 1-back task: a hedonic focus or a neutral focus while being presented with palatable and unpalatable high- 
caloric food stimuli. Two main hypotheses were pitted against one another. On the one hand, in line with the view that the level of neural activity reflects food reward value, a greater neural response to palatable than to unpalatable food stimuli was expected, and even more pronounced with a hedonic focus. If, on the other hand, the neural responses to food stimuli should be viewed as reflecting motivational saliency, only a main effect of attentional focus (hedonic vs neutral) should be observed. We furthermore expect, in line with findings of Chikazoe et al., (2014) and Suzuki et al., (2017), that multivariate analyses (i.e., multi-voxel pattern analysis; MVPA) can distinguish between neural representations of palatable versus unpalatable foods, and most clearly for the hedonic focus blocks.

\section{Materials and Methods}

\section{Participants}

Twenty-four females with overweight (BMI $\geq 27 \mathrm{~kg} / \mathrm{m}^{2}$; age 18 - 60) from the Maastricht area were recruited. ${ }^{1}$ An initial threshold for overweight was set at BMI of 27 , to be relatively sure that all participants would be overweight upon objective measurement $(\mathrm{BMI}>25)$. Data from one participant was excluded due to excessive movement during the scan-session. This resulted in a dataset of 23 participants (age: $M=39.5$, $S D=11.0$ years, BMl: $M=33.2, S D=4.7$, range $26.64-45.91$ ). All participants, except for two, were right-handed. All participants were screened to exclude those who had contra-indications for scanning. Furthermore, we screened for any current cardiac arrhythmia, circulatory problems, diabetes, epilepsy, and psychological treatment, and for past surgery on head or brain. The study was approved by the Ethical Committee of the Faculty of Psychology and Neuroscience of Maastricht University, The Netherlands. The participants gave their written informed consent and received gift vouchers ( $€ 20$ ) as compensation. The sample size was based on previous studies in the same research domain using similar analysis approaches (Chikazoe et al., 2014; Suzuki et al., 2017; Yokum \& Stice, 2013) and it is considered sufficient for a withinsubjects blocked-design, achieving at least $80 \%$ power with a family-wise alpha of 0.05 (Cremers et al., 2017; Desmond \& Glover, 2002; Mumford \& Nichols, 2008).

\footnotetext{
${ }^{1}$ Of this group, data of 10 participants were also included as a pre-measurement in a study testing the pre-post effect of cue exposure therapy vs. control therapy on neural responses to visual food stimuli. In these analyses, pre- and post-therapy neural responding was analyzed as case-series (manuscript submitted for publication).
} 


\section{Behavioral assessments}

\section{Hunger assessment}

To limit the influence of hunger, participants were asked to refrain from eating or drinking, besides water, for at least one hour before the start of the experiment. To check compliance, experienced hunger was measured before the start of the experiment on a $100 \mathrm{~mm}$ visual analogue scale (VAS), with the question: "How hungry do you feel at this moment?" ranging from 0 (not hungry at all) to 100 (very hungry). Furthermore, participants registered when they had eaten their last meal and what they had consumed at that time.

\section{Individual stimulus selection and liking rating.}

Individual food stimulus selection was completed approximately a week before the scanning session with an online questionnaire. Each participant was asked to choose and rate her 5 most palatable foods and 5 most unpalatable foods from a list of 33 high caloric foods. Ratings of the selected stimuli were provided on 10-point rating scales ranging from 1 (not palatable at all) to 10 (very palatable). See supplementary Figure 1. for example stimuli.

\section{Stimuli}

For each selected food item, two different pictures (varied across blocks, not within a block) were included in the fMRI stimulation protocol for that participant, to avoid presenting exactly the same stimulus too often. Neutral stimuli were plants and flowers, and were the same for all participants, with again 2 pictures per item. In total, each participant viewed 30 different pictures during the fMRI task. Pictures were presented as high-resolution coloured images as pop-out figures on a light grey background (RGB: 191191 191; CKYM: 252020 0) in the centre of a black screen, covering a visual angle of approximately $12^{\circ}$.

\section{Experimental task}

\section{fMRI Stimulation protocol}

The fMRI task consisted of four functional runs. Each functional run contained 
each of six block types with all combinations of stimulus type and attentional focus represented: (1) high caloric palatable foods - attentional focus neutral, (2) high caloric palatable foods - attentional focus hedonic, (3) high caloric unpalatable foods - attentional focus neutral, (4) high caloric unpalatable foods - attentional focus hedonic, (5) neutral control stimuli - attentional focus esthetics and (6) neutral control stimuli - attentional focus neutral. In each block, each of 5 stimuli were presented twice (so 10 in total). Block-order was randomized within each of these four runs, and this randomized order was then mirrored within the run, to have each block presented twice in each run (e.g., 1-4-2-6-3-5-5-3-6-2-4-1) for a total of 12 blocks per run. Run-order was randomized across participants. The anatomical scan was acquired between functional runs 2 and 3 .

Prior to each block, an attentional focus cue-word "tasty", "beauty" or "color" was presented for 1 second. Blocks lasted 20 seconds and were always followed by a 20 second rest block (fixation cross), resulting in a run length of $513 \mathrm{~s}$, and total functional scanning time of 35 minutes. The stimuli were presented using E-prime (version 2.0, Psychology Software Tools Inc., Pittsburg, PA, USA). ${ }^{2}$

\section{Attentional focus manipulation}

To induce the attentional focus, participants performed a fast-paced 1-back task in each functional run, with the exact instruction depending on the blocktype. Each stimulus was presented for 500 ms, with an inter-stimulus interval (ISI) response window of 1500 ms. During the ISI, a fixation cross, a red minus sign (left), and a and green plus sign (right) were presented indicating what button to press. The participant's responses were registered using a button box, with a right index finger press for "less" or "fewer" (indicated by red minus sign) and a right middle finger press for "more" (indicate by green plus sign). The participant was asked to compare each presented picture (starting from the $2^{\text {nd }}$ picture of each block) to the previously presented picture within a block, and to indicate whether the presented food was either more or less palatable (hedonic focus), or contained more or fewer colors (neutral focus) than the previous one. As the neutral stimuli cannot be evaluated on its palatability, we chose for a comparable subjective evaluation and asked if the

\footnotetext{
${ }^{2}$ Notes: (1) Due to a programming error in E-prime, the first 12 participants received 10 blocks with 12 stimuli each (instead of 12 blocks with 10 stimuli each), which resulted in a block duration of $24 \mathrm{~s}$. We accounted for this error in our analysis and no data had to be excluded for this reason, as in total (after four runs with randomized block-order) enough data per condition was collected. (2) For one participant, only data of 3 runs was included due to set up problems.
} 
picture was more or less beautiful (esthetic focus) than the previous one to keep the design balanced. The neural focus condition was the same for the neutral stimuli as for the food stimuli.

\section{Statistics: Difficulty level variants 1-back task}

To check task difficulty of the different variants of the 1-back task, response latencies were registered. Response latency increases with rising difficulty of a perceptual task (Schneider et al., 2011). All trials with a missing button press (1.42 \%) and trials with a response latency of 2.5 SD below (response too fast: < $89.26 \mathrm{~ms}$ ) or above (response too slow: > $1481.37 \mathrm{~ms}$ ) the mean latency (2.75 \%) across all participants were excluded from analyses. Average response latencies were analysed in a 2 (food type: palatable vs unpalatable) * 2 (attentional focus: neutral vs hedonic) ANOVA because our main fMRI analyses focused on food types with the two attentional foci. For our additional fMRI analysis, in which we compared each food type - attentional focus condition to non-food neutral stimuli - neutral focus condition, average response latencies per relevant condition were compared using paired samples $t$-tests. We also tested the effect of attentional focus for the neutral stimuli, by comparing the average response latencies for neutral focus to the aesthetic focus using paired samples t-tests.

\section{Statistics: visual features stimuli}

To assess visual features of our stimuli, participants did an offline stimulus similarity task. Here, all stimuli used in the individually tailored fMRI task were randomly presented in pairs of two, and participants were asked to evaluate the similarity in color and shape of each pair on a 5-point rating scale (1: not similar at all to 5: highly similar). Stimulus categories were paired in 6 ways: unpalatable - unpalatable food, unpalatable - palatable foods, unpalatable food - neutral, palatable - palatable food, palatable food - neutral and neutral - neutral. Each participant rated all possible pairs of 30 stimuli first on color and then on shape, leading to 105 pairings per rating-type. We wanted to make sure that our stimulus categories were not (partly) defined by perceptual features, which would be the case if the within-category perceptual similarity was larger than the between-category similarity. We therefore tested whether within-category similarity (e.g., palatable food - palatable food) was larger than between-category similarity. (e.g., palatable food - unpalatable food). Specifically, we compared the average shape and color within-category similarities 
to the average shape and color between-category similarities using paired-samples t-tests (see Peelen \& Caramazza (2012) for a similar approach). E-prime (version 2.0, Psychology Software Tools Inc., Pittsburg, PA, USA) was used to present the stimuli and record the ratings.

\section{Experimental procedure}

All scanning sessions took place during the day between 9:00 AM and 5:00 PM. After providing informed consent, the participant filled in the hunger assessment. Next, she received experimental instructions and did an offline practice of the fMRI-task (1 run, different stimuli), after which the one-hour scanning session started. Subsequently, the participant was asked to compare the stimuli on color and shape (offline). At the end of the experiment, height and weight were measured to compute BMI ( $\left.\mathrm{kg} / \mathrm{m}^{-2}\right)$, and the participant received a gift voucher, and was debriefed.

\section{MRI data acquisition}

Imaging was performed on a 3 Tesla MRI scanner (Magnetom Prisma Fit, Siemens Medical Systems) with a 64 channel head/neck coil located at Scannexus Maastricht, The Netherlands. Each participant was placed comfortably in the scanner head coil with her head fixated using foam pads. Via an attached mirror to the head coil could the participant see the projected stimuli on a screen at the end of the scanner bore. Functional ( $\mathrm{T}_{2}{ }^{*}$-weighted) images were acquired in an axial interleaved order using multiband gradient echo-planar imaging (Feinberg et al., 2010) $(\mathrm{TR}=2000 \mathrm{~ms}$, TE $=30 \mathrm{~ms}$, flip angle $=77^{\circ}, \mathrm{FOV}=208 \times 124 \mathrm{~mm}^{2}$, voxel size of $2 \times 2 \times 2 \mathrm{~mm}, 62$ slices with multiband factor 2 and GRAPPA2). Slices were acquired in a backward tilted direction of approximately 15 degrees to the transversal - coronal line and covered the whole brain. Each participant underwent four experimental functional runs, with approximately 260 volumes acquired per run. Also a high-resolution three-dimensional (3D) $T_{1}$-weighted anatomical scan was acquired (MPRAGE pulse sequence, $\mathrm{TR}=2250 \mathrm{~ms}, \mathrm{TE}=2.21 \mathrm{~ms}$, flip angle $=9^{\circ}$, FOV $=256 \times 192 \mathrm{~mm}^{2}$, voxel size $1 \times 1 \times 1 \mathrm{~mm})$.

\section{Preprocessing fMRI data}

Analyses were performed using SPM12 (Statistical Parametric Mapping, London, UK) and Matlab version 8.3.0.532 (R2014a). First, functional images were slice-time corrected by realigning the acquired voxel time series to the middle slice. Pre- 
processing included three-dimensional (3D) motion correction (using second degree B-spline interpolation) to compensate for small head movements by spatially aligning the volumes of each functional run to the mean. The estimated translation ( $x, y$ and $z$ ) and rotation parameters (roll, pitch and yaw) that resulted from the motion correction were inspected and used as a criterion for excessive movement. The functional data were excluded from statistical analysis when movement exceeded three millimeters or degrees in any direction within a run. For co-registration of each participant's data, the anatomical scan was warped to mean functional data space. Thereafter, images were spatially normalized and temporally filtered using a high-pass filter with a cutoff period of $128 \mathrm{~s}$. For the normalization process, unified brain segmentation was performed prior to transformation of the images to MNI space (Montreal Neurological Institute, Montreal, Canada). Deformation fields derived from segmentation were used to transform all the functional images to MNI space. Finally, spatial smoothing was applied to the data using a Gaussian kernel of $6 \mathrm{~mm}$ full width at half-maximum (FWHM). Pre-processed and normalized functional volume time series were then used for the statistical analysis.

\section{Univariate fMRI analysis}

On the first level of analysis, for each participant, a general linear model (GLM) design matrix was created including the four runs as separate sessions. Each experimental task condition was set as a predictor, which resulted in six predictors of interest per run. Predictor time courses were obtained using condition box-car shaped waves convolved with a two-gamma ideal hemodynamic response function (HRF). Additionally, six motion and four run mean intensity predictors of no interest were added to the model as nuisance regressors. To investigate the effects of interest, we computed the following contrasts on subject-level for the blocks containing food stimuli: (1) main effect of food type (palatable $>$ unpalatable and unpalatable $>$ palatable), (2) main effect of attentional focus (hedonic focus $>$ neutral focus and neutral focus $>$ hedonic focus), and (3) food type * attentional focus interaction. In previous fMRI studies on food reward (Martin et al., 2010; Rothemund et al., 2007; Stoeckel et al., 2008; Thomas et al., 2015), high caloric palatable food stimuli were often compared to neutral images in a so-called passive viewing paradigm. Therefore, we also computed contrasts to compare our palatable and unpalatable food conditions to neutral stimuli. Each food condition was compared to the neutral stimulus condition with a neutral focus. This resulted in four additional contrasts: (1) palatable food - neutral focus $>$ neutral 
stimuli - neutral focus, (2) palatable food - hedonic focus $>$ neutral stimuli - neutral focus, (3) unpalatable food - neutral focus $>$ neutral stimuli - neutral focus and (4) unpalatable food - hedonic focus $>$ neutral stimuli - neutral focus. To test whether an effect of attentional focus would also be apparent for non-food neutral stimuli, the esthetics focus was compared to the neutral focus for the non-food neutral stimuli: which resulted in two contrasts: (1) neutral stimuli - esthetics focus $>$ neutral stimuli neutral focus and (2) neutral stimuli - neutral focus $>$ neutral stimuli - esthetics focus.

On the second level of the analysis, whole brain group effects were tested using a random effects analysis. The parameter estimate contrast images specified on subject level were analyzed with a one-sample $t$-test against zero for the main effects and with an F-test for the group interaction effect. To correct for multiple comparisons, cluster extent family-wise error (FWE) correction was applied to control for false positives at an alpha of 0.05 for each statistical map. The spatial extent threshold was determined by computing 1,000 Monte Carlo simulations using AFNI's AlphaSim (Cox, 1996). Clusters sizes were determined based on the whole-brain anatomical mask, the uncorrected group t-maps and F-map with voxel threshold of $p$ $<0.001$ and with use of the estimated smoothness (FWHMx FWHMy FWHMz) values as was fed into the GLM. This approach is considered a valid stringent correction method (Eklund et al., 2016; Woo et al., 2014). In this way, the cluster extent threshold was computed and set for each group statistical map. Corrected significant clusters were defined as functional regions of interest (fROI) for further analysis. We extracted individual averaged beta values per fROI to compare the mean beta values per condition using the MarsBar toolbox (http://marsbar.sourceforge.net/) in SPM. XJview (http://www.alivelearn.net/xjview/) was used to visually inspect the results and to derive the significant cluster information. To analyze condition differences between beta weights, $t$-tests were performed in SPSS.

\section{Multivariate fMRI analysis}

Multi-voxel pattern analysis (MVPA) was conducted to test whether food palatability could be decoded from multi-voxel patterns of activity, and whether decoding performance was higher in the hedonic than in the neutral attentional focus. Classification analyses were first conducted in fROIs derived from the univariate main effect of attentional focus (hedonic focus > neutral focus collapsed over food types), and second with a whole-brain searchlight across attentional foci, for each of the attentional foci separately, and for the difference between these foci. 


\section{Classification trials}

We performed classification analysis using functions of the CoSMo Multivariate Pattern Analysis (MVPA) toolbox (Oosterhof et al., 2016) in Matlab. Instead of smoothed preprocessed images, the unsmoothed preprocessed functional images were included. To optimize the input for MVPA, the GLM design matrix was slightly adjusted for each participant. Each condition block was set as a predictor separately, which resulted in 12 predictors for each run. The predictors (of interest, motion and mean intensity) were set as described earlier for the univariate GLM model.

\section{Classification classes}

Data partitions consisted of classes of palatable and unpalatable food trials across and within an attentional focus (focus hedonic or focus neutral). Unequal trials between training and test partitions were balanced using CoSMo MVPA built-in functions (Cox \& Savoy, 2003).

\section{Classification algorithm}

For calculating classification accuracies, a linear support vector machine (ISVM) was used as classification algorithm. The classifier was trained on trial data of 3 runs and was tested on the trials of the remaining run, following a leave-one-run-out cross-validation procedure, resulting in 4 repetitions. We employed a permutation test on subject-level in which condition labels were permuted 1,000 times in the training phase in order to build a distribution of classification accuracies under the null-hypothesis. The true prediction accuracy was subsequently ranked against this distribution to obtain the p-value.

\section{fROI analysis}

First, to examine palatability accuracy predictions, mean accuracies were tested against permutation outcomes, per attentional focus and per fROI (derived from univariate main effect attentional focus) using non-parametric Wilcoxon signed-rank tests. To compare mean classification accuracies between the two attentional foci, group classification accuracies per fROI were also non-parametrically tested between focus hedonic and focus neutral. To correct for multiple comparisons of the clusters tested, FDR correction was applied on cluster-level p-values. Statistical analyses for MVPA were performed in Matlab and SPSS. 


\section{Whole brain searchlight analysis}

For each participant, a whole brain searchlight decoding analysis was conducted with a radius of 3 voxels (i.e. $6 \mathrm{~mm}$ ) within both attentional foci. The searchlight analysis also used ISVM as a classifier algorithm. After the searchlight analysis, subject-level data was spatially smoothed with $6 \mathrm{~mm}$ FWHM Gaussian kernel for the group analysis. For group analysis, only voxels that showed 80 percent overlap across participants were included for further analyses, to exclude voxels with poor group overlap (i.e. voxels at/outside the cortex border). As searchlight analyses entails that accuracy maps could have inflated clusters of informative voxels, voxel-wise FDR correction is applied as a more suitable approach to control for the rate of false positives. Mean accuracies across and within attentional foci were non-parametrically tested against chance level (0.5) and were FDR corrected on voxel-level. To test whether prediction accuracies differed between the attentional foci, the hedonic versus neutral focus accuracy differences were non-parametrically tested against 0 .

\section{Results}

\section{Behavioral assessments}

\section{Hunger}

Although hunger ratings ranged between 0 and 65, on average participants reported low hunger ( $M=26.8$ out of $100, S D=20.7)$, and only 5 of 23 participants reported a hunger level between 50 and 65 . Time passed since eating was as instructed $(M=$ 147.4 minutes, $S D=54.3$ ).

\section{Palatability ratings}

The individually tailored palatable stimuli scored very high on palatability ( $M=9.28$, $S D=0.5)$, whereas the unpalatable ones scored very low $(M=2.50, S D=1.42)$ on a 10-points scale, and they differed significantly ( $\left.t_{22}=20.70, p<0.001, d=4.317\right)$, showing that individual stimulus selection was successful (see also Supplementary Table 3.).

\section{Perceptual ratings}

Participants rated the similarity $(1=$ not similar at all to $5=$ very similar $)$ of shape and 
color of all the presented stimuli in an offline task. Within-category similarity (color: $M=1.91, S D=0.40$, shape: $M=1.81, S D=0.35$ ) was slightly higher than betweencategory similarity (color: $M=1.78, S D=0.41$, shape: $M=1.53, S D=0.35$ ), color: $t_{22}$ $=2.47, p=0.018, d=0.533$, shape: $t_{22}=5.94, p<0.001, d=1.235$. Overall, stimuli were rather dissimilar in terms of color and shape (i.e., all mean scores were $\leq 2.5$ ). For analyses of perceptual differences between the food and neutral categories, see supplementary Table 1. Importantly, all food stimuli used in the experiment were individualized (and therefore different across participants), which makes it unlikely that these slight visual perceptual differences between within-category and betweencategory influenced neural response to the different categories of stimuli.

\section{Task difficulty}

First, we examined the differences between conditions involved in our main fMRI analyses (food type and attentional focus). The food type (palatable vs unpalatable) * attentional focus (hedonic vs neutral) interaction was not significant in a 2-way ANOVA on response latencies $\left(F_{1,22}=0.138, p=0.713, \eta_{p}^{2}=0.006\right)$. Main effects of attentional focus $\left(F_{1,22}=1.450, p=0.241, \eta_{p}^{2}=0.062\right)$ or food type $\left(F_{1,22}=0.001, p=0.970, \eta_{p}^{2}\right.$ $<0.001)$ were not significant either. Next, we compared food-blocks to the neutral baseline condition (neutral stimuli - neutral focus). The 1-back task for the neutral baseline condition proved to be somewhat easier $(M=726.26 \mathrm{~ms}, S D=149.94)$ than the palatable food - neutral focus $\left(M=777.59 \mathrm{~ms}, S D=146.97 ; t_{22}=3.72, p=0.001\right.$, $d=0.776)$, palatable food - hedonic focus $\left(M=794.33 \mathrm{~ms}, S D=160.29 ; t_{22}=3.81, p\right.$ $=0.001, d=0.795)$, unpalatable food - neutral focus $\left(M=774.45 \mathrm{~ms}, S D=156.00 ; t_{22}\right.$ $=2.47, p=0.022, d=0.516)$ and unpalatable food - hedonic focus ( $M=796.46 \mathrm{~ms}$, $S D=167.41 ; t_{22}=4.19, p<0.001, d=0.874$ ) conditions. Furthermore, there were no significant differences in response latencies between the neutral baseline and the neutral stimuli with aesthetic focus conditions $\left(M=732.60 \mathrm{~ms}, S D=153.33 ; t_{22}=5.30\right.$, $p=0.601, d=0.111$ ).

\section{Whole-brain univariate analyses}

The main effect of food type (palatable versus unpalatable) was not significant after correction (cluster extent threshold set to 102). When examining the uncorrected t-contrasts maps ( $p=0.001$ cluster extent threshold: 10) of the main effect of food type, no robust clusters were found either. With this very lenient threshold (Eklund et al., 2016), the contrast palatable foods > unpalatable foods resulted in four 
relatively small clusters in the right cerebellum $\left(t_{22}=4.38\right.$, cluster size 11 ; peak MNI coordinates: $36,-62,-40)$, right inferior parietal lobe $\left(t_{22}=4.41\right.$, cluster size 29 ; peak MNI coordinates: $66,-34,24)$, left middle temporal gyrus: $t_{22}=4.33$, cluster size 13; peak MNI coordinates: $-52,-66,20$ ) and in the left mid occipital lobe: $t_{22}=4.10$, cluster size: 23; peak MNI coordinates: $-40,-70,34)$ and the contrast unpalatable foods $>$ palatable foods in two small clusters: in the right fusiform gyrus $\left(t_{22}=3,74\right.$, cluster size: 14 ; peak MNI coordinates: $38,-44,-24$ and $t_{22}=4.65$, cluster size: 17 ; peak MNI coordinates: 44, -66, -20). Note that clusters found with this lenient threshold can likely be for approximately $70 \%$ attributed to noise (Eklund et al., 2016).

Interestingly, neural activity was significantly higher for the hedonic focus than for the neutral focus when collapsing over food type (palatable vs unpalatable). For the hedonic > neutral focus contrast, five clusters were found (cluster extent threshold: 191 voxels). These clusters were found in the bilateral inferior parietal lobules, left superior frontal gyrus (dorsolateral prefrontal cortex (dIPFC)), bilateral precuneus/ posterior cingulate cortex and medial OFC. So, while the exact same visual food stimuli were presented, neural responding was stronger for a hedonic focus than for a neutral focus (see Table 2 and Figure $1 \mathrm{~b}$ ). The neutral $>$ hedonic focus contrast did not result in any significant clusters.

To test if the palatable - unpalatable contrast depended on attentional focus, we tested the food type * attentional focus interaction. A significant interaction effect in the left occipital lobe (cluster extent threshold: 131 voxels) was observed (see Table 1 and Figure 1a.).

Although the food type * attentional focus interaction was not statistically significant in any of our hypothesized brain areas, we tested the contrast palatable versus unpalatable foods (in both directions) in the hedonic focus and in the neutral focus condition separately whole-brain. These additional analyses were done to give the hypothesis of a palatability-dependent response (i.e., stronger response to palatable than to unpalatable foods) one more chance. Only the unpalatable $>$ palatable food contrast in the hedonic focus condition yielded a significant cluster (cluster extent threshold: 104 voxels), which was in the left occipital lobe (peak MNI coordinates: $-12,-90,-8)$ with a cluster size of 186 voxels $\left(t_{22}=4.84 p=0.005\right.$, data not shown in Figure). So, in this analysis, no evidence for a palatability-dependent neural response was found either. 
Because in previous studies neural responses to food cues were often compared to non-food items (Martin et al., 2010; Rothemund et al., 2007; Stoeckel et al., 2008), we compared the food conditions to a neutral baseline condition (neutral stimuli - neutral focus). The contrast palatable foods - neutral focus against neutral baseline (cluster size threshold: 110 voxels) showed activity in the left frontal mid OFC and the left inferior temporal gyrus. The contrast palatable foods - hedonic focus against neutral baseline (cluster size threshold: 174 voxels) showed an increased activity in the right inferior parietal lobule. The unpalatable foods - hedonic focus against neutral baseline (cluster size threshold: 188 voxels) showed an increased activity in the right posterior cingulate cortex, right inferior parietal lobule and right superior frontal gyrus (dIPFC) (See Table 1 and Figure 2). Contrasting unpalatable foods - neutral focus against baseline yielded no significant clusters. In sum, besides activity in the left lateral OFC in the palatable food - neutral focus condition, no response to the food versus neutral baseline conditions in reward-associated brain regions was found.

To test if there was a comparable effect of attentional focus for the neutral non-food stimuli as for the food stimuli, we examined the effect of attentional focus for the neutral stimuli (contrasts: esthetics focus $>$ neutral focus and neutral focus $>$ esthetics focus). No clusters reached significance after cluster threshold extent correction (cluster size threshold: 131).

To exclude the possibility that age, BMI and hunger levels affected neural responding in our study, we also entered these as covariates in our fMRI univariate group analyses. No significant contribution of any of these factors were found, and the originally observed effects remained. 
Table 1. Significant clusters from mass-univariate analyses including food type and attentional focus. Clusterletters corresponding to Figure 1. Initial $p<0.001$ maps were corrected with cluster extent threshold derived from Monte Carlo simulations.

\begin{tabular}{|c|c|c|c|c|c|}
\hline Cluster & $\begin{array}{l}\text { Anatomical } \\
\text { region }\end{array}$ & Hemisphere & $\begin{array}{l}\text { Clustersize } \\
\text { (num. } \\
\text { of } \\
\text { voxels) }\end{array}$ & $\begin{array}{c}\text { peak MNI } \\
\text { coordinates } \\
x(\mathrm{~mm}) y(\mathrm{~mm}) z(\mathrm{~mm})\end{array}$ & $\begin{array}{l}\text { peak cluster } \\
\text { F/t- P-value } \\
\text { value }\end{array}$ \\
\hline
\end{tabular}

Effects of food type and attentional focus

Interaction food * attentional focus
A Occipital Lobe
139
$\begin{array}{lll}-22 & -92 & -6\end{array}$
$28.53 \quad 0.012$

Main-effect attentional focus (hedonic focus > neutral focus)

\begin{tabular}{|c|c|c|c|c|c|c|c|c|}
\hline $\mathbf{E}$ & $\begin{array}{l}\text { Precuneus/ } \\
\text { Posterior } \\
\text { Cingulate Cortex }\end{array}$ & B & 2173 & -6 & -64 & 30 & 7.66 & $<0.001$ \\
\hline C & $\begin{array}{l}\text { Inferior Parietal } \\
\text { Lobule }\end{array}$ & $\mathrm{R}$ & 879 & 42 & -68 & 38 & 6.43 & $<0.001$ \\
\hline D & $\begin{array}{l}\text { Superior Frontal } \\
\text { Gyrus (dIPFC) }\end{array}$ & L & 559 & 24 & 36 & 54 & 5.20 & $<0.001$ \\
\hline B & $\begin{array}{l}\text { Inferior Parietal } \\
\text { Lobule }\end{array}$ & L & 442 & -44 & -70 & 30 & 4.88 & $<0.001$ \\
\hline $\mathbf{F}$ & $\begin{array}{l}\text { Medial } \\
\text { Orbitofrontal } \\
\text { Cortex }\end{array}$ & B & 265 & 8 & 56 & -6 & 5.57 & 0.001 \\
\hline
\end{tabular}

Food type versus neutral baseline

Palatable food - neutral focus > Neutral baseline

\begin{tabular}{|c|c|c|c|c|c|c|c|}
\hline $\begin{array}{l}\text { Cerebellum } \\
\text { Posterior Lobe }\end{array}$ & $\mathrm{R}$ & 444 & -2 & -76 & -18 & 5.39 & $<0.00$ \\
\hline $\begin{array}{l}\text { Cerebellum } \\
\text { Posterior Lobe }\end{array}$ & L & 244 & -22 & -70 & -26 & 5.73 & 0.003 \\
\hline $\begin{array}{l}\text { Lateral } \\
\text { Orbitofrontal } \\
\text { Cortex }\end{array}$ & L & 187 & -22 & 34 & -18 & 5.52 & $<0.00$ \\
\hline Inferior & L & 166 & -46 & -60 & -6 & 4.78 & 0.005 \\
\hline
\end{tabular}

$\begin{array}{lllllllll}\text { H } & \text { Inferior } & \text { L } & 166 & -46 & -60 & -6 & 4.78 & 0.005\end{array}$

Temporal Gyrus

Palatable food - hedonic focus > Neutral baseline

$\begin{array}{llllllll}\text { I Inferior Parietal } & R & 380 & 54 & -62 & 30 & 4.80 & <0.001\end{array}$

Lobule

Unpalatable food - hedonic focus $>$ Neutral baseline

\begin{tabular}{llllllll} 
Cerebellum & $\mathrm{L}$ & 797 & -28 & -70 & -20 & 6.20 & $<0.001$ \\
Posterior Lobe & & & & & & & \\
\hline
\end{tabular}


Table 1. (Continued)

\begin{tabular}{|c|c|c|c|c|c|c|c|c|}
\hline \multirow[t]{2}{*}{ Cluster } & \multirow[t]{2}{*}{$\begin{array}{l}\text { Anatomical } \\
\text { region }\end{array}$} & \multirow{2}{*}{ Hemisphere } & \multirow{2}{*}{$\begin{array}{l}\text { Clustersize } \\
\text { (num. } \\
\text { of } \\
\text { voxels) }\end{array}$} & \multicolumn{3}{|c|}{$\begin{array}{l}\text { peak MNI } \\
\text { coordinates }\end{array}$} & \multirow{2}{*}{$\begin{array}{c}\text { peak } \\
\text { F/t- } \\
\text { value }\end{array}$} & \multirow{2}{*}{$\begin{array}{l}\text { cluster } \\
\text { P-value }\end{array}$} \\
\hline & & & & $x(\mathrm{~mm})$ & $y(m m)$ & $\mathrm{z}(\mathrm{mm})$ & & \\
\hline J & $\begin{array}{l}\text { Inferior Parietal } \\
\text { Lobule }\end{array}$ & $\mathrm{R}$ & 568 & 48 & -62 & 38 & 6.06 & $<0.001$ \\
\hline $\mathbf{K}$ & $\begin{array}{l}\text { Superior Frontal } \\
\text { Gyrus (dIPFC) }\end{array}$ & $\mathrm{R}$ & 405 & 26 & 18 & 34 & 5.99 & $<0.001$ \\
\hline $\mathbf{L}$ & Cingulate Gyrus & $\mathrm{R}$ & 347 & 6 & -54 & 28 & 6.74 & $<0.001$ \\
\hline
\end{tabular}

Abbreviations: $\mathrm{L}=$ left, $\mathrm{R}=$ right, $\mathrm{B}=$ Bilateral, $\mathrm{MNI}=$ Montreal Neurological Institute, $\mathrm{dIPFC}=$ dorsolateral prefrontal cortex. 
a. Interaction effect: attentional focus $X$ food type

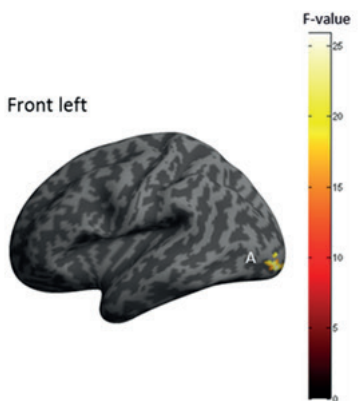

A. Left Occipital Lobe
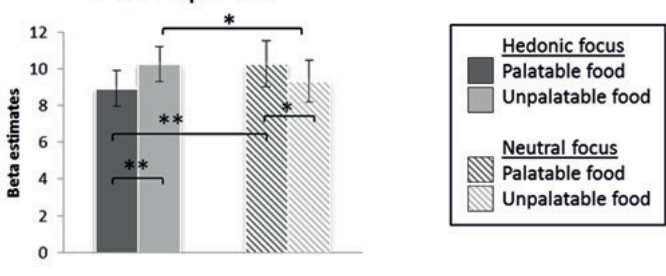

b. Main effect attentional focus: hedonic focus $>$ neutral focus
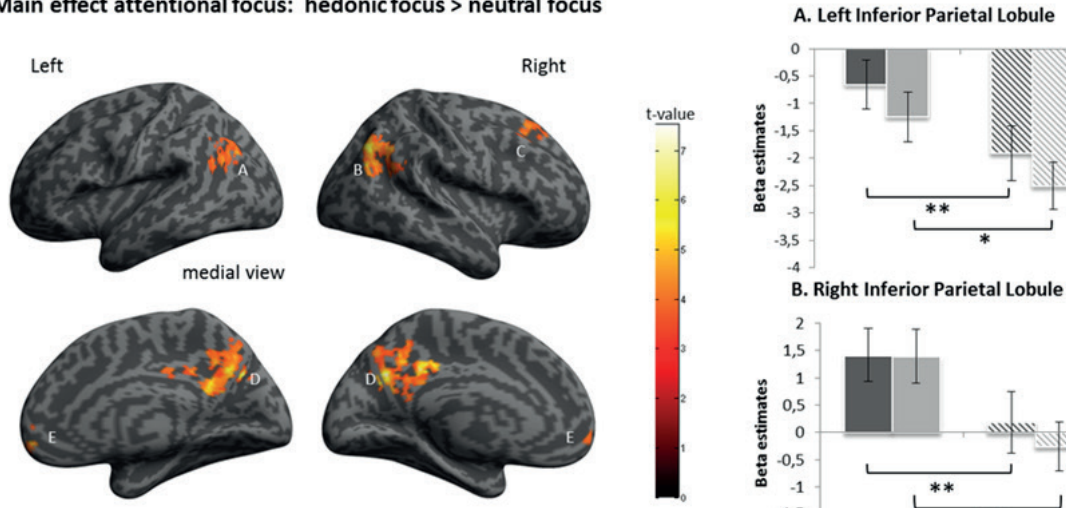

C. Right Superior Frontal Gyrus D. Precuneus / Cingulate Gyrus

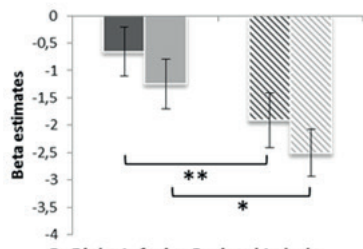

B. Right Inferior Parietal Lobule
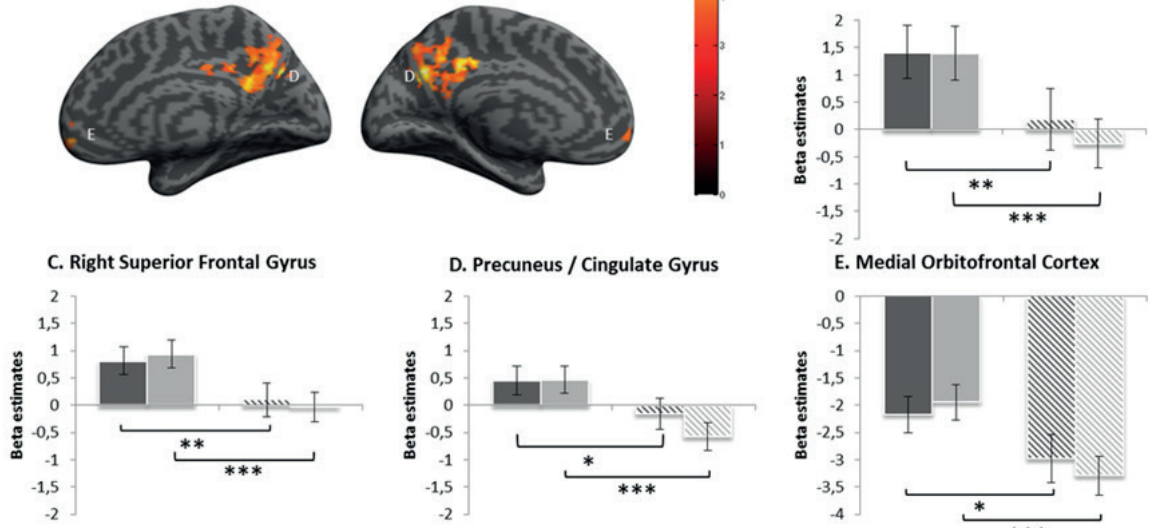

E. Medial Orbitofrontal Cortex

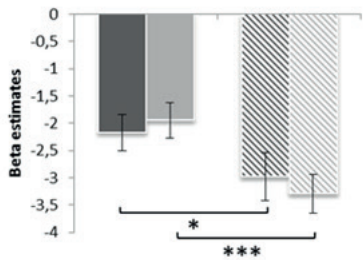

Figure 1. Results from mass-univariate analyses including food type and attentional focus. a. F-map of significant attentional focus * food type interaction. b. $t$-maps of significant main effect of attentional focus; were hedonic focus $>$ neutral focus collapsed over food type (palatable and unpalatable). a. and b., Bar plots represents mean extracted beta values with error bars (standard error of the mean) for each of the contribruting conditions per cluster. Statistical significance brackets indicate: $*=p<0.05 ; * \star=p<0.01$ and $* \star *=p<0.001$ as analysed with $t$-tests. 
a. Palatable food-neutral focus $>$ Neutral control- neutral focus
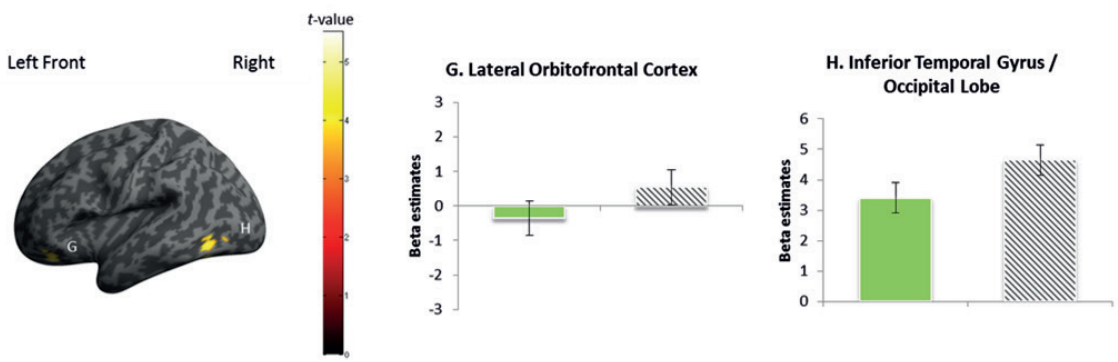

b. Palatable food-hedonic focus > Neutral control - neutral focus
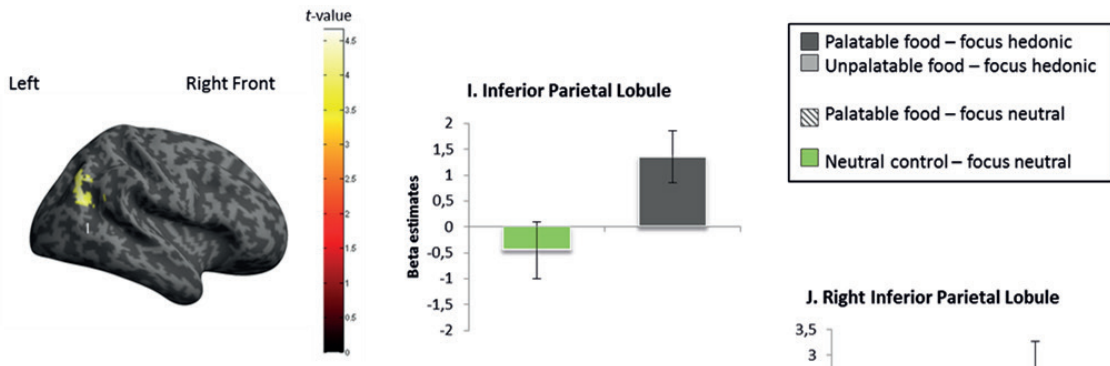

c. Unpalatable food-hedonic focus $>$ Neutral control- neutral focus

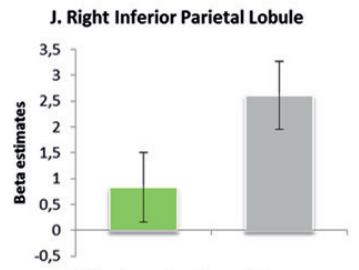

Left

Right

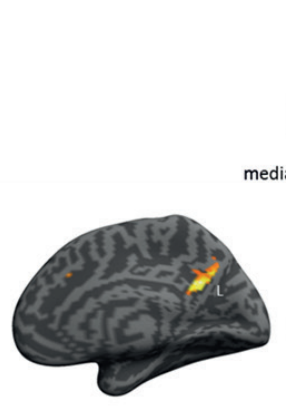

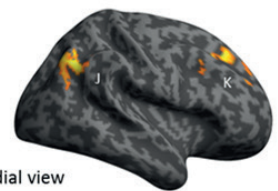

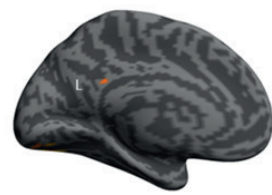

K. Right Superior Frontal Gyrus

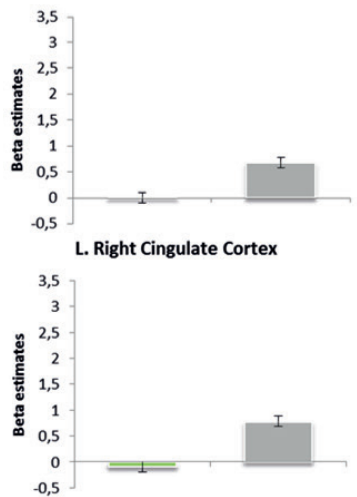

Figure 2. Results from mass-univariate analyses comparing food conditions to a neutral baseline condition a. $t$-map of significant clusters for palatable food - neutral focus. b. $t$-map of significant cluster for palatable food - hedonic focus, c. $t$-map of significant cluster for unpalatable food - hedonic focus $\mathbf{a}$, b. and $\mathbf{c}$., Bar plots represents mean extracted beta values with error bars (standard error of the mean) for each of the contribruting condition per cluster. 


\section{Classification of food palatability in functional regions of interest}

We first ran MVPA in the five functional regions of interest (fROI) derived from the univariate results of the main effect of attentional focus. Note here that the functional regions of interest were based on a different contrast (hedonic focus versus neutral focus) than the contrast relevant for our MVPA classification analysis (food type: palatable vs unpalatable), so avoiding the risk of double-dipping (Kriegeskorte et al., 2009). Food palatability could not be significantly decoded above chance in any of the fROIs (all comparisons $p>0.05$, FDR-corrected).

\section{Whole-brain classification of food palatability}

Using a whole-brain searchlight approach (Kriegeskorte et al., 2006), we tested whether food palatability could be decoded from patterns of neural activity and if this decoding accuracy was higher in the hedonic than in the neutral focus condition. Food palatability was significantly represented in multiple brain regions across attentional foci. Significant clusters (FDR corrected, with cluster size threshold: 5 for display purposes) included the right putamen, bilateral insula, caudate, right anterior cingulate and bilateral dorsolateral and medial prefrontal cortices. Also, in the hedonic focus condition, food palatability could be decoded in these areas. In contrast, in the neutral focus condition, food palatability could be decoded only in the dorsal bilateral prefrontal cortices and other non-reward related brain areas (see Figure 3 and Table 2 for all relevant cluster information). 


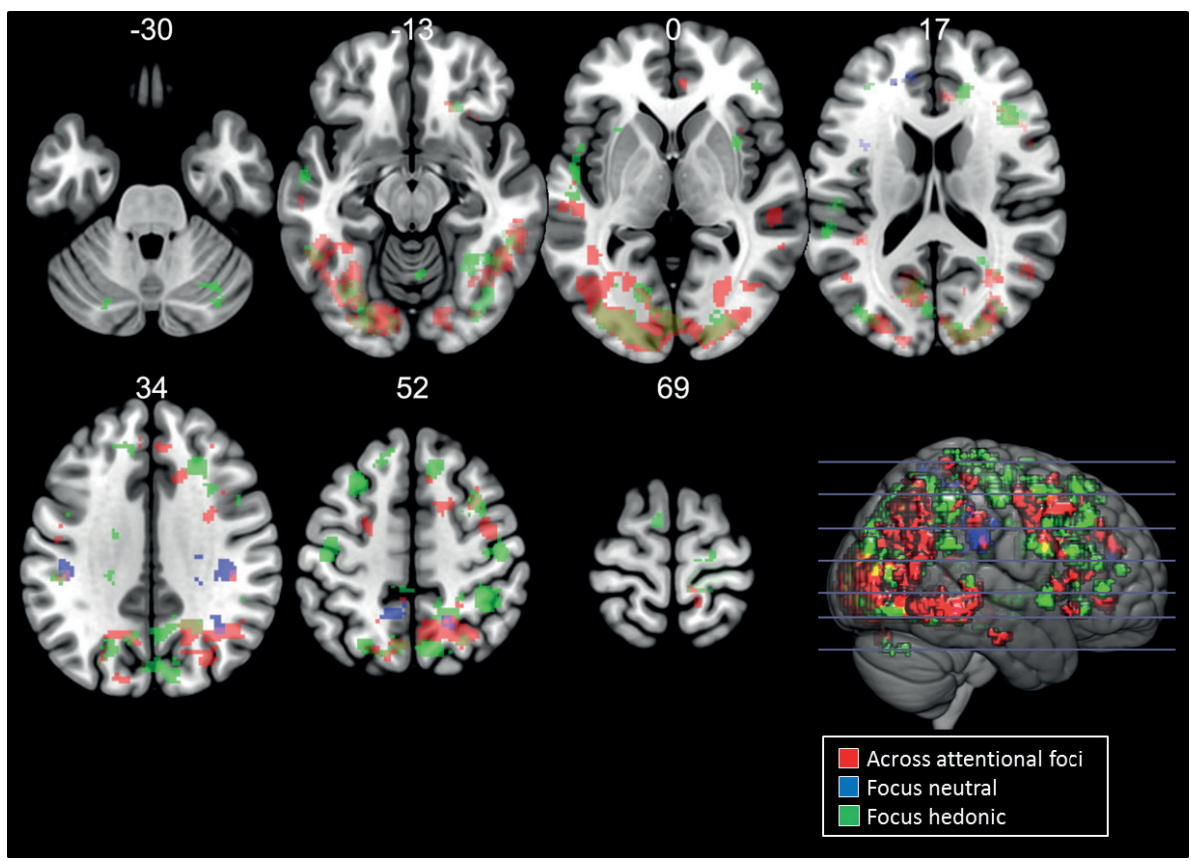

Figure 3. Neural representation of food palatablility. Clusters with significant classification accuracy of food palatability as derived from whole brain searchlight analysis across attentional foci and for the hedonic and neutral focus seperately. Clusters are presented on axial slices with the MNI Z-coordinates. These significant accuracy clusters were tested against $50 \%$ chance with Wilcoxon signed rank tests and FDR corrected $(p<0.05$, FDR corrected, clustersize threshold: 5 for display purposes). 
Table 2. Neural representation of food palatablility in reward-related areas. Clusters with significant classification accuracy of food palatability as derived from whole brain searchlight analysis in reward-related (mesocorticolimbic) brain areas across attentional foci and for the hedonic and neutral focus seperately. These significant accuracy clusters were tested against $50 \%$ chance with Wilcoxon signed rank tests and FDR corrected $(p<0.05$, FDR corrected, clustersize threshold: 5 for display purposes).

\begin{tabular}{|c|c|c|c|c|c|c|c|}
\hline Cluster & $\begin{array}{l}\text { Anatomical } \\
\text { region }\end{array}$ & Hemisphere & $\begin{array}{l}\text { Clustersize } \\
\text { (num. of } \\
\text { voxels) }\end{array}$ & $\mathrm{x}(\mathrm{mm}$ & $\begin{array}{l}\text { peak MN } \\
\text { oordinat }\end{array}$ & $\begin{array}{l}\text { VI } \\
\text { tes } \\
z(\mathrm{~mm})\end{array}$ & $\begin{array}{c}\text { Percentage } \\
\text { accuracy }\end{array}$ \\
\hline \multicolumn{8}{|c|}{ Across attentional foci (hedonic focus + neutral focus) } \\
\hline 1 & $\begin{array}{l}\text { Middle Frontal } \\
\text { Gyrus / Superior } \\
\text { Frontal Gyrus } \\
\text { (dIPFC) }\end{array}$ & $\mathrm{R}$ & 396 & 32 & 2 & 40 & 55.34 \\
\hline 2 & $\begin{array}{l}\text { Inferior Frontal } \\
\text { Gyrus incl. Insula } \\
\text { (43 vox.) }\end{array}$ & $\mathrm{R}$ & 378 & 50 & 8 & 26 & 56.17 \\
\hline 3 & $\begin{array}{l}\text { Middle Frontal } \\
\text { Gyrus (dIPFC) }\end{array}$ & L & 121 & -24 & -2 & 50 & 54.93 \\
\hline 4 & $\begin{array}{l}\text { Superior Frontal } \\
\text { Gyrus / Medial } \\
\text { Frontal Gyrus } \\
\text { (dIPFC) }\end{array}$ & $\mathrm{R}$ & 81 & 16 & 6 & 52 & 54.21 \\
\hline 5 & $\begin{array}{l}\text { Medial Frontal } \\
\text { Gyrus (dIPFC) }\end{array}$ & L & 74 & -12 & 46 & 24 & 54.91 \\
\hline 6 & Anterior Cingulate & $\mathrm{R}$ & 68 & 16 & 22 & 34 & 55.19 \\
\hline 7 & Anterior Cingulate & $\mathrm{R}$ & 61 & 8 & 44 & -2 & 54.23 \\
\hline 8 & $\begin{array}{l}\text { Medial Frontal } \\
\text { Gyrus (dIPFC) }\end{array}$ & $\mathrm{R}$ & 48 & 4 & 36 & 34 & 53.83 \\
\hline 9 & $\begin{array}{l}\text { Inferior Parietal } \\
\text { Lobule incl. Insula } \\
\text { (13 vox.) }\end{array}$ & L & 39 & -42 & -42 & 18 & 54.00 \\
\hline 10 & $\begin{array}{l}\text { Frontal Lobe / } \\
\text { OFC }\end{array}$ & $\mathrm{R}$ & 31 & 22 & 26 & -10 & 54.31 \\
\hline 11 & Anterior Cingulate & $\mathrm{R}$ & 30 & 6 & 34 & 16 & 53.94 \\
\hline 12 & $\begin{array}{l}\text { Inferior Frontal } \\
\text { Gyrus incl. Insula } \\
\text { (13 vox.) }\end{array}$ & $R$ & 28 & 30 & 22 & -10 & 53.65 \\
\hline 13 & Anterior Cingulate & L & 21 & -8 & 18 & 40 & 53.34 \\
\hline 14 & Anterior Cingulate & L & 7 & -10 & 30 & 30 & 53.19 \\
\hline 15 & $\begin{array}{l}\text { Superior Frontal } \\
\text { Gyrus (dIPFC) }\end{array}$ & $\mathrm{R}$ & 7 & 32 & 38 & 34 & 53.17 \\
\hline
\end{tabular}


Table 2. (Continued)

\begin{tabular}{|c|c|c|c|c|c|c|c|}
\hline Cluster & $\begin{array}{l}\text { Anatomical } \\
\text { region }\end{array}$ & Hemisphere & $\begin{array}{l}\text { Clustersize } \\
\text { (num. of } \\
\text { voxels) }\end{array}$ & $\begin{array}{r}p \\
\operatorname{coc} \\
x(\mathrm{~mm})\end{array}$ & $\begin{array}{l}\text { eak MN } \\
\text { ordinat }\end{array}$ & $\begin{array}{l}\text { VI } \\
\text { tes } \\
z(\mathrm{~mm})\end{array}$ & $\begin{array}{c}\text { Percentage } \\
\text { accuracy }\end{array}$ \\
\hline 16 & $\begin{array}{l}\text { Frontal Lobe } \\
\text { (dIPFC) }\end{array}$ & $L$ & 6 & -34 & 38 & 12 & 52.80 \\
\hline 17 & Insula & L & 5 & -44 & 10 & 4 & 52.91 \\
\hline \multicolumn{8}{|c|}{ Hedonic focus } \\
\hline 18 & $\begin{array}{l}\text { Middle/Inferior } \\
\text { Frontal Gyrus }\end{array}$ & $\mathrm{R}$ & 502 & 44 & 24 & 20 & 58.72 \\
\hline 19 & $\begin{array}{l}\text { Postcentral Gyrus } \\
\text { - incl. Insula ( } 27 \\
\text { vox.) }\end{array}$ & L & 370 & -50 & -20 & -48 & 55.61 \\
\hline 20 & $\begin{array}{l}\text { Middle Frontal } \\
\text { Gyrus (dIPFC) }\end{array}$ & L & 307 & -30 & 16 & 52 & 56.95 \\
\hline 21 & $\begin{array}{l}\text { Superior/Middle } \\
\text { Frontal Gyrus } \\
\text { (dIPFC) }\end{array}$ & $\mathrm{R}$ & 154 & 24 & 26 & 38 & 56.35 \\
\hline 22 & $\begin{array}{l}\text { Inferior Frontal } \\
\text { Gyrus }\end{array}$ & $\mathrm{R}$ & 78 & 54 & 10 & 20 & 55.53 \\
\hline 23 & $\begin{array}{l}\text { Inferior Frontal } \\
\text { Gyrus incl. } \\
\text { Caudate (9 vox.) }\end{array}$ & $\mathrm{R}$ & 73 & 22 & 28 & -6 & 57.47 \\
\hline 24 & Anterior Cingulate & $\mathrm{R}$ & 70 & 16 & 36 & 16 & 55.95 \\
\hline 25 & $\begin{array}{l}\text { Superior Frontal } \\
\text { Gyrus (dIPFC) }\end{array}$ & L & 53 & -16 & 28 & 54 & 56.08 \\
\hline 26 & $\begin{array}{l}\text { Insula (33 vox.) - } \\
\text { Putamen (20 vox.) }\end{array}$ & $\mathrm{R}$ & 52 & 32 & 10 & -2 & 56.33 \\
\hline 27 & $\begin{array}{l}\text { Middle/Inferior } \\
\text { frontal Gyrus }\end{array}$ & $\mathrm{R}$ & 44 & 46 & 10 & 36 & 55.05 \\
\hline 28 & $\begin{array}{l}\text { Inferior frontal } \\
\text { Gyrus }\end{array}$ & $\mathrm{R}$ & 32 & 44 & 38 & 0 & 55.60 \\
\hline 29 & $\begin{array}{l}\text { Superior Frontal } \\
\text { Gyrus (dIPFC) }\end{array}$ & L & 30 & -20 & 36 & 36 & 54.97 \\
\hline 30 & $\begin{array}{l}\text { Superior Frontal } \\
\text { Gyrus (dIPFC) }\end{array}$ & $\mathrm{R}$ & 17 & 24 & 48 & 12 & 56.17 \\
\hline 31 & $\begin{array}{l}\text { Inferior Frontal } \\
\text { Gyrus }\end{array}$ & L & 15 & -46 & 28 & 6 & 54.38 \\
\hline 32 & $\begin{array}{l}\text { Middle Frontal } \\
\text { Gyrus (dIPFC) }\end{array}$ & $\mathrm{R}$ & 15 & 34 & 24 & 48 & 54.95 \\
\hline 33 & $\begin{array}{l}\text { Middle Frontal } \\
\text { Gyrus (v/dIPFC) }\end{array}$ & L & 11 & -34 & 40 & 14 & 55.33 \\
\hline
\end{tabular}


Table 2. (Continued)

\begin{tabular}{|c|c|c|c|c|c|c|c|}
\hline Cluster & $\begin{array}{l}\text { Anatomical } \\
\text { region }\end{array}$ & Hemisphere & $\begin{array}{l}\text { Clustersize } \\
\text { (num. of } \\
\text { voxels) }\end{array}$ & $\begin{array}{r}\text { pe } \\
\text { coo } \\
x(\mathrm{~mm})\end{array}$ & $\begin{array}{l}\text { eak MN } \\
\text { ordinate } \\
y(\mathrm{~mm})\end{array}$ & $\begin{array}{l}\text { VI } \\
\text { tes } \\
z(\mathrm{~mm})\end{array}$ & $\begin{array}{c}\text { Percentage } \\
\text { accuracy }\end{array}$ \\
\hline 34 & Insula & $\mathrm{R}$ & 7 & 32 & 26 & 6 & 55.61 \\
\hline 35 & $\begin{array}{l}\text { Medial Frontal } \\
\text { Gyrus (dIPFC) }\end{array}$ & L & 6 & -12 & 26 & 40 & 53.70 \\
\hline 36 & Fusiform Gyrus & L & 5 & -38 & -42 & -18 & 53.81 \\
\hline 37 & Insula & L & 5 & -34 & 12 & 6 & 53.10 \\
\hline \multicolumn{8}{|c|}{ Neutral focus } \\
\hline 38 & $\begin{array}{l}\text { Medial Frontal } \\
\text { Gyrus (dIPFC) }\end{array}$ & L & 44 & -12 & 44 & 24 & 56.88 \\
\hline 39 & Frontal Lobe & $\mathrm{R}$ & 10 & 28 & -16 & 36 & 54.79 \\
\hline 40 & $\begin{array}{l}\text { Medial Frontal } \\
\text { Gyrus (v/dlPFC) }\end{array}$ & L & 5 & -22 & 40 & 16 & 54.02 \\
\hline
\end{tabular}

Abbreviations: $\mathrm{L}=$ left, $\mathrm{R}=$ right, $\mathrm{B}=$ Bilateral, $\mathrm{MNI}=$ Montreal Neurological Institute, $\mathrm{VLPFC}=$ ventrolateral prefrontal cortex, dIPFC = dorsolateral prefrontal cortex.

We then tested whether the decoding accuracies differed significantly between the two attentional foci. After voxel-wise FDR correction, no clusters remained significant. To exploratory inspect the differences between attentional foci, the mean accuracy difference map threshold was set at $p<0.001$. Subsequently, mean cluster accuracies of clusters with a significant difference between the attentional foci (hedonic vs neutral) were derived and non-parametrically tested against chance (0.5). To truthfully interpret this comparison map, only clusters where the mean accuracy of hedonic focus was significantly higher than 0.5 were reported as results. In seven clusters decoding accuracy was higher in the hedonic than in the neutral focus condition, whereas no clusters produced a higher decoding accuracy in the neutral than in the hedonic focus condition. So, food palatability could be decoded above chance, and this effect was mostly limited to the hedonic focus condition. Please see Figure 4. and Table 3. for these significant mean accuracies (plotted as percentages). 

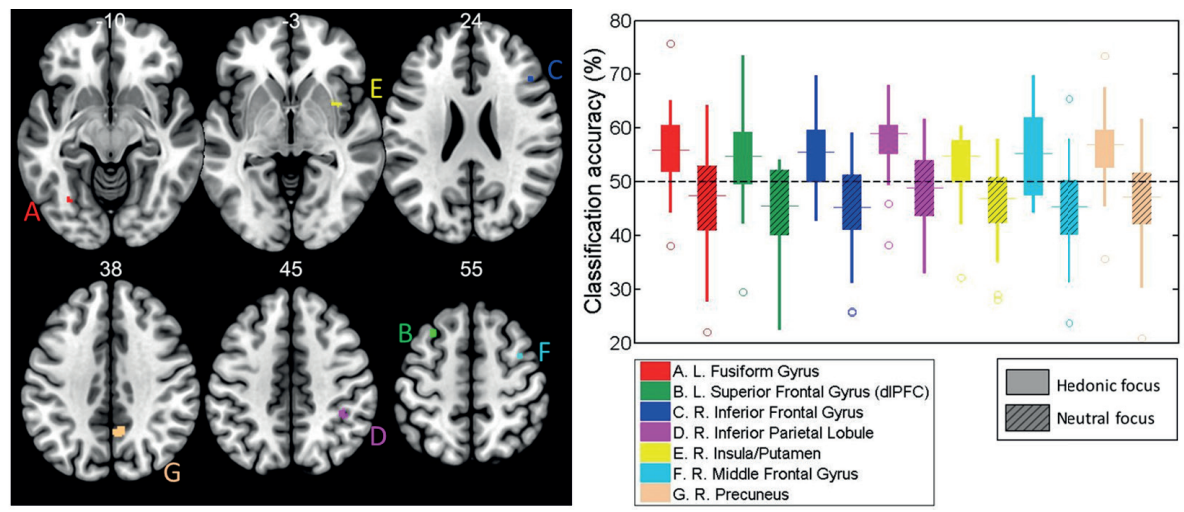

Figure 4. Differences in neural representation of food palatability between attentional foci. Clusters where classification accuracy decoding was significantly higher for the hedonic focus than for the neutral focus $(p<0.001)$. Clusters are presented on axial slices with the MNI Z-coordinates. Mean decoding accuracies for hedonic and neutral focus plotted as box and whisker plots for each cluster. The central line in plot represents the median, the bottom and top edges of the box indicate the $25^{\text {th }}$ and $75^{\text {th }}$ percentiles $\left(q_{25}\right.$ and $\left.q_{75}\right)$. The ends of the whiskers indicate the maximum and minimum accuracy values that were not considered to be outliers. Outliers (datapoints $<q_{25}-1.5 \times\left(q_{75}-q_{25}\right)$ or $>q_{75}+1.5 \times\left(q_{75}-q_{25}\right)$ ) are plotted as open circles. Abbreviations represents: $\mathrm{L}=$ left, $\mathrm{R}=$ right, $\mathrm{dIPFC}=$ dorsolateral prefrontal cortex

Table 3. Attentional focus difference in neural representation of food palatablility. Clusters with significant decoding accuracy differences (percentages) between hedonic focus and neutral focus as derived from whole brain searchlight analysis. These significant accuracy difference clusters were tested against zero with a Wilcoxon signed rank tests $(p<0.001)$. Clusterletters corresponding to Figure 2.

\begin{tabular}{llllllll}
\hline Cluster & \multicolumn{1}{c}{$\begin{array}{c}\text { Anatomical } \\
\text { region }\end{array}$} & $\begin{array}{c}\text { Hemisphere } \\
\text { Clustersize } \\
\text { (num. of } \\
\text { voxels) }\end{array}$ & $\begin{array}{c}\text { peak MNI } \\
\text { coordinates } \\
\text { MNI(x) }\end{array}$ & $\begin{array}{c}\text { MNI(y) } \\
\text { PNI(z) }\end{array}$ & $\begin{array}{c}\text { Percentage } \\
\text { accuracy }\end{array}$ \\
\hline difference
\end{tabular}

Abbreviations: $\mathrm{L}=$ left. $\mathrm{R}=$ right. $\mathrm{B}=$ Bilateral. $\mathrm{MNI}=$ Montreal Neurological Institute. $\mathrm{dIPFC}=$ dorsolateral prefrontal cortex. 


\section{Discussion}

The current study assessed the level and multi-voxel pattern of neural activity in response to high caloric visual food stimuli, and the role of attentional focus. The main conclusions from the univariate analyses include: (1) the level of neural activity was not significantly different between high-caloric palatable and high-caloric unpalatable food stimuli, (2) independent of food palatability, several relevant brain regions (e.g., medial OFC) responded more strongly with a hedonic focus than with a neutral focus, while - crucially - exactly the same visual food stimuli were presented. The main conclusions from the multivariate analyses include: (1) within fROls derived from the univariate main effect of attentional focus, food palatability could not be decoded above chance from neural patterns; (2) food palatability could be decoded above chance in several relevant brain regions (e.g., insula/putamen) resulting from a wholebrain searchlight, mostly in the hedonic focus condition.

Interestingly, hardly any significant differences were found between the level of neural activity elicited by palatable versus unpalatable high caloric foods (univariate analyses). In interaction with attentional focus, food palatability only led to differential activity in one cluster in the left occipital lobe. Here, neural activity was higher for palatable than unpalatable food stimuli in the neutral focus condition, while the reverse was observed in the hedonic focus condition. Because the occipital lobe is involved in processing of visual food cues (van der Laan et al., 2011), these results suggest that the visual saliency of the presented food stimuli may have depended on the attentional focus.

Importantly, the virtual lack of differences in the level of neural activity elicited by palatable versus unpalatable food stimuli cannot be attributed to limited differences in food palatability, as our food stimuli were individually tailored and the palatability ratings for palatable vs unpalatable foods were highly distinct. Note that many previous studies did not individually tailor food stimuli, and did not include unpalatable food stimuli (Pursey et al., 2014; Rothemund et al., 2007; Stoeckel et al., 2008; Yokum et al., 2011). We also compared the four food-conditions separately to a neutral non-food baseline condition to be in line with prior literature that often used this type of baseline (Martin et al., 2010; Rothemund et al., 2007; Stoeckel et al., 2008). Again, no clear evidence for reward-related brain activity specifically for palatable food was found. These findings cast doubt on the general and popular view that the level 
of neural activity elicited by high caloric food stimuli reflects the reward-value of these food stimuli, as in the current study the level of neural activity was not proportionate to the palatability of the presented foods. As most previous studies did not include unpalatable food stimuli, it was not possible in those studies to distinguish between saliency and valence. It might be the case that the level of activity in brain regions that have been associated with processing food-reward value (see for a review Volkow, Wang, \& Baler, 2011) reflects motivational saliency instead of motivational value (i.e. reward value), and highly palatable and highly unpalatable stimuli might be equally salient.

Attentional focus (hedonic > neutral) was strongly associated with a differential neural response to high-caloric visual food stimuli, independent of food palatability, in bilateral inferior parietal lobules, left superior frontal gyrus, bilateral precuneus/ posterior cingulate cortex, and medial OFC. Most of these brain areas have been associated with visual food processing in previous (review) studies (Giuliani et al., 2018; Pohl et al., 2017; Suzuki et al., 2017; van der Laan et al., 2011). The precuneus and the posterior cingulate cortex were previously associated with an increased level of attention in visual palatable food processing in a cognitive modulation task (Yokum \& Stice, 2013). Furthermore, other studies have shown that activity in the medial OFC was associated with food reward as well as saliency (Rothkirch et al., 2012; Suzuki et al., 2017). These differences in neural responding cannot likely be attributed to betweencondition differences in task difficulty, because conditions did not differ significantly in response latency, which is an indication of perceptual task difficulty (Schneider et al., 2011). These findings illustrate that a hedonic versus a neutral attentional focus elicits distinct neural responses, while participants are processing exactly the same visual food stimuli, and independent of food palatability. Taken together, the level of activity in these brain regions primarily reflects the motivational saliency of highcaloric foods, which is stronger with a hedonic focus, and the level of neural activity is not proportionate to the palatability of these foods.

As univariate analyses are only informative regarding involvement of brain areas, but not regarding representational content (Norman et al., 2006), we also conducted multivariate analyses. Results from these analyses showed that food palatability could not be decoded from multi-voxel patterns within fROIs derived from the univariate main effect of attentional focus. Interestingly, food palatability could be decoded from several reward-related areas using a whole-brain searchlight approach, specifically 
with a hedonic focus. This shows that when examining neural patterns, palatable and unpalatable foods can be distinguished above chance, and also points to the importance of taking attentional focus into account. These results largely fit with prior research, which showed that subjective value could be decoded from multi-voxel patterns of neural activity, whereas the level of neural activity was not dependent on food palatability (Chikazoe et al., 2014; Suzuki et al., 2017). But unlike these studies, we could not decode food palatability in the lateral or medial OFC. The observed average decoding accuracy percentages correspond to those reported in another recent food valuation study using multivariate decoding (Suzuki et al., 2017). Taken together, our findings illustrate the importance of considering attentional focus, because the representational content mostly reflected palatability information when participants applied a hedonic focus during task performance. Note that significant decoding performance does not ultimately prove that a brain region is necessary for the mental process being decoded, as neuroimaging data is inherently correlational (Poldrack, 2011).

Strengths of the current study include (1) that we had tight experimental control over the mental process participants were engaged in while they were being scanned (Poldrack, 2011), (2) that we included individually tailored highly palatable and highly unpalatable food items, and (3) that we implemented both massunivariate as well as state-of-the-art multivariate analyses of fMRI data. A limitation is that our study did not include a healthy weight control group, as we were primarily interested in the effect of an applied attentional focus in this more sensitive group of people with overweight. Future research could address whether our findings generalize to men with overweight and to healthy-weight men and women, as well as include an attentional focus on health or caloric density. To optimize the multivariate classification analyses, more blocks of each type could be acquired in the future to be able to train the classifier better (Haynes, 2015).

\section{Conclusions}

In this study, we observed a significant effect of attentional focus on the level of neural activity elicited by high-caloric visual food stimuli in females with overweight. Mass-univariate analyses could hardly distinguish between palatable and unpalatable food stimuli, whereas a remarkable difference was found between a hedonic focus versus a neutral focus when collapsing over food palatability: several brain regions 
responded more strongly in a hedonic compared to neutral focus when processing exactly the same visual stimuli. As highly palatable and highly unpalatable food stimuli are similarly salient, but differ in reward value, and because a hedonic focus may highlight that saliency, this suggests that neural responses to visual food stimuli may reflect saliency instead of reward value. Interestingly, multivariate analyses showed that neural patterns could distinguish between palatable and unpalatable food stimuli and specifically in a hedonic attentional focus.

Previous studies on the neural correlates of the reward value of food are highly inconsistent (Ziauddeen et al., 2012), and the current study suggests that the lack of a well-controlled fMRI paradigm may have contributed to this inconsistency. Although more research is needed to confirm these findings, the hypothesis that the level of neural activity in response to high-caloric food stimuli is proportionate to the hedonic value of presented food stimuli in people with overweight should be reconsidered.

\section{Data and code availability}

The unthresholded statistical maps of the univariate analysis and searchlight classification accuracy maps are made available on Neurovault. The raw data and code that support the findings of this study are available from the corresponding author upon reasonable request.

\section{Acknowledgements}

This study was partly financed by the Netherlands Organization for Scientific Research (NWO), vici-grant (453.10.006) awarded to Anita Jansen, NWO vidi-grant (452.16.007) awarded to Anne Roefs and the Maastricht University Interfaculty Program 'Eatwell' awarded to Anita Jansen.

\section{Author Contributions}

S.F., Anne R. and A.J. designed the study. S.F. collected the data. S.F. and J.H analyzed the data. The scanning protocol was set up by Alard R. S.F. and Anne R. wrote the manuscript, A.J., Alard R., and J.H. gave feedback on the manuscripts, and all authors approved the final version. 


\section{Conflicts of interests}

The authors declare no conflicts of interest.

\section{Supplementary information}

Supplementary Figure 1. Example of 2 pictures (A and B) of 5 high caloric food stimuli. Pictures representing; 1: chocolate cookies, 2: pizza, 3: chocolate, 4: blue cheese and 5: bacon.

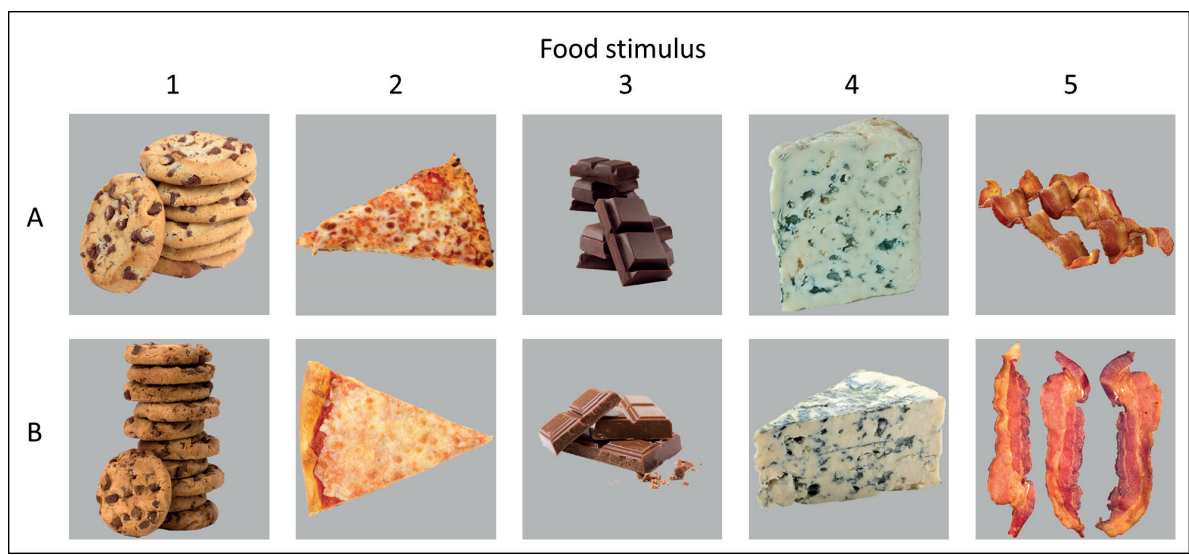

Supplementary Table 1. Within and between category visual similarities. Mean betweenand within-category similarities rating scores for all presented stimuli on color and shape, from 1 (not similar at all) to 5 (highly similar). Between categories were palatable food - unpalatable food stimuli, palatable food - neutral stimuli and unpalatable food - neutral stimuli and within categories were palatable food -palatable food stimuli, unpalatable food - unpalatable stimuli and neutral - neutral stimuli.

\begin{tabular}{|c|c|c|c|}
\hline \multicolumn{2}{|c|}{ Between-category similarity } & \multicolumn{2}{|c|}{ Within-category similarity } \\
\hline Color & mean score (SD) & Color & mean score (SD) \\
\hline $\begin{array}{l}\text { Palatable versus } \\
\text { unpalatable }\end{array}$ & $2.14(0.71)$ & $\begin{array}{l}\text { Palatable versus } \\
\text { palatable }\end{array}$ & $2.54(0.66)$ \\
\hline Palatable versus neutral & $1.67(0.30)$ & $\begin{array}{l}\text { Unpalatable versus } \\
\text { unpalatable }\end{array}$ & $1.99(0.66)$ \\
\hline $\begin{array}{l}\text { Unpalatable versus } \\
\text { neutral }\end{array}$ & $1.53(0.34)$ & Neutral versus neutral & $1.18(0.20)$ \\
\hline $\begin{array}{l}\text { Average between } \\
\text { category similarity }\end{array}$ & $1.78(0.42)$ & $\begin{array}{l}\text { Average within-category } \\
\text { similarity }\end{array}$ & $1.91(0.40)$ \\
\hline
\end{tabular}


Supplementary Table 1. (Continued)

\begin{tabular}{|c|c|c|c|}
\hline \multicolumn{2}{|c|}{ Between-category similarity } & \multicolumn{2}{|c|}{ Within-category similarity } \\
\hline Shape & & Shape & \\
\hline $\begin{array}{l}\text { Palatable versus } \\
\text { unpalatable }\end{array}$ & $1.77(0.44)$ & $\begin{array}{l}\text { Palatable versus } \\
\text { palatable }\end{array}$ & $1.69(0.42)$ \\
\hline Palatable versus neutral & $1.40(0.36)$ & $\begin{array}{l}\text { Unpalatable versus } \\
\text { unpalatable }\end{array}$ & $1.70(0.47)$ \\
\hline $\begin{array}{l}\text { Unpalatable versus } \\
\text { neutral }\end{array}$ & $1.42(0.40)$ & Neutral versus neutral & $2.05(0.59)$ \\
\hline $\begin{array}{l}\text { Average between } \\
\text { category similarity }\end{array}$ & $1.53(0.35)$ & $\begin{array}{l}\text { Average within-category } \\
\text { similarity }\end{array}$ & $1.81(0.35)$ \\
\hline
\end{tabular}

Abbreviation: $\mathrm{SD}$ = standard deviation

Supplementary Table 2. Neural representation of food palatablility in non-reward areas. Clusters with significant decoding accuracy of food palatability as derived from whole brain searchlight analysis not in reward-related (mesocorticolimbic) brain areas across attentional foci and for the hedonic and neutral focus seperately. These significant accuracy clusters were tested against $50 \%$ chance with Wilcoxon signed rank tests and FDR corrected ( $p$ $<0,05$, FDR corrected, clustersize threshold: 5 for display purposes).

\begin{tabular}{|c|c|c|c|c|c|c|c|}
\hline \multirow[t]{2}{*}{ Cluster } & \multirow[t]{2}{*}{$\begin{array}{l}\text { Anatomical } \\
\text { region }\end{array}$} & \multirow[t]{2}{*}{ Hemisphere } & \multirow{2}{*}{$\begin{array}{l}\text { Clustersize } \\
\text { (num. of } \\
\text { voxels) }\end{array}$} & \multicolumn{3}{|c|}{$\begin{array}{l}\text { peak MNI } \\
\text { coordinates }\end{array}$} & \multirow{2}{*}{$\begin{array}{l}\text { Percentage } \\
\text { Accuracy }\end{array}$} \\
\hline & & & & $x(\mathrm{~mm}$ & $y(\mathrm{~mm})$ & $\mathrm{z}(\mathrm{mm})$ & \\
\hline \multicolumn{8}{|c|}{$\begin{array}{l}\text { Across attentional foci } \\
\text { (hedonic focus + neutral focus) }\end{array}$} \\
\hline 1 & Occipital Lobe & $L$ & 9356 & -10 & -86 & -8 & 59.24 \\
\hline 2 & $\begin{array}{l}\text { Posterior } \\
\text { Cingulate / } \\
\text { Precuneus }\end{array}$ & L & 178 & -6 & -42 & 48 & 55.79 \\
\hline 3 & $\begin{array}{l}\text { Superior } \\
\text { Temporal Gyrus }\end{array}$ & L & 116 & -60 & -24 & -4 & 55.06 \\
\hline 4 & $\begin{array}{l}\text { Parietal Lobe incl. } \\
\text { Precuneus } \\
\text { (47 vox.) }\end{array}$ & L & 76 & -18 & -52 & 56 & 53.92 \\
\hline 5 & Postcentral Gyrus & $\mathrm{R}$ & 73 & 44 & -28 & 40 & 54.26 \\
\hline 6 & $\begin{array}{l}\text { Cerebellum } \\
\text { Posterior Lobe }\end{array}$ & L & 40 & -12 & -78 & -22 & 55.51 \\
\hline 7 & $\begin{array}{l}\text { Precentral Gyrus / } \\
\text { Supp. Motor Area }\end{array}$ & L & 38 & -48 & -6 & 38 & 53.76 \\
\hline 8 & Postcentral Gyrus & $\mathrm{R}$ & 38 & 34 & -34 & 60 & 53.52 \\
\hline 9 & $\begin{array}{l}\text { Inferior Temporal } \\
\text { Gyrus }\end{array}$ & $\mathrm{R}$ & 35 & 54 & -18 & -22 & 54.47 \\
\hline
\end{tabular}


Supplementary Table 2. (Continued)

\begin{tabular}{|c|c|c|c|c|c|c|c|}
\hline \multirow[t]{2}{*}{ Cluster } & \multirow[t]{2}{*}{$\begin{array}{l}\text { Anatomical } \\
\text { region }\end{array}$} & \multirow[t]{2}{*}{ Hemisphere } & \multirow{2}{*}{$\begin{array}{l}\text { Clustersize } \\
\text { (num. of } \\
\text { voxels) }\end{array}$} & \multicolumn{3}{|c|}{$\begin{array}{c}\text { peak MNI } \\
\text { coordinates }\end{array}$} & \multirow{2}{*}{$\begin{array}{c}\text { Percentage } \\
\text { Accuracy }\end{array}$} \\
\hline & & & & $x(\mathrm{~mm})$ & $y(\mathrm{~mm})$ & $\mathrm{z}(\mathrm{mm})$ & \\
\hline 10 & Postcentral Gyrus & $R$ & 32 & 10 & -38 & 70 & 53.52 \\
\hline 11 & Postcentral Gyrus & L & 27 & -44 & -30 & 34 & 53.58 \\
\hline 12 & Angular Gyrus & L & 25 & -48 & -60 & 28 & 53.48 \\
\hline 13 & $\begin{array}{l}\text { Supplemental } \\
\text { Motor Area }\end{array}$ & $\mathrm{R}$ & 24 & 12 & 22 & 46 & 53.60 \\
\hline 14 & $\begin{array}{l}\text { Superior } \\
\text { Temporal Gyrus }\end{array}$ & L & 23 & -54 & -44 & 12 & 54.36 \\
\hline 15 & $\begin{array}{l}\text { Supplemental } \\
\text { Motor Area }\end{array}$ & $\mathrm{R}$ & 17 & 10 & 16 & 46 & 53.31 \\
\hline 16 & Frontal Lobe & L & 13 & -22 & -24 & 38 & 53.43 \\
\hline 17 & $\begin{array}{l}\text { Posterior } \\
\text { Cingulate }\end{array}$ & $\mathrm{R}$ & 11 & 16 & -22 & 38 & 53.80 \\
\hline 18 & $\begin{array}{l}\text { Inferior Parietal } \\
\text { Lobule }\end{array}$ & $\mathrm{R}$ & 9 & 50 & -36 & 48 & 53.23 \\
\hline 19 & Parietal Lobe & $\mathrm{R}$ & 8 & 26 & -48 & 54 & 53.20 \\
\hline 20 & Precentral Gyrus & $\mathrm{R}$ & 7 & 40 & -18 & 54 & 52.68 \\
\hline 21 & Frontal Lobe & $\mathrm{L}$ & 5 & -14 & -16 & 48 & 53.18 \\
\hline
\end{tabular}

Hedonic focus

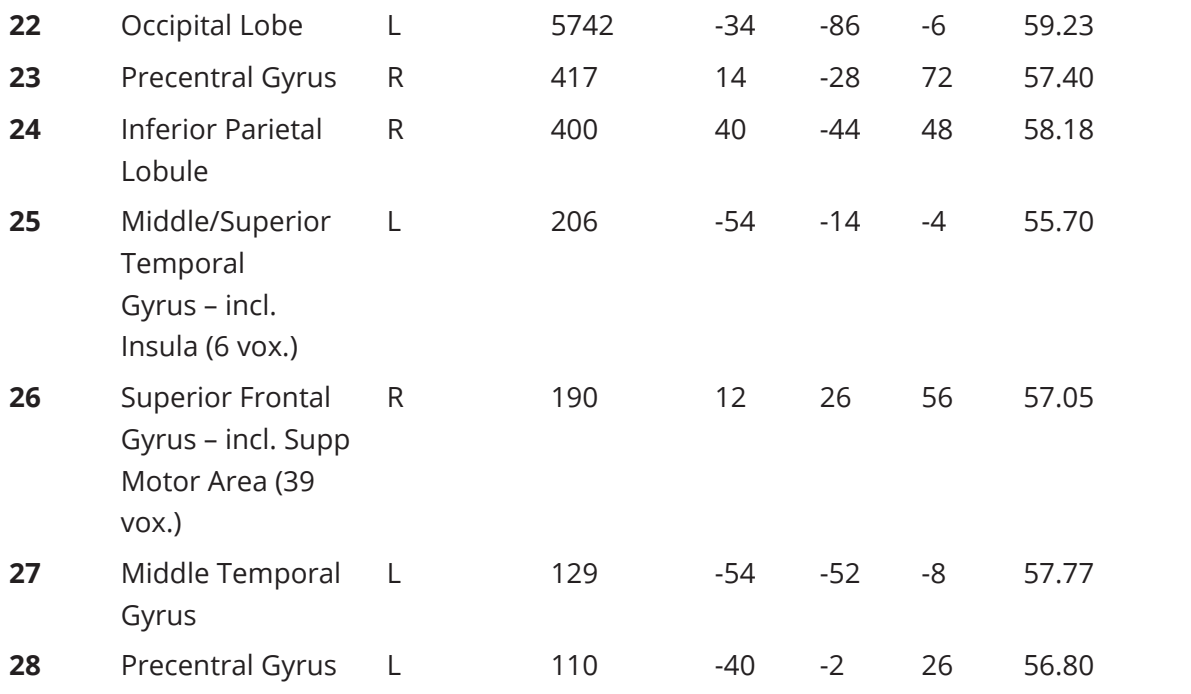


Supplementary Table 2. (Continued)

\begin{tabular}{|c|c|c|c|c|c|c|c|}
\hline \multirow[t]{2}{*}{ Cluster } & \multirow[t]{2}{*}{$\begin{array}{l}\text { Anatomical } \\
\text { region }\end{array}$} & \multirow[t]{2}{*}{ Hemisphere } & \multirow{2}{*}{$\begin{array}{l}\text { Clustersize } \\
\text { (num. of } \\
\text { voxels) }\end{array}$} & \multicolumn{3}{|c|}{$\begin{array}{c}\text { peak MNI } \\
\text { coordinates }\end{array}$} & \multirow{2}{*}{$\begin{array}{l}\text { Percentage } \\
\text { Accuracy }\end{array}$} \\
\hline & & & & $x(\mathrm{~mm})$ & $y(m m)$ & $\mathrm{z}(\mathrm{mm})$ & \\
\hline 29 & $\begin{array}{l}\text { Inferior Parietal } \\
\text { Lobule }\end{array}$ & $R$ & 109 & 52 & -44 & 26 & 57.08 \\
\hline 30 & Frontal Gyrus & L & 58 & -26 & -32 & 42 & 54.75 \\
\hline 31 & $\begin{array}{l}\text { Medial Frontal } \\
\text { Gyrus - Supp. } \\
\text { Motor Area }\end{array}$ & $\mathrm{R}$ & 54 & 6 & -14 & 60 & 56.39 \\
\hline 32 & Temporal Lobe & $\mathrm{R}$ & 40 & 32 & -58 & 12 & 54.24 \\
\hline 33 & $\begin{array}{l}\text { Superior } \\
\text { Temporal Gyrus }\end{array}$ & $\mathrm{R}$ & 38 & 52 & -32 & 6 & 56.25 \\
\hline 34 & $\begin{array}{l}\text { Cerebellum } \\
\text { Posterior Lobe }\end{array}$ & L & 36 & -16 & -76 & -24 & 55.87 \\
\hline 35 & $\begin{array}{l}\text { Parietal } \\
\text { Lobe - Precuneus }\end{array}$ & L & 27 & -2 & -38 & 48 & 55.15 \\
\hline 36 & $\begin{array}{l}\text { Cerebellum } \\
\text { Posterior Lobe }\end{array}$ & $\mathrm{R}$ & 24 & 32 & -68 & -28 & 54.18 \\
\hline 37 & $\begin{array}{l}\text { Frontal Gyrus } \\
\text { (/ White matter) }\end{array}$ & L & 22 & -18 & -10 & 36 & 55.89 \\
\hline 38 & $\begin{array}{l}\text { Middle Frontal } \\
\text { Gyrus - Supp. } \\
\text { Motor Area }\end{array}$ & L & 19 & -14 & -8 & 64 & 54.73 \\
\hline 39 & $\begin{array}{l}\text { Posterior } \\
\text { Cingulate }\end{array}$ & $\mathrm{R}$ & 17 & 8 & -64 & 10 & 54.62 \\
\hline 40 & $\begin{array}{l}\text { Middle Temporal } \\
\text { Gyrus }\end{array}$ & L & 14 & -56 & -28 & -18 & 54.06 \\
\hline 41 & $\begin{array}{l}\text { Cerebellum } \\
\text { Anterior Lobe }\end{array}$ & $\mathrm{R}$ & 14 & 2 & -60 & -12 & 53.19 \\
\hline 42 & Supp. Motor Area & L & 13 & -10 & 0 & 70 & 54.82 \\
\hline 43 & $\begin{array}{l}\text { Middle Temporal } \\
\text { Gyrus }\end{array}$ & L & 12 & -50 & -64 & 28 & 55.57 \\
\hline 44 & $\begin{array}{l}\text { Precuneus - } \\
\text { Posterior } \\
\text { Cingulate }\end{array}$ & L & 12 & -12 & -42 & 44 & 54.54 \\
\hline 45 & $\begin{array}{l}\text { Superior Parietal } \\
\text { Lobule / Angular } \\
\text { Gyrus }\end{array}$ & $\mathrm{R}$ & 11 & 36 & -66 & 44 & 54.15 \\
\hline
\end{tabular}


Supplementary Table 2. (Continued)

\begin{tabular}{|c|c|c|c|c|c|c|c|}
\hline \multirow[t]{2}{*}{ Cluster } & \multirow[t]{2}{*}{$\begin{array}{l}\text { Anatomical } \\
\text { region }\end{array}$} & \multirow[t]{2}{*}{ Hemisphere } & \multirow{2}{*}{$\begin{array}{l}\text { Clustersize } \\
\text { (num. of } \\
\text { voxels) }\end{array}$} & \multicolumn{3}{|c|}{$\begin{array}{l}\text { peak MNI } \\
\text { coordinates }\end{array}$} & \multirow{2}{*}{$\begin{array}{c}\text { Percentage } \\
\text { Accuracy }\end{array}$} \\
\hline & & & & $x(\mathrm{~mm})$ & $y(m m)$ & $\mathrm{z}(\mathrm{mm})$ & \\
\hline 46 & Temporal Lobe & $L$ & 10 & -40 & -50 & -10 & 55.56 \\
\hline 47 & $\begin{array}{l}\text { Inferior Parietal } \\
\text { Lobule }\end{array}$ & L & 9 & -42 & -40 & 22 & 55.25 \\
\hline 48 & $\begin{array}{l}\text { White Matter and } \\
\text { Insula ( } 2 \text { vox.) }\end{array}$ & L & 8 & 28 & 16 & 10 & 53.53 \\
\hline 49 & Parietal Lobe & L & 8 & 30 & -64 & 30 & 55.67 \\
\hline 50 & Occipital Lobe & L & 8 & -22 & -82 & 32 & 57.30 \\
\hline 51 & $\begin{array}{l}\text { Medial Frontal } \\
\text { Gyrus - Paracen- } \\
\text { tral Lobule }\end{array}$ & L & 8 & -4 & -22 & 72 & 55.84 \\
\hline 52 & $\begin{array}{l}\text { Cerebellum } \\
\text { Posterior Lobe }\end{array}$ & L & 7 & -24 & -76 & -28 & 54.01 \\
\hline 53 & Precentral Gyrus & L & 7 & -36 & -18 & 38 & 55.26 \\
\hline 54 & $\begin{array}{l}\text { Inferior Temporal } \\
\text { gyrus }\end{array}$ & $\mathrm{R}$ & 6 & 54 & -20 & -22 & 55.59 \\
\hline 55 & Parietal Lobe & $\mathrm{R}$ & 6 & 24 & -42 & 52 & 53.62 \\
\hline \multicolumn{8}{|c|}{ Neutral focus } \\
\hline 56 & $\begin{array}{l}\text { Inferior Parietal } \\
\text { Lobe }\end{array}$ & $\mathrm{R}$ & 234 & 40 & -28 & 30 & 58.49 \\
\hline 57 & $\begin{array}{l}\text { Parietal } \\
\text { Lobe - Precuneus }\end{array}$ & L & 225 & -16 & -42 & -62 & 57.17 \\
\hline 58 & Precentral Gyrus & L & 99 & -48 & -22 & 38 & 56.94 \\
\hline 59 & $\begin{array}{l}\text { Parietal } \\
\text { Lobe - Precuneus }\end{array}$ & $\mathrm{R}$ & 86 & 20 & -58 & 54 & 56.36 \\
\hline 60 & Angular Gyrus & $\mathrm{R}$ & 38 & 36 & -58 & 32 & 56.87 \\
\hline 61 & $\begin{array}{l}\text { Paracentral } \\
\text { Lobule }\end{array}$ & $\mathrm{R}$ & 29 & 18 & -38 & 56 & 55.15 \\
\hline 62 & $\begin{array}{l}\text { Parietal } \\
\text { Lobe - Precuneus }\end{array}$ & $\mathrm{R}$ & 25 & 8 & -38 & 46 & 56.41 \\
\hline 63 & Sub-Gyral & L & 15 & -20 & -16 & 54 & 55.03 \\
\hline 64 & $\begin{array}{l}\text { Sub-Gyral - incl. } \\
\text { Insula (3 vox.) }\end{array}$ & L & 14 & -40 & 10 & 20 & 55.31 \\
\hline 65 & Parietal Lobe & L & 12 & -38 & -36 & 20 & 54.94 \\
\hline
\end{tabular}




\begin{tabular}{llllllll}
\hline Cluster & $\begin{array}{l}\text { Anatomical } \\
\text { region }\end{array}$ & Hemisphere $\begin{array}{c}\text { Clustersize } \\
\text { (num. of } \\
\text { voxels) }\end{array}$ & $\begin{array}{c}\text { peak MNI } \\
\text { coordinates } \\
\mathbf{x}(\mathbf{m m})\end{array}$ & $\begin{array}{c}\text { y(mm) } \\
\mathbf{z}(\mathbf{m m})\end{array}$ \\
\hline $\mathbf{6 6}$ & $\begin{array}{l}\text { Posterior } \\
\text { Cingulate }\end{array}$ & $\mathrm{R}$ & 11 & 20 & -66 & 8 & 54.75 \\
& $\mathbf{6 7}$ & $\mathrm{L}$ & 7 & -32 & -88 & -8 & 55.47 \\
$\mathbf{6 8}$ & Occipital Lobe & $\mathrm{L}$ & 7 & -16 & -82 & -8 & 54.51 \\
$\mathbf{6 9}$ & Occipital Lobe & $\mathrm{L}$ & 7 & 18 & -78 & -6 & 56.20 \\
$\mathbf{7 0}$ & Occipital Lobe & $\mathrm{R}$ & 7 & -36 & -10 & 54.94 \\
& Middle Temporal & $\mathrm{R}$ & 7 & 56 & -36 & & \\
\hline
\end{tabular}

Abbreviations: $\mathrm{L}=$ left. $\mathrm{R}=$ right. $\mathrm{B}=$ Bilateral. $\mathrm{MNI}=$ Montreal Neurological Institute. 
Supplementary Table 3. Food stimuli selection ratings per participant. The averages of fives selected unpalatable of palatable foods, their standard deviations and ranges are presented per participant.

\begin{tabular}{|c|c|c|c|c|}
\hline \multirow[t]{2}{*}{ Participant } & \multicolumn{2}{|c|}{ Unpalatable foods } & \multicolumn{2}{|c|}{ Palatable foods } \\
\hline & Average (SD) & Range & Average (SD) & Range \\
\hline 1 & $2.40(1.34)$ & $1-4$ & $9.20(1.10)$ & $8-10$ \\
\hline 2 & $2.00(1.00)$ & $1-3$ & $9.40(0.55)$ & $9-10$ \\
\hline 3 & $2.00(1.41)$ & $1-4$ & $9.60(0.89)$ & $8-10$ \\
\hline 4 & $2.00(0.00)$ & $2-2$ & $8.80(1.10)$ & $8-10$ \\
\hline 5 & $2.60(1.52)$ & $1-4$ & $10.00(0.00)$ & $10-10$ \\
\hline 6 & $1.80(0.84)$ & $1-3$ & $9.20(0.45)$ & $9-10$ \\
\hline 7 & $1.80(0.45)$ & $1-2$ & $8.80(0.45)$ & $8-9$ \\
\hline 8 & $1.00(0.00)$ & $1-1$ & $9.00(1.00)$ & $8-10$ \\
\hline 9 & $2.40(1.34)$ & $1-4$ & $9.00(0.71)$ & $8-10$ \\
\hline 10 & $1.60(0.89)$ & $1-3$ & $9.00(1.00)$ & $8-10$ \\
\hline 11 & $2.60(2.07)$ & $1-6$ & $9.20(0.84)$ & $8-10$ \\
\hline 12 & $1.40(0.55)$ & $1-2$ & $9.00(0.71)$ & $8-10$ \\
\hline 13 & $2.00(1.41)$ & $1-4$ & $9.20(1.10)$ & $8-10$ \\
\hline 14 & $3.20(2.05)$ & $1-5$ & $9.00(0.00)$ & $9-9$ \\
\hline 15 & $3.60(2.79)$ & $1-7$ & $9.00(1.00)$ & $8-10$ \\
\hline 16 & $7.00(1.41)$ & $5-9$ & $10.00(0.00)$ & $10-10$ \\
\hline 17 & $1.80(1.30)$ & $1-4$ & $10.00(0.00)$ & $10-10$ \\
\hline 18 & $2.20(0.84)$ & $1-3$ & $9.00(0.71)$ & $8-10$ \\
\hline 19 & $5.00(0.71)$ & $4-6$ & $8.60(1.34)$ & $7-10$ \\
\hline 20 & $1.40(0.89)$ & $1-3$ & $10.00(0.00)$ & $10-10$ \\
\hline 21 & $1.80(0.84)$ & $1-3$ & $10.00(0.00)$ & $10-10$ \\
\hline 22 & $1.00(0.00)$ & $1-1$ & $10.00(0.00)$ & $10-10$ \\
\hline 23 & $4.80(0.45)$ & $4-5$ & $8.40(2.30)$ & $5-10$ \\
\hline Mean total & $2.50(1.42)$ & & $9.28(0.50)$ & \\
\hline
\end{tabular}

Abbreviation: SD = standard deviation 


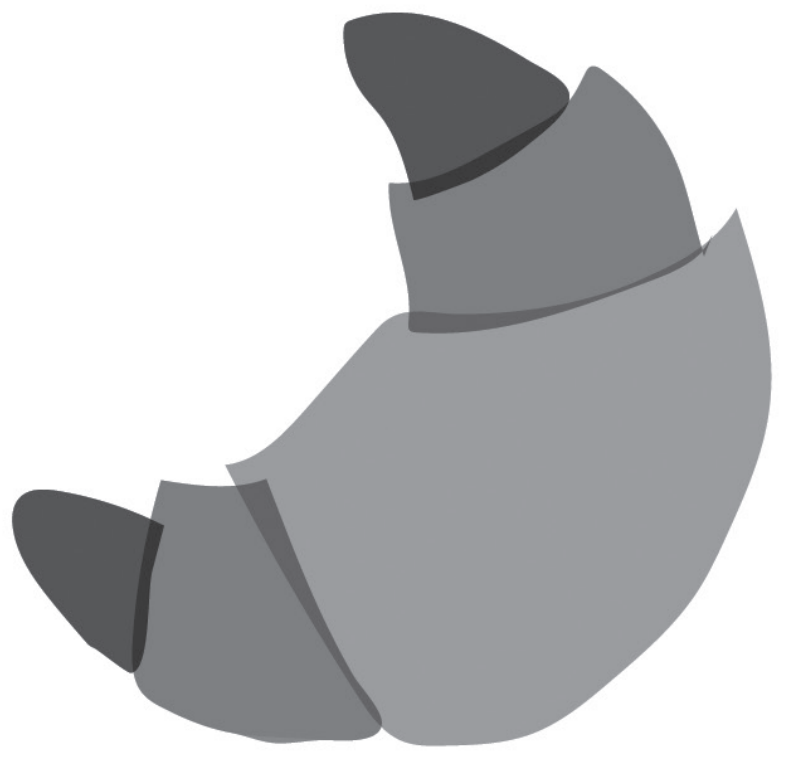




\section{CHAPTER 3}

\section{EFFECTS OF MINDSET ON HORMONAL RESPONDING. NEURAL REPRESENTATIONS, SUBJECTIVE EXPERIENCE AND INTAKE}

Submitted as: Franssen, S., Jansen, A., van den Hurk, J., Adam, T., Roebroeck, A. and Roefs, A., Effects of mindset on hormonal responding, neural representations, subjective experience and intake. Physiology and Behavior. 


\section{Abstract}

A person can alternate between food-related mindsets, which for instance depends on one's emotional state or situation. Being in a certain mindset can influence foodrelated thoughts, but interestingly it might also trigger physiological factors involved in eating. The current study investigates the influence of an induced 'loss of control' mindset as compared to an 'in control' mindset on hormonal, neural and behavioural responses to chocolate stimuli. Mindsets were induced by having chocolate lovers view a short movie during two sessions in a within-subjects design. Neural responses to visual chocolate stimuli were measured using an ultra-high field (7T) scanner. Momentary ghrelin and GLP-1 levels were determined on five moments and were simultaneously assessed with self-reports on perceptions of chocolate craving, hunger and feelings of control. Furthermore, chocolate intake was measured using a bogus chocolate taste test. It was hypothesized that the loss of control mindset would lead to hormonal, neural and behavioural responses that prepare for ongoing food intake, even after eating, while the control mindset would lead to responses reflecting satiety. Results show that neural activity in the mesocorticolimbic system is stronger for chocolate stimuli than for neutral stimuli and that ghrelin and GLP-1 levels responds to food intake, but that these effects are not moderated by mindset. Self-reported craving and actual chocolate intake are affected by mindset, in that cravings and intake are higher with a loss of control mindset than with a control mindset. These findings indicate that subjective experience and behaviour are affected, whereas hormonal and neural responses are not influenced by mindset.

\section{Keywords}

Eating behaviour, fMRI, mesocorticolimbic system, ghrelin, glp-1, mindset, obesity 


\section{Introduction}

In today's society, more than 1.9 billion adult people are estimated to be overweight or obese (World Health Organization, 2020). The major cause of overweight is a prolonged energy imbalance, with the number of consumed calories exceeding the number of expended calories (Hall et al., 2012). Though overweight people frequently attempt to lose weight, the number of successful weight loss maintainers is low (Fildes et al., 2015). Increased responding to food cues or contexts that signal the availability of tasty foods potentially sabotages healthy eating in dieters and weight loss maintainers (Schyns et al., 2020). This increased responding to food cues or food contexts includes increased food cravings, hunger related hormonal responses and reward-related neural activation; the so-called food cue reactivity prepares for intake and easily leads to overeating (Boswell \& Kober, 2016; Jansen, 1998; Jansen et al., 2003, 2016). Food cue reactivity is significantly stronger in obese people compared to healthy weight people (Boswell \& Kober, 2016; Boutelle \& Bouton, 2015; Ferriday \& Brunstrom, 2011; Jansen et al., 2003; Tetley et al., 2009; van den Akker, Stewart, et al., 2014), and variance in food cue reactivity was found to account for $26 \%$ of the variance in eating and weight gain (Boswell \& Kober, 2016). The increased responding to food cues and contexts has been associated with specific mindsets (see e.g., Jansen et al., 2016; Siep et al., 2012). It has, for example, been demonstrated decades ago that beliefs about caloric load, can influence eating behaviour (see e.g., Wooley, 1972). More recent studies investigating the effects of health claims or caloric content labels on high caloric food consumption support the early findings: food intake is increased when a label indicates that the food is low calorie or healthy while intake is decreased by a high caloric content label or unhealthy label (e.g., McCann et al., 2013; Roefs \& Jansen, 2004; Shide \& Rolls, 1995). Likewise, control and loss of control mindsets are associated with the inhibition and disinhibition of food intake (Schyns et al., 2020). A mindset is a set of thoughts, beliefs, assumptions and expectations that someone has about a certain topic. Mindsets are assumed to be dynamic, meaning that a person can alternate between mindsets, depending on for example one's emotional state or the situation (Roefs et al., 2018). Overweight people and unsuccessful dieters often report a 'loss of control' mindset; they frequently believe that they are unable to stop or prevent (over)eating when triggered by specific cues. Though the effects of labels on food intake have been studied a number of times, the effects of clinically relevant control versus loss of control mindsets have never been manipulated in experimental 
studies. Therefore, the current study aims to investigate whether and how an induced 'loss of control' mindset as compared to an 'in control' mindset affects neural representations of visual chocolate stimuli, hormonal responses to the anticipation and consumption of chocolate, subjective experiences and the amount of chocolate consumed.

It has been suggested that effects of mindset on eating behavior are mediated via physiological mechanisms, for example via hormonal signaling (Cassady et al., 2012; Crum et al., 2011). Gut hormones interact with the central nervous system, which is also referred to as the gut-brain axis (Perello \& Dickson, 2014; Schwartz et al., 2000; Sun et al., 2014). One of those hormones is ghrelin, also considered the 'hunger hormone', which is released from endocrine cells of the stomach (Kojima et al., 1999) and binds with the brains' receptors in the arcuate nucleus and ventromedial hypothalamus to regulate appetitive motivation (Baynes et al., 2006; Morton et al., 2006; Murphy \& Bloom, 2006; Rhea et al., 2018). Ghrelin is regulated by energy and nutrient sensory processing of the gastrointestinal tract; when energy intake is low or the stomach is empty, ghrelin is released (Baynes et al., 2006; Murphy et al., 2006). Ghrelin levels were affected by expectations of low versus high caloric content as suggested by a product label, while in reality there was no caloric difference between these drinks (Crum et al., 2011). More specifically, ghrelin concentrations rose from baseline to viewing the label (just before consumption) and dropped after consumption with the high-caloric label only, whereas the ghrelin response was flat with the low-caloric label. Another example of an endocrine hormone involved in appetitive motivation is glucagon-like peptide 1 (GLP-1). GLP-1 is secreted from the small intestine and colon into circulation stimulating insulin release from the pancreas, and inhibiting glucagon release (Drucker, 2006). GLP-1 producing cells are also located in the nucleus of the solitary tract with projections throughout the brain (Schwartz et al., 2000; Vrang \& Larsen, 2010) and it is referred to one of the gut 'satiety hormones' (Dailey \& Moran, 2013; Flint et al., 1998). It was found that GLP-1 secretion increased or decreased by participants beliefs after drinking a more or less satiating preload, whereas the preload nutritional content was kept exactly the same (Cassady et al., 2012). Moreover, increasing the GLP-1 receptor activation led to an increased perception of satiety and decrease of anticipated neural reward activity (van Bloemendaal et al., 2015), indicating the bidirectional nature of the gut-brain interaction. Generally, these studies suggest that cognitive modulations can affect hormonal processes involved in appetitive motivation. 
While peripheral hormones interact with regulatory brain areas, prompting increase or decrease in hunger feelings (Morton et al., 2006; Murphy et al., 2006), eating behaviour is not solely a result of homeostatic hunger, but also a result of hedonic hunger related to the rewarding value of food (Berridge et al., 2010; D’Agostino \& Small, 2012; Lowe \& Butryn, 2007). To shed light on the neural response involved in processing the rewarding value of food, many functional magnetic resonance imaging (fMRI) studies have been conducted (e.g., see the review of van der Laan et al., (2011). However, the results on the rewarding value of food from studies with healthy-weight people (see van der Laan, de Ridder, Viergever, \& Smeets, 2011) and studies comparing healthy-weight to people who are overweight (see e.g., Ziauddeen, Farooqi, \& Fletcher, 2012) have been rather inconsistent. A reason for this inconsistency might be that mindset was not considered in these studies. That is, these studies often employed a so-called passive viewing paradigm, in which participants view food stimuli without specific instructions (e.g., Martin et al., 2010; Rothemund et al., 2007; Stoeckel et al., 2008; Thomas et al., 2015). Importantly, in a passive viewing paradigm, it is unclear what the ongoing mental process of the participant is. While the researcher may assume that the participant is evaluating the tastiness or reward value of the food, the focus may just as well be on the healthiness or caloric content of the presented foods. Without a clear mental task, a researcher can only base conclusions on reverse inference (Poldrack, 2011). That is, drawing conclusions about the engaged mental processes based on the observed locations of neural activity. To understand the neural reward system in a more complete way, a researcher must aim for a clear idea on how participants are evaluating/processing the presented food stimuli, which may be affected by the current mindset (Roefs et al., 2018).

Some fMRI studies have previously addressed how mindsets and task demands affect neural responses to food stimuli (Bhanji \& Beer, 2012; Frankort et al., 2012; Hare et al., 2011; Hege et al., 2018; Schroder et al., 2014; Siep et al., 2012). More specifically, it has been shown that neural responses in the mesocorticolimbic system were greater in overweight people than in healthy-weight people when participants were required to focus on the tastiness of stimuli, but not when they were not given any instructions on how to evaluate the presented foods (Frankort et al., 2012). Moreover, neural activity has been shown to be decreased by suppressing food craving relatedthoughts while viewing high caloric food stimuli (Siep et al., 2012). One of our recent 
studies likewise showed that neural activity in the mesocorticolimbic system was greater when the task demands required participants to evaluate the tastiness of the presented foods as compared to when they were required to evaluate the colors of the exact same foods in females who are overweight (Franssen et al., 2020). Another study showed that neural taste and pleasure experiences can be strongly influenced by people's beliefs, as induced by label contents. Neural activity in the orbitofrontal cortex (OFC) while tasting wine, was purely influenced by manipulating wine price labels. While presenting the same wine stimuli, higher priced wines were rated as more pleasurable and the perceived pleasure correlated with increased activity in the OFC as compared to lower priced wines (Plassmann et al., 2008). Likewise, brand labels presented with cola stimuli have also been found to affect taste ratings and neural reward signaling as represented in medial OFC, amygdala and striatum (Kühn \& Gallinat, 2013). So, the dynamic nature of food-reward strongly depends on task demands, attentional focus and someone's current mindset (Roefs et al., 2018).

There is a complicating factor in this line of research. While it is often assumed that neural activity in response to high caloric food stimuli in the mesocorticolimbic system is proportionate to the reward value of the presented stimuli, this is not at all straightforward. Instead, it has been proposed that activity in the mesocorticolimbic system reflects motivational saliency (Salamone \& Correa, 2012). In support of this idea, it has been found that both rewarding and aversive stimuli led to neural activity in largely overlapping brain regions (Chikazoe et al., 2014). Moreover, in a previous study of our lab, we observed no significant differences in the level of neural activity in the mesocorticolimbic system between highly palatable high caloric and highly unpalatable high caloric food stimuli (Franssen et al., 2020). Taken together, these findings suggest that activity in mesocorticolimbic brain areas reflect motivational saliency (either negative or positive), as opposed to reward value.

This raises the question how the brain codes the reward value if it is not the average level of activity in certain brain regions. It has been suggested that reward value might be reflected in so-called multivoxel patterns of neural activity (Kahnt, 2018). A recent study showed that food value can be clearly decoded from multivoxel patterns of neural activity in both the lateral and medial OFC (Suzuki et al., 2017). Dovetailing nicely with these findings, in a previous study from our lab, we could only decode food palatability from multivoxel patterns of neural activity, whereas we did not find significant food palatability differences using mass-univariate analyses 
(Franssen et al., 2020). Therefore, it is important to complement the standard massunivariate analyses with Multi Voxel Pattern Analysis (MVPA), to better understand how the brain codes reward value, and how it might be affected by current mindset.

To examine the influence of mindset on physiological mechanisms involved in appetite motivation, the current study measured the effects of a manipulated control and loss of control mindset in two separate sessions on hormonal, neural and behavioural responses to chocolate stimuli. In a within-subjects design, a loss of control and a control mindset were induced by having chocolate lovers view a short movie. Subsequently, neural responses to visual chocolate stimuli were measured using fMRI using a state-of-the-art ultra-high field (7T) scanner. Brain regions expected to be involved for chocolate craving were ventral striatum with nucleus accumbens (NAcc), midbrain and the OFC, whereas for control the inhibitory-control-related brain regions include: dorso- and ventro-lateral prefrontal cortex (dIPFC, VIPFC), parietal posterior cortex (PPC), dorsal anterior cingulate cortex (dACC), caudate, pre supplementary motor area (preSMA) and the globus pallidus (GP) (Giuliani et al., 2018). To determine momentary active GLP-1 and ghrelin levels, blood samples were taken on 5 pre and post mindset induction moments and were simultaneously assessed with self-reports on perceptions of chocolate craving, hunger and feelings of control. Furthermore, chocolate intake was measured after the mindset manipulation using a bogus chocolate taste test. It was hypothesized that the manipulated loss of control mindset would lead to hormonal and neural responses that prepare for ongoing food intake, even after eating, while the manipulated control mindset would lead to hormonal and neural responses reflecting satiety. More specifically, a loss of control mindset - as compared to the in control mindset - was expected to lead to increased self-reported cravings and hunger, decreased feelings of control, increased chocolate consumption, increased ghrelin levels, and decreased GLP-1 levels. For the level of neural activity (mass-univariate analyses), we expected an increased activity in craving-related brain areas to chocolate versus neutral images in the loss of control mindset, whereas, more control-related activity was expected to chocolate stimuli in the control mindset. For multivariate analyses (using MVPA), differences were expected between the two mindsets in distinguishing neural representations of chocolate versus neutral images and predominantly in food-craving brain areas when in a loss of control mindset. 


\section{Material and methods}

\section{Participants}

Twenty-six Dutch female undergraduates were recruited by advertisements at Maastricht University. Inclusion criteria included: right-handiness, a female gender, a healthy weight (18.5 < Body Mass Index (BMI) < 25), liking of milk and dark chocolate (scoring on average $\geq 70$ out of 100), having no MRI contra-indications and no history of neurological or psychological illnesses. This study was approved by the Medical Research Ethics Committee of Maastricht University Medical Centre. After completion, participants received $€ 100$ as compensation for their time.

\section{Cognitive and Behavioural Assessments}

\section{The Restraint Scale}

Participants filled out the Restraint Scale (Herman \& Polivy, 1980), which measures the intention to restrict food intake, concerns about body weight, and weight fluctuations. It consists of 10 questions that are scored on a scale from 0 to 3 or 4 each. The minimum total score is 0 , and the maximum is 35 , with higher scores reflecting higher intentions to restrain intake. The internal consistency in the current sample was high, with a Cronbach's alpha of 0.851 .

\section{Chocolate liking}

As part of the screening procedure, chocolate liking was measured with two questions on 100 mm Visual Analogue Scales (VAS) as part of the screening procedure: "How much do you like milk chocolate?" and "How much do you like dark chocolate?" ranging from 0 "not at all" to 100 "very much". The average of the two rating outcomes was calculated.

\section{Chocolate craving questionnaire}

To assess participants' trait chocolate craving, the Craving part (Factor 1) from the Attitudes to Chocolate Questionnaire (ACQ) (Benton et al. 1998) was administered at the end of the second session. This questionnaire consists of 17 items, individually scored on a Likert scale from -3 till 3 with a minimum score of -51 and maximum of 51. The internal consistency in the current sample was high, with a Cronbach's alpha of 0.868 . 


\section{Momentary self-reports}

State chocolate cravings, hunger, and feelings of control over eating chocolate were assessed using $100 \mathrm{~mm}$ Visual Analogue Scales (VAS), ranging from 0 (not at all/totally disagree) to 100 (very much/totally agree). The questions included: "How much do you crave chocolate at this moment?", "How hungry do you feel at this moment?", and were rated on the not at all - very much scale and the statement: "At this moment I would be able to control myself and not eat chocolate" was rated on the disagree - agree scale. The VASs were administered with paper and pencil, except for the one that was administered after the mindset manipulation in the scanner, for which a screen and joystick were used. Note that this digital measurement directly after manipulation could also be viewed as a manipulationcheck.

\section{Bogus chocolate taste test}

Ad libitum chocolate intake was measured in a bogus taste test. During this taste test, participants rated milk chocolate and dark chocolate on taste, texture and liking using 0-100 mm VASs. The milk and dark chocolate were each presented in two cups with 20 grams each, so that participants were in total presented with 80 grams of chocolate (in total $437 \mathrm{kcal}, 40$ grams milk: $221 \mathrm{kcal}$ and 40 grams dark: $216 \mathrm{kcal}$ ). The cups were labelled as A, B, C, and D, and presented as being slightly different. Participants were instructed to taste and rate the chocolate and it was announced that they could taste as much as they wanted. They were left alone during filling in the bogus taste test questionnaire. The bogus taste test had a set time of seven minutes, timed by the experimenter. After the taste test, the remaining chocolate was taken from the experimental room and weighed, to be able to compute the number of consumed calories. In the meantime, the participant filled in a questionnaire about her memory of the taste of the presented chocolates. This in-between questionnaire was administered to distract the participant during chocolate weighing and to maintain the cover story.

Because it was necessary for the measurement of ghrelin and GLP-1 that each participant had eaten an equal number of calories, the experimenter asked the participant to eat the remaining chocolate as well after the taste test. This requirement was disguised as a lottery, because otherwise participants would know that they would be asked to consume all chocolate in the second session as well. In this 'lottery', participants could draw a ticket, supposedly indicating 'yes, eat all chocolate', or 'no, do not eat anymore chocolate', whereas only 'yes'-tickets were included. All participants ate the remaining chocolate after the taste test in both sessions. 


\section{Experimental procedure}

First, participants were screened for eligibility by filling in an online screeningquestionnaire and when meeting the inclusion criteria, participants were scheduled for the two experimental sessions. In each session, one of the two mindsets was induced: (1) a loss of control mindset or (2) a control mindset. Order of conditions was counterbalanced across participants, so that $50 \%$ had first the loss of control mindset and $50 \%$ the control mindset. As the phase of the menstrual cycle may influence feelings of craving or neural responses to food (Dreher et al., 2007), the two sessions took place at approximately the time of the menstrual cycle ${ }^{3}$. All sessions took place at the same time of the day (between 10:00 -12:00 h AM). Participants were asked to arrive at the lab fasted (since bedtime, except for water), to control for hunger state differences and to assess baseline hormone levels from blood samples. Moreover, participants were asked to refrain from alcohol 24 hours before each session started.

Participants were informed about the whole experimental procedure and gave their informed consent. Then an intravenous canula was placed in an antecubital vein, and a fasted baseline blood sample (T1) was obtained. After 15 minutes, participants received a standardized low calorie breakfast (approx. $150 \mathrm{kcal}$ ), which consisted of a wholegrain cracker with cheese $e^{4}$ and a glass of orange juice (150 ml). After breakfast, the scanning procedure was explained. Fifteen minutes after breakfast, the second blood sample (T2) was obtained, which was followed by the one-hour scan-session. After the anatomical scan, the mindset induction took place in the scanner and directly afterwards, the third blood sample (T3) was taken. Then the acquisition of the 4 functional runs started. After the scan-session, the fourth blood sample (T4) was obtained and ad libitum chocolate intake was measured in a bogus chocolate taste test (see paragraph "Bogus chocolate taste test" below). Fifteen minutes after intake, the fifth blood sample (T5) was obtained. At each blood sampling time-point, participants indicated their chocolate craving, hunger and feelings of control on Visual Analogue Scales (VAS) (see Figure 1 for timeline). At the end of the second session, height and weight were measured to calculate BMI.

\footnotetext{
${ }^{3}$ One participant had 2 months between 2 sessions, because of technical issues with the scanner during the 2 nd appointment.

${ }^{4}$ One participant had chicken breast filet instead of cheese, however the total calories consumed was the same
} 


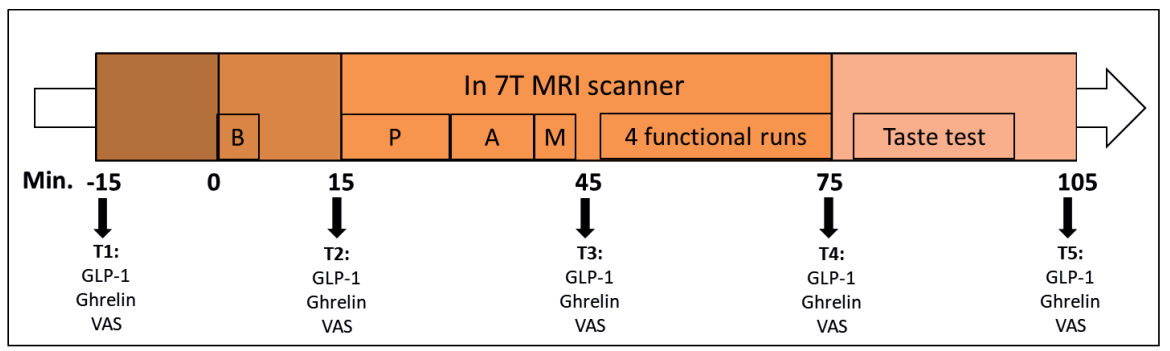

Figure 1: Experimental procedure with timeline and timepoints of blood sampling with self-reports. Abbreviations: $B=$ standardized breakfast, $P=$ positioning \& preparation scanning, $\mathrm{A}=$ anatomical scan, $\mathrm{M}=$ mindset manipulation, $\mathrm{T}=$ time point, $\mathrm{VAS}=$ visual analogue scales questionnaires.

\section{Hormones}

Blood samples were intravenously collected from an antecubital vein in ice-cooled, EDTA-coated tubes, with a total amount of 100 ccs (50 ccs per session) to determine active ghrelin and GLP-1 levels. Blood samples were directly centrifuged at $4^{\circ} \mathrm{C}$, 3000 rpm for $10 \mathrm{~min}$. and plasma was kept in a nitrogen bucket $\left( \pm-200{ }^{\circ} \mathrm{C}\right)$. Next, plasma was in duplo stored at $-80^{\circ} \mathrm{C}$ until analysis. In the EDTA-coated tubes for GLP1 analysis levels, the protease inhibitors dipeptidyl peptidase-IV (DPP-IV) (Millipore) was added to prevent degradation. Plasma for ghrelin determination was treated with methanol $(\mathrm{HCL})$ and protease inhibitor phenyl-methyl-sulfonyl fluoride (PMSF) to prevent degradation. Ghrelin levels were measured using a double antibody radioimmunoassay (RIA) with an intra-assay variability of 15\%. The lowest detectable active ghrelin was $26.11 \mathrm{pg} / \mathrm{ml}$. Active plasma GLP-1 was determined by JJ Laboratory Services of University College London (UCL) with an assay sensitivity of $0.14 \mathrm{pmol} / \mathrm{L}$, and with an intra-assay variability of $3-6 \%$ and an inter-assay variability of $10-13 \%$ by using commercial ELISAs (Cat. EZGLP-35K, Millipore).

\section{Mindset manipulation}

A mindset was induced using a short movie ( $\pm 75 \mathrm{sec}$ ), which participants viewed while in the MRI scanner prior to the acquisition of the functional runs. The movies consisted of pictures with words and short sentences that were related either to 'loss of control' or to 'control' (see below for details). Participants were instructed to vividly imagine being in the situation as illustrated in the movie and to maintain the induced feelings as long as possible. After seeing the short movie, participants were asked to eat a small piece of chocolate (approx. 4 gr), to mimic the real-life situation with a control 
or loss of control feeling about eating something palatable and high caloric. To keep the mindset active during the functional scanning protocol, participants were primed eight times for three seconds during the fMRI task with 8 movie stills. The priming pictures were accompanied with the visual instruction: "Try to vividly imagine as much as possible being in the situation as previously shown in the movie". The mindset induction movies (control and loss of control) were first tested in a between-subjects design including twenty participants ( $n=10$ for each condition) not taking part in the actual study. The results of this pilot study showed that the mindset induction movies had the intended effects: State craving was higher after the loss of control mindset induction $(M=69.50, S D=14.58)$ than after the control mindset induction ( $M$ $=43.10, S D=28.84, t(13.32)=2.58, p<0.05)$. Also, feelings of control (I want to control myself: $t(11.84)=5.53, p<0.001$, and I can control myself: $t(18)=3.16, p=0.005$ ) were stronger after the control mindset induction (want control: $M=80.00, S D=7.87$; can control: $M=80.70, S D=1.57$ ) than after the loss of control mindset induction (want control: $M=45.10, S D=9.56$; can control: $M=65.40 S D=11.06$ ).

\section{Loss of control mindset}

For inducing a loss of control mindset, pictures related to a hedonic lifestyle, foods, and enjoyable (social) situations were shown together with words/short sentences to further emphasize the intended mindset. Example pictures included: eating pizza with a group of people and a person relaxing on the beach with a nice cocktail. Example words/short sentences included: 'enjoy', 'holiday', and 'let yourself go'.

\section{Control mindset}

For the control mindset, pictures consisted of healthy food items or healthy behaviour and being in control (social) situations. These pictures were also shown together with words/short sentences to further emphasize the intended mindset. Example pictures included: eating a healthy salad and a person standing on a scale. Example words/ short sentences included: 'health', 'in control', and 'conscious'.

\section{Manipulation Check}

To check the perception of the induced mindset 'control' or 'loss of control', the participant was asked at the end of each session to what extent she could vividly imagine the depicted situations, and to what extent she could hold on to that feeling after seeing the mindset movie. Questions on feelings of being in control and 
chocolate craving after seeing the movie were also measured retrospectively at the end of each session using 100 mm VAS scales ranging from 0 "not at all" or to 100 "very much". In addition, participants were asked to describe what they thought the aim of the study was to check if their ideas could have biased the results.

\section{Visual stimuli presented during scanning}

Stimuli included 40 pictures of chocolate (dark and milk) products and 40 neutral pictures of office supplies. Neutral pictures were not related to food and were matched as closely as possible on size to the chocolate pictures. Each picture was presented once per run and in total four times during the scanning session. Chocolate pictures did not have any festive associations (e.g., Valentine's Day or Christmas). All stimuli were presented as pop-out figures on a light grey background (RGB: 191191 191; CKYM: 252020 0) in the center of a black screen, covering a visual angle of approximately $12^{\circ}$.

\section{Stimulation protocol fMRI}

The stimuli were presented in a blocked design using E-prime (version 2.0, Psychology Software Tools Inc., Pittsburg, PA, USA), with 2 blocks of interest (chocolate and neutral stimuli) and 1 block of no interest (question + mindset priming). Each block of interest lasted 16 s, and included either 8 chocolate or 8 neutral pictures. Each picture was selected randomly without replacement from the picture pool (until pool was empty), and presented for 2000 ms. To maintain the participant's attention and to keep the mindset manipulation salient, two of the stimulus blocks were followed by a question + mindset priming block (3500 ms question, 6500 ms mindset priming). These question and mindset priming blocks were randomly placed in each run, with one occurring after a chocolate block and one after a neutral block. During these blocks, first a picture was presented, and the participant was asked to indicate if that picture was also presented during the preceding stimulus block, by using a joystick. Next, the mindset priming was presented.

A fixation cross was presented before each block (12 s for a stimulus block and $6 \mathrm{~s}$ for a question + mindset priming block) and after the last block (12 s). So, in total each run consisted of 5 chocolate blocks, 5 neutral blocks, and 2 question + mindset priming blocks. Each scanning session consisted of 4 runs, which in total lasted approximately 30 minutes. Prior to the functional runs, an anatomical scan ( \pm 10 minutes) was acquired. 


\section{MRI acquisition}

All images were acquired on a $7 T$ Siemens MRI scanner, equipped with $70 \mathrm{mT} / \mathrm{m}$, $200 \mathrm{~T} / \mathrm{m} / \mathrm{s}$ whole body gradient coil and a 32 channel RF-coil. T T $_{1}$ weighted anatomical images and quantitative $T_{1}$ maps were acquired using an MP2RAGE sequence with the following parameters: TR $=5000 \mathrm{~ms}$, TE $=2.47 \mathrm{~ms}, \mathrm{TI} 1=900 \mathrm{~ms}$ TI $2=2750 \mathrm{~ms}$, flip angles $=5^{\circ}$ and $3^{\circ}, \mathrm{FOV}=224 \times 196 \mathrm{~mm}$ and voxel size $0.7 \times 0.7 \times 0.7 \mathrm{~mm}$. Functional $\left(\mathrm{T}_{2}{ }^{*}\right.$-weighted) images were acquired using a multiband gradient echo-planar imaging sequence (multiband factor 2, GRAPPA 3) with the Center for Magnetic Resonance Research (CMRR) multi-band C2P package (Moeller et al., 2010; Setsompop et al., 2012; Xu et al., 2013), with the following parameters: TR $=2000 \mathrm{~ms}$, TE $=18 \mathrm{~ms}$, flip angle $=75^{\circ}, F O V=200 \times 200 \mathrm{~mm}$, and voxel size of $1.25 \times 1.25 \times 1.25 \mathrm{~mm} .92$ axial slices were acquired in a backward tilted direction to the transversal - coronal line and covered the whole brain. Each participant underwent four experimental functional runs, with 172 volumes acquired per run. Thereafter, 5 functional images were acquired with opposite (anterior - posterior) phase encoding direction, for correcting magnetic susceptibility-induced distortions.

\section{Preprocessing}

Anatomical and functional data were preprocessed using BrainVoyager 20 (Brain Innovation, Maastricht, The Netherlands). Anatomical images were resampled to 1 $\times 1 \times 1 \mathrm{~mm}$ resolution, followed by brain extraction, inhomogeneity correction and transformation to MNI space.

Functional data were corrected for slice scan time differences, and subjected to motion correction using 3 rotation and 3 translation axes. Low frequency drifts in the time course were removed using a temporal high pass filter, filtering out spatial frequencies below 3 cycles per run. Then, for each run a map of estimated magnetic susceptibility-induced distortions was estimated using the target volume and the volume with opposite phase encoding direction. This map was then used to correct all functional images for geometric distortions caused by non-zero off-resonance fields. Afterwards, data were spatially smoothed by using a 2 mm FWHM Gaussian kernel. After pre-processing, functional data were co-registered to the anatomical data and transformed to 4D MNI space.

As some participants' motion resulted in significant spikes in their time courses, we submitted all functional data to a custom script that searched to identify 
and replace affected volumes. A volume was deemed affected when $2.5 \%$ or more brain voxels showed fluctuations in their time course of 4 standard deviations above or below mean signal. These volumes were replaced by a new volume, created by means of spline interpolation between the two adjacent volumes.

\section{Cognitive, behavioural and hormone analyses}

Self-reports of chocolate craving, hunger and feelings of control and blood hormone levels (ghrelin and GLP-1) were analyzed in 2 (condition: Loss of control vs Control) x 5 (time: T1 - T5) mixed ANOVAs. The difference in chocolate intake between both mindset conditions, as measured with the bogus taste test, was tested using a pairedsamples t-test. Also, differences in the manipulationcheck outcomes, were analysed with paired-samples $t$-tests.

\section{fMRI Analyses}

\section{Univariate analysis}

Data were first submitted to a General Linear Model (GLM) in Brainvoyager, including the two factors (mindset: control / no control, and stimulus type: chocolate / neutral). In addition, for each subject, the question/priming blocks and the six motion parameters were added as confounds. Some volumes were quite noise, which resulted in a striping artefact. We have identified these affected volumes and used this information as a confounder as well. The GLM extracted the set of beta values per participant at each voxel, which then served as input for the second-level random effects (RFX) analysis. We first tested the mindset $x$ stimulus type interaction effect, and created a whole brain map with the omnibus F-statistic assigned to each voxel. Then, we tested and created a whole brain map for the main effects of mindset and stimulus type.

\section{Multivariate analysis}

Whereas mass-univariate analyses of fMRI data are informative regarding involvement of brain areas in certain tasks, multivariate analysis of fMRI data can decode representational content in the brain (Norman et al., 2006). As this approach analyses multiple voxels at once, this could lead to a more sensitive detection of cognitive states. Therefore, we also used multi-voxel pattern analysis (MVPA) to test for differences in multivoxel representations of the two stimulus types between the two mindsets. 
First, single trial responses were estimated by fitting a GLM to each voxel's individual time course, thereby using one predictor for the trial of interest plus a second one that models all other trials simultaneously (see least squares simultaneous (LS-S) approach, (Mumford et al., 2012). Classification analyses were performed using functions of the CoSMo Multivariate Pattern Analysis (MVPA) toolbox (Oosterhof et al., 2016) in Matlab. Data partitions consisted of classes of chocolate and neutral stimuli trials within a mindset (loss of control condition or control condition). Unequal trials between training and test partitions were balanced using CoSMo MVPA built-in functions (Oosterhof et al., 2016). We conducted a whole brain searchlight analysis (Kriegeskorte et al., 2006), which is a descriptive method using a spherical 'searchlight' that centers on every voxel of the cortical volume, and gives an estimate of the presence of information in the spherical surrounding. On a single subject level, the algorithm targets all voxels individually and selects the voxels within the searchlight sphere with predefined radius of $6 \mathrm{~mm}$ surrounding the centred voxel. The algorithm then performs binary classification on the normalized single trial responses within the sphere's voxels, using a linear Support-Vector (LIBSVM, https://www.csie.ntu.edu. tw/ cjlin/libsvm/ ). We trained and tested the classifier on discriminating between the two stimulus types (chocolate versus neutral). The classifier was trained on trial data of 3 runs and was tested on the trials of the remaining run, following a leave-onerun-out cross-validation procedure, resulting in 4 repetitions.

For group analysis, only voxels that showed total overlap across participants were included for further analyses, to exclude voxels with poor group overlap due to imperfect inter-subject brain alignment (i.e. voxels at/outside the cortex border). Mean accuracies of stimulus types within mindset were non-parametrically tested against chance level (0.5) and were FDR corrected on voxel-level. All mean accuracies significantly higher than chance were included for interpretation of the results. To test whether prediction accuracies differed between the two mindsets, the loss of control versus control mindset stimuli accuracy differences were non-parametrically tested against 0 . 


\section{Results}

\section{Participant characteristics}

Twenty-six healthy-weight female bachelor students were included (age: $M=20.31$, $S D=1.52, \mathrm{BMI}: M=21.57 S D=1.78)$. All participants were right-handed, had no MRI contraindications and scored high on chocolate liking $(M=86.42, S D=9.68$, range: 68 - 100). The average score on the Restraint Scale was $M=11.88$ ( $S D=4.82$; range: 6.00 - 21.00). Overall participants had a relatively high score on the chocolate craving questionnaire (M: 9.11, SD: 13.40 with range: -20.00 - 38.00, of which 3 participants scored relatively low $<-15$ ).

\section{Manipulation check}

At the end of each session, VASs were administered to test the effectiveness of the induced mindset manipulations. Participants reported a significantly stronger feeling of control after the control mindset induction $(M=73.57, S D=16.65)$ as compared to the loss of control mindset induction $(M=51.84, S D=21.79), t(25)=5.69, p<$ $0.001, d=1.12$. Furthermore, chocolate craving was lower for the control $(M=$ $44.27, S D=19.93)$ than for the loss of control condition $(M=80.58, S D=13.91)$, $t(25)=9.14, p<0.001, d=1.79$. Participants in both conditions scored high on vividness of the experience (control condition: $M=73.44, S D=13.86$, loss of control condition: $M=71.64, S D=11.57$ ), with no significant difference between conditions, $t(24)=0.61, p=.549, d=0.12$. The same was true for the degree to which they could hold on to the experience after the induction (control condition: $M=$ 61.56, $S D=17.32$, loss of control condition: $M=58.52, S D=13.75$ ), with no significant difference between conditions either, $t(24)=0.94, p=.359, d=0.19 .{ }^{5}$ However, when comparing on subjective ratings on T3 (directly after manipulation) only chocolate craving was significantly higher after loss of control as compared to control. Here, no differences were found on subjective feelings of control or hunger were shown. This shows that we did found a difference in selfreported feelings in control retrospectively, whereas not directly after manipulation. Thus, the direct manipulation effect seems to be less strong on feelings of control, as compared to cravings.

${ }^{5}$ On vividness of the experience and the degree to which they could hold on to the experience, data of 1 participant is missing in 1 session 


\section{Cognitive and behavioural assessments}

We tested for differences in hunger, chocolate cravings and feelings of control, between mindset conditions and across time in 2 (condition) $\times 5$ (time) ANOVAs. No effects of mindset or mindset $x$ time are observed for the momentary self-reports of hunger and feelings of control, all $F<4.06$, al $p>0.057$. This marginally significant effect of $F=4.06, p=0.057$ is a main effect of mindset for hunger: hunger was marginally increased in the loss of control mindset. Main effects of time are observed for feelings of control, $F(4,18)=16.77, p<0.001, \eta_{p}^{2}=0.79$, and hunger, $F(4,18)=$ 21.92, $p<0.001, \eta_{p}^{2}=0.83$; feelings of control increase and hunger decreases over time. For chocolate craving, a main effect of time, $F(4,18)=32.38, p<0.001, \eta_{p}{ }^{2}=0.88$, and a main effect of mindset, $F(1,21)=13.72, p=0.001, \eta_{p}{ }^{2}=0.39$, are qualified by a marginally significant mindset $x$ time interaction, $F(4,18)=2.44, p=0.084, \eta_{p}{ }^{2}=0.35$. Chocolate cravings are overall higher in the loss of control mindset as compared to a control mindset and mostly at T3, which is directly after the manipulation. The main effect of time seems mostly driven by a drop in craving between T4 and T5, which can be linked to the chocolate consumption. ${ }^{6}$ Figure 2 gives the means and standard errors per time point per condition.

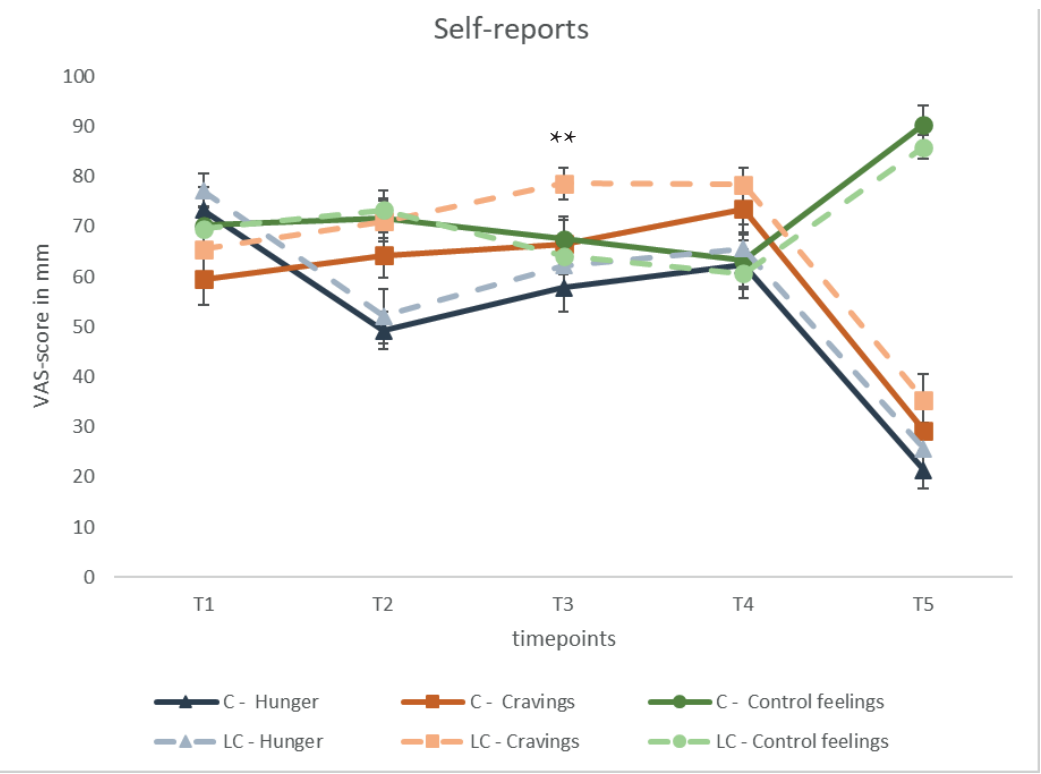

Figure 2. State control, craving and hunger for the 5 timepoints. Solid lines represent the Control condition, whereas dotted lines reflect the Loss of control condition. Error bars represent standard error of the mean. ${ }^{* \star}=p<0.01$ on paired t-test for craving. Abbreviation: VAS $=$ Visual Analogue Scale, C = Control condition, LC = Loss of Control condition.

\footnotetext{
${ }^{6}$ Four measurements were missing, one per participant, for the repeated measures ANOVA and one for the t-test.
} 


\section{Bogus chocolate taste test}

In line with our hypothesis, participants consumed significantly more chocolate in the loss of control mindset condition $(M=182.81, S D=68.30)$ than in the control mindset condition $(M=134.68, S D=61.57), t(25)=3.887, p=0.001, d=0.76$.

\section{Hormone levels}

Of the ghrelin dataset 12 samples ( 6 in lost of control and 6 in control condition) were not included in the analysis, as these could not be accurately determined (inter-assay variability $>15 \%$ ). These missing data were imputed with the series mean of the specific timepoint within the condition. Statistical tests were performed with and without the imputed values and did not result in different interpretations of findings. Twenty participants were included for the GLP-1 analysis, because the last 6 participants' GLP-1 values were unreliably determined due to a batch error. Ghrelin and GLP-1 both show a main effect of time (ghrelin: $F(4,22)=9.22, p<0.001, \eta_{p}^{2}=$ 0.63 and GLP-1: $\left.F(4,16)=17.93, p<0.001, \eta_{p}{ }^{2}=0.82\right)$. There is, however, no significant main effect of mindset (ghrelin: $F(1,25)=0.757, p=0.393, \eta_{p}^{2}=0.029$ and GLP-1: $\left.F(1,19)=1.93, p=0.18, \eta_{p}{ }^{2}=0.09\right)$ and also the mindset $x$ time interactions are not significant (ghrelin: $F(4,22)=0.48, p=0.75, \eta_{p}^{2}=0.08$ and GLP-1: $F(4,16)=0.45, p=$ $\left.0.77, \eta_{p}^{2}=0.10\right)$. Hormone responding was generally in line with chocolate intake. The means per timepoint per condition are shown in Figure 3.

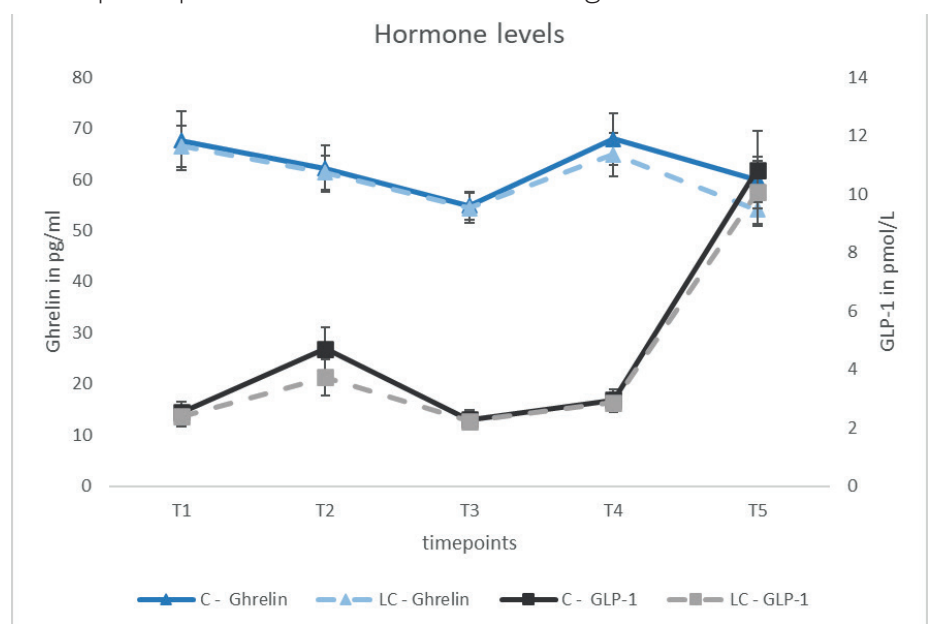

Figure 3. Hormonelevels of active grelin and GLP-1 on the 5 timepoints. Solid lines represent the Control condition, whereas dotted lines reflect the Loss of control condition. Error bars represent standard error of the mean. Abbreviations: VAS = Visual Analogue Scale, C = Control condition, LC = Loss of Control condition. 


\section{Whole-brain univariate analysis}

We tested the main effects of mindset (control vs no control) and stimulus type (chocolate and neutral) and the mindset $x$ stimulus type interaction wholebrain. The main effect of mindset and the mindset $x$ stimulus type interaction were not significant (all voxels $p>0.05$, FDR-corrected). A main effect of stimulustype (chocolate $>$ neutral) was found, including brain regions of the mesocorticolimbic system, which is known to be involved in craving and food-cue reactivity (Giuliani et al., 2018): insula, OFC, amygdala, putamen, lateral occipital cortex (LOC) $(p<0.05$, FDR corrected). See Table 2 and Figure 4 for a more detailed report of significant clusters. Therefore, in several regions of the mesocorticolimbic system, the neural response for chocolate stimuli was larger than for neutral stimuli, and this effect was not moderated by the mindset induction.

Table 1. Significant clusters from mass-univariate analyses including stimuli and mindset. Significant clusters of the main effect of stimulus type ( $p<0.05$, FDR-corrected) A cluster-extent threshold of 15 voxels was imposed, to only include the more robust clusters for interpretations.

\begin{tabular}{|c|c|c|c|c|c|c|c|}
\hline \multirow{2}{*}{\multicolumn{2}{|c|}{ Cluster }} & \multirow[t]{2}{*}{ hemisphere } & \multicolumn{3}{|c|}{$\begin{array}{l}\text { Peak MNI- } \\
\text { coordinates }\end{array}$} & \multirow[t]{2}{*}{$\begin{array}{c}\text { Peak } \\
\text { t-value }\end{array}$} & \multirow[t]{2}{*}{$\begin{array}{l}\text { Clustersize } \\
=\mathrm{k}\end{array}$} \\
\hline & & & $x$ & $\mathrm{y}$ & Z & & \\
\hline \multicolumn{8}{|c|}{ Chocolate > Neutral } \\
\hline 1 & LOC & $\mathrm{R}$ & 32 & -93 & 2 & 9.69 & 3393 \\
\hline 2 & LOC & L & -19 & -99 & -8 & 11.01 & 2832 \\
\hline 3 & Insula & L & -39 & -6 & 5 & 8.17 & 399 \\
\hline 4 & Insula & L & -40 & 4 & -6 & 7.23 & 366 \\
\hline 5 & Insula & $\mathrm{R}$ & 38 & -2 & 2 & 7.55 & 329 \\
\hline 6 & OFC & $\mathrm{R}$ & 22 & 29 & -10 & 7.05 & 233 \\
\hline 7 & Putamen & $\mathrm{R}$ & 35 & 9 & -8 & 7.07 & 150 \\
\hline 8 & Posterior Cingulate Cortex & L & -2 & -49 & 21 & 6.62 & 83 \\
\hline 9 & Fusiform area & $\mathrm{R}$ & 47 & -57 & -11 & 5.55 & 66 \\
\hline 10 & Subgenual Cingulate Area & L & -2 & 35 & 5 & 7.39 & 62 \\
\hline 11 & OFC & L & -31 & 36 & -10 & 6.16 & 47 \\
\hline 12 & Amygdala & L & -16 & -3 & -16 & 6.60 & 46 \\
\hline 13 & OFC & L & -25 & 31 & -10 & 5.59 & 44 \\
\hline 14 & Posterior Parietal Cortex & $\mathrm{R}$ & 47 & -31 & 37 & 5.21 & 43 \\
\hline 15 & OFC & L & -27 & 32 & -5 & 5.66 & 40 \\
\hline 16 & Cerebellum & L & -17 & -62 & -23 & 6.54 & 37 \\
\hline 17 & LOC & L & -13 & -93 & -21 & 7.95 & 36 \\
\hline 18 & Midbrain & $\mathrm{R}$ & 6 & 4 & 2 & 6.98 & 35 \\
\hline
\end{tabular}


Table 1.(Continued)

\begin{tabular}{|c|c|c|c|c|c|c|c|}
\hline \multicolumn{2}{|c|}{ Cluster } & \multirow[t]{2}{*}{ hemisphere } & \multicolumn{3}{|c|}{$\begin{array}{l}\text { Peak MNI- } \\
\text { coordinates }\end{array}$} & \multirow[t]{2}{*}{$\begin{array}{l}\text { Peak } \\
\text { t-value }\end{array}$} & \multirow[t]{2}{*}{$\begin{array}{l}\text { Clustersize } \\
=\mathrm{k}\end{array}$} \\
\hline & & & $x$ & y & Z & & \\
\hline 19 & Dorsal ACC & $\mathrm{R}$ & 1 & 32 & 18 & 5.40 & 34 \\
\hline 20 & LOC & L & -32 & -96 & -1 & 5.29 & 21 \\
\hline 21 & Caudate & L & -21 & 30 & -5 & 5.52 & 18 \\
\hline 22 & Dorsal ACC & $\mathrm{R}$ & 0 & 33 & 14 & 5.34 & 18 \\
\hline 23 & LOC & L & -29 & -90 & 1 & 4.83 & 18 \\
\hline 24 & Amygdala & $\mathrm{R}$ & 14 & -2 & -15 & 5.71 & 17 \\
\hline 25 & LOC & $\mathrm{R}$ & 35 & -84 & 11 & 5.28 & 17 \\
\hline 26 & Hippocampus & L & -14 & -10 & -15 & 5.31 & 16 \\
\hline 27 & Posterior Parietal Cortex & $\mathrm{R}$ & 59 & -16 & 27 & 5.04 & 16 \\
\hline 28 & LOC & L & -32 & -81 & -21 & 5.21 & 15 \\
\hline \multicolumn{8}{|c|}{ Neutral > Chocolate } \\
\hline 1 & LOC & L & -9 & -101 & 7 & 7.85 & 632 \\
\hline 2 & LOC & $\mathrm{R}$ & 10 & -93 & 6 & 7.23 & 335 \\
\hline 3 & LOC & $\mathrm{R}$ & 4 & -77 & -3 & 5.80 & 105 \\
\hline 4 & LOC & $\mathrm{R}$ & 8 & -78 & -11 & 6.21 & 58 \\
\hline 5 & LOC & $\mathrm{R}$ & 8 & -62 & 45 & 7.05 & 47 \\
\hline 6 & $\begin{array}{l}\text { Superior Parietal Lobule/ } \\
\text { Precuneus }\end{array}$ & $\mathrm{R}$ & -17 & -92 & 18 & 7.21 & 44 \\
\hline 7 & LOC & $\mathrm{R}$ & -9 & -78 & -14 & 5.56 & 36 \\
\hline 8 & Supplementary Motor Area & $\mathrm{R}$ & 24 & 5 & 49 & 6.17 & 35 \\
\hline 9 & LOC & $\mathrm{R}$ & 8 & -73 & 3 & 5.60 & 28 \\
\hline 10 & LOC & L & -4 & -75 & -8 & 6.28 & 24 \\
\hline 11 & LOC & $\mathrm{R}$ & 8 & -75 & 9 & 6.17 & 17 \\
\hline 12 & LOC & L & -19 & -75 & -15 & 4.77 & 15 \\
\hline
\end{tabular}

Abbreviations: $\mathrm{L}=$ left, $\mathrm{R}=$ right, $\mathrm{B}=$ Bilateral, $\mathrm{MNI}=$ Montreal Neurological Institute. $\mathrm{OFC}=$ orbital frontal cortex, ACC = anterior cingulate cortex, LOC = lateral occipital complex. 


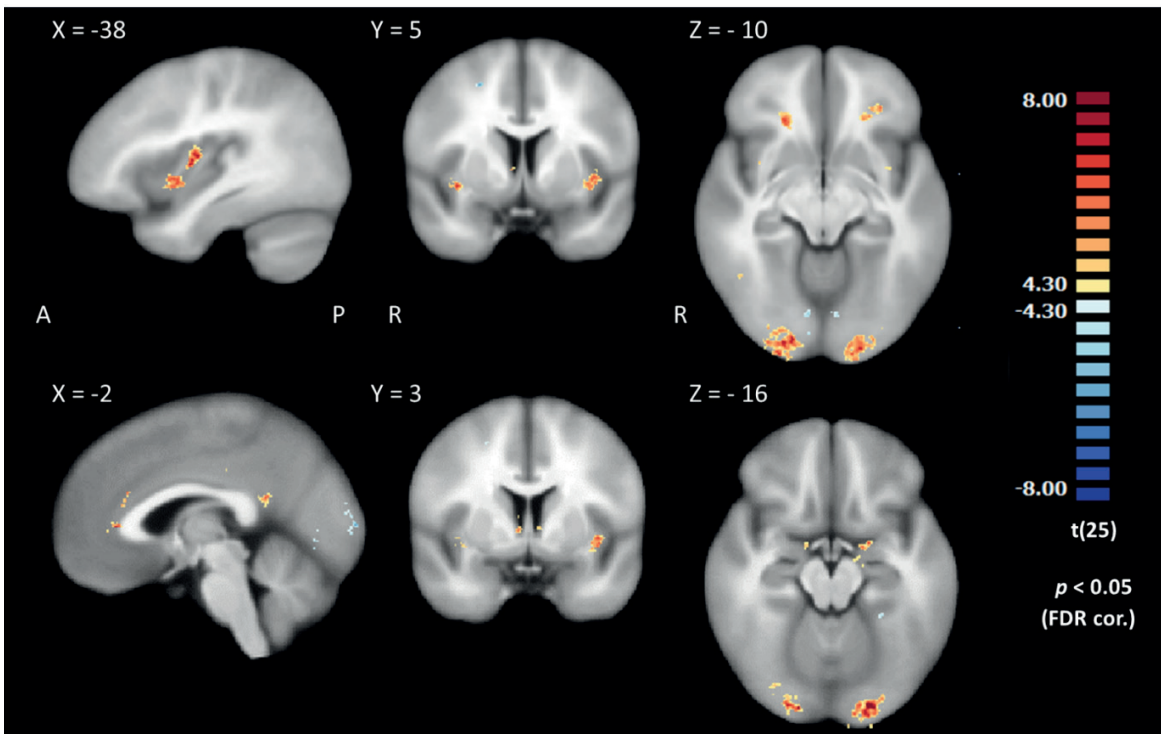

Figure 4. Whole brain results from mass-univariate analyses. $t$-maps of signifi cant main eff ects of stimulus type collapsed over mindset; hot maps represent chocolate stimuli $>$ neutra stimuli and cold maps represent neutral stimuli $>$ chocolate stimul, all maps are $p<0.05$ FDR corrected. Clusters are presented on a MNI template with the $x-y-z$ MNI-coordinates.

\section{Correlation neural activity with chocolate cravings}

To test whether trait chocolate craving was associated with neural activity, we entered the trait chocolate craving (ACQ) score as a covariate in our original GLM for univariate analysis with mindset (control vs no control) and stimulus type (chocolate and neutral). ACQ scores did not correlate with the whole-brain main effect of stimulus type (all voxels $p>0.05$, FDR-corrected). Therefore, trait chocolate craving was not significantly related to neural responses to visual chocolate stimuli.

To test if a significant correlation between trait chocolate craving and neural activity would be observed right after the mindset induction, we entered the state craving scores at timepoint 3 (directly after both mindset inductions) as 2 covariates for each mindset in our original GLM for univariate analysis. Again, no signifi cant correlation between trait chocolate craving and neural responses to stimulus was observed (all voxels $p>0.05$, FDR-corrected). 


\section{Whole-brain multivariate analysis}

Using a whole-brain searchlight approach (Kriegeskorte et al., 2006), we tested if stimulus type (chocolate versus neutral) could be decoded above chance from patterns of neural activity by calculating classification accuracies, using a $6 \mathrm{~mm}$ radius sphere, per voxel. We expected that classification of stimulus type (chocolate versus neutral) would be more accurate for the loss of control mindset in the mesocorticolimbic system, whereas we expected classification to be more accurate for the control mindset in control-related brain areas.

Within both mindsets, stimulus type could be decoded significantly above chance (voxel-wise FDR corrected). The clusters largely overlapped with the results of the mass-univariate analysis of the main effect of stimulus type (Figure 5), including areas in the bilateral (dorsal and ventro-lateral) prefrontal cortices, insulae, amygdalae, parietal cortices and lateral occipital cortex (LOC).

We subsequently tested whether this decoding accuracy differed between mindset conditions. However, no clusters had a higher decoding accuracy in the loss of control as compared to the control mindset condition or in the opposite direction after voxel-wise FDR correction. So, stimulus type could be significantly decoded above chance per condition, but decoding accuracy did not differ significantly between conditions. 


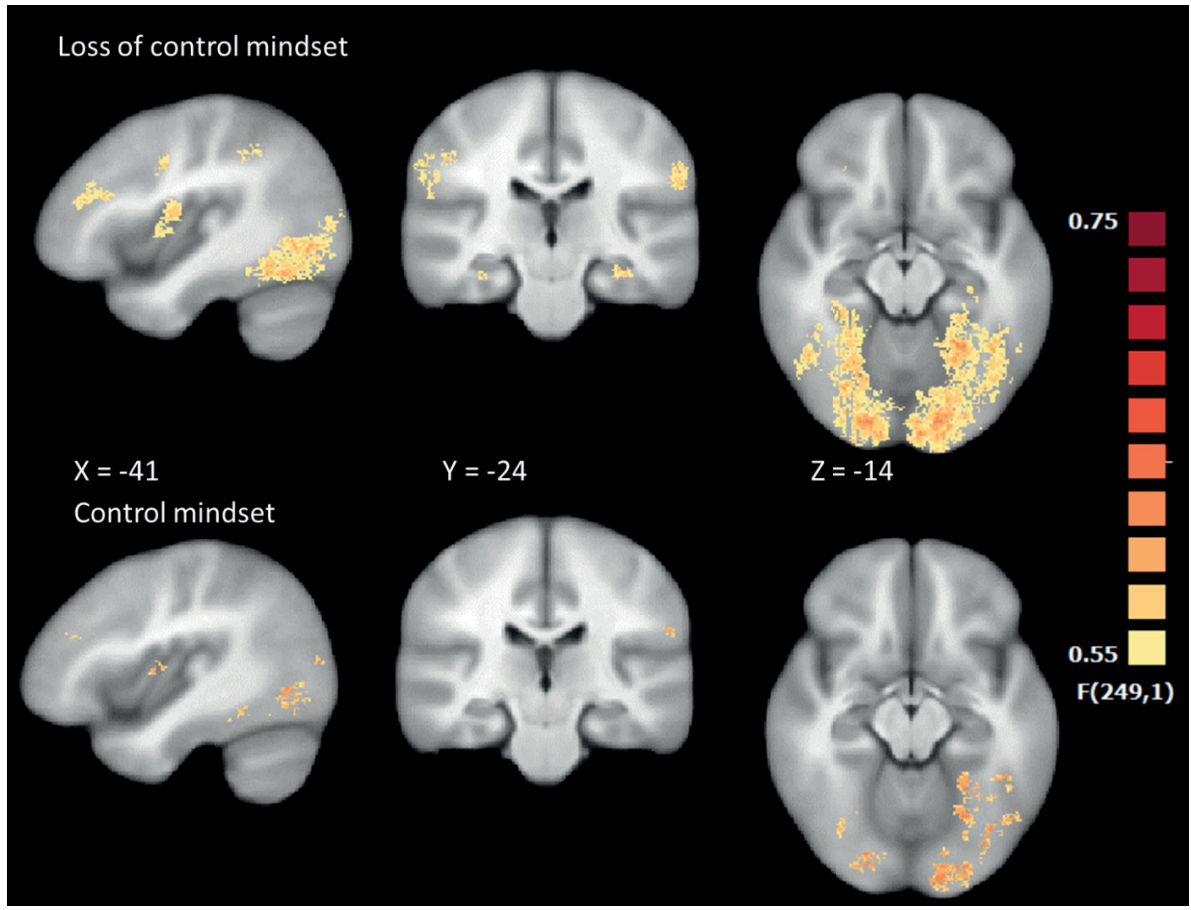

Figure 5. Neural representation of stimulus type. Clusters with significant classification accuracy of food palatability as derived from whole brain searchlight analysis for loss of control and control mindset condition. Clusters are presented on a MNI temlate with the $\mathrm{x}-\mathrm{y}-\mathrm{z} \mathrm{MNI}$ coordinates. These significant accuracy clusters were tested against $50 \%$ chance with Wilcoxon signed rank tests ( $p<0.05$, FDR corrected, clustersize threshold: 50 for robust clusters and display purposes).

\section{Discussion}

The aim of this study was to examine the influence of a loss of control versus a control mindset on hormonal and neural responses, self-reported cravings, hunger, control and chocolate intake. The current study shows that (1) neural activity in several regions of the mesocorticolimbic system is stronger for chocolate stimuli than for neutral stimuli, and this effect is not moderated by mindset, (2) ghrelin and GLP1 levels respond to food intake, but they are not affected by mindset, and (3) selfreported craving and actual chocolate intake are affected by mindset, in that cravings and intake are higher with a loss of control mindset than with a control mindset. So, subjective experience and behaviour are affected, whereas physiological and neural responses are not influenced by the induced mindset. 
The predicted mindset effect observed for momentary craving and chocolate intake, is in line with previous studies investigating cognitive modulations of eating behaviour, showing that beliefs generated by health claims or caloric content labels of high caloric food products influence intake (e.g., McCann et al., 2013; Roefs \& Jansen, 2004; Shide \& Rolls, 1995). This is also in line with previous research that showed that a health mindset attenuates attention towards high caloric foods (Werthmann et al., 2016). Recent studies also showed that food cravings and food valuation are affected by cognitive strategies, like regulation or reappraisal of food cravings (Boswell et al., 2018; Reader et al., 2018). In contrast with these previous studies, our manipulation was not directed at the food product itself (e.g., with caloric content labels), instead we aimed to induce a more general state of mind of "letting yourself go" in the loss of control mindset versus "being in control" in the control mindset. Our data fit with cognitive models of overeating and binge eating, demonstrating that beliefs are strong determinants of behavior (see e.g., Beck, 2009).

In this study, we did not find any significant top-down neural control-related responses after inducing a control mindset, nor any indications for stronger neural craving-related responses by inducing a loss of control mindset. A possible explanation for not finding the hypothesized effect, is that all participants scored high on trait chocolate craving. Seeing chocolate stimuli might have triggered neural activity in the mesocorticolimbic system, independent of the induced mindset (Giuliani et al., 2018). The automatic response might be stronger for these high trait chocolate cravers than a quite subtly induced mindset. There is indeed some evidence (Miedl et al., 2018) that high trait chocolate cravers show more implicit pleasure related neural responses in the mesocorticolimbic system towards chocolate pictures as compared to low chocolate cravers, without strict instructions during viewing.

However, when chocolate cravers were explicitly instructed to suppress their chocolate craving thoughts while viewing chocolate pictures, both the craving and activity in the mesocorticolimbic system were reduced (Miedl et al., 2018). This finding converges nicely with recent work from our laboratory, showing that an explicitly induced attentional focus (i.e. 1-back task with focus on taste versus colors) while viewing food images, strongly influenced participants' neural responses to highcaloric food pictures (Franssen et al., 2020). That is, the neural response in the mesocorticolimbic system was significantly higher with a hedonic than with a neutral 
focus, independent of the palatability of the presented food items. So, a second explanation for not finding the predicted effects in the current study, could be that the mindset induction was not as explicit and it was not part of task requirements (as with our 1-back task in Franssen et al., 2020).

Third, some studies indicate a difference between anticipatory and consummatory food reward processing (Small, 2009; Small et al., 2008) Where anticipatory food reward entails the anticipated rewarding value of a food cue, consummatory food reward is the experienced rewarding value after actual intake. It could be that this type of induced mindsets would interfere with neural responses during scanning when participants also correspondingly receive actual chocolate (or chocolate milk). So, our control / loss of control mindsets might have affected consummatory reward instead of anticipatory rewards, as we did find differences in actual eating behavior (i.e., chocolate consumption during the bogus taste test).

As expected, independent of mindset, we did find increased neural activity to chocolate as compared to neutral stimuli in brain regions that have previously been reported to be involved in food-craving or food-cue reactivity (e.g., insula, OFC, amygdala, putamen, lateral occipital cortex (LOC)) (Giuliani et al., 2018; Pohl et al., 2017; Suzuki et al., 2017; van der Laan et al., 2011). This is different compared to our previous results, where we did not find any neural differences between food stimuli (Franssen et al., 2020). However, an important big difference to the previous study is the visual or motivational saliency of the stimuli presented. The food stimuli used in the previous study were equally salient to the participants (individually tailored highly palatable and highly unpalatable foods) as confirmed by not finding any differences in univariate neural responses earlier (Chikazoe et al., 2014). In the current study, chocolate pictures are likely being processed as more salient than the presented non-food office supplies stimuli, and this saliency seem to be even more pronounced for this specific group of high chocolate cravers. Our univariate neural findings therefore suggest that the saliency of chocolate stimuli overrules the possible effect of the induced - and less explicitly active and stimuli-engaged - loss of control or control mindset.

Whereas mass univariate analyses informs on involvement of activated brain areas, multivariate analyses inform on the representational content of these brain areas (Norman et al., 2006). Though the decoding accuracy of chocolate versus neutral 
stimuli was significantly above chance, decoding accuracies did not differ significantly between mindsets. Decoding of chocolate versus neutral stimuli is in line with prior research, which showed that the subjective value of food stimuli could be decoded from multi-voxel patterns of neural activity (Chikazoe et al., 2014; Suzuki et al., 2017). The multivariate analyses suggest that the subjective value of chocolate stimuli was not altered by the induced mindsets while processing the chocolate stimuli. In our previous study (Franssen et al., 2020), we did observe that decoding accuracy of highly palatable versus highly unpalatable stimuli was significantly better for the hedonic focus than for the neutral focus. Again, the explanation could be that in the previous study the induction of mindset was very explicit and task-based (i.e., 1-back task with a focus on taste versus color).

Hormonal responses did not confirm our hypothesis either; no significant differences were found in ghrelin and GLP-1 responses between the two induced mindsets. Hormones responded as expected over time and in response to eating moments, where ghrelin levels decreased, and GLP-1 levels increased after eating moments. The findings on anticipated hormone levels are however not in line with previous studies, finding expectations or beliefs to influence hormonal responding to food (Cassady et al., 2012; Crum et al., 2011). A difference with the current study is that in the studies of Cassady et al (2012) and Crum et al (2011) expectations were directly linked to a specific food or drink, by attached labels, brands or productspecific caloric density expectations. We induced a more general mindset, and we did not induce any expectations about the satiating effects or caloric load of the eaten chocolate. Whereas higher chocolate craving was reported after the loss of control mindset, this was not accompanied by self-reported hunger or hormone levels. This might suggest that priming participants' mindset or beliefs about specifically calorie content or satiating values of foods or drinks is needed to change hunger feelings and the accompanied hormone release.

Taken together, the current study gives some interesting leads for further investigation. This study illustrates that control and loss of control mindsets influence chocolate cravings and chocolate intake, whereas neural and hormonal responses are not influenced by the induction of these specific mindsets. One of the strengths of our study is the multidisciplinary set up, including several psychological and physiological measures. An ultra-high field (7T) MRI scanner was used to acquire fMRI data, which made it possible to examine neural responses in great detail. Moreover, fMRI data 
were analyzed using both mass-univariate and multivariate analyses. Participants served as their own controls, which made it a strong within-subjects design, as the experimental procedure was kept exactly the same in both sessions, except for the mindset induction. The group of participants was homogeneous, consisting of female chocolate cravers, thereby reducing variability. The disadvantage is that the results of the current study are less generalizable to other food cravings and to other populations (e.g., males). Though further research is needed to confirm and expand these findings, the current study shows that cravings and intake can be manipulated by a quite subtle mindset induction.

\section{Acknowledgements}

This study was financed by the Netherlands Organization for Scientific Research (NWO), FCB-grant (057-13-010) and the Maastricht University Interfaculty Program 'Eatwell', both awarded to Anita Jansen. The authors would like to acknowledge Kyra Wijnen and Vincent van de Vlasakker for their assistance in data collection.

\section{Author Contributions}

S.F., Anne R. T.A. and A.J. designed the study. S.F. collected the data. S.F. and J.H. analyzed the data. The scanning protocol was set up by Alard R. S.F. and Anne R. wrote the manuscript, T.A., A.J., Alard R., and J.H. gave feedback on the manuscripts, and all authors approved the final version.

\section{Conflicts of interests}

The authors declare no conflicts of interest 



\section{CHAPTER 4}

\section{YOUR MIND ON SWEETTASTE: EFFECTS OF ANTICIPATED CALORIC LOAD ON METABOLIC RESPONSETO CARBOHYDRATE CONSUMPTION}

Submitted as: Franssen, S., Roefs, A., Raithel, C., Jansen, A., and Small, D., Your mind on sweet taste: Effects of anticipated caloric load on metabolic response to carbohydrate consumption. Physiology and Behavior 


\section{Abstract}

Non-nutritive sweeteners (NNSs) are often used as replacements for sugars because they provide the same liked sweet taste without containing any calories. However, NNS have been associated with metabolic dysfunction, which might be caused by a mismatch between the expected caloric content as indicated by the sweetness and the actual caloric content. In this study, it is investigated if manipulating the expected caloric content directly by means of food labels also affects glucose metabolism. In a within-subject design ( $n=17)$, resting energy expenditure (REE) and subjective experiences (i.e., liking, hunger, fullness, and thirst) were measured before and after consuming an isocaloric beverage that was labelled as "low", "mid" or "high" caloric. Unexpectedly, REE and subjective experiences did not significantly differ between the three label conditions. The metabolic response was only influenced by actual caloric load, with a larger REE post than pre beverage consumption. These findings suggest that metabolic responses are not sensitive to caloric content anticipations as induced by labels.

\section{Keywords}

Metabolic response, caloric content anticipation, nutrition, taste 


\section{Introduction}

Overconsumption of high caloric foods and drinks are major contributors to weight gain, obesity and type 2 diabetes (Hall et al., 2012; Mitchell et al., 2011). Sugar sweetened beverages (SSBS) have received much attention in research and are considered an important contributor to the obesity epidemic ( $H u$ \& Malik, 2010). Non-nutritive sweeteners (NNSs), for instance used in diet sodas, were put forward as healthy substitutes for sugars as they provide the same liked sweet taste without containing any calories. However, some epidemiological studies have suggested that long-term consumption of NNS beverages is associated with similar adverse effects as SSBS (e.g., weight gain and type 2 diabetes). Therefore, using NSS as an healthy substitute is heavily debated (Dalenberg et al., 2020; Imamura et al., 2015; Pepino, 2015; Romo-Romo et al., 2016; Swithers et al., 2010).

Although there are many potential mechanisms by which NNS use could promote metabolic dysfunction, several groups have highlighted a role for sweet taste perception. The perception of sweetness produces, "cephalic phase responses" (Abdallah et al., 1997) that help prepare the organism for energy metabolism. The uncoupling hypothesis (Davidson \& Swithers, 2004) argues that the uncoupling of sweet taste from calories reduces the effectiveness of sweetness as a predictive signal, and thus diminishing cephalic phase responses, which overtime produces metabolic dysfunction. Similarly, Veldhuizen and colleagues (2017) showed that sweetness perception alters the metabolic fate of a standard carbohydrate load. More specifically, the metabolic response to an 112.5Kcal beverage differed depending on the amount of sucralose present (and therefore the level of sweetness) in the drink, with sweetness either corresponding to $112.5 \mathrm{kcal}$ (matched) or to $75 \mathrm{kcal}$ (mismatch). The metabolic response was lower for the mismatched than for the matched drink. The authors therefore proposed that sweet taste regulates carbohydrate metabolism (Veldhuizen et al., 2017) and suggested that mismatches between sweet taste perception and caloric load has the potential to produce metabolic dysfunction as physiological circuits adapt to alterations in sweet taste - calorie associations.

Consistent with a role for sweet taste perception, NNS consumption alters glucose metabolism both acutely (Pepino, 2015; Veldhuizen et al., 2017) and after habitual consumption (Dalenberg et al., 2020). Collectively, this work suggests that sweet taste plays a role in glucose metabolism. However, whether this effect 
is inherent to sweet perception or whether other cognitive signals, such as beliefs and expectations about caloric load might also influence glucose metabolism is still unclear. Therefore, the central aim of this study is to examine if expectations of caloric content influence metabolic response following consumption of carbohydrate containing beverages.

Consistent with this idea, prior work has demonstrated that expectations of caloric content can influence ingestive behaviour and physiological responses. For example, the release of the hunger-related hormone ghrelin (Kojima et al., 1999) decreased more strongly when participants thought they had consumed an indulgent high caloric milkshake than when they thought they had consumed a sensible low caloric milkshake, while in fact the milkshake was the same in both conditions (Crum et al., 2011). Another study showed similar metabolic effects on glucagon like peptide-1 (GLP-1, i.e. hormone that reflects satiety) release when manipulating satiety expectations (solid versus liquid) of consumed beverages. When the beverage was expected to be a more satiating preload (solid condition), an increased GLP-1 release was found after consumption, as compared to when the beverage was expected to be a normal liquid preload (liquid condition) (Cassady et al., 2012). In addition, labels on food products (i.e. healthy or hedonic labels) can influence liking and perceived healthiness (Provencher \& Jacob, 2016) and hypothalamic response to flavored beverages (Veldhuizen et al., 2013). Accordingly, caloric intake is generally reduced when consuming food with a higher perceived caloric content as compared to a lower perceived caloric content (Faulkner et al., 2014; Gravel et al., 2012; Hogenkamp et al., 2013). These findings indicate that beliefs and expectations can play a significant role in physiological hunger and satiety and can have consequences for consumption and metabolism.

However, whether, like the sense of sweet taste, anticipated caloric properties also influence metabolic response following consumption of sweetened beverages is not clear. Therefore, the central aim of this study was to use indirect calorimetry to measure the metabolic rate, termed the thermic effect of food (TEF), following ingestion of carbohydrate-containing beverages and test if the TEF differs depending on caloric labels. More specifically, indirect calorimetry was used to measure resting energy expenditure (REE) before and after the consumption of three beverages containing the same caloric load (112.5 kcal from maltodextrin) and sweetness (0.0078\% sucralose) but labelled as "low", "mid" and "high" calorie content. If expected caloric content influences nutrient metabolism, then the TEF should differ depending upon 
label such that larger responses are associated with expectations of more calories. We also tested if differences in internal state experiences (hunger, fullness and thirst) were associated with TEF. Predicted was that labels of higher calorie content would result in a stronger decrease in hunger and increase in fullness following beverage consumption.

\section{Methods and materials}

\section{Participants}

Thirty-two healthy-weight non-smoking participants (BMl 18.5-25 kg/m²) were recruited in New Haven, Connecticut, USA. All participants gave informed consent and the study was approved by the Yale University School of Medicine Human Investigation Committee. All participants underwent an initial screening session, which included several taste tasks to ensure perception of the beverages fell within a target range and to insure participants were non-regular users of NNSS (see 2.2. Procedure). Seventeen participants were included in the study. The sample size was based on previous studies in the same research domain using similar analysis approaches (Veldhuizen et al., 2017). Assuming a large effect size, 80\% power, and alpha of 0.05 (calculated with $G^{*}$ power 3.0. see: Faul, Erdfelder, Buchner, \& Lang, 2009), the required sample size is 12 .

\section{Procedure}

\section{Pre-test}

Prior to study commencement, all participants attended a two-hour pre-test and screening session. As part of this procedure, participants were first trained to use the general Labeled Magnitude Scale (gLMS; Green et al., 1996), Labeled Hedonic Scale (LHS; Lim, Wood, \& Green, 2009), and visual analogue scales (VAS), needed to complete their perceptual ratings during the measurement sessions. Following training, ten flavoured and coloured non-caloric sweetened beverages (see 2.3.2. Stimuli) were presented three times for a robust measurement (counterbalanced in pseudo-random orders) and rated for overall intensity, sweetness, familiarity, wanting, and liking. Participants who did not rate at least three out of the ten flavour-colour beverages as moderately and roughly equally liked, were excluded from participation. Participants meeting this requirement then performed a triangle test to determine 
if they could detect the presence of maltodextrin in the beverage. Maltodextrin is a glucose polymer that produces minimal oral sensation and is generally not detectable in flavoured solutions (Veldhuizen et al., 2013 and 2017). To verify that participants could not detect the maltodextrin by oral sensation, they were asked to sample cups containing the same flavour and indicate which of three was different from the others. On any given trial, 1 or 2 of the cups contained maltodextrin. Eight trials were conducted and the binomial distribution of chance was used to set our criteria for maltodextrin detection as 6 or greater correct responses (Veldhuizen et al., 2017). Finally, height and weight were measured, and participants filled out the Nutrition and Beverage Use Questionnaire (NBUQ) to determine how frequently they consumed foods containing artificial sweeteners. The NBUQ was administered to ensure all participants reported consuming artificial sweeteners no more than three times a month. Those who endorsed consuming more were excluded.

\section{Experimental sessions}

Participants underwent three experimental indirect calorimetry (IC) measurements (one-hour each) in a within-subject design on three separate days. In each session, one of the three flavoured beverages was presented (see 2.3.2. Stimuli). Sessions were conducted in the late morning (between $9.30 \mathrm{am}$ - 12 pm), and participants were asked to arrive fasted for at least four hours. The three sessions were completed within two weeks. Participants were informed that the purpose of the study was to test whether the consumption of a sweetened low-, medium- or high-caloric beverage differentially influences the metabolic response. Before (15 minutes) and after (30 minutes) each IC session, ratings of internal state (see paragraph 2.3.3.) were collected.

\section{Materials}

\section{Indirect Calorimetry (IC)}

To measure TEF, resting energy expenditure (REE) was measured using IC with a TrueOne 2400 Metabolic Measurement System. The system measures the ratio of carbon dioxide production over oxygen consumption from the expired air for every minute of the testing session to calculate the REE (Reed \& Hill, 1996; Weir, 1949). A plastic transparent metabolic canopy was placed over both the participant's head to neck to limit the space from which carbon dioxide production and oxygen 
consumption was measured. While measuring IC, participants were in a lying position and instructed to relax without falling asleep. REE was measured before beverage consumption for 15 min and after consumption for 30 min, respectively: pre-beverage and post-beverage REE. To standardize the REE timing, participants were asked to consume the $355 \mathrm{ml}$-beverage within 2 minutes.

\section{Stimuli}

Stimuli used for the pre-test were ten differently flavoured non-caloric beverages, containing $0.1 \%(\mathrm{~W} / \mathrm{V})$ citric acid and $0.0078 \%(\mathrm{~W} / \mathrm{V})$ sucralose (Sigma-Aldrich MO, USA) dissolved in demineralized water. Although all beverages had the same sweetness intensity, each had a unique randomly assigned flavour and colour, counter-balanced across subjects so that there were no consistent flavour-colour pairings. The flavours used in this experiment were $0.002 \%$ acerola, $0.5 \%$ bilberry, $0.1 \%$ horchata, $0.1 \%$ lulo, $0.2 \%$ yuzu, $0.1 \%$ papaya, $0.1 \%$ chamomile, $0.1 \%$ aloe vera, $0.1 \%$ mamey, and $0.2 \%$ maqui berry (Bell Labs Flavors and Fragrances, IL, USA, product numbers: 33.81940, 15.80182, 132.81478, 141.14606. 101.29478, 102.82506, 141.31243, 141.31480, 46.29969 and 13.32059). Food colouring (colours from McCormick, MD, USA) was used to create pink, yellow, green, blue, purple, red, orange, teal, yellow-green or indigo beverages. For the maltodextrin triangle test, we mixed a SoBe lifewater ("mango melon", "black and blueberry", containing < 5 calories from PepsiCo, NY, USA) with maltodextrin (Spectrum, CA, USA) at 34\% (w/v) to the equivalent of 150 calories in $355 \mathrm{ml}$.

The three flavoured beverages rated as similarly liked at pre-test were used to create the drink set for each participant. Liking ratings were required to fall within "neutral" (0) and "like moderately" (17.82) on the LHS and standard deviations of all three were required to overlap. All had unique colour-flavour pairings to limit habituation (Benson \& Raynor, 2014) and to increase the distinctiveness and therefore credibility of the three caloric labels - although the same number of calories (112.5 kcal from maltodextrin) was added to each - resulting in three $355 \mathrm{ml}$ isocaloric and equally sweet beverages. The non-nutritive sweetener sucralose was used to sweeten the beverage to match the sweetness of a $112.5 \mathrm{kcal}$ drink containing sucrose. This caloric load was selected because TEF following its consumption was shown in a previous study to vary as a function of changes in sweetness ( Veldhuizen et al., 2017). Beverage presentation (i.e., label) was counter-balanced across days and subjects. 


\section{Internal state ratings}

Before each REE measurement session, hunger, fullness and thirst were rated on a vertical $100 \mathrm{~mm}$ visual analogue scale (VAS), ranging from "not hungry/full/thirsty at all" at the bottom and "very hungry/full/thirsty" at the top.

\section{Beverage stimuli ratings}

After beverage consumption, the beverage was rated on intensity, sweetness, sourness, bitterness using the general Labeled Magnitude Scale (gLMS). The gLMS consists of 100 units with labels placed at "barely detectable", 1.4; "weak", 6; "moderate", 17; "strong", 34.7; "very strong", 52.5; "strongest imaginable sensation of any kind", 100 (Bartoshuk et al., 2004; Green et al., 1996). Liking was measured using Labeled Hedonic Scale (LHS), which is constructed from the geometric means of magnitude estimates of 10 semantic descriptors with extreme ranging from "most imaginable disliking" -100 to "most imaginable liking" 100 (Lim et al., 2009)).

\section{Nutrition and Beverage Use Questionnaire}

Participants completed a Nutrition and Beverage Use Questionnaire (NBUQ) developed at The John B. Pierce Laboratory. This questionnaire entails an evaluation of four questions on how often participants used various artificially sweetened products, with answer options ranging from "never, or less than once/month" to "6 or more times a day" with a minimum score of zero and a maximum score of $24 .^{7}$

\section{Analysis}

To obtain a reliable measure of REE response, we created two 5-min measurement windows; the pre-beverage REE was based on the last 5 minutes of the baseline just prior to removing the hood for beverage consumption, and the post-beverage REE was based on the last 5-minute period beginning approximately 25-min after beverage consumption, which corresponds to the time that plasma glucose levels should peak (see Veldhuizen et al., 2017). To examine the interaction of time and label manipulation, the variables REE, hunger, fullness, and thirst were analysed

7 The questionnaire also assesses frequency of intake of many other foods, which were not included here. 
in 2 (time: pre versus post beverage consumption) $\times 3$ (label: low-, vs mid- vs highcaloric) repeated measures ANOVAs. When the assumption of sphericity was violated, Greenhouse-Geisser corrected $p$-values were reported. Bonferroni correction was used to adjust for multiple testing, and significant effects were further explored by paired-samples t-tests. TEF was calculated by subtracting the pre-beverage REE from post-beverage REE response (post REE response - pre REE response) for each condition. In addition, post - pre beverage consumption scores for hunger, thirst and fullness were calculated. These difference scores were used as input for correlational analyses. To examine associations between subjective experiences and TEF, correlational analyses were conducted with the differences scores of hunger, fullness and thirst and the difference scores of REE. Finally, the post-beverage consumption ratings of liking and beverage characteristics were analyzed in a 1-way repeated measures ANOVA with three levels (label: low- vs mid- vs high-caloric).

\section{Results}

\section{Participants' characteristics}

In total, 17 participants (9 females) qualified for inclusion. All participants selfreported no (history of) significant taste, smell, metabolic or psychiatric disorders. The participants' characteristics can be found in Table 1.

Table 1 Descriptive statistics of participants' characteristics

\begin{tabular}{lcccc}
\hline & $\mathbf{n}$ & $\mathbf{M}$ & SD & $\begin{array}{c}\text { Range (min- max) } \\
\text { or Ratio (F/M) }\end{array}$ \\
\hline Age (in years) & 17 & 25.24 & 4.70 & $19-33$ \\
Gender (\% female) & 17 & 52.94 & & $9 / 8$ \\
Education level (in years) & 16 & 15.63 & 2.39 & $12-22$ \\
BMl (kg/m2) & 17 & 22.26 & 1.89 & $19.02-24.90$ \\
Maltodextrin correct detection & 17 & 3.53 & 1.46 & $1-5$ \\
NBUQ-score & 17 & 3.06 & 0.24 & $3-4$ \\
\hline
\end{tabular}

Note: Abbreviations: $n=$ number of participants, $M=$ Mean, $S D=$ standard deviation, BMl= Body Mass Index, NBUQ = Nutrition and Beverage Use Questionnaire, F= Female, $M=$ Male 


\section{Behavioural ratings}

\section{Beverage stimuli ratings}

The ratings of intensity $\left(F(2,32)=0.38, p=0.68, \eta_{p}^{2}=0.02\right)$, sweetness $(F(2,32)=$ $\left.0.39, p=0.68, \eta_{p}^{2}=0.02\right)$, sourness $\left(F(2,32)=0.03, p=0.97, \eta_{p}^{2}<0.01\right)$, bitterness $\left(F(1.22,19.59)=2.61, p=0.12, \eta_{p}^{2}=0.14\right)$ and liking $\left(F(2,32)=0.16, p=0.86, \eta_{p}^{2}=0.01\right)$ did not significantly differ between the three different labelled beverages. See Table 2 for averages and standard deviations per rating.

Table 2 Descriptive statistics of beverage stimuli ratings per rating category for the perceived lowmid-and high-caloric beverages.

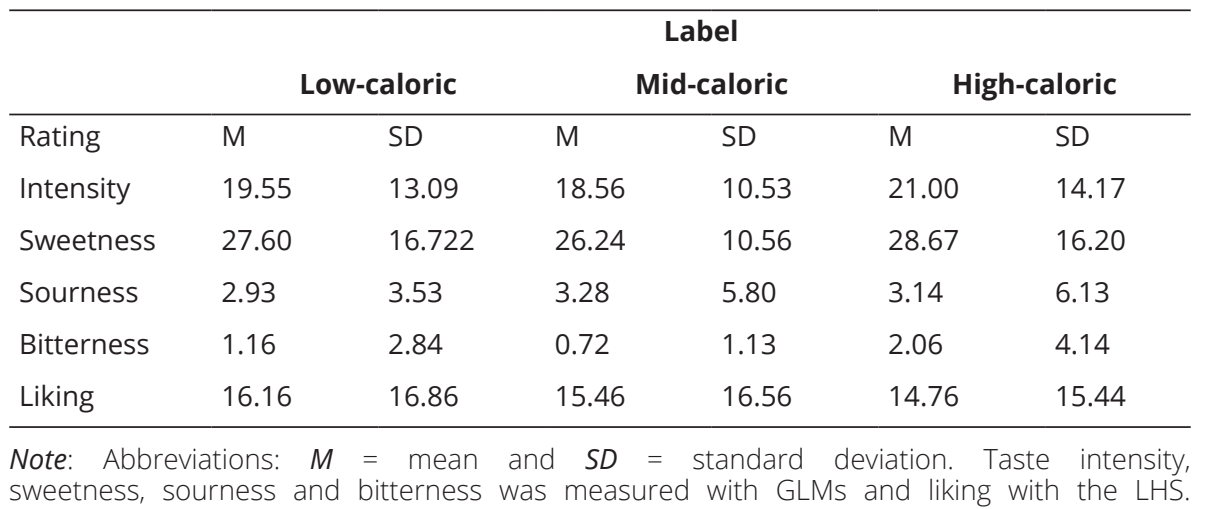

\section{Internal state ratings}

No time $x$ label interactions (all $F<0.977, p>0.39$ ), nor main effects of label (all $F<$ $2.51, p>0.10)$ for hunger, fullness and thirst were observed. There was a main effect of time on hunger $(F(1,16)=13.51, p<0.01, \eta p 2=0.46)$ and thirst $(F(1,16)=9.25, p<$ $0.01, \eta p 2=0.37)$ and a marginal significant effect of time on fullness $(F(1,16)=4.24, p$ $=0.06, \eta p 2=0.21)$. These finding indicate that, independent of caloric label, hunger and thirst were decreased and fullness was increased as response to beverage consumption (see Figure 1). 


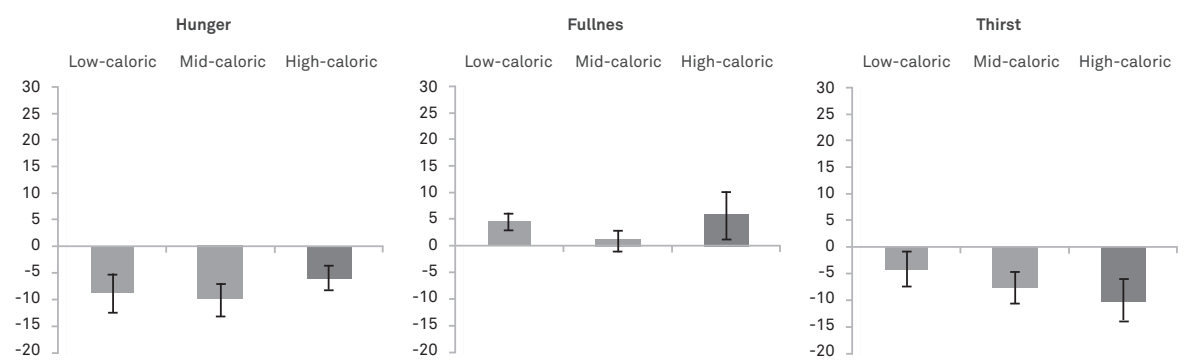

Figure 1. Internal state rating differences. Bar plots represents mean internal state rating differences with error bars (standard error of the mean) pre to post after consuming perceived low- mid- and high-caloric beverage per rating category measured with vertical $100 \mathrm{~mm}$ VAS scales.

\section{Indirect Calorimetry}

No time $\times$ label interaction $\left(F(2,32)=0.16, p=0.82, \eta_{p}^{2}=0.10\right)$ was observed, nor a main effect of label $\left(F(1.27,20.31)=0.11, p=0.80, \eta_{p}{ }^{2}<0.01\right)$ on REE. There was a main effect of time on $\operatorname{REE}\left(F(1,16)=21.82, p<0.01, \eta_{p}^{2}=0.58\right)$ see Figure 2$)$. These results indicate that REE increase was linked to beverage consumption, but this REE increase was independent of caloric label condition.
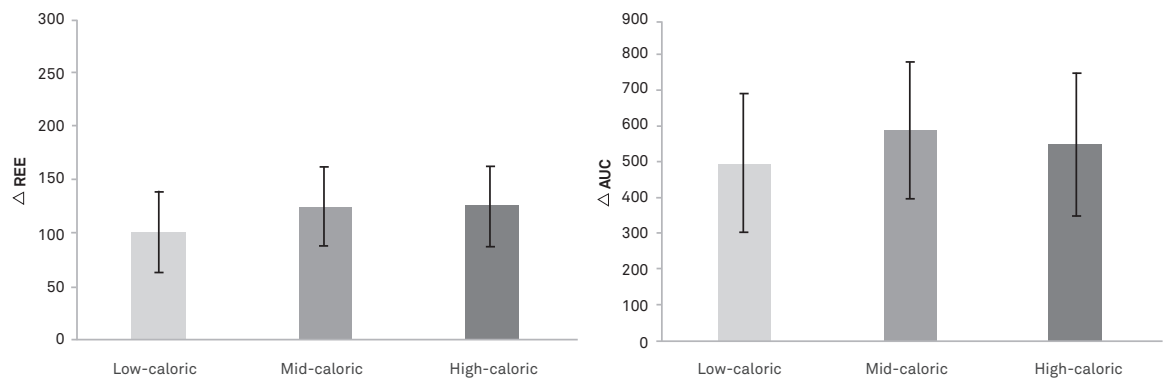

Figure 2. Thermic effect of food (TEF) of low- mid and high caloric labelled isocaloric sweetened beverages. Bar plots represent mean change in REE from pre to post beverage

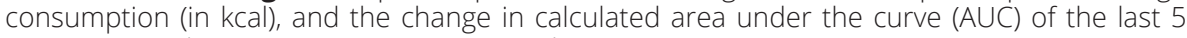
minutes (post-beverage consumption - pre-beverage consumption) as response to consuming a preceived low- mid-and high-caloric labelled beverage. All error bars reflect 1 standard error of the mean (+ and -).

To be certain that results were not biased by the specific analysis method, also area under the curve (AUC) analyses using the trapezoidal rule were performed. No time $x$ label interaction was observed on $\operatorname{A\cup C}\left(F\left(2,32=0.07, p=0.93, \eta_{p}{ }^{2}<0.01\right)\right.$, nor a 
main effect of label $\left(F(1.25,20.12)=0.05, p=0.87, \eta_{p}{ }^{2}<0.01\right)$. There was a main effect of time $\left.F(1,16)=16.34 p<0.01, \eta_{p}^{2}=0.50\right)$. So, the AUC-analyses are fully in line with the analyses on REE (in kcal).

\section{Correlations subjective internal state experiences and TEF}

To examine associations between difference scores (post - pre consumption) of subjective internal state experiences (hunger, fullness and thirst) and the TEF, correlations were calculated. No significant associations were found between the subjective internal state experiences and TEF (all $r s<0.32$ and $p s>0.21$ ).

\section{Discussion}

The current study assessed whether anticipated caloric content, as indicated by labels, influences metabolic responses after the consumption of equally sweet and isocaloric beverages. Based on prior findings showing that sweet taste influences the TEF, we predicted that the anticipation of higher caloric content would lead to a larger metabolic response, stronger reduction of hunger and thirst and increased fullness. However, the results did not support these hypotheses. The induced metabolic and subjective responses did not differ significantly between the three label conditions.

It was found that consuming sweetened beverages indeed induced increased metabolic responses (i.e. TEF), but the REE responses were not significantly dependent on expectations provided by different calorie content labels. This was, to the best of our knowledge, the first attempt to investigate the influence of caloric content expectations on the TEF. Based on our findings, we concluded that metabolic response is not significantly influenced by expectations of caloric content provided on labels. Our findings are not in line with previous studies that found that caloric expectations as induced by labels influence physiological responses (Cassady et al., 2012; Crum et al., 2011).

An explanation for the lack of any significant effects of the caloric labels, could be that the expectations induced by the manipulation were not strong enough. In the study of Crum and colleagues (2011) not only provided caloric information, but also included sensible versus indulgent statements, and their experimental drinks were 
advertised as a real market product. Participants were asked to observe and rate the label carefully before drinking the beverage (milkshake). Here, the attention to the label and the cover story of a more real daily life food product could have contributed to the believability of the milkshake containing different ingredients depending on label attached. Cassady and colleagues (2012) included information on the expected satiation linked to drinking the beverage. Therefore, expectations regarding satiety might have been stronger, leading to both subjective and hormonal effects. In the current study, we only provided participants with the caloric label and the general experiment information that we were interested in the effect of a sweetened low, mid- or high-caloric beverage on metabolic responses. Furthermore, a weakness of the current design was that we did not assess the extent to which participants believed the information presented on the labels.

Another possibility is that it is the congruence of sweet taste and caloric content that is critical. This possibility is consistent with a study by Veldhuizen and colleagues (2017), which found that the TEF in response to the ingestion of sweetened beverages depended on whether the sweetness of the beverage was matched to the caloric load. More specifically, TEF following consumption of an 112.5Kcal beverage was greatest when NNS was added to match the sweetness of $112.5 \mathrm{Kcal}$ sucrose compared to when too much or too little NNS was added rendering the beverage too sweet or not sweet enough for the given caloric load. Results of the current study are not in line with this match-mismatch idea either. Perhaps if we had had a very sweet beverage labeled as low calorie and a weakly sweet beverage as high calorie as compared to using matched caloric labels, we might have observed an effect, as then this mismatch effect would become more salient to influence metabolic response.

Interestingly, there was a great variability in our sample on all outcome variables. It might be that for a specific group of participants caloric content labels can influence the metabolic response in the expected direction. As an example, people scoring high on dietary restraint are more aware of the consequences of caloric intake for their health (Herman \& Polivy, 1980). Also the level of dietary restraint can influence the liking and taste experience of food (Brunstrom \& Mitchell, 2007). Additionally, in a previous study from our lab, it was found that an experimentally induced health mindset attenuated attentional bias for food only for participants scoring high on dietary restraint (Werthmann et al., 2016). Therefore, it might be valuable to include dietary restraint as a possible moderating variable in future research. 
The strength of the current study is that we used a rigorous experimental within-subjects design. Limitations include that dietary restraint was not assessed and no manipulation check was performed on the believability of the caloric content labels. Moreover, REE measurements in general are quite noisy due to slight differences between the measurements (e.g., closing of canopy and calibration artefacts) (Tenan et al., 2018).

To conclude, our study shows that labelling an isocaloric sweetened beverage as low-, mid-, or high caloric content does not significantly influence metabolic responses or subjective experiences of liking, hunger, fullness and thirst. The metabolic response was only influenced by actual intake. These findings suggest that metabolic responses are not sensitive to expectations regarding caloric content induced by labels. Future research is needed to assess whether stronger experimental manipulations of calorie content anticipations can influence metabolic responses and if this effect is moderated by the level of dietary restraint.

\section{Acknowledgements}

This study was financed by the Netherlands Organization for Scientific Research (NWO), FBC-grant (057-13-010) and the Maastricht University Interfaculty Program 'Eatwell', both awarded to Anita Jansen.

\section{Author Contributions}

S.F., A.R. and D.S. designed the study. S.F. and C.R. collected the data. S.F. analyzed the data. S.F. and A.R. wrote the manuscript, D.S., A.J. and C.R. gave feedback on the manuscript, and all authors approved the final version.

\section{Conflicts of interests}

The authors declare no conflicts of interest 


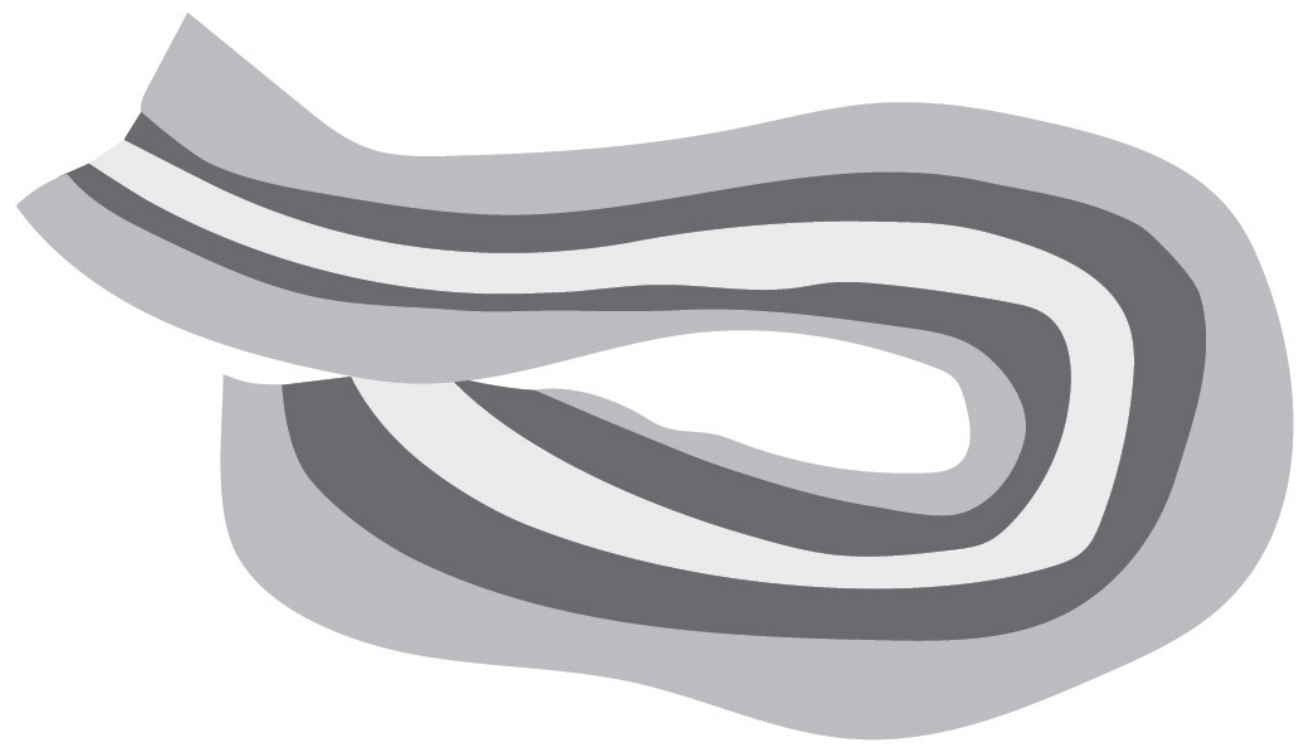




\section{CHAPTER 5}

\section{NEURAL CORRELATES OF FOOD \\ CUE EXPOSURE INTERVENTION FOR OBESITY: A CASE-SERIES $\mathrm{APPROACH}$}

Published as: Franssen, S., Jansen, A., Schyns, G., van den Akker, K., and Roefs, A. (2020). Neural Correlates of Food Cue Exposure Intervention for Obesity: A Case-Series Approach. Front. Behav. Neurosci. 14, 1-11. doi:10.3389/fnbeh.2020.00046.. 


\section{Abstract}

Background: People with overweight have stronger reactivity (e.g., subjective craving) to food cues than lean people, and this reactivity is positively associated with food intake. Cue reactivity is a learned response that can be reduced with food cue exposure therapy.

Objectives: It was hypothesized that participants after food cue exposure therapy would show reduced neural activity in brain regions related to food cue reactivity and increased neural activity in brain regions related to inhibitory control as compared to participants receiving a control-lifestyle intervention.

Method: Neural activity of ten women with overweight (BMI $\geq 27 \mathrm{~kg} / \mathrm{m}^{2}$ ) in response to individually tailored visually presented palatable high-caloric food stimuli was examined before versus after a cue exposure intervention $(n=5)$ or a control lifestyle $(n=5)$ intervention. Data were analyzed case-by-case.

Results: Neural responses to food stimuli were reduced in food-cue-reactivity-ROls after the lifestyle intervention in most participants, and generally not after the cue exposure therapy. Moreover, cue exposure did not lead to increased activity in inhibitory-control-ROls. However, decreased neural activity after cue exposure was found in most participants in the lateral occipital complex, which suggests a decreased visual salience of high-caloric food stimuli.

Conclusions: Receiving a cue exposure therapy did not lead to expected neural responses. As cue exposure relies on inhibitory learning mechanisms, differences in contexts (e.g., environments and food types) between the intervention setting and the scanning sessions may explain the general lack of effect of cue-exposure on neural activity.

\section{Keywords}

Obesity, Exposure therapy, functional MRI, case-series 


\section{Introduction}

The prevalence of obesity has reached pandemic proportions (World Health Organization, 2020). Overweight people frequently engage in weight loss attempts, but success in the long-term is rare (Bish et al., 2005; Wing \& Phelan, 2005). The main cause for obesity is a long-term energy imbalance, in which the number of consumed calories exceeds the number of expended calories for an extended time (Hall et al., 2012; Mitchell et al., 2011). Therefore, developing effective interventions to change behavior and reduce body weight is important. One possible intervention is food cue exposure therapy (CE) Jansen et al., 2016; van den Akker et al., 2016).

CE aims to reduce food cue reactivity, which is defined as appetitive responding - like increased salivation and self-reported craving - in response to food-associated cues. Food cue reactivity serves as a physiological and psychological preparation for eating (Jansen et al., 2016). Food cues can be internal, such as hunger, satiety, emotions and thoughts, but also external, such as the smell, sight and taste of food, or environmental contexts (Boswell \& Kober, 2016; Jansen et al., 2016). As compared to lean people, overweight people have a stronger food cue reactivity (Ferriday \& Brunstrom, 2011), which is related to increased food intake (Boswell \& Kober, 2016). Food cues become associated with food intake through classical conditioning (Jansen et al., 2016). As soon as food cues are reliable predictors of intake, they will elicit reactivity (Jansen et al., 2011, 2016), which in turn can lead to food intake. In CE, overweight people are repeatedly exposed to food cues while (over)eating is prevented (Bouton, 2004, 2011; Jansen et al., 2011, 2016; van den Akker, Havermans, et al., 2014). Exposure to food cues first increases food cue reactivity, but after prolonged and repeated non-reinforced exposure sessions, this reactivity decreases (Jansen et al., 2011, 2016). The CE rationale is that a new association between a food cue and intake is formed: the food cue does not predict intake. Importantly, this does not mean that the old association is unlearned (Bouton, 2004; Jansen et al., 2016). As a result of this inhibitory learning, reactivity to food cues diminishes (extinction). To optimize this inhibitory learning, maximizing 'expectancy-violation' is a key element of successful therapy (Craske et al., 2014). Expectancy violation is the reduction in a person's belief in his/her food-related expectancies (e.g., "If I feel exhausted and chocolate is available, then I will lose control and eat all chocolate). CE has been shown to be an effective method to reduce food desires and overeating (Boutelle \& Bouton, 2015; Jansen et al., 2016; Schyns et al., 2016), and it leads to short-term weight loss (Jansen et al., 2011, 2016; Schyns et al., 2016). 
To gain insight in the mechanism of change, examining neural correlates of food cue reactivity may be valuable. A recent review described the following foodcue-reactivity-related brain regions: ventral striatum with nucleus accumbens (NAcc), midbrain, orbitofrontal cortex (OFC), anterior insula (INS), gustatory cortex (GC), lateral occipital cortex (LOC) and somatosensory cortex (SSC) (Giuliani et al., 2018). An increased activity was found in these brain regions when participants viewed highcaloric foods as compared to low-caloric foods or non-food images, and this was also predictive of the amount of food consumed (Frankort et al., 2014; Giuliani et al., 2018; Hermann et al., 2019; Smeets et al., 2012; van der Laan et al., 2011). However, a meta-analysis showed that these effects were quite inconsistent: the concurrence was moderate between studies in the activated clusters to food versus non-food visual stimuli in healthy-weight participants (van der Laan et al., 2011). As CE intends to reduce food cue reactivity (Jansen et al., 2016), a decreased activity in food-cuereactivity-related brain regions is expected in the current study.

Additionally, as a candidate-mechanism behind effective CE is inhibitory learning (Boutelle \& Bouton, 2015), increased neural activity in inhibitory-control brain regions when processing palatable food stimuli is expected. Inhibitory-controlrelated brain regions include: dorso- and ventrolateral prefrontal cortex (dIPFC, vIPFC), parietal posterior cortex (PPC), dorsal anterior cingulate cortex (dACC), caudate, pre supplementary motor area (preSMA) and the globus pallidus (GP) (Giuliani et al., 2018; Kober et al., 2010).

In the current study, neural responses in food-cue-reactivity and in inhibitorycontrol brain regions to individually tailored high-caloric palatable food stimuli were examined pre- and post CE or a healthy lifestyle (LS) intervention on subject-level (i.e. for each participant separately). During a functional magnetic resonance imaging (fMRI) session, participants were instructed to actively evaluate the taste of the visually presented food stimuli (hedonic focus) or to evaluate the colors of these food stimuli (neutral focus). We hypothesized that CE, as compared to LS, would lead to reduced neural activity in food-cue-reactivity-related brain regions and increased neural activity in inhibitory-control brain regions when viewing high-caloric food stimuli, mostly in the hedonic focus condition as this focus is aligned with the experience of craving (Roefs et al., 2018). 


\section{Material and Methods}

\section{Participants}

Ten female overweight participants (BMI: $M=32.32 \mathrm{SD}=4.43 \mathrm{~kg} / \mathrm{m}^{2}$, age: $M=38.40$ SD $=10.76$ years) from a larger trial $(n=45)$ participated in this study, and were randomly assigned to CE $(n=5)$ or LS ( $n=5)$ (Schyns et al., 2019; van den Akker et al., 2016). To overcome the problem of high heterogeneity in neural responses due to individual differences that could occur in small sample sizes (Roiser et al., 2016), data were analyzed on subject-level, as separate cases (for a similar approach see: Hubacher et al., 2015). All participants, except one, were right-handed. Participants were scanned within two weeks before and within two weeks after intervention. ${ }^{8}$

Inclusion criteria included: female, age between 18 and 60 years, BMI of at least $27 \mathrm{~kg} / \mathrm{m}^{2}$, no MRI contra-indications and no history of psychiatric or neurological illnesses. The study was approved by the local Ethical Committee. The participants gave written informed consent and were compensated for participation (€ 45).

\section{Interventions}

Interventions were provided by trained students, using a strict protocol, supervised by co-author GS. Both CE and LS consisted of eight individual sessions, scheduled twice per week, during approximately one month.

During CE, participants performed several food cue exposures with a therapist. The exposure sessions were done in various overeating contexts (e.g. at the laboratory, at home watching television or work,). Additionally, participants performed daily exposure exercises on their own at home or at other overeating-associated environmental contexts. LS consisted of four face-to-face sessions alternated with four telephone sessions. LS participants received healthy lifestyle advice, performed mindfulness and power posing exercises, and obtained psychoeducation on body image. For this intervention, also daily homework exercises were given on mindfulness and on previous therapy session content. For a detailed description of both interventions see (van den Akker et al., 2016).

\footnotetext{
${ }^{8}$ Note that these pre-intervention fMRI data was also included in a previous study (Franssen S, Jansen A, van den Hurk J, et al. Power of mind: attentional focus rather than food palatability dominates neural responding to visual food stimuli in females with overweight, manuscript under revision)
} 


\section{Behavioral assessments}

\section{BMI measurement}

Weight and height were measured pre and post-intervention to compute BMI in kg/ $\mathrm{m}^{2}$.

\section{Hunger assessment}

Participants were asked to refrain from eating or drinking (except water) for at least one hour before the scan-sessions. To check compliance and have an indicative for subjective hunger, self-reported hunger was measured using a 100-mm visual analogue scale (VAS), with the question: "How hungry do you feel at this moment?" ranging from 0 (not hungry at all) to 100 (very hungry) at the start of each session. Additionally, participants registered what and at what time they had eaten last.

\section{Expectancy violation}

Eight food-cue-associated eating beliefs were rated on perceived expectancy if an associated cue would be followed by eating. Expectancies were measured pre- and post-intervention using $100 \mathrm{~mm}$ VAS, with a higher score reflecting a greater perceived expectancy of eating (see methodology paper for details: (van den Akker et al., 2016)).

\section{Stimuli}

\section{Individual stimulus selection}

Food stimuli used in the fMRI experiment were individually tailored. Each participant selected their five most palatable food items from a list of 33 high-caloric food items in an online questionnaire that was completed approximately one week before the first scanning session. She then rated the selected stimuli on 10-point scales ranging from 1 (not palatable at all) to 10 (very palatable).

\section{Stimulus presentation}

For each of the five chosen palatable food items, two different pictures were included in the fMRI stimulation protocol, to avoid visual adaptation by seeing the same picture too often. Pictures were presented as pop-out high-resolution coloured images on a 
light grey background (RGB: 191191 191; CKYM: 252020 0) in the centre of a black screen covering a visual angle of approximately $12^{\circ}$.

\section{Experimental task}

\section{Attentional focus manipulation}

The participant performed a fast-paced 1-back task in each functional run to induce an attentional focus (hedonic versus neutral). During the 1-back task, the participant compared each presented food picture (starting from the $2^{\text {nd }}$ presented picture) to the previously presented picture, and indicated whether the presented food was more or less palatable than the previous one (hedonic focus), or whether the picture contained more or fewer colors than the previous one (neutral focus). Each food stimulus was presented for 500 ms, with an inter-stimulus interval (ISI) as a response window of 1500 ms. The participant's responses were registered using a buttonbox, with a right index finger press for "fewer" and a right middle finger press for "more".

\section{fMRI Stimulation protocol}

The fMRI task consisted of four runs. In each run, six different conditions were presented, but for the current study only two conditions were relevant and included in the analyses: blocks with palatable high-caloric food stimuli - neutral focus (PALNEU) and blocks with palatable high-caloric food stimuli - hedonic focus (PAL-HED). Ten blocks were presented seven times in a randomized order with 12 stimuli each, across the four functional runs. Prior to each block, a cue-word "taste" or "color" was presented for 1 second to inform the participant which attentional focus to apply. Blocks lasted 24 seconds and were always followed by a 20 second rest block (fixation cross). Total functional scanning time was approximately 35 minutes.

\section{MRI data acquisition}

Images were acquired on a 3 Tesla MRI scanner (Magnetom Prisma Fit, Siemens Medical Systems) using a 64-channel head/neck coil. Functional ( $T_{2}{ }^{*}$-weighted) images were acquired using multiband gradient echo-planar imaging in an axial interleaved order (Feinberg et al., 2010) with the following settings: TR = $2000 \mathrm{~ms}$, TE = 30 ms, flip angle $=77^{\circ}, F O V=208 \times 124 \mathrm{~mm}^{2}$, and voxel size of $2 \times 2 \times 2 \mathrm{~mm}^{3}$. To ensure whole brain coverage, slices were acquired in a backward tilted direction of approximately 
15 degrees to the transversal - coronal line. As anatomical scan, a high-resolution, three-dimensional (3D) $T_{1}$-weighted MPRAGE scan was acquired, with the following settings: TR $=2250 \mathrm{~ms}, \mathrm{TE}=2.21 \mathrm{~ms}$, flip angle $=9^{\circ}, \mathrm{FOV}=256 \times 192 \mathrm{~mm}^{2}$, and voxel size $1 \times 1 \times 1 \mathrm{~mm}^{3}$ and had a duration of \pm 5 minutes.

\section{fMRI data analysis}

\section{Preprocessing}

Analyses were performed using SPM12 (Statistical Parametric Mapping, London, UK) and Matlab version 8.3.0.532 (R2014a). Functional images were slice-time corrected, realigned, co-registered, normalized using unified brain segmentation, and spatially smoothed using a Gaussian kernel of $6 \mathrm{~mm}$ full width at half-maximum (FWHM). Preprocessed functional volume time series were used for statistical analysis.

\section{Statistical analysis}

To compare the session differences on subject-level, a general linear model (GLM) design matrix was created including the two scan-sessions (pre- and postintervention) as eight consecutive runs. Each experimental task condition was set as a predictor, which resulted in six predictors of interest per run (with two of interest for the current study). Additionally, six motion and eight run mean intensity predictors of no interest were added to the model as confound regressors. Predictor time courses were obtained using condition box-car shaped waves convolved with a two-gamma ideal hemodynamic response function (HRF).

\section{Case series approach: first-level analysis}

To investigate the effects of interest, we computed the following contrasts on subject-level for the high-caloric palatable food conditions: (1) main effect of session ( $t$-contrasts A: pre-intervention > post-intervention and B: post-intervention > preintervention,) and (2) session (pre-intervention versus post-intervention) * attentional focus (neutral versus hedonic) interaction F-contrasts. To extract beta values, each condition of interest was also contrasted against baseline. ${ }^{9}$

\footnotetext{
${ }^{9}$ For participant 2 of the LS group only 3 runs were acquired in the pre-intervention session due to set-up problems. Therefore, also only 3 runs of the post-intervention session were included for this participant. This was done to be able to balance the conditions in the GLM for defining contrasts. For the post-intervention session, we selected the 3 runs with best data quality (i.e., least movement of participant during scanning).
} 


\section{Region of interest (ROI) analysis}

We defined a priori ROI masks for food cue reactivity based on the review of Giuliani and colleagues (Giuliani et al., 2018), including: ventral striatum with NAcc, midbrain, OFC, anterior INS, GC, LOC and SSC and for inhibitory control, including: dIPFC, VIPFC, PPC, dACC, caudate, preSMA and the GP. The ROI masks were manually generated by using the WFU Pickatlas tool (version 3.0.5) in SPM12.

To correct for multiple comparisons, family-wise error (FWE) correction based on Gaussian random field theory was applied to control for false positives at $a=0.05$ on subject-level (Eklund et al., 2016). This method was applied for the statistical maps of the main effects of session, and was combined with a clustersize threshold ( $k$ ) of three contiguous voxels to only include more robust clusters. For the subtler session * attentional focus interaction, uncorrected statistical maps with $p<0.001$ with $\mathrm{k}=$ 3 voxels were reported. The MarsBar toolbox (http://marsbar.sourceforge.net/) was used to extract beta values in SPM12. For localization and clustersize information of activated clusters, XJview (http://www.alivelearn.net/xjview/) was used.

\section{Results}

\section{Behavioral assessments}

An overview of behavioral assessments is provided in Table 1. The time between first and second scan session ranged for the cue-exposure condition 34-43 days and for the lifestyle intervention 36-58 days. Three CE and four LS participants lost weight after the intervention. Hunger-ratings were higher post-intervention than preintervention in all CE participants, whereas this was only true for two LS-participants (LS1 \& LS5). However, seven of the ten participants reported relatively low hunger at post-intervention (scores $\leq 44$ on a 0 - 100 VAS). All participants rated their selected foods as highly palatable (average scores $\geq 8.8$ on 10-point scale). Expectancy violation changes (post-intervention - pre-intervention) differed between CE and LS participants. All CE participants showed a higher reduction of eating expectancies after intervention as compared to the LS participants. 


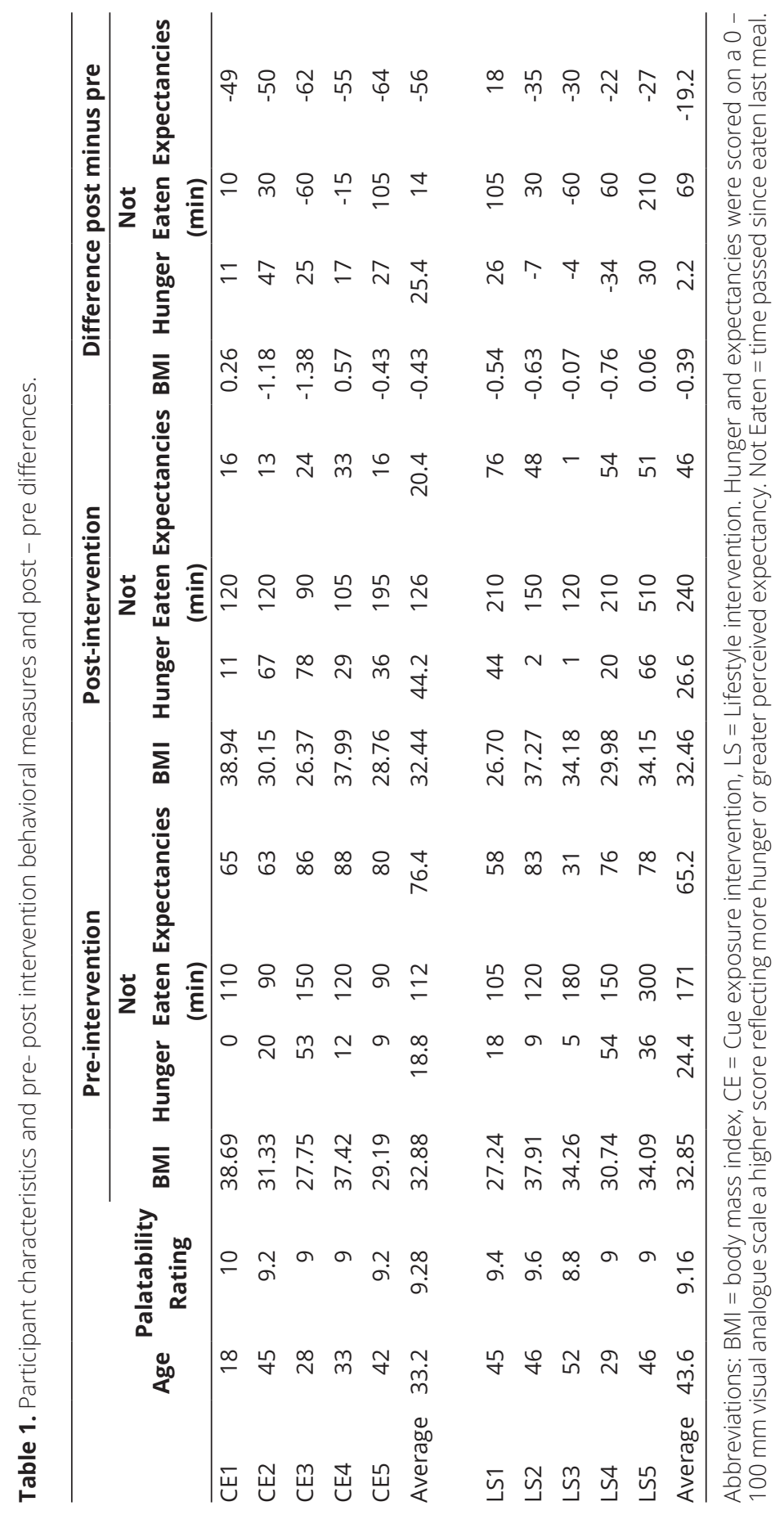




\section{Neural responses}

In Figures 1 and 2, main effects of session are displayed, as well as the session * attentional focus interaction per participant in the food-cue-reactivity-ROIs. Details of each significant cluster can be found in supplementary Tables 2 and 3.

\section{Main effects session}

Contrary to our hypothesis, food-cue-reactivity-related activity was not substantially reduced after intervention for CE participants. In fact, LS participants showed reductions in more ROIs (e.g., SSC, INS, LOC \& OFC; see participants LS1 \& LS3), and involved clusters were larger. However, reduction in beta values was larger in those clusters that changed significantly from pre- to post-intervention in CE participants. Note that these clusters were substantially smaller and localized in the LOC solely (see participants: CE1, CE3, CE4 and CE5). To examine opposite effects, we also compared post-intervention > pre-intervention contrasts. Here, unexpectedly, the CE participants showed also increased activation in small clusters in the LOC (see: CE1 and CE5) and in the OFC (CE2) after intervention. LS participants showed an increase in activity in the SSC (LS2, LS4 and LS5) and in the LOC (LS3).

\section{Session * attentional focus interaction}

As with the main effect of session, the interaction effect was also mainly observed in LS participants. Analyses of the interaction yielded significant clusters for all LS participants. Here, four LS participants (LS1, LS3, LS4, and LS5) showed a larger reduction from pre to post with the hedonic focus than with the neutral focus in the right INS and OFC and bilaterally in the SSC and LOC. For participant LS2 this was reversed, activity in food-cue-reactivity-ROIs (right OFC and left SSC) was increased from pre to post with the hedonic focus. Two CE participants showed an interaction effect, where participant CE4 showed a more reduced activation pre to post in the hedonic focus than in the neutral focus in a very small cluster in the right SCC. Participant CE3 showed more robust clusters, involving the INS and the SCC with an unexpectedly larger reduction in activation from pre to post in the neutral focus condition than the hedonic focus.

We also compared neural activity per participant in inhibitory-control-ROls. Tests of the main- and interaction-effects did not lead to any meaningful results for 
the CE intervention either. The clusters of neural activity per participant can be found in supplementary Tables 1 and 2

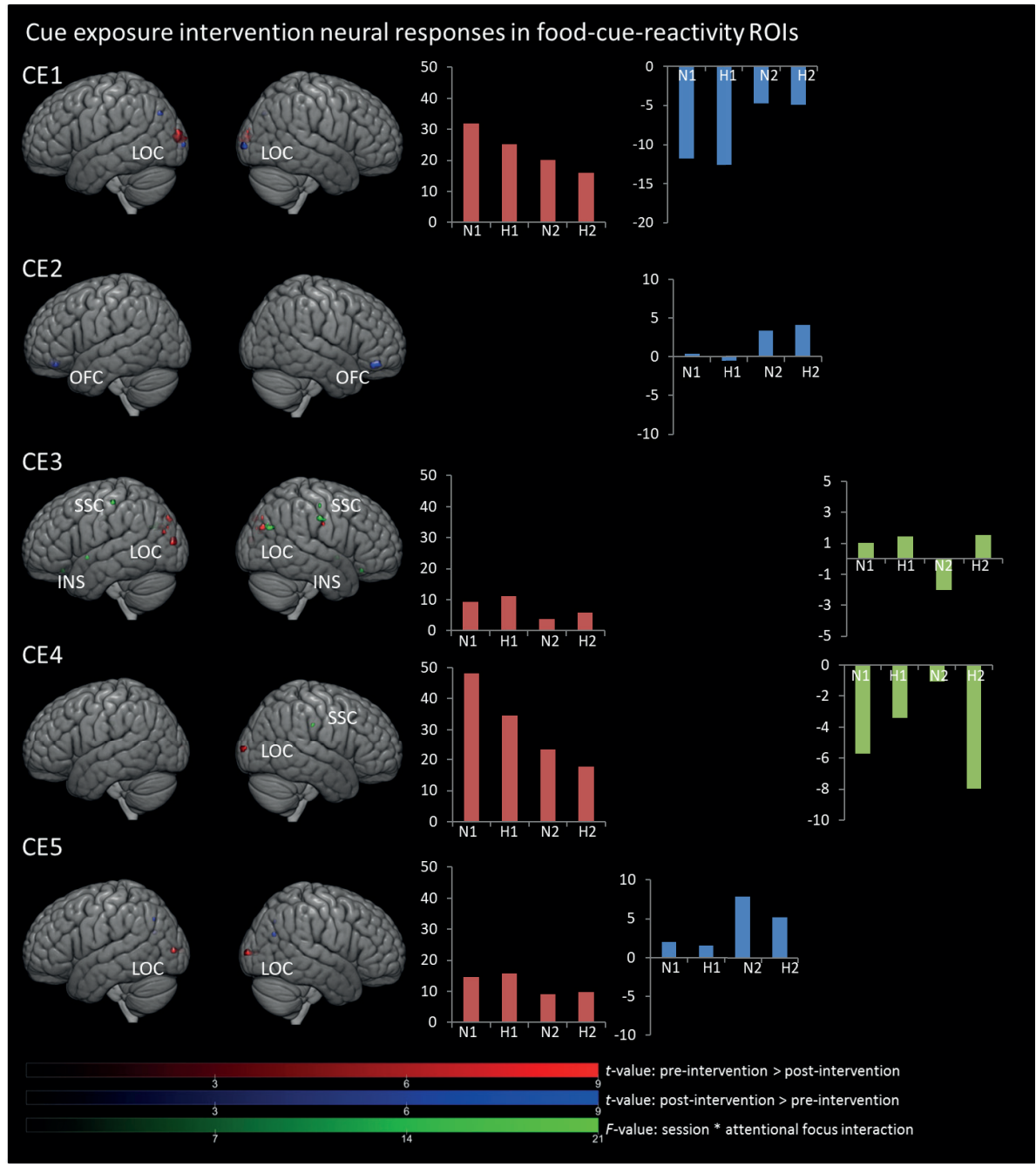

Figure 1. Results from univariate analyses per participant for cue exposure intervention in foodcue-reactivity-ROls. t-maps of significant main effects of session are shown in food-cue-reactivityROls: pre $>$ post intervention in red, post $>$ pre intervention in blue $(p<0.05$ FWE cor) and F-map of session * attentional focus interaction ( $p<0.001$ unc.) in green. Bar plots represents mean extracted beta values from the contributing clusters per condition per comparison. N1 = neutral attentional focus pre intervention, $\mathrm{H} 1=$ hedonic attentional focus pre intervention, N2 = neutral attentional focus post intervention, $\mathrm{H} 2$ = hedonic attentional focus post intervention, LOC = Lateral Occipital Complex, SSC = somatosensory cortex, OFC= orbitofrontal cortex, INS $=$ Insula 
Table 2. Significant clusters from univariate analyses per participant for cue exposure intervention in food-cue-reactivity-ROIs.

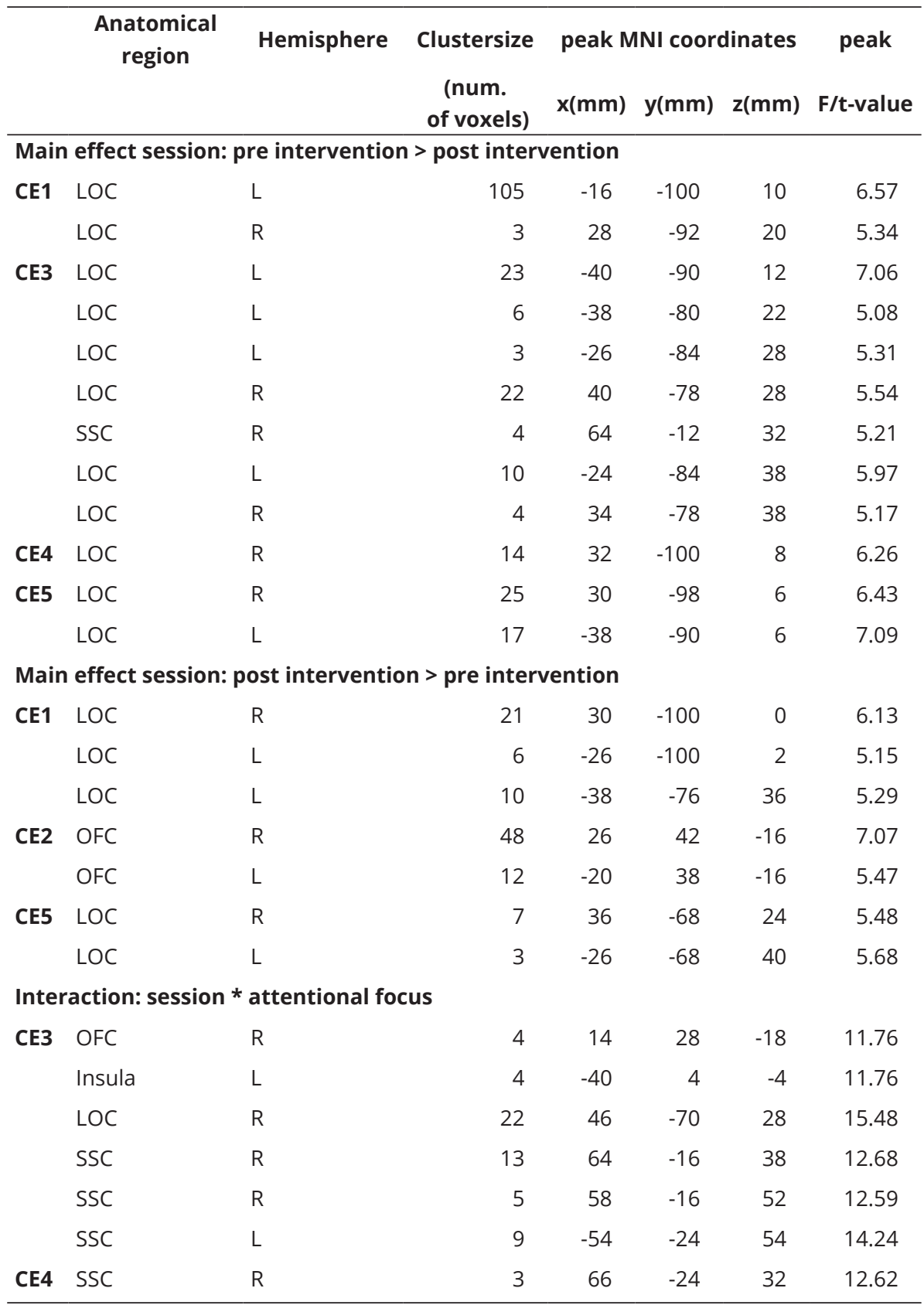

Abbreviations: $\mathrm{L}=$ left, $\mathrm{R}=$ right, $\mathrm{MNI}=$ Montreal Neurological Institute, $\mathrm{LOC}=$ Lateral Occipital Complex, SSC = somatosensory cortex, OFC= orbitofrontal cortex 


\section{Healthy lifestyle intervention neural responses in food-cue-reactivity ROIs}
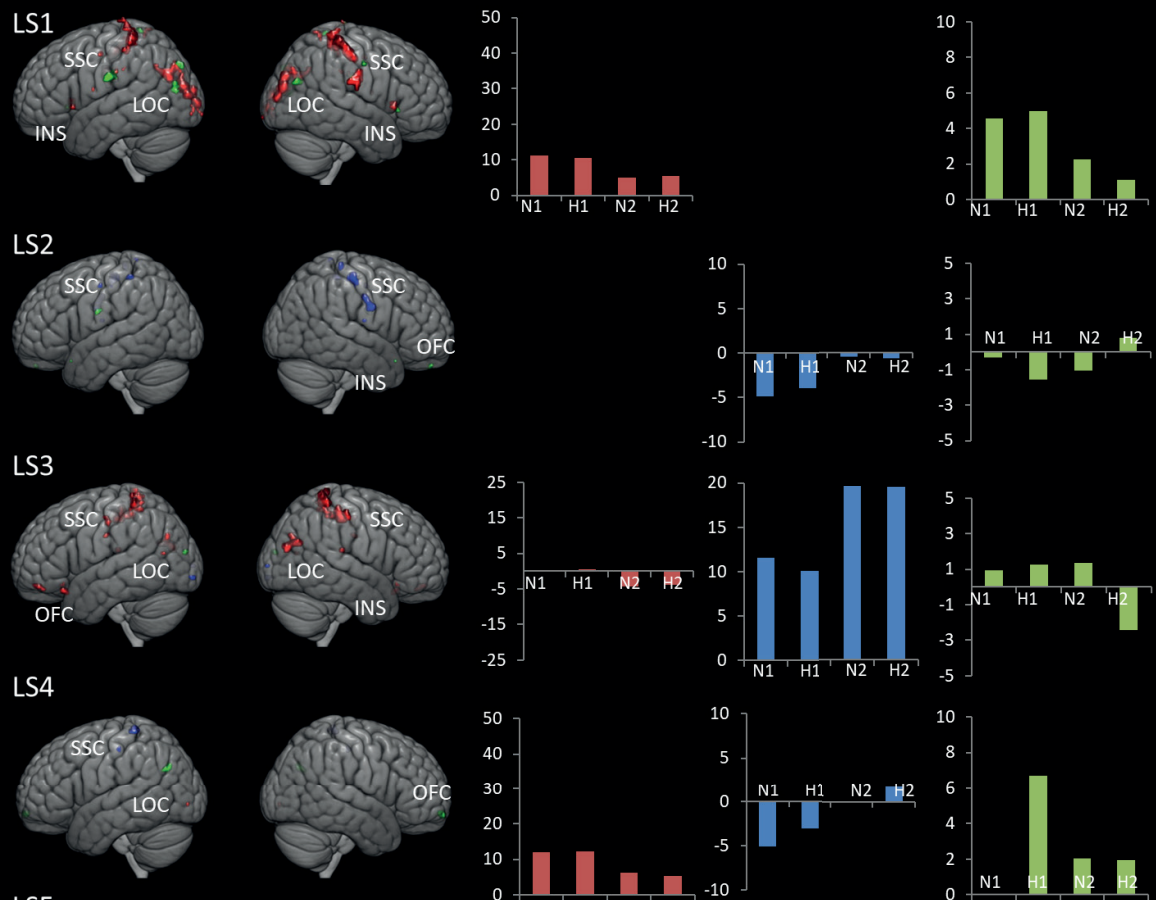

LS5
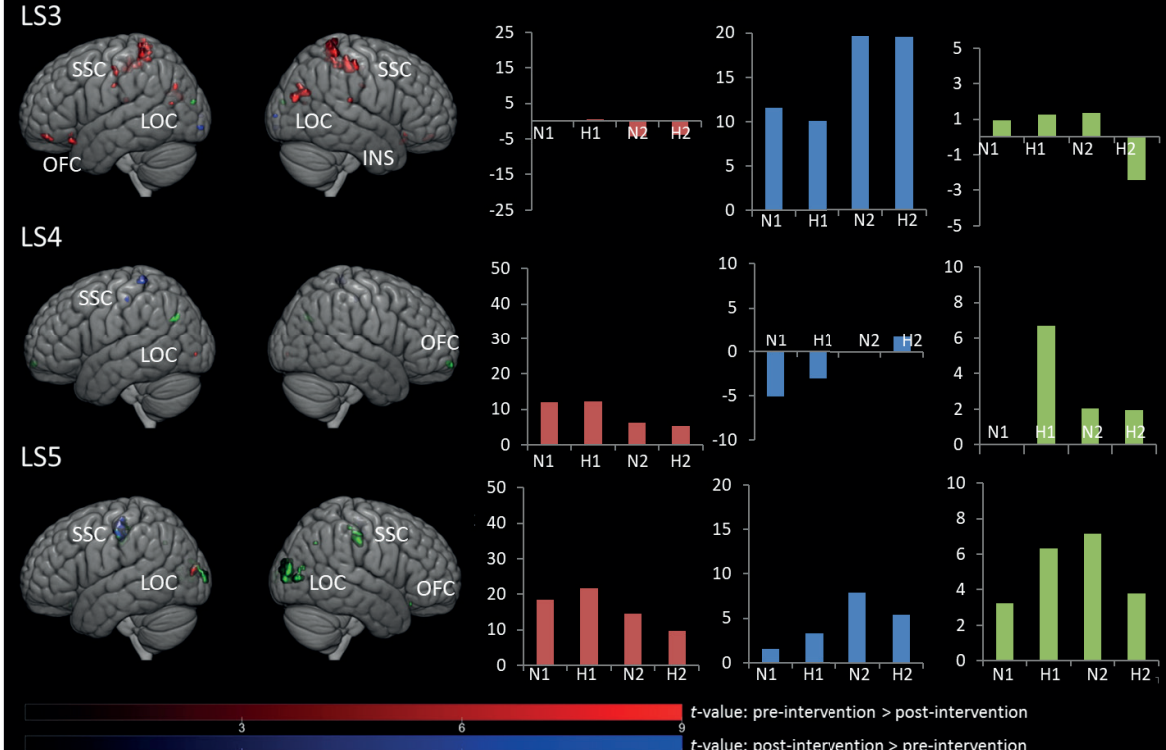

$\begin{array}{ll}3 & 6 \\ 3 & 6\end{array}$

t-value: pre-intervention > post-interventio

$t$-value: post-intervention > pre-intervention

${ }_{21} F$-value: session * attentional focus interaction

Figure 2. Results from univariate analyses per participant for healthy lifestyle intervention in food-cue-reactivity-ROIs. t-maps of significant main effects of session are shown in food-cuereactivity-ROIs: pre $>$ post intervention in red, post $>$ pre intervention in blue $(p<0.05$ FWE cor) and F-map of session * attentional focus interaction ( $p<0.001$ unc.) in green. Bar plots represents mean extracted beta values from the contributing clusters per condition per comparison. $\mathrm{N} 1$ = neutral attentional focus pre intervention, $\mathrm{H} 1$ = hedonic attentional focus pre intervention, $\mathrm{N} 2$ = neutral attentional focus post intervention, $\mathrm{H} 2$ = hedonic attentional focus post intervention, LOC = Lateral Occipital Complex, SSC = somatosensory cortex, OFC= orbitofrontal cortex, INS = Insula 
Table 3. Significant clusters from univariate analyses per participant for healthy lifestyle intervention in food-cue-reactivity-ROIs.

\begin{tabular}{|c|c|c|c|c|c|c|c|}
\hline & Anatomical region & Hemisphere & $\begin{array}{l}\text { Clustersize } \\
\text { (num. } \\
\text { of voxels) }\end{array}$ & $\begin{array}{l}\text { peak } N \\
x(\mathrm{~mm})\end{array}$ & $y(m m)$ & $\mathrm{z}(\mathrm{mm})$ & $\begin{array}{c}\text { peak } \\
\text { F/t-value }\end{array}$ \\
\hline \multicolumn{8}{|c|}{ Main effect session: pre intervention > post intervention } \\
\hline \multirow[t]{21}{*}{ LS1 } & SSC & $\mathrm{R}$ & 288 & 50 & -28 & 58 & 8.19 \\
\hline & LOC & L & 271 & -26 & -86 & 20 & 11.32 \\
\hline & LOC & $\mathrm{R}$ & 188 & 28 & -88 & 20 & 9.04 \\
\hline & SSC & L & 156 & -40 & -40 & 68 & 9.75 \\
\hline & SSC & $\mathrm{R}$ & 79 & 68 & -18 & 24 & 8.11 \\
\hline & LOC & L & 55 & -20 & -100 & 2 & 9.03 \\
\hline & LOC & L & 34 & -18 & -96 & 14 & 11.49 \\
\hline & Insula & $\mathrm{R}$ & 18 & 40 & 18 & 4 & 6.08 \\
\hline & SSC & $\mathrm{R}$ & 17 & 12 & -56 & 72 & 6.57 \\
\hline & LOC & $\mathrm{R}$ & 14 & 38 & -74 & 34 & 5.94 \\
\hline & SSC & L & 14 & -22 & -30 & 82 & 7.00 \\
\hline & Insula & L & 9 & -40 & 18 & -2 & 6.08 \\
\hline & LOC & L & 8 & -32 & -95 & -8 & 5.86 \\
\hline & SSC & L & 8 & -56 & -18 & 26 & 5.15 \\
\hline & SSC & L & 8 & -4 & -8 & 48 & 5.96 \\
\hline & SSC & L & 8 & -54 & -32 & 52 & 6.61 \\
\hline & SSC & L & 6 & -58 & -24 & 32 & 5.93 \\
\hline & LOC & L & 4 & -16 & -104 & -8 & 9.40 \\
\hline & LOC & L & 3 & -10 & -96 & 0 & 5.72 \\
\hline & SSC & L & 3 & -54 & -2 & 16 & 5.07 \\
\hline & SSC & L & 3 & -44 & -32 & 36 & 5.13 \\
\hline \multirow[t]{9}{*}{ LS3 } & SCC & $\mathrm{R}$ & 200 & 32 & -46 & 70 & 9.22 \\
\hline & LOC & $\mathrm{R}$ & 109 & 44 & -72 & 24 & 6.43 \\
\hline & SSC & L & 105 & -28 & -48 & 68 & 8.51 \\
\hline & SSC & $\mathrm{R}$ & 78 & 34 & -28 & 54 & 7.72 \\
\hline & OFC & L & 24 & -34 & 48 & 16 & 6.44 \\
\hline & SSC & $\mathrm{R}$ & 24 & 54 & -24 & 54 & 6.11 \\
\hline & SSC & L & 20 & -56 & -18 & 44 & 5.64 \\
\hline & OFC & L & 19 & -32 & 22 & -20 & 6.56 \\
\hline & SSC & L & 19 & -18 & -46 & 58 & 5.98 \\
\hline
\end{tabular}


Table 3 (Continued)

\begin{tabular}{|c|c|c|c|c|c|c|c|}
\hline & Anatomical region & Hemisphere & $\begin{array}{c}\text { Clustersize } \\
\text { (num. } \\
\text { of voxels) }\end{array}$ & $\begin{array}{l}\text { peak } M \\
x(m m)\end{array}$ & $y(m m)$ & $z(\mathrm{~mm})$ & $\begin{array}{c}\text { peak } \\
\text { F/t-value }\end{array}$ \\
\hline & SSC & $\mathrm{R}$ & 14 & 6 & -42 & 62 & 7.25 \\
\hline & SSC & $\mathrm{R}$ & 14 & 8 & -42 & 74 & 7.72 \\
\hline & SSC & $\mathrm{R}$ & 11 & 6 & -36 & 50 & 6.19 \\
\hline & LOC & L & 10 & -40 & -72 & 32 & 5.40 \\
\hline & Insula & $\mathrm{R}$ & 8 & 34 & -26 & 20 & 5.43 \\
\hline & SSC & L & 7 & -40 & -16 & 34 & 5.64 \\
\hline & SSC & L & 6 & -4 & -48 & 62 & 5.68 \\
\hline & SSC & L & 4 & -14 & -40 & 54 & 5.55 \\
\hline & SSC & L & 4 & -14 & -44 & 74 & 6.80 \\
\hline & OFC & L & 3 & -34 & 24 & -12 & 5.29 \\
\hline & LOC & L & 3 & -42 & -68 & 16 & 5.67 \\
\hline LS4 & LOC & L & 3 & -32 & -88 & -4 & 5.06 \\
\hline LS5 & LOC & L & 43 & -28 & -90 & 14 & 6.90 \\
\hline \multicolumn{8}{|c|}{ Main effect session: post intervention > pre intervention } \\
\hline \multirow[t]{8}{*}{ LS2 } & SSC & $\mathrm{R}$ & 64 & 56 & -2 & -28 & 5.79 \\
\hline & SSC & $\mathrm{R}$ & 37 & 42 & -22 & 58 & 6.81 \\
\hline & SSC & L & 11 & -32 & -38 & 58 & 5.29 \\
\hline & SSC & $\mathrm{R}$ & 11 & 36 & -32 & 68 & 5.67 \\
\hline & SSC & L & 5 & 28 & -26 & -56 & 5.27 \\
\hline & SSC & $\mathrm{R}$ & 4 & 28 & -26 & 56 & 5.19 \\
\hline & Insula & $\mathrm{R}$ & 3 & 40 & -12 & 16 & 5.12 \\
\hline & SSC & L & 3 & -4 & -44 & 72 & 5.26 \\
\hline \multirow[t]{2}{*}{ LS3 } & LOC & L & 6 & -28 & -98 & -8 & 6.42 \\
\hline & LOC & $\mathrm{R}$ & 4 & 24 & 98 & 4 & 5.07 \\
\hline \multirow[t]{2}{*}{ LS4 } & SSC & L & 33 & -34 & -36 & 68 & 5.51 \\
\hline & SSC & L & 4 & -52 & -24 & 50 & 5.31 \\
\hline LS5 & SSC & L & 130 & -50 & -22 & 44 & 6.55 \\
\hline \multicolumn{8}{|c|}{ Interaction: session * attentional focus } \\
\hline \multirow[t]{4}{*}{ LS1 } & SSC & L & 63 & -66 & -16 & 26 & 29.22 \\
\hline & LOC & L & 26 & -46 & -78 & 22 & 15.85 \\
\hline & LOC & $\mathrm{R}$ & 20 & 50 & -72 & 26 & 19.03 \\
\hline & LOC & L & 18 & -26 & -84 & 40 & 14.10 \\
\hline
\end{tabular}


Table 3 (Continued)

\begin{tabular}{|c|c|c|c|c|c|c|c|}
\hline & Anatomical region & Hemisphere & $\begin{array}{c}\text { Clustersize } \\
\text { (num. } \\
\text { of voxels) }\end{array}$ & $\begin{array}{l}\text { peak } M \\
x(\mathrm{~mm})\end{array}$ & $y(m m)$ & $\mathrm{z}(\mathrm{mm})$ & $\begin{array}{c}\text { peak } \\
\text { F/t-value }\end{array}$ \\
\hline & SSC & $L$ & 8 & -4 & -46 & 70 & 23.43 \\
\hline & SSC & $\mathrm{R}$ & 6 & 60 & -10 & 44 & 15.61 \\
\hline & Insula & $\mathrm{R}$ & 4 & 34 & 22 & 0 & 13.08 \\
\hline & SSC & L & 3 & -18 & -26 & 80 & 15.45 \\
\hline \multirow[t]{3}{*}{ LS2 } & SSC & L & 9 & -62 & -6 & 24 & 12.60 \\
\hline & OFC & $\mathrm{R}$ & 4 & 6 & 52 & -26 & 12.74 \\
\hline & OFC & $\mathrm{R}$ & 3 & 14 & 18 & -22 & 13.18 \\
\hline LS3 & LOC & L & 7 & -18 & -90 & 18 & 12.87 \\
\hline \multirow[t]{2}{*}{ LS4 } & OFC & $\mathrm{R}$ & 18 & 18 & 64 & -12 & 13.21 \\
\hline & LOC & L & 17 & -34 & -70 & 34 & 12.15 \\
\hline \multirow[t]{10}{*}{ LS5 } & LOC & $\mathrm{R}$ & 224 & 36 & -90 & 12 & 21.36 \\
\hline & SSC & $\mathrm{R}$ & 68 & 48 & -22 & 38 & 22.93 \\
\hline & LOC & L & 47 & -26 & -100 & 10 & 15.41 \\
\hline & LOC & L & 35 & 52 & -78 & 6 & 16.79 \\
\hline & SSC & L & 18 & -38 & -24 & 56 & 13.19 \\
\hline & SSC & L & 17 & -52 & -20 & 44 & 13.48 \\
\hline & LOC & $\mathrm{R}$ & 8 & 38 & -76 & 14 & 11.73 \\
\hline & LOC & $\mathrm{R}$ & 4 & 32 & -62 & 38 & 12.11 \\
\hline & SSC & $\mathrm{R}$ & 4 & 50 & -32 & 52 & 12.65 \\
\hline & OFC & $\mathrm{R}$ & 3 & 30 & 26 & -18 & 11.06 \\
\hline
\end{tabular}

Abbreviations: $\mathrm{L}=$ left, $\mathrm{R}=$ right, $\mathrm{MNI}=$ Montreal Neurological Institute, $\mathrm{LOC}=$ Lateral Occipital Complex, SSC = somatosensory cortex, OFC= orbitofrontal cortex 


\section{Discussion}

Contrary to our hypothesis, the results showed that for these cases a cue exposure intervention did not lead to a significantly stronger reduction in neural activity in food-cue-reactivity-ROls, in response to visual high-caloric palatable food stimuli, as compared to the participants that received a lifestyle intervention. In fact, most participants' reductions in neural activity in food-cue-reactivity-ROls were more pronounced and more widespread after a lifestyle intervention and mostly with a hedonic attentional focus. When comparing inhibitory-control-ROIs on subject-level, no meaningful results were observed.

Surprisingly, the expected reduced activity was more apparent in LS participants (in e.g. SSC, INS, OFC and LOC). During the intervention, LS participants received education on dieting and healthy weight loss and on nutrients and energy balance (van den Akker et al., 2016). This could have raised awareness of negative health aspects of high caloric foods, which may have contributed to participants' reduced neural responses to high-caloric foods. This interpretation aligns well with previous studies, showing that focusing on negative health aspects can control reward-related activity to visually presented high-caloric food stimuli (Hollmann et al., 2012; Siep et al., 2012). Although participants were instructed during scanning to attend to the hedonic aspects of the foods presented, this lifestyle training may have interfered with this hedonic focus during the post-intervention scanning-session by increasing awareness of negative health aspects.

Unexpectedly, CE did not lead to a significant reduction of neural activity in most cases in food-cue-reactivity-ROls. Behavioral outcomes showed that selfreported expectancy violations did improve specifically for the CE participants. Also, hunger was higher for all CE participants at post-intervention measurement. However, these CE-related behavioral effects could not be meaningfully related to post-pre intervention patterns of neural activity. These neural pre-post intervention findings could be the consequence of participants learning a new inhibitory association (the cue does not predict intake) during food cue exposure, which then exists next to the original disinhibiting association (the cue does predicts intake) (Jansen et al., 2016). That is, the food-cue-intake association is not erased, and therefore food cues might still trigger neural activity in food-cue-reactivity-ROls. However, also in the inhibitorycontrol-ROls, no strong increased neural activity in CE participants was found after intervention in these inhibitory-control regions. 
Important to realize is that inhibitory learning during extinction is contextdependent and food-specific (Bouton, 2004, 2011; Jansen et al., 2016). Both the context (fMRI scanner versus a laboratory room, participants' home and other relevant contexts) and the food stimuli differed between the current fMRI measurement and the intervention setting. Furthermore, a CE intervention only led to reduced consumption of the exposed foods, but not of other foods (Schyns et al., 2016, 2018, 2019). So, there was no generalization to other foods. In an earlier study (Frankort et al., 2014), we did observe a reduction in neural activity in food-cue-reactivity-ROls after cue exposure. Importantly, here, the cue exposure and measurement of neural activity both took place in the scanner while using the same food stimuli throughout (i.e., chocolate) (Frankort et al., 2014). Taken together, these findings underline the importance of considering context and the food-specificity of cue exposure while examining neural responses.-

In line with our hypothesis, the current study showed reduced activation in the lateral occipital complex (LOC) in four participants after CE. The LOC was identified, in a meta-analysis comparing visual food to non-food stimuli, as one of the main brain regions involved in visual food cue processing (van der Laan et al., 2011). The decreased LOC activity may reflect a decrease in visual saliency of the palatable highcaloric foods as a result of CE. As this decreased LOC activation was specifically found for the CE participants, it therefore might be a precursor for extinction.

A limitation of this study is that due to the inclusion of only female participants and the case-series analyses approach, it is hard to translate the current results to a group-intervention effect, or to a broader population (i.e. males). Results should be interpreted with caution. Data was analyzed with a-priori defined ROI masks, which reduces the between-subjects variability of activation locations and makes interpreting and comparing findings more reliable. The scanning-protocol pre- and post-intervention was kept exactly the same, which made it a strong within-subject design, and therefore the current study might give interesting leads for conducting a group-level future study. Future research needs to replicate these findings, and investigate whether neural changes induced by a lifestyle intervention are related to concurrent and future weight change.

\section{Disclosure Statement}

The authors have no conflicts of interest to declare. 


\section{Funding sources}

This study was partly financed by the Netherlands Organization for Scientific Research (NWO) vici-grant (453.10.006) and the Maastricht University Interfaculty Program 'Eatwell' awarded to Anita Jansen, and NWO vidi-grant (452.16.007) awarded to Anne Roefs.

\section{Author Contributions}

S.F. A.R. and A.J. designed the study. S.F. collected the data. G.S. and K.A. designedand coordinated the interventions. S.F. analyzed the data, S.F. and A.R. wrote the manuscript, A.J., G.S. and K.A. gave feedback on the manuscript, and all authors approved the final version. 


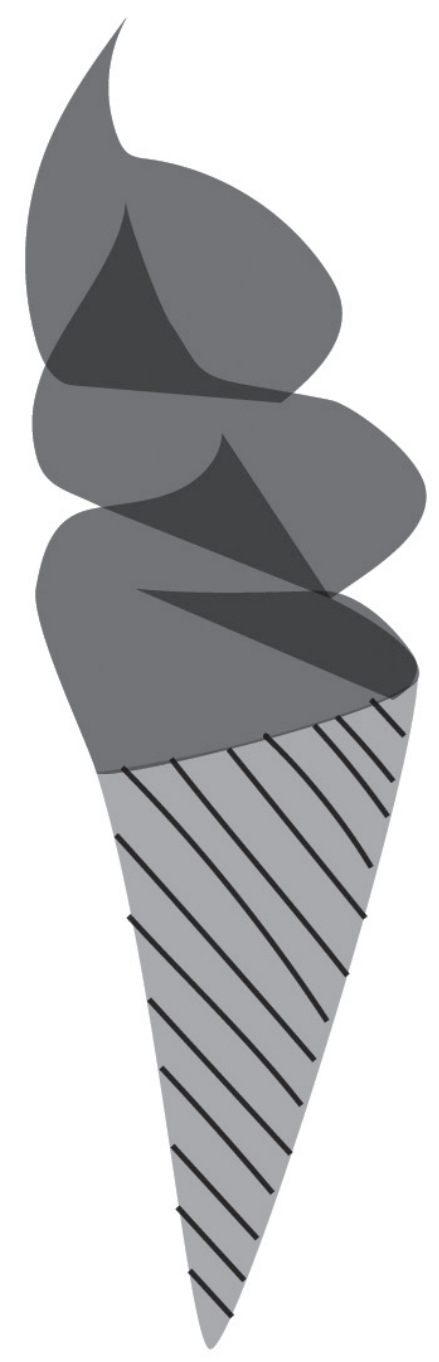


CHAPTER 6

$$
\begin{array}{r}
\text { SUMMARY OF MAIN FINDINGS AND } \\
\text { GENERAL DISCUSSION }
\end{array}
$$


The way people react to food cues is not always the same, and it may depend on someone's current mindset. Such mindsets can differ within and between people and might influence eating behavior. Therefore, the aim of the current dissertation was to examine the influence of mindset on psychological and physiological variables involved in eating behavior. Mindset was operationalized in this dissertation as: loss of control versus control mindset, hedonic attentional focus versus neutral attentional focus, and high-versus mid- versus low-caloric label. The effects of these mindsets were assessed on different dependent variables: food intake in chapter 3; subjective food experiences (e.g. self-control, craving, liking, hunger, satiety) in chapter 3 and 4; neural responses (activity in mesocorticolimbic system and control related brain areas) in chapter 2, 3 and 5; hunger and satiety related gut-hormones (respectively: ghrelin and glucagon-like peptide 1; GLP-1) in chapter 3; and metabolism (by calculating the thermic effect of food from resting energy expenditure) in chapter 4 . In addition, we examined the effects of food cue exposure therapy on neural responses to food in

\section{chapter 5.}

The general hypothesis was that a loss of control mindset or hedonic focus and a high caloric label on food - as compared to a control mindset or health focus and a low caloric label - would lead to reward and craving related bodily and subjective responding. That is, increased neural responses in the mesocorticolimbic system, an increase in the hunger hormone ghrelin, an increase in the metabolic thermic effect of food, and increases in subjective reports of craving, hunger and actual intake. Also, a decrease was expected in control-related neural responses and the satiety hormone GLP-1. Furthermore, it was hypothesized that repeated sessions of food cue exposure therapy would reduce neural activity in food-cue-reactivityrelated brain regions and increased neural activity in control-related brain regions when viewing high-caloric food stimuli, and more strongly when in a hedonic focus condition. Below the main findings are summarized and discussed in more detail. This chapter is concluded by discussing the theoretical implications and future directions.

\section{Food intake}

In chapter 3, the influence of mindset on actual food intake was measured using a bogus taste test. Here, a loss of control and a control mindset were induced by having chocolate lovers view short movies on either loss-of-control or control. It was shown that these mindsets influenced food intake in the expected direction. That is, 
people ate more chocolate after an induced loss of control mindset as compared to the control mindset. Previous studies showed that beliefs generated by health claims or caloric content labels of food products influence food intake (e.g., McCann et al., 2013; Roefs \& Jansen, 2004; Shide \& Rolls, 1995). In these studies, it was found that an expected high caloric content reduced intake, whereas an expected low caloric content increased intake. In these previous studies the manipulation was directed to food specific expectations on caloric content. This was different from our study in which a more general mindset was induced. We aimed to induce a more general state of mind of "letting yourself go" in the loss of control mindset versus "being in control" in the control mindset. This finding fits with cognitive models of overeating and binge eating, demonstrating that beliefs are determinants of behavior (see e.g., Beck, 2012), The finding that such a subtle mindset induction can influence intake, may provide interesting leads to design methods for reducing high caloric food intake. For example, if people during the day are induced with the belief that they are in control (e.g. by seeing short movies on their phone), this might reduce unhealthy snacking behavior.

\section{Subjective food experiences}

With regard to the influence of mindset on subjective food experiences, mixed results were found in chapters 3 and $\mathbf{4}$. In chapter $\mathbf{3}$, mindset influenced chocolate craving in the expected direction. That is, people craved more chocolate after the loss of control mindset as compared to the control mindset. We did not find significant effects of these mindsets on subjective feelings of control or hunger levels. So, our mindset induction only influenced subjective food cravings and not feelings of control or hunger. The finding that subjective craving is influenced by mindset is in line with previous research. Two recent studies likewise showed that food cravings and food valuation were affected by cognitive strategies, like regulation or reappraisal of food cravings (Boswell et al., 2018; Reader et al., 2018). The by-mindset-increased food cravings were associated with an increase in intake, which is in line with earlier studies showing that food cue reactivity and craving systematically and prospectively predict eating (Boswell \& Kober, 2016).

Note that labels indicating alleged caloric content (low, medium, high) did not significantly influence subjective experience ratings (chapter 4). In this study, subjective ratings of liking, hunger, fullness and thirst were measured on three separate days after drinking an isocaloric-sweetened beverage presented with a 
low- mid- or high-caloric caloric content label. The subjective experiences did not differ significantly between the three differently labeled drinks. This finding is not in line with previous studies, which did show that a caloric content label influenced subjective experiences (e.g., Crum, Corbin, Brownell, \& Salovey, 2011). Possibly, the manipulation of the perceived caloric content in our study was not elaborate enough, as we only provided participants with the caloric label and the general experiment information that we were interested in the effect of a sweetened low-, mid- or highcaloric beverage on metabolic responses. For example, Crum and colleagues (2011), presented the experimental drinks not only with caloric information, but included tailor-made designed labels, describing one of the drinks as indulgent and the other as sensible. Participants were asked to observe and read the label carefully before drinking the milkshakes. In hindsight, our manipulation, as compared to the study of Crum et al., (2011), therefore may not have been extensive enough and factors that emphasize a real-life product or trigger craving should be taken into account.

In general, the findings on subjective food experiences in this dissertation indicate that a loss of control mindset significantly increased the level of cravings, but not the feelings of control or hunger, as compared to a control mindset. In addition, it was shown that a mindset induced by different caloric content labels of a sweetened beverage did not affect subjective ratings significantly. So, more than just information seems to be needed to affect the subjective experience.

\section{Neural responses to visual food stimuli}

\section{Univariate analyses}

In chapters 2 and 3, the effect of mindset on neural responses to food was investigated. For the level of neural activity (mass-univariate analyses), we expected increased activity in the mesocorticolimbic system to food in the loss of control / hedonic mindset, whereas, more control-related activity was expected to food in the control / neutral mindset. Crucial differences between the studies of chapter $\mathbf{2}$ and $\mathbf{3}$ are the type of mindsets and the way mindsets were induced. In chapter $\mathbf{2}$, mindset was induced by the task performed by the participants while in the scanner. That is, to perform the task correctly, participants were constantly required to either focus on the taste (hedonic attentional focus) or on the colors (neutral attentional focus) of the foods presented in the pictures. In chapter 3, mindset was induced by brief movie clips inducing either a control or loss of control mindset, which were presented prior 
to scanning. During scanning participants attentively viewed chocolate or neutral pictures and, at regular intervals, movie-stills were presented during scanning to maintain the induced mindset.

In the paradigm used in chapter 2, females with overweight were required to apply one of two attentional foci during a fast-paced 1-back task: a hedonic focus or a neutral focus while being presented with palatable and unpalatable high-caloric food stimuli. The palatable and unpalatable stimuli were individually tailored for each participant, resulting in a set of strongly liked and strongly disliked foods for each participant. By using this 1-back task, we overcome the disadvantages of often employed passive viewing paradigms, in which the researcher cannot be certain of the mental processes during scanning (Martin et al., 2010; Rothemund et al., 2007; Stoeckel et al., 2008; Thomas et al., 2015), and thereby avoid the trap of reverse inference. Reverse inference entails inferring the likelihood of a particular mental process (i.e., reward responsiveness) from a pattern of brain activity (i.e., activity in the mesocorticolimbic system). (Poldrack, 2011). The problem with reverse inference is that brain areas could be associated with several mental processes. fMRI is designed to elucidate neural correlates of a known mental process, that is, linking activity in a brain area to cognitive function. Therefore, to be able to adequately interpret an observed pattern of neural activity, it is necessary to be certain about the ongoing mental process.

The two main findings resulting from the mass-univariate analyses of the study of chapter 2 include: (1) The level of neural activity was not significantly different between palatable and unpalatable food stimuli. (2) Independent of food palatability, several brain regions of the mesocorticolimbic system (e.g., medial orbitofrontal cortex; OFC) responded more strongly with a hedonic focus than with a neutral focus, while exactly the same visual food stimuli were presented. This finding is not in line with popular theorizing in the field, as neural activity in the mesocorticolimbic system in response to food is often interpreted as meaning that the food has a high reward value (e.g. see review of Volkow, Wang, \& Baler, 2011). Note that this line of reasoning entails reverse inference, deducing mental function from neural activity. In many of these previous studies (Martin et al., 2010; Rothemund et al., 2007; Stoeckel et al., 2008; Thomas et al., 2015), the mental process was not clear-cut, as the employed task was often simply passive viewing of food pictures.

Importantly, many previous studies did not individually tailor food stimuli, and did not include unpalatable food stimuli (Martin et al., 2010; Pursey et al., 2014; 
Rothemund et al., 2007; Stoeckel et al., 2008; Thomas et al., 2015; Yokum et al., 2011). As the highly palatable and highly unpalatable food stimuli were likely similarly salient, but differed in reward value, and because a hedonic focus may highlight that saliency, our findings suggest that neural responses to visual food stimuli may reflect motivational saliency instead of reward value. Note that previous studies - by omitting unpalatable food stimuli - were not able to distinguish between reward value and saliency.

Note that our findings were in line with other studies, which similarly showed that activity in the mesocorticolimbic system (e.g., in the medial OFC) was associated with both positive and negative value (Chikazoe et al., 2014; Kahnt et al., 2014; Rothkirch et al., 2012; Suzuki et al., 2017). For example, it was observed that neural activity in the OFC was not significantly different in response to rewarding versus aversive stimuli (Chikazoe et al., 2014; Kahnt et al., 2014; Suzuki et al., 2017). Also, the medial OFC was found to be specifically involved in reflecting automatic processing of implicit motivational salience (Rothkirch et al., 2012). So, taken together, it seems fair to conclude that the level of neural activity in the mesocorticolimbic system reflects saliency independent of valence.

In chapter 3, a loss of control and a control mindset were induced by presenting normal-weight female chocolate lovers with a short movie. Note that all participants viewed both movies in two separate sessions (i.e., a within-subjects design). Neural responses to visual chocolate stimuli as compared to neutral stimuli (office supplies) were measured using fMRI with an ultra-high field scanner (7T). Unexpectedly, neural responding to chocolate versus neutral stimuli was not significantly influenced by the induced mindset of loss of control versus control. As expected, in response to palatable food stimuli, neural activity in several regions of the mesocorticolimbic system was significantly stronger for chocolate than for neutral stimuli.

A possible explanation for not finding the hypothesized effect of the induced mindsets, is that all participants scored quite high on trait chocolate craving. It might be that this trait chocolate craving dominated by automatic neural responses over the quite subtly induced loss of control versus control mindset (Giuliani et al., 2018; Miedl et al., 2018). There is indeed some evidence (Miedl et al., 2018) that high trait chocolate cravers show more implicit pleasure-related neural responses in the mesocorticolimbic system towards chocolate pictures as compared to low chocolate cravers, without strict instructions during viewing. In addition, the mindset induction was not task-based and was quite short. Though movie-stills were repeatedly 
presented during task-performance, it could very well be that this induced mindset disappeared into the background during task performance.

Taken together, from the fMRI studies in this dissertation it can be concluded that an induced mindset can influence the level of neural activity, but this seems to be only true when the mindset manipulation is continuously active during the measurement of neural activity. Actively engaging with a mindset to the presented stimuli might be important for two reasons: 1) As it is known that high caloric foods have a double-sided nature (i.e., high hedonic value and simultaneously a low health value), it cannot be assumed that the hedonic value automatically is processed first, and participants may need constant reminding (Roefs et al., 2018), and 2) With a task-based manipulation, the researcher can be relatively certain about the ongoing mental process while the participant is in the scanner. Therefore, it limits the risks of reverse inference and contributes to further elucidating the ambiguous function of the mesocorticolimbic system (Poldrack, 2011).

\section{Multivariate analyses}

Whereas univariate analyses of fMRI data are informative regarding involvement of brain areas in certain tasks, multivariate analysis can decode representational content in the brain (Norman et al., 2006). So, visual food stimuli (e.g., highly palatable food and highly unpalatable food) could lead to a similar level of activation, whereas the multivoxel pattern of activation to those stimuli could differ (see Figure 2 on page 14). These two (i.e. univariate and multivariate) analysis approaches could therefore lead to different insights.

Interestingly, in chapter 2 it was shown that palatable and unpalatable food stimuli could be distinguished above chance from multivoxel patterns of neural activity, and this distinction was mostly successful for data acquired when the participant's task required a hedonic attentional focus. Observed brain regions with above-chance classification included: regions of the mesocorticolimbic system (right putamen, bilateral insula, caudate, right anterior cingulate and bilateral dorsolateral and medial prefrontal cortices). So, whereas the level of neural activity was not significantly different between palatable and unpalatable foods, multivoxel patterns of neural activity were significantly different between these two types of foods. The level of neural activity to palatable and unpalatable food stimuli might reflects saliency, whereas the neural pattern might reflect valence (positive or negative). This suggested interpretation is in line with prior research that showed that the subjective 
value of food stimuli could be specifically decoded from multi-voxel patterns of neural activity, whereas the level of neural activity was not significantly different for positive versus negative stimuli (Chikazoe et al., 2014; Suzuki et al., 2017).

In chapter 3, the multivariate analysis did not lead to different insights than the univariate analysis. Chocolate versus neutral stimuli could be decoded above chance from multivoxel patterns of neutral activity, but decoding accuracy was not significantly different between the two induced mindsets (control or loss of control). So, as with the univariate analyses, neural responding was different for chocolate versus neutral stimuli, but was not significantly affected by mindset. The same explanations for a lack of significant effect of mindset apply here. That is, the mindset induction was quite subtle and not task-based (see univariate analyses).

From the multivariate analyses, it can be concluded that valence (i.e. food palatability) of food stimuli can be decoded from multivariate patterns, mostly when data were acquired with a hedonic focus. Multivariate neural patterns were not significantly different while processing chocolate stimuli in a control versus a lossof-control mindset. So, these results again point to the benefits of using a strong task-based manipulation, guaranteeing increased control over the engaged mental process.

\section{Effect of food cue exposure on neural responses to food stimuli}

In chapter 5, the effect of a food cue exposure therapy as compared to a lifestyle control intervention was investigated on neural responses to high-caloric food stimuli. Forty-five women with overweight were randomly assigned to either a cue-exposure intervention ( $n=23$ ) or a control lifestyle intervention ( $n=22$ ) (van den Akker et al., 2016). Of this sample 10 women ( $n=5$, cue-exposure intervention and $n=5$, control lifestyle intervention) participated in the fMRI study. Before and after treatment, neural responses to individually tailored palatable high-caloric food stimuli were measured using $\mathrm{fMRI}$, in the same paradigm as was used in the research described in chapter 2. Because of the small sample size, data were analyzed case-by-case. We expected that cue exposure therapy, as compared to a lifestyle control intervention, would lead to reduced neural activity in food-cue-reactivity-related brain regions and increased neural activity in inhibitory-control brain regions when viewing high-caloric food stimuli. The hedonic focus condition was expected to lead to more pronounced results as this focus is aligned with the experience of craving. 
However, contrary to our hypothesis, cue exposure therapy - as compared to the lifestyle control intervention - did not lead to a significantly stronger reduction from pre to post treatment in neural responding to visual high-caloric palatable foods in food-cue-reactivity-related brain regions. Instead, most participants showed a decrease in neural activity in food-cue-reactivity related brain regions after receiving the lifestyle intervention. The decreased food-cue-reactivity related brain activity for these participants was more pronounced and widespread after receiving the lifestyle intervention as compared to participants that had received the food cue exposure therapy. In addition, these findings were stronger in the hedonic attentional focus condition. When assessing activity in brain regions related to inhibitory control, also no significant differences were found between participants that received food cue exposure therapy or a lifestyle intervention.

An explanation for these unexpected results may be found in the proposed mechanism by which cue exposure is thought to work: inhibitory learning (Bouton, 2004, 2011; Jansen et al., 2016). Food cue exposure therapy initiates inhibitory learning with a focus on the principle of extinction (learning a new association) and that is, importantly, context-dependent and food-specific (Bouton, 2004, 2011; Jansen et al., 2016). More specifically, during food cue exposure therapy, participants learn a new inhibitory association (the cue does not predict intake), which then exists next to the original disinhibiting association (the cue does predicts intake) (Jansen et al., 2016). That means that the food-cue-intake association is not erased, and therefore the food cues in the fMRI task might still trigger neural food cue reactivity. Moreover, both the context (fMRI scanner versus a laboratory room, participants' home and other relevant contexts) and the food stimuli differed between our fMRI measurement and the therapy setting. As extinction of food cue reactivity is learned in a different context than during our measurement, this could explain why we did not observe any significant reduction in neural food cue reactivity.

Furthermore, cue exposure intervention typically leads to reduced consumption of only the exposed foods, while not generalizing to a reduction in consumption of other foods (Schyns et al., 2016, 2018, 2019). The food cues used during cue exposure therapy and the fMRI task were not similar. Therefore, is it unlikely that the food cues included in the fMRI task would trigger a similar decreased food cue reactivity as provoked during the cue exposure therapy. In an earlier study of our lab (Frankort et al., 2014), a reduction in neural food cue reactivity in response to food cues was found after cue exposure. Importantly, the cue exposure and measurement of neural 
activity both took place in the scanner while using the same food stimuli throughout (i.e., chocolate stimuli). So, food cues and context should be similar for optimally examining the effects of cue exposure on neural responding to food cues.

Importantly, during the life style intervention, participants received education on dieting and healthy weight loss and on nutrients and energy balance (van den Akker et al., 2016). In contrast to the cue exposure therapy, focus was not on specific favourite foods. Instead, negative health consequences of high caloric foods in general was highlighted, which may have contributed to participants' reduced neural responses to high-caloric foods. This interpretation aligns well with previous studies, showing that focusing on negative health aspects can reduce neural activity to visually presented high-caloric food stimuli (Hollmann et al., 2012; Siep et al., 2012). Note that due to the small sample size, these findings should be interpreted with caution. Larger-scale studies should first replicate and/or extend these findings.

\section{Hormones}

In chapter 3, the influence of a loss of control versus a control mindset on the "hunger hormone" ghrelin (Kojima et al., 1999) and the "satiety hormone" GLP-1 (Drucker, 2006) before and after eating chocolate was measured. It was expected that a loss of control mindset would result in an increased ghrelin response reflecting increased hunger, even after eating, and a decreased GLP-1 response reflecting decrease in satiety as compared to the manipulated control mindset. However, the findings on hormonal responses in chapter 3 did not confirm our expectations. No significant differences in ghrelin and GLP-1 responses were found between the two induced mindsets. Hormones did respond as expected over time and in response to eating, where ghrelin levels decreased, and GLP-1 levels increased after eating moments.

The current findings are not in line with previous studies on this topic, which did find that specific expectations or beliefs influenced hormonal responding to food (i.e., levels of ghrelin and GLP-1); Cassady, Considine, \& Mattes, 2012; Crum et al., 2011). A potentially important difference between our study and these earlier studies is that Cassady et al (2012) and Crum et al (2011) induced expectations focused on the effects of ingestion. In our study, we induced a more general mindset, and we did not induce any expectations about the satiating effects or caloric load of the eaten chocolate. 


\section{Metabolism}

In chapter 4, we assessed whether expected caloric content as indicated on productlabels influenced the metabolic responses to drinking equally sweet and isocaloric beverages. The metabolic response was measured with indirect calorimetry. The resting energy expenditure (REE) before and after consumption of the labeled beverages were measured to calculate the thermic effect of food (TEF). The TEF is related to the energy required to process and metabolize the consumed food or drink (Reed \& Hill, 1996). It was expected that consuming a beverage with a highcaloric label would lead to a higher TEF as compared to a beverage with a low-caloric label.

Unexpectedly, we found that, while consuming the beverages did elicit a metabolic response pre-to post consumption, these effects were not significantly different between the three label conditions. So, expected caloric content did not significantly influence the TEF of the beverages. Previous studies have shown that metabolism is influenced by actual caloric content (Crovetti et al., 1998; Quatela et al., 2016) and by sweetness (Imamura et al., 2015; Just et al., 2008). However, to our knowledge this was the first attempt to test the influence of anticipated caloric content on the TEF.

Therefore, our findings suggest that metabolism responds in a purely physiological way to the actual calories consumed and is not significantly affected by anticipated caloric content. An explanation for the lack of effect could be that the anticipation induced by the caloric content labels was not strong enough. Note that previous studies have found that labels providing caloric information influence hormonal responses (i.e. ghrelin and GLP-1 levels) (Cassady et al., 2012; Crum et al., 2011). So, a stronger manipulation of anticipated caloric content might affect metabolism.

\section{The trap of reverse inference}

Results from previous studies on neural responding to food stimuli are highly inconsistent (Ziauddeen et al., 2012), and this dissertation suggests that the lack of well-controlled fMRI paradigms may have contributed to this inconsistency. That is, in most previous studies, the exact ongoing mental process while people were in the scanner was unclear. Were they thinking of the taste, the calories, the price, or even other aspects of the presented food stimuli? 
To carry the field forward, it is important to be sure of the exact ongoing mental process of participants while they perform the task in the scanner. It is important to account for the double-sided nature of high caloric foods. That is, high caloric foods have a high hedonic value and simultaneously a low health value, and it cannot be assumed that hedonic value always takes precedence when people are presented with food stimuli (Roefs et al., 2018). However, this is exactly the assumption in much previous research. That is, it has been assumed that neural responses to high caloric food stimuli reflect a hedonic evaluation of the food stimuli (Martin et al., 2010; Rothemund et al., 2007; Stoeckel et al., 2008; Thomas et al., 2015). A conclusion like this is a form of reverse inference: deducing mental function from observed neural activity. The probability of reverse inference is high if activity in a certain brain area is related to many different mental processes, and/or if the precise ongoing mental process engaged during the task is unclear (Poldrack, 2011). Given as an example of Poldrack (2011), and based on an online database (http://www.neurosynth.org), is the anterior insula which is a brain area involved in many different mental processes. Activity in the anterior insula combined with an uncertainty about the mental process engaged during the fMRI task makes it hard to interpret the precise role activity in the anterior insula. Note that the precise mental process is unknown in previous passive viewing paradigms presenting visual food stimuli, because the researcher is unaware of how the participants process the presented food stimuli, and the mental process may fluctuate over the course of the scanning. So, with fMRI it is possible to learn more about neural activity, but only if the ongoing mental process is clear.

A danger of reverse inference obviously is that the wrong mental process is inferred based on the observed neural activity. As our research clearly showed that the level of neural activity does not differ significantly between highly palatable versus highly unpalatable foods, one cannot interpret this level of neural activity as reflecting reward value. The level of neural activity in the mesocorticolimbic system is not proportionate to the reward value of the foods. Instead, activity in this system may reflect motivational saliency, be it negative or positive (Salamone \& Correa, 2012). Value may instead be inferred from multivoxel patterns of neural activity (Chikazoe et al., 2014; Norman et al., 2006; Suzuki et al., 2017). As a last note, the trap of reverse inference definitely is not only relevant for the field of neural food processing as discussed here, but also should be taken into consideration while designing fMRI tasks to assess neural activity involved in cognitive processes in general. 


\section{Conclusion}

The main findings are displayed in Figure 1, and the main conclusions and brief interpretations of this dissertation are described below

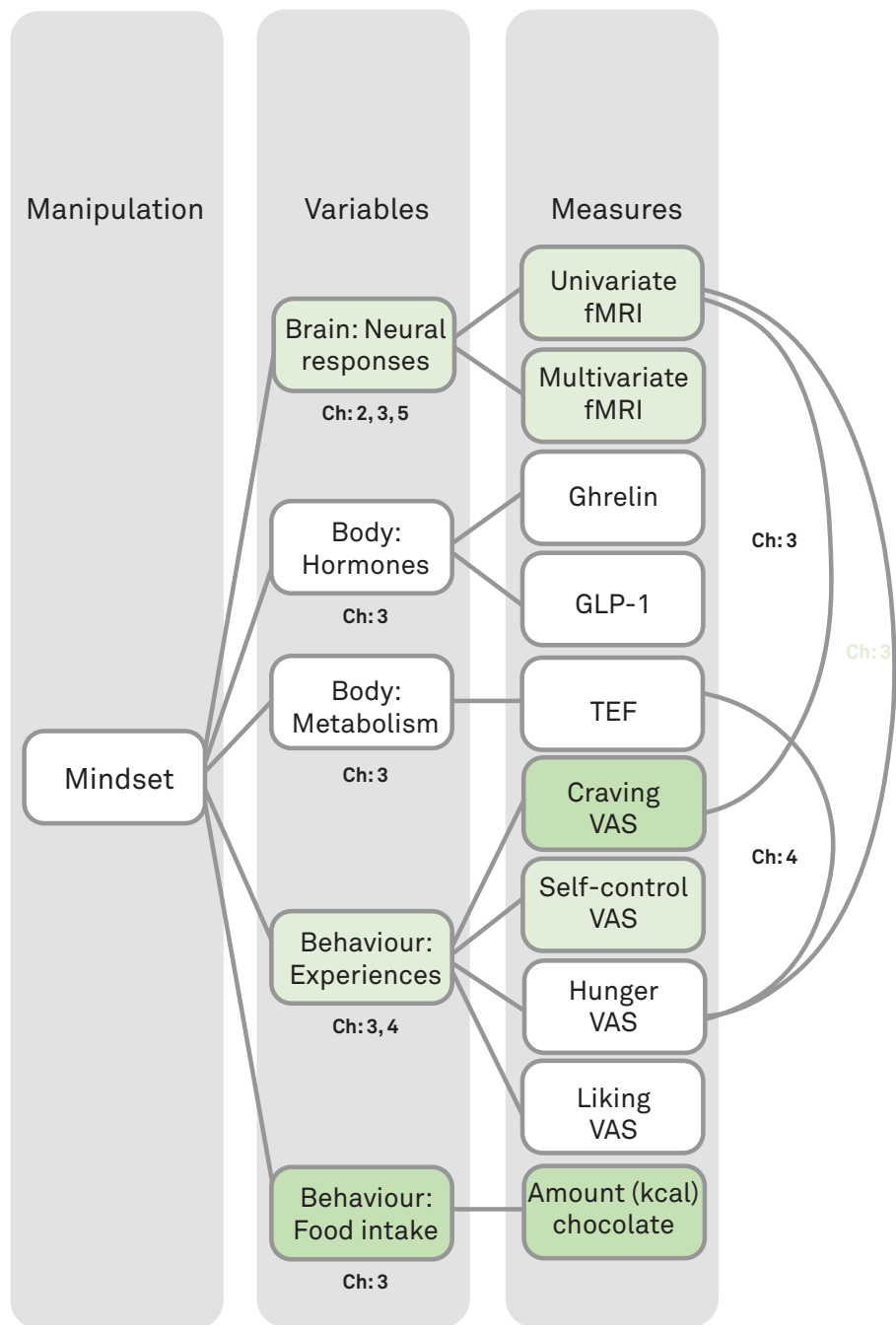

Figure 1. Manipulation, variables and measures and their relationships assessed in this dissertation. Abbreviations: Ch = chapter, fMRI = functional Magnetic Resonance Imaging, GLP-1 = Glucagon-like Peptide 1, TEF = Thermic Effect of Food, VAS = visual analogue scale. Green boxes represent variables and measures for which a significant effect of mindset was observed, with darker green representing more consistent evidence. White boxes and grey arrows represent non-significant findings. 


\section{Main conclusions and brief interpretations}

1. Attentional focus has a strong effect on the level of neural activity elicited by high-caloric visual food stimuli in females with overweight. Where mass-univariate analyses could hardly distinguish between palatable and unpalatable food stimuli, remarkable differences were found between a hedonic focus versus a neutral focus when collapsing over food palatability. That is, several relevant brain regions (e.g., medial OFC) responded more strongly in a hedonic compared to neutral focus when processing exactly the same visual stimuli. As the level of neural activity was not proportionate to the palatability of the presented foods, these findings cast doubt on the general and popular view that the level of neural activity elicited by high caloric food stimuli reflects the reward-value of these food stimuli.

2. Multivoxel patterns of neural activity were significantly different for palatable versus unpalatable food stimuli, mainly for the hedonic attentional focus. As the level of neural activity was not significantly different for palatable versus unpalatable foods, this illustrates the clear difference between examining the involvement of brain areas and the representational content of brain areas, as reflected in multivoxel patterns (Norman et al., 2006).

3. A more subtly induced mindset (control versus loss of control) influenced food intake and craving for chocolate. That is, healthy-weight females ate and craved more chocolate when in a loss of control mindset as compared to a control mindset. These findings illustrate that an loss of control mindset can influence craving experiences and intake in eatingrelated contexts.

4. The induction of a loss of control mindset or control mindset did not lead to differences in neural responses to visual chocolate stimuli or hormonal responses (i.e. ghrelin and GLP-1 levels) to food. One explanation for not finding the neural effects is that is that all participants included scored high on trait chocolate craving which could lead to automatically strong neural response to chocolate stimuli (but see 3 : subjective and behavioral differences were found between the control manipulations). It could also be that the mindset induction was not explicit enough to influence neural responses as it was not part of fMRI task requirements. Contrary 
to previous research examining mindset effects on hormonal responses, is that no expectations about the satiating effects or caloric load of the chocolate were induced. This might suggest it is essential to take into account trait characteristics, the nature of the fMRI task, and food-related expectations. It could also be a control/loss of control mindset are only relevant for actual eating behaviour, and do not influence physiological neuronal and hormonal responses.

5. A remarkable observation that can be derived from points 3 and 4, is that physiological (neural or hormonal) responding, behaviour (intake) and subjective experiences (craving) as elicited by mindset are incongruent. So, physiological responses, subjective experiences and food intake do not go hand in hand.

6. Metabolism, as measured with the TEF in healthy-weight participants, was found to be irresponsive to anticipated caloric content as indicated by labels, and only responded to actual caloric intake. An explanation for the lack of significant effect could be that the intake anticipation indicated by the caloric content labels was not strong enough, or that the caloric load as suggested by the sweet taste overruled the anticipated caloric load as indicated by the labels.

7. Cue exposure therapy did not lead to a stronger reduction in neural activity in response to high caloric food stimuli in food-cue-reactivity-related brain regions. This lack of effect in participants receiving cue-exposure therapy might be due to the fact that cue-exposure therapy does not erase previously learned cue-intake relationships. Instead, due to this therapy, inhibitory relationships (cue - no intake) are learned, which then exist alongside the original cue-intake relationships. Therefore, the food stimuli in the fMRI task might still trigger neural food cue reactivity. Note that these results should be interpreted with caution as only 10 participants were included of the overall intervention group $(n=45)$.

8. Instead, reduced neural food cue reactivity was more pronounced and more widespread, and mostly with a hedonic attentional focus, in participants that received a lifestyle intervention. This can likely be explained by the raised awareness of the negative health consequences 
of high caloric foods in general during the lifestyle intervention. Although participants were instructed during scanning to attend to the hedonic aspects of the foods presented, this lifestyle training may have interfered with this hedonic focus during the post-intervention scanning-session by increasing awareness of negative health aspects.

\section{Implications and future directions}

Taken together, the research in this dissertation shows that mindset affects neural responding to food stimuli, but only when the manipulation is strong, and task based. Mindset also influences actual eating behavior, but it does not affect specific postingestive metabolism and hormonal responding and the accompanying subjective experiences. In addition, a food cue exposure intervention did not lead to significant reduced effects on neural activity as elicited by visual food stimuli.

An important factor to take into account with a mindset manipulation is the believability and target of the mindset. For example, when a mindset is relevant for behavior in an eating relevant context, it results in a bigger effect than in a more general setting. More specifically, in chapter 3 it was shown that a loss of control mindset led to more chocolate intake. Importantly, on that moment, the mindset was relevant as actual eating occurred. During the fMRI paradigm in the same study, with just viewing chocolate stimuli, no effect of mindset was observed. In addition, if the mindset manipulation was explicit and task-based (see chapter 2), attentional focus strongly affected neural responding. So, our findings are in line with previous research that found that a mindset is effective in changing food-related cognition (e.g. see Frankort et al., 2012; Siep et al., 2012; Werthmann, Jansen, \& Roefs, 2016), however, only on a relevant moment and in a relevant context.

Current lifestyle interventions for overweight are not effective in the long run as the majority of overweight individuals regain lost weight within five years (for a review see Franz et al., 2007). It is conceivable that a lifestyle intervention will trigger a temporary health mindset, which could help to lose weight. However, this health mindset may not stick after therapy has ended, contributing to relapse. It might be beneficial for treatment to more frequently and for a longer duration induce this health mindset. In addition, it might be beneficial to induce this health mindset specifically on 'high-risk' moments. One way of intervening for a longer 
duration in people's daily life is by using an Ecological Momentary Intervention (EMI), administered via smartphones. When combined with predictive algorithms, EMI also permits intervening on specifically 'at risk' moments, that is, when a person is about to consume high caloric foods (Boh et al., 2016; Heron \& Smyth, 2010). This might improve the effectiveness of a health mindset induction, as treatment can be delivered on the right moment and in the right context, personalized for each individual.

Another future direction is to examine the influence of mindset on neural responses during actual eating. This could be achieved by using an fMRI setup using a so-called gustometer to deliver liquid tastes. Previous research suggests differences between the anticipatory (e.g. seeing visual food stimuli) as compared to the consummatory (e.g. actually tasting/eating food stimuli) neural (rewarding) responding to foods in overweight people (D'Agostino \& Small, 2012; Rothemund et al., 2007; Eric Stice et al., 2008). However, up until now, there are limited studies investigating the cognitive influences on neural correlates of consumption (D'Agostino \& Small, 2012). It could therefore be valuable to examine this relationship more in future research. In addition, as it seems to be of importance to successful interfere with a mindset on the relevant moment (as indicated by this dissertation), examining neural responses while inducing a mindset during actual food intake would be valuable. 
REFERENCES 
Abdallah, L., Chabert, M., \& Louis-Sylvestre, J. (1997). Cephalic phase responses to sweettaste. The American Journal of Clinical Nutrition, 65(3), 737-743. https://doi.org/10.1093/ ajcn/65.3.737

Afshin, A., Forouzanfar, M. H., Reitsma, M. B., Sur, P., Estep, K., Lee, A., Marczak, L., Mokdad, A. H., Moradi-Lakeh, M., Naghavi, M., Salama, J. S., Vos, T., Abate, K. H., Abbafati, C., Ahmed, M. B., Al-Aly, Z., Alkerwi, A., Al-Raddadi, R., Amare, A. T., ... Murray, C. J. L. (2017). Health Effects of Overweight and Obesity in 195 Countries over 25 Years. New England Jour nal of Medicine, 377(1), 13-27. https://doi.org/10.1056/NEJMoa1614362

American Psychiatric Association. (2013). Diagnostic and statistical manual of mental disorders (5th ed.). American Psychiatric Publishing.

Appelhans, B. M. (2009). Neurobehavioral Inhibition of Reward-driven Feeding: Implications for Dieting and Obesity. Obesity, 17(4), 640-647. https://doi.org/10.1038/oby.2008.638

Bartoshuk, L ., Duffy, V ., Green, B. ., Hoffman, H. ., Ko, C.-W., Lucchina, L. , Marks, L. , Snyder,D., \& Weiffenbach, J.. (2004). Valid across-group comparisons with labeled scales: the gLMS versus magnitude matching. Physiology \& Behavior, 82(1), 109-114. https:// doi.org/10.1016/j.physbeh.2004.02.033

Baumeister, H., \& Härter, M. (2007). Mental disorders in patients with obesity in comparison with healthy probands. International Journal of Obesity, 31(7), 1155-1164. https://doi. org/10.1038/sj.ijo.0803556

Baynes, K. C., Dhillo, W. S., \& Bloom, S. R. (2006). Regulation of food intake by gastrointestinal hormones. Current Opinion in Gastroenterology, 22(6), 626-631. https://doi org/10. 1097/01.mog.0000245537.43142.63

Beck, J. S. (2012). The Beck Diet Solution: Train your brain to think like a thin person. Hachette UK.

Bell, C. G., Walley, A. J., \& Froguel, P. (2005). The genetics of human obesity. Nature Reviews Genetics, 6(3), 221-234. https://doi.org/10.1038/nrg1556

Benson, K., \& Raynor, H. A. (2014). Occurrence of habituation during repeated food exposure via the olfactory and gustatory systems. Eating Behaviors, 15(2), 331-333. https://doi. org/10.1016/j.eatbeh.2014.01.007

Berridge, K. C., Ho, C. Y., Richard, J. M., \& Difeliceantonio, A. G. (2010). The tempted brain eats: Pleasure and desire circuits in obesity and eating disorders. Brain Research, 1350, 43-64. https://doi.org/10.1016/j.brainres.2010.04.003

Berthoud, H.-R., Lenard, N. R., \& Shin, A. C. (2011). Food reward, hyperphagia, and obesity. American Journal of Physiology-Regulatory, Integrative and Comparative Physiology, 300(6), R1266-R1277. https://doi.org/10.1152/ajpregu.00028.2011

Bhanji, J. P., \& Beer, J. S. (2012). Taking a different perspective: Mindset influences neural regionsthat represent value and choice. Social Cognitive and Affective Neuroscience, 7(7), 782-793. https://doi.org/10.1093/scan/nsr062 
Bish, C. L., Blanck, H. M., Serdula, M. K., Marcus, M., Kohl, H. W., \& Khan, L. K. (2005). Diet and Physical Activity Behaviors among Americans Trying to Lose Weight: 2000 Behavior al Risk Factor Surveillance System. Obesity Research, 13(3), 596-607. https://doi. org/10.1038/oby.2005.64

Boh, B., Jansen, A., Clijsters, I., Nederkoorn, C., Lemmens, L. H. J. M., Spanakis, G., \& Roefs, A. (2016). Indulgent thinking? Ecological momentary assessment of overweight and healthy-weight participants' cognitions and emotions. Behaviour Research and Therapy, 87, 196-206. https://doi.org/10.1016/j.brat.2016.10.001

Boswell, R. G., \& Kober, H. (2016). Food cue reactivity and craving predict eating and weight gain: a meta-analytic review. Obesity Reviews, 17(2), 159-177. https://doi.org/10.1111/ obr.12354

Boswell, R. G., Sun, W., Suzuki, S., \& Kober, H. (2018). Training in cognitive strategies reduces eating and improves food choice. Proceedings of the National Academy of Sciences of the United States of America, 115(48), E11238-E11247. https://doi.org/10.1073/ pnas.1717092115

Boutelle, K. N., \& Bouton, M. E. (2015). Implications of learning theory for developing programs to decrease overeating. Appetite, 93, 62-74. https://doi.org/10.1016/j.appet. 2015.05.013

Bouton, M. E. (2004). Context and Behavioral Processes in Extinction. Learning \& Memory, 11(5), 485-494. https://doi.org/10.1101//m.78804

Bouton, M. E. (2011). Learning and the persistence of appetite: Extinction and the motivation to eat and overeat. Physiology \& Behavior, 103(1), 51-58. https://doi.org/10.1016/j. physbeh.2010.11.025

Brooks, S. J., ODaly, O. G., Uher, R., Friederich, H. C., Giampietro, V., Brammer, M., Williams, S. C. R., Schiöth, H. B., Treasure, J., \& Campbell, I. C. (2011). Differential neural responses to food images in women with bulimia versus anorexia nervosa. PLOS ONE, 6(7). https://doi.org/10.1371/journal.pone.0022259

Brunstrom, J. M., \& Mitchell, G. L. (2007). Flavor-nutrient learning in restrained and unrestrained eaters. Physiology \& Behavior, 90(1), 133-141. https://doi.org/10.1016/j. physbeh.2006.09.016

Burger, K. S., \& Berner, L. a. (2014). A functional neuroimaging review of obesity, appetitive hormones and ingestive behavior. Physiology \& Behavior, 136, 121-127. https://doi. org/10.1016/j.physbeh.2014.04.025

Burke, M. V., \& Small, D. M. (2015). Physiological mechanisms by which non-nutritive sweetenersmay impact body weight and metabolism. Physiology \& Behavior, 152(Pt B), 381-388. https://doi.org/10.1016/j.physbeh.2015.05.036

Cassady, B. a., Considine, R. V., \& Mattes, R. D. (2012). Beverage consumption, appetite, and energy intake: what did you expect? American Journal of Clinical Nutrition, 95(3), 587-593. https://doi.org/10.3945/ajcn.111.025437 
Chambers, E. S., Bridge, M. W., \& Jones, D. A. (2009). Carbohydrate sensing in the human mouth: effects on exercise performance and brain activity. The Journal of Physiology, 587(8), 1779-1794. https://doi.org/10.1113/jphysiol.2008.164285

Chaudhri, O. B., Wynne, K., \& Bloom, S. R. (2008). Can gut hormones control appetite and prevent obesity? Diabetes Care, 31 Supp/ 2, S284-9. https://doi.org/10.2337/dc08-s269

Chikazoe, J., Lee, D. H., Kriegeskorte, N., \& Anderson, A. K. (2014). Population coding of affect across stimuli, modalities and individuals. Nature Neuroscience, 17(8), 1114-1122. https://doi.org/10.1038/nn.3749

Clithero, J. A., \& Rangel, A. (2014). Informatic parcellation of the network involved in the computation of subjective value. Social Cognitive and Affective Neuroscience, 9(9), 1289-1302. https://doi.org/10.1093/scan/nst106

Cox, D. D., \& Savoy, R. L. (2003). Functional magnetic resonance imaging (fMRI) "brain reading": detecting and classifying distributed patterns of fMRI activity in human visual cortex. Neurolmage, 19(2), 261-270. https://doi.org/10.1016/S1053-8119(03)00049-1

Cox, R. W. (1996). AFNI: Software for Analysis and Visualization of Functional Magnetic Resonance Neuroimages. Computers and Biomedical Research, 29(3), 162-173. https:// doi.org/10.1006/cbmr.1996.0014

Craske, M. G., Treanor, M., Conway, C. C., Zbozinek, T., \& Vervliet, B. (2014). Maximizing exposure therapy: An inhibitory learning approach. Behaviour Research and Therapy, 58, 10-23. https://doi.org/10.1016/j.brat.2014.04.006

Cremers, H. R., Wager, T. D., \& Yarkoni, T. (2017). The relation between statistical power and inference in fMRI. PloS One, 12(11), e0184923. https://doi.org/10.1371/journal. pone.0184923

Crovetti, R., Porrini, M., Santangelo, A., \& Testolin, G. (1998). The influence of thermic effect of food on satiety. European Journal of Clinical Nutrition, 52(7), 482-488. https://doi.org/ 10.1038/sj.ejcn.1600578

Crum, A. J., Corbin, W. R., Brownell, K. D., \& Salovey, P. (2011). Mind over milkshakes: Mindsets, not just nutrients, determine ghrelin response. Health Psychology, 30(4), 424-429. https://doi.org/10.1037/a0023467

D’Agostino, A. E., \& Small, D. M. (2012). Neuroimaging the interaction of mind and metabolism in humans. Molecular Metabolism, 1(1-2), 10-20. https://doi.org/10.1016/j.molmet. 2012.06.002

Dailey, M. J., \& Moran, T. H. (2013). Glucagon-like peptide 1 and appetite. Trends in Endocrinology and Metabolism: TEM, 24(2), 85-91. https://doi.org/10.1016/j.tem. 2012.11.008

Dalenberg, J. R., Patel, B. P., Denis, R., Veldhuizen, M. G., Nakamura, Y., Vinke, P. C., Luquet, S., \& Small, D. M. (2020). Short-Term Consumption of Sucralose with, but Not without, Carbohydrate Impairs Neural and Metabolic Sensitivity to Sugar in Humans. Cell Metabolism, 31(3), 493-502.e7. https://doi.org/10.1016/j.cmet.2020.01.014 
Davidson, T. L., \& Swithers, S. E. (2004). A Pavlovian approach to the problem of obesity. International Journal of Obesity, 28(7), 933-935. https://doi.org/10.1038/sj.ijo.0802660

Desmond, J. E., \& Glover, G. H. (2002). Estimating sample size in functional MRI (fMRI) neuroimaging studies: Statistical power analyses. Journal of Neuroscience Methods, $118,115-128$

Dreher, J.-C., Schmidt, P. J., Kohn, P., Furman, D., Rubinow, D., \& Berman, K. F. (2007). Menstrual cycle phase modulates reward-related neural function in women. Proceedings of the National Academy of Sciences, 104(7), 2465-2470. https://doi.org/10.1073/ pnas.0605569104

Drucker, D. J. (2006). The biology of incretin hormones. Cell Metabolism, 3(3), 153-165. https:// doi.org/10.1016/j.cmet.2006.01.004

Eklund, A., Nichols, T. E., \& Knutsson, H. (2016). Cluster failure: Why fMRI inferences for spatial extent have inflated false-positive rates. Proceedings of the National Academy of Sciences, 113(28), 7900-7905. https://doi.org/10.1073/pnas.1602413113

Faul, F., Erdfelder, E., Buchner, A., \& Lang, A.-G. (2009). Statistical power analyses using G*Power 3.1: tests for correlation and regression analyses. Behavior Research Methods, 41(4), 1149-1160. https://doi.org/10.3758/BRM.41.4.1149

Faulkner, G. P., Pourshahidi, L. K., Wallace, J. M. W., Kerr, M. A., McCaffrey, T. A., \& Livingstone, M. B. E. (2014). Perceived 'healthiness' of foods can influence consumers' estimations of energy density and appropriate portion size. International Journal of Obesity, 38(1), 106-112. https://doi.org/10.1038/ijo.2013.69

Feinberg, D. A., Moeller, S., Smith, S. M., Auerbach, E., Ramanna, S., Glasser, M. F., Miller, K. L., Ugurbil, K., \& Yacoub, E. (2010). Multiplexed Echo Planar Imaging for Sub-Second Whole Brain FMRI and Fast Diffusion Imaging. PLOS ONE, 5(12), e15710. https://doi. org/10.1371/journal.pone.0015710

Ferriday, D., \& Brunstrom, J. M. (2011). 'I just can't help myself': effects of food-cue exposure in overweight and lean individuals. International Journal of Obesity, 35(1), 142-149. https://doi.org/10.1038/ijo.2010.117

Fildes, A., Charlton, J., Rudisill, C., Littlejohns, P., Prevost, A. T., \& Gulliford, M. C. (2015). Probability of an Obese Person Attaining Normal Body Weight: Cohort Study Using Electronic Health Records. American Journal of Public Health, 105(9), e54-e59. https:// doi.org/10.2105/AJPH.2015.302773

Flint, A., Raben, A., Astrup, A., \& Holst, J. J. (1998). Glucagon-like peptide 1 promotes satiety and suppresses energy intake in humans. The Journal of Clinical Investigation, 101(3), 515-520. https://doi.org/10.1172/JC1990

Frankort, A., Roefs, A., Siep, N., Roebroeck, A., Havermans, R., \& Jansen, A. (2012). Reward activity in satiated overweight women is decreased during unbiased viewing but increased when imagining taste: an event-related fMRI study. International Journal of Obesity (2005), 36(5), 627-637. https://doi.org/10.1038/ijo.2011.213 
Frankort, A., Roefs, A., Siep, N., Roebroeck, A., Havermans, R., \& Jansen, A. (2014). The craving stops before you feel it: neural correlates of chocolate craving during cue exposure with response prevention. Cerebral Cortex (New York, N.Y. : 1991), 24(6), 1589-1600. https://doi.org/10.1093/cercor/bht016

Franssen, S., Jansen, A., van den Hurk, J., Roebroeck, A., \& Roefs, A. (2020). Power of mind: Attentional focus rather than palatability dominates neural responding to visual food stimuli in females with overweight. Appetite, 148(August 2019), 104609. https:// doi.org/10.1016/j.appet.2020.104609

Franz, M. J., VanWormer, J. J., Crain, A. L., Boucher, J. L., Histon, T., Caplan, W., Bowman, J. D., \& Pronk, N. P. (2007). Weight-Loss Outcomes: A Systematic Review and Meta-Analysis of Weight-Loss Clinical Trials with a Minimum 1-Year Follow-Up. Journal of the American Dietetic Association, 107(10), 1755-1767. https://doi.org/10.1016/j.jada. 2007.07.017

Gibson, C. D., Carnell, S., Ochner, C. N., \& Geliebter, a. (2010). Neuroimaging, gut peptides and obesity: Novel studies of the neurobiology of appetite. Journal of Neuroendocrinology, 22(8), 833-845. https://doi.org/10.1111/j.1365-2826.2010.02025.x

Giuliani, N. R., Merchant, J. S., Cosme, D., \& Berkman, E. T. (2018). Neural predictors of eating behavior and dietary change. Annals of the New York Academy of Sciences, 00, 1-13. https://doi.org/10.1111/nyas.13637

Grabenhorst, F., Rolls, E. T., \& Bilderbeck, A. (2008). How Cognition Modulates Affective Responses to Taste and Flavor: Top-down Influences on the Orbitofrontal and Pregenual Cingulate Cortices. Cerebral Cortex, 18(7), 1549-1559. https://doi.org/10. 1093/cercor/bhm185

Gravel, K., Doucet, É., Peter Herman, C., Pomerleau, S., Bourlaud, A., \& Provencher, V. (2012). 'Healthy,' 'diet,' or 'hedonic'. How nutrition claims affect food-related perceptions and intake? Appetite, 59(3), 877-884. https://doi.org/10.1016/j.appet.2012.08.028

Green, B. G., Dalton, P., Cowart, B., Shaffer, G., Rankin, K., \& Higgins, J. (1996). Evaluating the 'Labeled Magnitude Scale' for Measuring Sensations of Taste and Smell. Chemical Senses, 21(3), 323-334. https://doi.org/10.1093/chemse/21.3.323

Haber, S. N., \& Knutson, B. (2010). The reward circuit: linking primate anatomy and human imaging. Neuropsychopharmacology : Official Publication of the American College of Neuropsychopharmacology, 35(1), 4-26. https://doi.org/10.1038/npp.2009.129

Hall, K. D., Heymsfield, S. B., Kemnitz, J. W., Klein, S., Schoeller, D. a., \& Speakman, J. R. (2012). Energy balance and its components: Implications for body weight regulation. Ameri can Journal of Clinical Nutrition, 95, 989-994. https://doi.org/10.3945/ajcn.112.036350

Hare, T. A., Malmaud, J., \& Rangel, A. (2011). Focusing Attention on the Health Aspects of Foods Changes Value Signals in vmPFC and Improves Dietary Choice. Journal of Neuroscience ,31(30), 11077-11087. https://doi.org/10.1523/JNEUROSCI.6383-10.2011 
Haynes, J.-D. (2015). A Primer on Pattern-Based Approaches to fMRI: Principles, Pitfalls, and Perspectives. Neuron, 87(2), 257-270. https://doi.org/10.1016/j.neuron.2015.05.025

Hege, M. A., Veit, R., Krumsiek, J., Kullmann, S., Heni, M., Rogers, P. J., Brunstrom, J. M., Fritsche, A., \& Preissl, H. (2018). Eating less or more - Mindset induced changes in neural correlates of pre-meal planning. Appetite, 125, 492-501. https://doi.org/10.1016/j. appet.2018.03.006

Herman, C. P., \& Polivy, J. (1980). Restrained Eating. In A. J. Skunard (Ed.), Obesity (pp. 208-225). Saunders: Philadelphia.

Hermann, P., Gál, V., Kóbor, I., Kirwan, C. B., Kovács, P., Kitka, T., Lengyel, Z., Bálint, E., Varga, B., Csekő, C., \& Vidnyánszky, Z. (2019). Efficacy of weight loss intervention can be predicted based on early alterations of fMRI food cue reactivity in the striatum. Neurolmage: Clinical, 23(October 2018), 101803. https://doi.org/10.1016/j.nicl. 2019.101803

Heron, K. E., \& Smyth, J. M. (2010). Ecological momentary interventions: incorporating mobile technology into psychosocial and health behaviour treatments. British Journal of Health Psychology, 15(Pt 1), 1-39. https://doi.org/10.1348/135910709X466063

Hill, J. O., Peters, J. C. (1998). Environmental Contributions to the Obesity Epidemic. Science, 280(5368), 1371-1374. https://doi.org/10.1126/science.280.5368.1371

Hill, J. O., Wyatt, H. R., \& Melanson, E. L. (2000). Genetic and environmental contributions to obesity. The Medical Clinics of North America, 84(2), 333-346. https://doi.org/Doi 10.1016/S0025-7125(05)70224-8

Hogenkamp, P. S., Cedernaes, J., Chapman, C. D., Vogel, H., Hjorth, O. C., Zarei, S., Lundberg, L. S., Brooks, S. J., Dickson, S. L., Benedict, C., \& Schiöth, H. B. (2013). Calorie anticipation alters food intake after low-caloric not high-caloric preloads. Obesity, 21(8), 1548-1553. https://doi.org/10.1002/oby.20293

Hollmann, M., Hellrung, L., Pleger, B., Schlögl, H., Kabisch, S., Stumvoll, M., Villringer, A., \& Horstmann, A. (2012). Neural correlates of the volitional regulation of the desire for food. International Journal of Obesity, 36(5), 648-655. https://doi.org/10.1038/ijo. 2011.125

Hu, F. B., \& Malik, V. S. (2010). Sugar-sweetened beverages and risk of obesity and type 2 diabetes: Epidemiologic evidence. Physiology \& Behavior, 100(1), 47-54. https://doi. org/10.1016/j.physbeh.2010.01.036

Hubacher, M., Kappos, L., Weier, K., Stöcklin, M., Opwis, K., \& Penner, I.-K. (2015). Case-Based fMRI Analysis after Cognitive Rehabilitation in MS: A Novel Approach. Frontiers in Neurology, 6(MAR), 1-8. https://doi.org/10.3389/fneur.2015.00078

Hussain, S. S., \& Bloom, S. R. (2013). The regulation of food intake by the gut-brain axis: implications for obesity. International Journal of Obesity, 37(5), 625-633. https://doi. org/10.1038/ijo.2012.93 
Imamura, F., O'Connor, L., Ye, Z., Mursu, J., Hayashino, Y., Bhupathiraju, S. N., \& Forouhi, N. G. (2015). Consumption of sugar sweetened beverages, artificially sweetened beverages, and fruit juice and incidence of type 2 diabetes: systematic review, meta-a nalysis, and estimation of population attributable fraction. BMJ, h3576. https://doi. org/10.1136/bmj.h3576

Jansen, A. (1998). A learning model of binge eating: Cue reactivity and cue exposure. Behaviour Research and Therapy, 36, 257-272. https://doi.org/10.1016/S0005-7967(98)00055-2

Jansen, A., Havermans, R. C., \& Nederkoorn, C. (2011). Cued Overeating. In V. R. Preedy, R. R. Watson, \& C. R. Martin (Eds.), Handbook of Behavior, Food and Nutrition (pp. 14311443). Springer New York. https://doi.org/10.1007/978-0-387-92271-3

Jansen, A., Houben, K., \& Roefs, A. (2015). A Cognitive Profile of Obesity and Its Translation into New Interventions. Frontiers in Psychology, 6(NOV), 1-9. https://doi.org/10.3389/ fpsyg.2015.01807

Jansen, A., Schyns, G., Bongers, P., \& van den Akker, K. (2016). From lab to clinic: Extinction of cued cravings to reduce overeating. Physiology and Behavior, 162, 174-180. https:// doi.org/10.1016/j.physbeh.2016.03.018

Jansen, A., Theunissen, N., Slechten, K., Nederkoorn, C., Boon, B., Mulkens, S., \& Roefs, A. (2003). Overweight children overeat after exposure to food cues. Eating Behaviors, 4(2), 197209. https://doi.org/10.1016/S1471-0153(03)00011-4

Janssen, L. K., Herzog, N., Waltmann, M., Breuer, N., Wiencke, K., Rausch, F., Hartmann, H., Poessel, M., \& Horstmann, A. (2019). Lost in Translation? On the Need for Convergence in Animal and Human Studies on the Role of Dopamine in Diet-Induced Obesity. Current Addiction Reports. https://doi.org/10.1007/s40429-019-00268-w

Just, T., Pau, H. W., Engel, U., \& Hummel, T. (2008). Cephalic phase insulin release in healthy humans after taste stimulation? Appetite, 51(3), 622-627. https://doi.org/10.1016/j. appet.2008.04.271

Kahnt, T. (2018). A decade of decoding reward-related fMRI signals and where we go from here. Neurolmage, 180(June 2017), 324-333. https://doi.org/10.1016/j.neuroimage. 2017.03 .067

Kahnt, T., Park, S. Q., Haynes, J.-D., \& Tobler, P. N. (2014). Disentangling neural representations of value and salience in the human brain. Proceedings of the National Academy of Sciences, 111(13), 5000-5005. https://doi.org/10.1073/pnas.1320189111

Kober, H., Mende-Siedlecki, P., Kross, E. F., Weber, J., Mischel, W., Hart, C. L., \& Ochsner, K. N. (2010). Prefrontal-striatal pathway underlies cognitive regulation of craving. Pro ceedings of the National Academy of Sciences, 107(33), 14811-14816. https://doi. org/10.1073/pnas.1007779107

Kojima, M., Hosoda, H., Date, Y., Nakazato, M., Matsuo, H., \& Kangawa, K. (1999). Ghrelin is a growth-hormone-releasing acylated peptide from stomach. Nature, 402(6762), 656660. https://doi.org/10.1038/45230 
Kriegeskorte, N., Goebel, R., \& Bandettini, P. (2006). Information-based functional brain mapping. Proceedings of the National Academy of Sciences, 103(10), 3863-3868.https:// doi.org/10.1073/pnas.0600244103

Kriegeskorte, N., Simmons, W. K., Bellgowan, P. S. F., \& Baker, C. I. (2009). Circular analysis in systems neuroscience : the dangers of double dipping. Nature Neuroscience, 12(5), 535-540. https://doi.org/10.1038/nn.2303

Kühn, S., \& Gallinat, J. (2013). Does Taste Matter? How Anticipation of Cola Brands Influences Gustatory Processing in the Brain. PLoS ONE, 8(4), e61569. https://doi.org/10.1371/ journal.pone.0061569

Leigh, S. J., \& Morris, M. J. (2018). The role of reward circuitry and food addiction in the obesity epidemic: An update. Biological Psychology, 131, 31-42. https://doi.org/10.1016/j. biopsycho.2016.12.013

Lim, J., Wood, A., \& Green, B. G. (2009). Derivation and Evaluation of a Labeled Hedonic Scale. Chemical Senses, 34(9), 739-751. https://doi.org/10.1093/chemse/bjp054

Lowe, M. R., \& Butryn, M. L. (2007). Hedonic hunger: A new dimension of appetite? Physiology \& Behavior, 91(4), 432-439. https://doi.org/10.1016/j.physbeh.2007.04.006

Martin, L. E., Holsen, L. M., Chambers, R. J., Bruce, A. S., Brooks, W. M., Zarcone, J. R., Butler, M. G., \& Savage, C. R. (2010). Neural Mechanisms Associated With Food Motivation in Obese and Healthy Weight Adults. Obesity, 18(2), 254-260. https://doi.org/10.1038/ oby. 2009.220

McCann, M. T., Wallace, J. M. W., Robson, P. J., Rennie, K. L., McCaffrey, T. A., Welch, R. W., \& Livingstone, M. B. E. (2013). Influence of nutrition labelling on food portion size consumption. Appetite, 65, 153-158. https://doi.org/10.1016/j.appet.2013.02.013

Miedl, S. F., Blechert, J., Meule, A., Richard, A., \& Wilhelm, F. H. (2018). Suppressing images of desire: Neural correlates of chocolate-related thoughts in high and low trait chocolate cravers. Appetite, 126, 128-136. https://doi.org/10.1016/j.appet.2018.03. 004

Mitchell, N. S., Catenacci, V. A., Wyatt, H. R., \& Hill, J. O. (2011). Obesity: Overview of an Epidemic. Psychiatric Clinics of North America, 34(4), 717-732. https://doi.org/10.1016/j.psc. 2011.08.005

Moeller, S., Yacoub, E., Olman, C. A., Auerbach, E., Strupp, J., Harel, N., \& Uğurbil, K. (2010). Multiband multislice GE-EPI at 7 tesla, with 16-fold acceleration using partial parallel imaging with application to high spatial and temporal whole-brain fMRI. Magnetic Resonance in Medicine, 63(5), 1144-1153. https://doi.org/10.1002/mrm.22361

Morton, G. J., Cummings, D. E., Baskin, D. G., Barsh, G. S., \& Schwartz, M. W. (2006). Central nervous system control of food intake and body weight. Nature, 443(7109), 289-295. https://doi.org/10.1038/nature05026

Mumford, J. A., \& Nichols, T. E. (2008). Power calculation for group fMRI studies accounting for arbitrary design and temporal autocorrelation. Neurolmage, 39(1), 261-268. https://doi.org/10.1016/j.neuroimage.2007.07.061 
Mumford, J. A., Turner, B. O., Ashby, F. G., \& Poldrack, R. A. (2012). Deconvolving BOLD activation in event-related designs for multivoxel pattern classification analyses. Neurolmage, 59(3), 2636-2643. https://doi.org/10.1016/j.neuroimage.2011.08.076

Mur, M., Bandettini, P. A., \& Kriegeskorte, N. (2009). Revealing representational content with pattern-information fMRI--an introductory guide. Social Cognitive and Affective Neuroscience, 4(1), 101-109. https://doi.org/10.1093/scan/nsn044

Murphy, K. G., \& Bloom, S. R. (2006). Gut hormones and the regulation of energy homeostasis. Nature, 444(7121), 854-859. https://doi.org/10.1038/nature05484

Murphy, K. G., Dhillo, W. S., \& Bloom, S. R. (2006). Gut Peptides in the Regulation of Food Intake and Energy Homeostasis. Endocrine Reviews, 27(7), 719-727. https://doi.org/10.1210/ er.2006-0028

Ng, J., Stice, E., Yokum, S., \& Bohon, C. (2011). An fMRI study of obesity, food reward, and perceived caloric density. Does a low-fat label make food less appealing? Appetite, 57(1), 65-72. https://doi.org/10.1016/j.appet.2011.03.017

Norman, K. a, Polyn, S. M., Detre, G. J., \& Haxby, J. V. (2006). Beyond mind-reading: multi-voxel pattern analysis of fMRI data. Trends in Cognitive Sciences, 10(9), 424-430. https://doi. org/10.1016/j.tics.2006.07.005

O'Rahilly, S., \& Farooqi, I. S. (2006). Genetics of obesity. Philosophical Transactions of the Royal Society B: Biological Sciences, 361(1471), 1095-1105. https://doi.org/10.1098/ rstb.2006.1850

Oosterhof, N. N., Connolly, A. C., \& Haxby, J. V. (2016). CoSMoMVPA: Multi-Modal Multivariate Pattern Analysis of Neuroimaging Data in Matlab/GNU Octave. Frontiers in Neuroinformatics, 10(July), 1-27. https://doi.org/10.3389/fninf.2016.00027

Peelen, M. V., \& Caramazza, A. (2012). Conceptual Object Representations in Human Anterior Temporal Cortex. Journal of Neuroscience, 32(45), 15728-15736. https://doi.org/10. 1523/JNEUROSCI.1953-12.2012

Pepino, M. Y. (2015). Metabolic effects of non-nutritive sweeteners. Physiology \& Behavior, 152, 450-455. https://doi.org/10.1016/j.physbeh.2015.06.024

Perello, M., \& Dickson, S. L. (2014). Ghrelin signaling on food reward: a salient link between the gut and the mesolimbic system. Journal of Neuroendocrinology. https://doi. org/10.1111/jne.12236

Pfabigan, D., Seidel, E., Sladky, R., \& Hahn, A. (2014). P300 amplitude variation is related to ven tral striatum BOLD response during gain and loss anticipation: An EEG and fMRI experiment. Neurolmage, 96, 12-21. https://doi.org/10.1016/j.neuroimage.2014. 03.077

Plassmann, H., O'Doherty, J., Shiv, B., \& Rangel, A. (2008). Marketing actions can modulate neural representations of experienced pleasantness. Proceedings of the National Academy of Sciences of the United States of America, 105(3), 1050-1054. https://doi.org/ 10.1073/pnas.0706929105 
Pohl, T. M., Tempelmann, C., \& Noesselt, T. (2017). How task demands shape brain responses to visual food cues. Human Brain Mapping, 38(6), 2897-2912. https://doi.org/10.1002 /hbm.23560

Poldrack, R. A. (2011). Inferring mental states from neuroimaging data: From reverse inference to large-scale decoding. Neuron, 72(5), 692-697. https://doi.org/10.1016/j.neuron. 2011.11.001

Provencher, V., \& Jacob, R. (2016). Impact of Perceived Healthiness of Food on Food Choices and Intake. Current Obesity Reports, 5(1), 65-71. https://doi.org/10.1007/s13679-0160192-0

Pujol, J., Blanco-Hinojo, L., Coronas, R., Esteba-Castillo, S., Rigla, M., Martínez-Vilavella, G., Deus, J., Novell, R., \& Caixàs, A. (2018). Mapping the sequence of brain events in response to disgusting food. Human Brain Mapping, 39(1), 369-380. https://doi.org/10.1002/ hbm.23848

Pursey, K. M., Stanwell, P., Callister, R. J., Brain, K., Collins, C. E., \& Burrows, T. L. (2014). Neural Responses to Visual Food Cues According to Weight Status: A Systematic Review of Functional Magnetic Resonance Imaging Studies. Frontiers in Nutrition, 1(July), 1-11. https://doi.org/10.3389/fnut.2014.00007

Quatela, A., Callister, R., Patterson, A., \& MacDonald-Wicks, L. (2016). The Energy Content and Composition of Meals Consumed after an Overnight Fast and Their Effects on Diet Induced Thermogenesis: A Systematic Review, Meta-Analyses and Meta-Regressions. Nutrients, 8(11), 670. https://doi.org/10.3390/nu8110670

Ravussin, E., \& Bogardus, C. (2000). Energy balance and weight regulation: genetics versus environment. British Journal of Nutrition, 83(S1), S17-S20. https://doi.org/10.1017/ S0007114500000908

Reader, S. W., Lopez, R. B., \& Denny, B. T. (2018). Cognitive reappraisal of low-calorie food predicts real-world craving and consumption of high- and low-calorie foods in daily life. Appetite, 131(May), 44-52. https://doi.org/10.1016/j.appet.2018.08.036

Reed, G. W., \& Hill, J. O. (1996). Measuring the thermic effect of food. The American Journal of Clinical Nutrition, 63(2), 164-169. https://doi.org/10.1093/ajcn/63.2.164

Rhea, E. M., Salameh, T. S., Gray, S., Niu, J., Banks, W. A., \& Tong, J. (2018). Ghrelin transport across the blood-brain barrier can occur independently of the growth hormone secretagogue receptor. Molecular Metabolism, 18(2018), 88-96. https://doi.org/10. 1016/j.molmet.2018.09.007

Robinson, E., Haynes, A., Hardman, C. A., Kemps, E., Higgs, S., \& Jones, A. (2017). The bogus taste test: Validity as a measure of laboratory food intake. Appetite, 116, 223-231. https://doi.org/10.1016/j.appet.2017.05.002

Roefs, A., Franssen, S., \& Jansen, A. (2018). The dynamic nature of food reward processing in the brain. Current Opinion in Clinical Nutrition and Metabolic Care, 21(6), 444-448. https://doi.org/10.1097/MCO.0000000000000504 
Roefs, A., Houben, K., \& Werthmann, J. Desire for food and the power of mind. In Intergovernmental Panel on Climate Change (Ed.), The psychology of desire (pp. 323346). Cambridge University Press. https://doi.org/10.1128/AAC.03728-14

Roefs, A., \& Jansen, A. (2004). The effect of information about fat content on food consumption in overweight/obese and lean people. Appetite, 43(3), 319-322. https://doi.org/10. 1016/j.appet.2004.05.002

Roiser, J. P., Linden, D. E., Gorno-Tempinin, M. L., Moran, R. J., Dickerson, B. C., \& Grafton, S. T. (2016). Minimum statistical standards for submissions to Neuroimage: Clinical. Neurolmage: Clinical, 12, 1045-1047. https://doi.org/10.1016/j.nicl.2016.08.002

Romo-Romo, A., Aguilar-Salinas, C. A., Brito-C?rdova, G. X., G?mez D?az, R. A., Vilchis Valent?n, D., \& Almeda-Valdes, P. (2016). Effects of the Non-Nutritive Sweeteners on Glucose Metabolism and Appetite Regulating Hormones: Systematic Review of Observational Prospective Studies and Clinical Trials. PLOS ONE, 11(8), e0161264. https://doi.org/10. 1371/journal.pone.0161264

Rothemund, Y., Preuschhof, C., Bohner, G., Bauknecht, H.-C., Klingebiel, R., Flor, H., \& Klapp, B. F. (2007). Differential activation of the dorsal striatum by high-calorie visual food stimuli in obese individuals. Neurolmage, 37(2), 410-421. https://doi.org/10.1016/j. neuroimage.2007.05.008

Rothkirch, M., Schmack, K., Schlagenhauf, F., \& Sterzer, P. (2012). Implicit motivational value and salience are processed in distinct areas of orbitofrontal cortex. Neurolmage, 62, 1717-1725. https://doi.org/10.1016/j.neuroimage.2012.06.016

Salamone, J. D., \& Correa, M. (2012). The mysterious motivational functions of mesolimbic dopamine. Neuron, 76(3), 470-485. https://doi.org/10.1016/j.neuron.2012.10.021

Scharmüller, W., Übel, S., Ebner, F., \& Schienle, A. (2012). Appetite regulation during food cue exposure: a comparison of normal-weight and obese women. Neuroscience Letters, 518(2), 106-110. https://doi.org/10.1016/j.neulet.2012.04.063

Schienle, A., Schäfer, A., Hermann, A., \& Vaitl, D. (2009). Binge-Eating Disorder: Reward Sensitivity and Brain Activation to Images of Food. Biological Psychiatry, 65(8), 654-661. https://doi.org/10.1016/j.biopsych.2008.09.028

Schneider, K., Dogil, G., \& Möbius, B. (2011). Reaction time and decision difficulty in the perception of intonation. Proceedings of the Annual Conference of the International speech Communication Association, INTERSPEECH, August, 2221-2224.

Schroder, H. S., Moran, T. P., Donnellan, M. B., \& Moser, J. S. (2014). Mindset induction effects on cognitive control: A neurobehavioral investigation. Biological Psychology, 103, $27-$ 37. https://doi.org/10.1016/j.biopsycho.2014.08.004

Schwartz, M., Woods, S. C., \& Porte, D. (2000). Central nervous system control of food intake. Nature, 661-671. http://www.sciencedirect.com/science/article/pii/S0065280608601 526/pdf?md5=06864451afe498a9caef5711373d907d\&pid=1-s2.0-S0065280608601 526-main.pdf\&_valck=1 
Schyns, G., Roefs, A., \& Jansen, A. (2020). Tackling sabotaging cognitive processes to reduce overeating; expectancy violation during food cue exposure. Physiology and Behavior, 222(April), 112924. https://doi.org/10.1016/j.physbeh.2020.112924

Schyns, G., Roefs, A., Mulkens, S., \& Jansen, A. (2016). Expectancy violation, reduction of food cue reactivity and less eating in the absence of hunger after one food cue exposure session for overweight and obese women. Behaviour Research and Therapy, 76, 5764. https://doi.org/10.1016/j.brat.2015.11.007

Schyns, G., van den Akker, K., Roefs, A., Hilberath, R., \& Jansen, A. (2018). What works better? Food cue exposure aiming at the habituation of eating desires or food cue exposure aiming at the violation of overeating expectancies? Behaviour Research and Therapy, 102(December 2017), 1-7. https://doi.org/10.1016/j.brat.2017.12.001

Schyns, G., van den Akker, K., Roefs, A., Houben, K., \& Jansen, A. (2019). Exposure therapy vs lifestyle intervention to reduce food cue reactivity and binge eating in obesity: A pilot study. Journal of Behavior Therapy and Experimental Psychiatry, November 2018, 1014 53. https://doi.org/10.1016/j.jbtep.2019.01.005

Scott, K. M., Bruffaerts, R., Simon, G. E., Alonso, J., Angermeyer, M., de Girolamo, G., Demyttenaere, K., Gasquet, I., Haro, J. M., Karam, E., Kessler, R. C., Levinson, D., Medina Mora, M. E., Oakley Browne, M. A., Ormel, J., Villa, J. P., Uda, H., \& Von Korff, M. (2008). Obesity and mental disorders in the general population: results from the world mental health surveys. International Journal of Obesity, 32(1), 192-200. https:// doi.org/10.1038/sj.ijo.0803701

Setsompop, K., Gagoski, B. A., Polimeni, J. R., Witzel, T., Wedeen, V. J., \& Wald, L. L. (2012). Blipped-controlled aliasing in parallel imaging for simultaneous multislice echo pla nar imaging with reduced g-factor penalty. Magnetic Resonance in Medicine, 67(5), 1210-1224. https://doi.org/10.1002/mrm.23097

Shide, D. J., \& Rolls, B. J. (1995). Information about the fat content of preloads influences energy intake in healthy women. Journal of the American Dietetic Association, 95, 993-998. https://doi.org/10.1016/S0002-8223(95)00273-1

Siep, N., Roefs, A., Roebroeck, A., Havermans, R., Bonte, M., \& Jansen, A. (2012). Fighting food temptations: The modulating effects of short-term cognitive reappraisal, suppression and up-regulation on mesocorticolimbic activity related to appetitive motivation. Neurolmage, 60(1), 213-220. https://doi.org/10.1016/j.neuroimage.2011.12.067

Silventoinen, K., \& Kaprio, J. (2009). Genetics of Tracking of Body Mass Index from Birth to Late Middle Age: Evidence from Twin and Family Studies. Obesity Facts, 2(3), 196-202. https://doi.org/10.1159/000219675

Small, D M. (2009). Individual differences in the neurophysiology of reward and the obesity epidemic. International Journal of Obesity, 33, S44-S48. https://doi.org/10.1038/ijo. 2009.71 
Small, Dana M., Veldhuizen, M. G., Felsted, J., Mak, Y. E., \& McGlone, F. (2008). Separable Substrates for Anticipatory and Consummatory Food Chemosensation. Neuron, 57(5), 786-797. https://doi.org/10.1016/j.neuron.2008.01.021

Smeets, P. a. M., Charbonnier, L., van Meer, F., van der Laan, L. N., \& Spetter, M. S. (2012). Food-induced brain responses and eating behaviour. Proceedings of the Nutrition Society, 71(04), 511-520. https://doi.org/10.1017/S0029665112000808

Stice, E., Yokum, S., Blum, K., \& Bohon, C. (2010). Weight Gain Is Associated with Reduced Striatal Response to Palatable Food. Journal of Neuroscience, 30(39), 13105-13109. https://doi.org/10.1523/JNEUROSCI.2105-10.2010

Stice, Eric, Figlewicz, D. P., Gosnell, B. a., Levine, A. S., \& Pratt, W. E. (2013). The contribution of brain reward circuits to the obesity epidemic. Neuroscience and Biobehavioral Reviews, 37(9), 2047-2058. https://doi.org/10.1016/j.neubiorev.2012.12.001

Stice, Eric, Spoor, S., Bohon, C., Veldhuizen, M. G., \& Small, D. M. (2008). Relation of reward from food intake and anticipated food intake to obesity: A functional magnetic resonance imaging study. Journal of Abnormal Psychology, 117(4), 924-935. https://doi.org/10. $1037 / a 0013600$

Stoeckel, L. E., Weller, R. E., Cook, E. W., Twieg, D. B., Knowlton, R. C., \& Cox, J. E. (2008). Widespread reward-system activation in obese women in response to pictures of high-calorie foods. Neurolmage, 41(2), 636-647. https://doi.org/10.1016/j.neuroim age.2008.02.031

Sun, X., Veldhuizen, M. G., Wray, A. E., de Araujo, I. E., Sherwin, R. S., Sinha, R., \& Small, D. M. (2014). The neural signature of satiation is associated with ghrelin response and triglyceride metabolism. Physiology \& Behavior, 136, 63-73. https://doi.org/10.1016/j. physbeh.2014.04.017

Suzuki, S., Cross, L., \& O'Doherty, J. P. (2017). Elucidating the underlying components of food valuation in the human orbitofrontal cortex. Nature Neuroscience, 20. https://doi.org/ 10.1038/s41593-017-0008-x

Swinburn, B. a., Sacks, G., Hall, K. D., McPherson, K., Finegood, D. T., Moodie, M. L., \& Gortmaker, S. L. (2011). The global obesity pandemic: Shaped by global drivers and local environments. The Lancet, 378(9793), 804-814. https://doi.org/10.1016/S01406736(11)60813-1

Swithers, S. E., Martin, A. A., \& Davidson, T. L. (2010). High-intensity sweeteners and energy balance. Physiology \& Behavior, 100(1), 55-62. https://doi.org/10.1016/j.physbeh. 2009.12.021

Tenan, M. S., Bohannon, A. W., Macfarlane, D. J., \& Crouter, S. E. (2018). Determining day-to-day human variation in indirect calorimetry using Bayesian decision theory. Experimental Physiology, 103(12), 1579-1585. https://doi.org/10.1113/EP087115 
Tetley, A., Brunstrom, J., \& Griffiths, P. (2009). Individual differences in food-cue reactivity. The role of BMI and everyday portion-size selections. Appetite, 52(3), 614-620. https://doi.org/10.1016/j.appet.2009.02.005

Thomas, J. M., Higgs, S., Dourish, C. T., Hansen, P. C., Harmer, C. J., \& McCabe, C. (2015). Satia tion attenuates BOLD activity in brain regions involved in reward and increases a ctivity in dorsolateral prefrontal cortex: an fMRI study in healthy volunteers. The American Journal of Clinical Nutrition, 101(4), 697-704. https://doi.org/10.3945/ajcn. 114.097543

van Bloemendaal, L., Veltman, D. J., ten Kulve, J. S., Groot, P. F. C., Ruhé, H. G., Barkhof, F., Sloan, J. H., Diamant, M., \& ljzerman, R. G. (2015). Brain reward-system activation in response to anticipation and consumption of palatable food is altered by glucagonlike peptide-1 receptor activation in humans. Diabetes, Obesity and Metabolism, 17(9), 878-886. https://doi.org/10.1111/dom.12506

van den Akker, K., Havermans, R. C., Bouton, M. E., \& Jansen, A. (2014). How partial reinforce ment of food cues affects the extinction and reacquisition of appetitive responses. A new model for dieting success? Appetite, 81, 242-252. https://doi.org/10.1016/j. appet.2014.06.024

van den Akker, K., Schyns, G., \& Jansen, A. (2016). Enhancing inhibitory learning to reduce overeating: Design and rationale of a cue exposure therapy trial in overweight and obese women. Contemporary Clinical Trials, 49, 85-91. https://doi.org/10.1016/j.cct. 2016.06.008

van den Akker, K., Stewart, K., Antoniou, E. E., Palmberg, A., \& Jansen, A. (2014). Food Cue Reactivity, Obesity, and Impulsivity: Are They Associated? Current Addiction Reports, 1(4), 301-308. https://doi.org/10.1007/s40429-014-0038-3

van der Laan, L. N., de Ridder, D. T. D., Viergever, M. A., \& Smeets, P. A. M. (2011). The first taste is always with the eyes: a meta-analysis on the neural correlates of processing visual food cues. Neurolmage, 55(1), 296-303. https://doi.org/10.1016/j.neuroim age.2010.11.055

Veldhuizen, Maria G., Nachtigal, D. J., Flammer, L. J., de Araujo, I. E., \& Small, D. M. (2013). Verbal descriptors influence hypothalamic response to low-calorie drinks. Molecular Metabolism, 2(3), 270-280. https://doi.org/10.1016/j.molmet.2013.06.004

Veldhuizen, Maria Geraldine, Babbs, R. K., Patel, B., Fobbs, W., Kroemer, N. B., Garcia, E., Yeomans, M. R., \& Small, D. M. (2017). Integration of Sweet Taste and Metabolism Determines Carbohydrate Reward. Current Biology, 27(16), 2476-2485.e6. https://doi. org/10.1016/j.cub.2017.07.018

Volkow, N. D., Wang, G.-J., Tomasi, D., \& Baler, R. D. (2013). Obesity and addiction: neurobiological overlaps. Obesity Reviews, 14(1), 2-18. https://doi.org/10.1111/j.1467789X.2012.01031.x 
Volkow, Nora D., Wang, G.-J., \& Baler, R. D. (2011). Reward, dopamine and the control of food intake: implications for obesity. Trends in Cognitive Sciences, 15(1), 37-46. https://doi. org/10.1016/j.tics.2010.11.001

Vrang, N., \& Larsen, P. J. (2010). Preproglucagon derived peptides GLP-1, GLP-2 and oxyntomodulin in the CNS: Role of peripherally secreted and centrally produced peptides. Progress in Neurobiology, 92(3), 442-462. https://doi.org/10.1016/j. pneurobio.2010.07.003

Weir, J. B. de V. (1949). New methods for calculating metabolic rate with special reference to protein metabolism. The Journal of Physiology, 109(1-2), 1-9. https://doi.org/10.1113/ jphysiol.1949.sp004363

Werthmann, J., Jansen, A., \& Roefs, A. (2016). Make up your mind about food: A healthy mindset attenuates attention for high-calorie food in restrained eaters. Appetite, 105, 53-59. https://doi.org/10.1016/j.appet.2016.05.005

Wing, R. R., \& Phelan, S. (2005). Long-term weight loss maintenance. The American Journal of Clinical Nutrition, 82(3), 222-225. https://doi.org/2005;82(suppl):222S-5S

Woo, C.-W., Krishnan, A., \& Wager, T. D. (2014). Cluster-extent based thresholding in fMRI analyses: Pitfalls and recommendations. Neurolmage, 91, 412-419. https://doi.org/ 10.1016/j.neuroimage.2013.12.058

Wooley, S. C. (1972). Physiologic versus cognitive factors in short term food regulation in the obese and nonobese. Psychosomatic Medicine, 34(1), 62-68.

World Health Organization. (2017). Obesity and overweight. http://www.who.int/mediacentre/ factsheets/fs311/en/

World Health Organization. (2020). Obesity and overweight. http://www.who.int/en/news-room/ fact-sheets/detail/obesity-and-overweight

Xu, J., Moeller, S., Auerbach, E. J., Strupp, J., Smith, S. M., Feinberg, D. A., Yacoub, E., \& Uğurbil, K. (2013). Evaluation of slice accelerations using multiband echo planar imaging at 3T. Neurolmage, 83, 991-1001. https://doi.org/10.1016/j.neuroimage.2013.07.055

Yeshurun, Y., Swanson, S., Simony, E., Chen, J., Lazaridi, C., Honey, C. J., \& Hasson, U. (2017). Same Story, Different Story. Psychological Science, 28(3), 307-319. https://doi. org/10.1177/0956797616682029

Yokum, S., Ng, J., \& Stice, E. (2011). Attentional Bias to Food Images Associated With Elevated Weight and Future Weight Gain: An fMRI Study. Obesity, 19(9), 1775-1783. https://doi. org/10.1038/oby.2011.168

Yokum, S., \& Stice, E. (2013). Cognitive regulation of food craving: effects of three cognitive reappraisal strategies on neural response to palatable foods. International Journal of 
SAMENVATTING 
Wereldwijd is de prevalentie van obesitas hoog. Obesitas gaat gepaard met een hoog risico op gezondheidsproblemen, zoals hart en vaatziekten, diabetes en sommige typen kanker. Blijvend afvallen en gezonder gaan leven blijven moeilijk. Gewichtstoename wordt veroorzaakt door een langdurige energie disbalans: de energie-inname (aantal kilocalorieën) overstijgt de hoeveelheid energie die verbrand wordt. Een oorzaak voor deze disbalans wordt gezocht in de huidige "obesogene omgeving"; overal is lekkere calorierijke voeding in overvloed verkrijgbaar. Maar, hoewel we allemaal in deze obesogene omgeving leven, is niet iedereen te zwaar. Een veronderstelling is dat mensen met overgewicht wellicht gevoeliger zijn voor deze verleidingen. Gevoeliger betekent dat mensen sterker op calorierijke voeding en/ of de omgeving van calorierijke voeding reageren door onder andere: meer trek te ervaren, meer honger-gerelateerde hormonen te produceren of sterkere activatie in beloningsgebieden van de hersenen te hebben. Deze zogeheten food cue reactiviteit zorgt ervoor dat het lichaam zich voorbereid op (overmatige) voedselinname. Daarbij heeft calorierijke voeding twee gezichten, aan de ene kant een hoog hedonische waarde (het is lekker en belonend) en aan de andere kant een ongezond waarde (veel calorieën, risico van gewichtstoename). De manier waarop mensen omgaan met de verleidingen van calorierijke voeding lijkt niet altijd hetzelfde en hangt mogelijk samen met iemands huidige mindset. Een mindset wordt gedefinieerd als de gedachtes, overtuigingen, assumpties en verwachtingen die iemand heeft over een onderwerp. Deze mindsets kunnen dynamisch zijn, wat betekend dat iemand kan variëren afhankelijk van iemands emotionele staat of van de situatie. Het onderzoeksdoel van dit proefschrift is om de invloed van mindset op psychologische en fysiologische variabelen betrokken bij eetgedrag te onderzoeken.

Hoofdstuk 1 introduceert de invloed van mindset op eetgedrag, cognitie, neurale en hormonale responsen en metabolisme. Mindset wordt in dit proefschrift geoperationaliseerd als een verlies van controle versus een in controle mindset (hoofdstuk 3), een hedonisme aandacht-focus versus een neutrale aandacht-focus (hoofdstuk 2 en 5) en een hoog-, midden-, en laagcalorisch label (hoofdstuk 4). De verschillende afhankelijkevariabelen die gebruiktzijn in dit proefschriftzijn: hoeveelheid eten in hoofdstuk 3, subjectieve beleving (bijv: zin om te eten, lekker vinden, zelfcontrole, hongergevoel en verzadiging) in hoofdstuk 3 en 4, neurale responsen (activiteit in mesocorticolimbische en controle gerelateerde hersengebieden gemeten met een MRI-scanner) in hoofdstuk 2, 3 en 5; spijsverteringshormonen gerelateerd aan honger (ghreline) en verzadiging (glucagon-like peptide 1; GLP-1) in hoofdstuk 
3; en metabolisme (door berekening van het thermische effect van voeding uit rustmetabolisme) in hoofdstuk 4. Daarbij, introduceren we een mogelijk effect van food cue exposure therapie in vergelijking tot een lifestyle interventie op neurale responsen voor en na interventie in hoofdstuk 5.

In hoofdstuk 2 wordt de invloed van een aandachtsfocus (hedonisch versus neutraal) op hersenactiviteit onderzocht terwijl vrouwen met overgewicht foto's zien van plaatjes van calorierijk eten die ze of heel erg lekker of helemaal niet lekker vinden. De hersengebieden betrokken bij het verwerken van voedingsstimuli behoren tot het mesocorticolimbische systeem. De functie van het mesocorticolimbische systeem is ambigue, het is namelijk betrokken bij beloningsverwerking en bij motivationele sailliantie. De vraag is dus, welke functie (beloningsverwerking of algemene motivationele sailliantie) hoort bij het verwerken van voedingsstimuli. Om dit te onderzoeken hebben we hebben de functionele MRI-data univariaat geanalyseerd en door middel van een multivoxel pattern analyse (MVPA). De MVPA maakt het mogelijk om, naast het leve/ van activatie, neurale patronen van activatie te onderzoeken. Resultaten lieten zien dat aandachtsfocus die proefpersonen hebben wanneer ze naar voedingsmiddelen kijken erg uitmaakt. Er was meer activiteit in hersengebieden in het mesocorticolimbische systeem wanneer proefpersonen zich richtten op de smaak van de gepresenteerde voedingsmiddelen (hedonische aandachtsfocus) dan wanneer proefpersonen zich richtten op de kleuren van de voedingsmiddelen (neutrale aandachtsfocus). Daarnaast, was er geen significant verschil in hersenactiviteit tussen het zien van heel erg lekkere of helemaal niet lekkere voedingsmiddelen. Dit suggereert dat activiteit in het mesocorticolimbische systeem meer zegt over motivationele saillantie dan over de belonende waarde van calorierijk eten. Een verschil tussen lekker en niet lekkere voedingsmiddelen kan was wel zichtbaar in de multivoxel neurale representaties, en dit verschil was met name zichtbaar wanneer proefpersonen een hedonische aandachtsfocus hadden. Deze bevinding geeft aan dat er dus wel een onderscheid gemaakt kan worden in neurale responsen tussen heel erg lekkere en heel erg niet lekkere voeding en dat dit sterker is wanneer de aandacht gericht is op de smaak, maar alleen wanneer er naar neurale multivoxel patronen gekeken wordt. Daarmee onderstreept dit hoofdstuk hoe sterk de invloed van aandachtsfocus is op hersenprocessen bij het zien van voeding en het belang om daar rekening mee te houden bij fMRI onderzoek.

In hoofdstuk 3 is de invloed van een verlies van controle-mindset versus een in controle-mindset op eetgedrag, hersenactiviteit en spijsverteringshormoonlevels 
(ghreline en GLP-1) onderzocht. De mindsets werden geïnduceerd door een kort filmpje te laten zien aan vrouwen met een gezond gewicht die van chocolade houden. Daarna werd hersenactiviteit gemeten bij het zien van plaatjes van chocolade. Hormoonlevels werden gemeten op vijf verschillende momenten; twee voor en drie na de mindset inductie. Deze werden tegelijk afgenomen met zelfrapportage over zin in chocolade, gevoel van zelfcontrole en honger. Vervolgens werd chocoladeconsumptie gemeten in een zogenaamde chocoladesmaaktest. Alle vrouwen werden in elk van de mindset condities getest. We verwachtten dat een verlies van controlemindset zou resulteren in een hormonale en neurale response ter voorbereiding om (lekker en) veel te eten en dat de in controle-mindset meer in hormonale en neurale responsen van voldoening en verzadiging zou resulteren. Resultaten lieten zien dat mindset de hoeveelheid van eten en de zin om chocolade te eten kan beïnvloeden. De verlies van controle-mindset resulteerde in meer zin in en meer chocolade consumptie dan de in controle-mindset. We vonden geen significant effect van mindset op neurale responsen. Evenals werd er geen significant effect van mindset gevonden op de hormoonlevels en het gevoel van honger en verzadiging. Interessant is om te concluderen is dat een indirecte en niet taak-relevante mindsetmanipulatie al kan resulteren in een verandering van eetgedrag en zin in eten, maar niet in fysiologische veranderingen.

In hoofdstuk 4 hebben we de invloed van verwachte hoeveelheid calorieën op metabolisme en subjectieve beleving (smaak, lekker vinden en hongergevoel) onderzocht na het drinken van gezoete drankjes. Hiervoor werden verschillende drankjes met dezelfde zoetheid en calorieën (112.5 kcal) maar met verschillende calorielabels (laag- midden- en hoogcalorisch) gebruikt. Metabole response werd gemeten door rustmetabolisme voor en na het drinken van de drie zoete drankjes te meten. We verwachtten dat het hoogcalorische label zou resulteren in een verhoogde rustmetabolisme na het drinken van het drankje ten opzichte van een mid- of laagcalorisch label. Resultaten laten zien dat er geen significant effect van verwachte hoeveelheid calorieën was op de subjectieve maten of op metabolisme. Er was uitsluitend een response van het daadwerkelijke aantal calorieën op rustmetabolisme. We vonden een hoger rustmetabolisme na het drinken van het drinken van het drankje ten opzichte van ervoor, onafhankelijk van het label. Deze resultaten suggereren dat rustmetabolisme niet significant beïnvloed kan worden door verwachte hoeveelheid calorieën, maar alleen beïnvloedbaar is door daadwerkelijke calorie-inname. 
In hoofdstuk 5 hebben we het effect van food cue exposure therapie in vergelijking tot een controle lifestyle interventie op neurale responsen onderzocht. Bij food cue exposure therapie werden proefpersonen in meerdere sessies blootgesteld aan hun favoriete calorierijke voeding (door het zien en ruiken aan de voeding), maar mochten ze het vervolgens niet eten. Dit met als doel om food cue reactiviteit zwakker te maken en daarmee minder trek te hebben wanneer ze hun favoriete calorierijke voeding zien. Voor dit onderzoek zijn neurale responses bij het zien van geïndividualiseerde calorierijke lekkere voeding voor en na behandeling vergeleken bij tien vrouwen met overgewicht (vijf in cue exposure conditie en vijf in de lifestyle interventie). Deze werden geanalyseerd als individuele cases. We verwachtten een sterkere reductie in hersenactiviteit in hersengebieden gerelateerd aan food cue reactiviteit na een succesvolle food cue exposure therapie in vergelijking met de lifestyle interventie. Tegen verwacht in lieten onze resultaten geen reductie zien in de verwachte hersengebieden na food cue exposure therapie maar wel na een lifestyle interventie. Het betrokken mechanisme van food cue exposure is dat de voeding cue een nieuwe associatie krijgt, namelijk bij het zien ervan om het niet te eten. Belangrijk is dat de eerdere associatie (wel eten) niet verdwijnt, maar dat er een nieuwe inhibitie associatie is geleerd. Dit leermechanisme is erg sterk afhankelijk van context (bijv. omgeving, tijd, maar ook: type voeding). Om die reden zouden deze onverwachte neurale bevindingen verklaard kunnen worden door contextuele verschillen tussen de therapie en de scansessies in het experiment.

In hoofdstuk 6 zijn alle bevindingen samengevat en bediscussieerd. Samengevat hebben we aangetoond dat mindset hersenactiviteit en zin in eten bij het zien van calorierijke voeding kan beïnvloeden, maar alleen als de manipulatie sterk en taakgericht is. Mindset kan ook eetgedrag beïnvloeden, maar niet de hormonale of metabole responsen na het eten en de hierbij horende subjectieve belevingen. Daarnaast lieten we ook zien dat er geen reductie was in activiteit in de verwachte hersengebieden bij het zien van lekkere calorierijke voeding na een food cue exposure therapie. Onze bevindingen suggereren dat activiteit in het mesocorticolimbische systeem meer zegt over motivationele saillantie dan over de belonende waarde van calorierijk eten. En een ander interessante conclusie is dat de effecten van mindset op neurale en hormonale responsen niet samen hoeft te hangen met daadwerkelijk eetgedrag. Verder kan geconcludeerd worden dat een indirecte en niet taak-relevante mindsetmanipulatie al kan resulteren al in een verandering van eetgedrag en zin in 
eten, maar niet in fysiologische veranderingen. Ons onderzoek onderstreept het belang van een goed gecontroleerde fMRI taak om mentale processen vast te leggen, waarbij rekening gehouden wordt met iemands huidige mindset. 
IMPACT ADDENDUM 


\section{Research findings}

The aim of the current dissertation was to examine the influence of mindset on psychological and physiological variables involved in eating behavior. Taken together, the research in this dissertation shows that mindset affects brain activity when people view food pictures, but only when the manipulation of the mindset is strong and taskbased. For example, when people who are overweight were asked to pay attention to the tastiness of the presented high caloric food pictures (hedonic mindset) neural responses were stronger than when people payed attention to the colours of the food pictures (neutral mindset). Importantly, whereas neural activity differed between these mindsets, no significant difference in neural activity was observed between highly palatable versus highly unpalatable food items. The distinction between palatable and unpalatable food items could only be made when analyzing the brain data using a multivariate analysis approach assessing the multivoxel patterns of neural activity. That is, multivoxel patterns related to palatable foods differed significantly from those related to unpalatable foods, a finding which was most pronounced for the hedonic mindset. Mindset also influenced actual eating behavior and food craving. People consumed more food and desired food more when in an induced loss of control as compared to a contro/ mindset. We did not observe any effects of mindset on metabolic and hormonal responding and the accompanying craving, hunger and satiety states. In addition, this dissertation showed that a food cue exposure intervention did not lead to any of the expected effects on brain activity as reaction to viewing visual food stimuli while in a hedonic mindset.

\section{Relevance}

Obesity is a worldwide problem; the number of adults with obesity (BMI $\geq 30 \mathrm{~kg}$ / m2) has nearly tripled since 1975 . As the population with obesity is at high risk of health complications, and is often unsuccessful at dieting and maintaining weightloss, examining mechanisms that contribute to the maintenance of obesity and/or an unhealthy lifestyle is highly important. Obesity is ultimately caused by a prolonged energy imbalance: the number of calories consumed exceeding the number of calories expended. An often-cited cause for this energy imbalance is our current obesogenic environment. However, we all live in the same environment, but not everyone is obese. It has been proposed that people who are overweight or obese may be more sensitive to this obesogenic environment. In addition, we believe that examining a 
person's current mindset (hedonic versus health/neutral) is of major importance to understand the psychological and physiological mechanisms in eating behaviour.

In this dissertation, we show that mindset is important to consider when examining psychological and physiological mechanisms in eating behaviour. Our findings lead to novel insights and more elaborative scientific understanding in the current inconsistent field of neural and cognitive mechanisms in food cue processing. Our findings show that brain processes in food perception are dynamic and dependent on someone's current mindset. Interestingly, whereas mindset did influence neural responding to visual food processing, no difference in neural activity between highly palatable versus highly unpalatable food items were found. These findings reflect a significant shift in the field, as it is commonly assumed that the level of neural activity in the mesocorticolimbic system of the brain reflects the reward value of food. Our findings suggest that this level of neural activity more likely reflects saliency. Our findings also indicate that it is difficult to grasp and change a mindset to change eating behaviour, that a mindset should be very strong and eating contextdependent.

The findings of this dissertation are valuable for society as they underline the importance of mindset for eating behaviour, that it matters which mindset you have when you engage with food. We show that a mindset influenced brain responses when seeing food items and actual eating. This knowledge could be used in daily life by helping people to have a healthy mindset when this is needed. For example, by nudging people at the right moment to make a healthier choice in a relevant context. This could be achieved by sending mindset messages to people's smartphone on relevant moments, such as when shopping for food items in a grocery store. Knowledge on the impact of mindset on the psychology and physiology of eating behaviour is essential for the improvement of interventions for reducing overweight and increasing health.

\section{Target group}

The target groups of our research are people who are coping with eating and weight problems, like people who overeat and are (at risk to become) overweight or obese. However, our research is also of relevance for people with other eating-related problems, like people suffering from bulimia nervosa or anorexia nervosa. Obviously, 
for people being underweight (i.e. due to anorexia) a different mindset manipulation target is needed than for people who are overweight or obese to improve healthy eating. In addition, the mindset manipulations used in this dissertation could also be adapted to other age groups, like adolescents and children. Furthermore, the research findings of this dissertation are of relevance to all people interested in mindset, eating behaviour, and neuroscience related to food perception, more fundamental neuroscience, and/or biological mechanisms involved in food processing. These can be people from the general population interested in (one of) these topics or people working in food science, such as dieticians, clinicians, and of course people working in academic research related to this field. If we understand more about the role of mindset and the mechanisms involved in eating behaviour, eventually treatment could be optimized by targeting problem factors better. This dissertation is also of interest for neuroscientists in general, as our research underlines the importance of a well-controlled fMRI paradigm. This to be sure of the exact ongoing mental process of participants while they perform the task in the scanner, to overcome the problem of reverse inference.

\section{Activity}

The findings of this dissertation are of great value for science. Results of the research of this dissertation have been presented at several scientific interfaculty, national and international symposia and conferences. Researchers, students and/or clinical therapists attended these conferences, and shared their thoughts and feedback on this topic. Scientific articles derived from the research from this dissertation are published or about to be published in international peer-reviewed journals.

Our research findings can give future directions for intervention development by using mindset messages to improve healthy behaviour. Our research shows that to most effectively change eating behaviour, mindset should be addressed in the right manner and in the right eating-context. One of the possibilities to do this is to design a mindset-changing mobile smartphone application, and to examine eating behaviour outcomes. This idea has already been implemented in a current running study to test the influence of mindset messages (hedonic versus health) on snacking behaviour in daily life. This is performed by using an Ecological Momentary Intervention (EMI) app for two weeks in female healthy-weight students. A next step would be to develop and use this kind of mobile application in a clinical population. 
Another activity performed to share our research findings was via teaching at Maastricht University. Teaching included lectures on topics of this dissertation and the design of teaching materials for related courses (psychology of eating, eating behaviours) for bachelor and master students in psychological science, health and social sciences and university college Venlo. Several students have joined projects from this dissertation and gained experience in executing research by assisting research, doing internships and/or writing bachelor or master theses on topics related to this dissertation.

It is also important to inform the more general public. The eating disorders and obesity eating group has organized a public event in which the research of this dissertation (and other research from our research group) was presented to the general public. Here, we provided an interactive lecture about the topics of this dissertation. We will continue to share our research on multiple (online) channels for researchers, students, clinicians and general public. 
DANKWOORD 
Na alle jaren van hard promotie-onderzoeken, is het eindresultaat dan toch echt klaar om naar de drukker te gaan: mijn proefschrift! Wat een fantastische leuke, dynamische en leerzame tijd is mijn promotieperiode geweest. Dit heb ik mede aan een heleboel geweldige mensen te danken, die ik graag op deze manier even wil benoemen.

Allereerst Anne, eerste promotor en super-supervisor! Ik durf rechtuit te zeggen dat het zonder jouw hulp nooit een succes geworden was. In alle fases van het project was je bijzonder betrokken en stond je altijd klaar met goed advies, raad en daad. Jij wist mij bij alle omstandigheden, wetenschappelijke, maar ook persoonlijke, altijd een goed gevoel te geven. Bedankt voor de geruststelling, het vertrouwen en de vrijheid die je me gaf tijdens het project. Ik vind het echt tof dat ik je inauguratie tot professor van dichtbij mee heb mogen maken. Ik bewonder je enorm: jij hebt pas écht een hoog power-vrouw gehalte! Ik ben blij dat we al zoveel jaren zo ontzettend goed samenwerken en ik heb ontzettend veel van jou geleerd. Veel dank voor alles, ik had me geen betere supervisor kunnen wensen!

Anita, mijn toffe tweede promotor. Ook jij heel erg bedankt voor alle hulp, positivisme en je kritische blik. Jij wist altijd op een juiste manier de knop door te hakken of het iets bondiger te schrijven, als Anne en ik het niet zeker wisten. Bedankt voor dat je het balletje opgooide om Yale te bezoeken voor een onderzoekvisite bij Dana Small's lab, daar heb ik veel geleerd. Ik kan me nog goed de sollicitatierondes voor dit project herinneren. Toen ik je leerde kennen, dacht ik gelijk: "Dat is een toffe professor, die mag ik wel!" Je zocht een schaap met 5 poten, ik hoop dat ik me als een voorbeeldig 5-potig schaap heb gedragen.

Dan mijn leukste paranimfen ever Romy en Astrid! Dank jullie wel voor alle steun en de gekkigheid die af en toe nodig was tijdens mijn promotietraject. Jullie kennen mij het beste in de werk-gerelateerde setting, maar ook - en zeker niet minder belangrijk - daarbuiten.

Romy, jij en ik lijken stiekem erg veel op elkaar. We leerde elkaar kennen bij start van onze bachelor en na de beruchte gezamenlijke-wiskunde-practicumin-het-computerzaaltje-met-een-gedeelde-mening-over-de tutor-ervaring was het echt "aan". We hebben vervolgens ontzettend veel dingen meegemaakt en onze vriendschap werd hechter en hechter. Jij hebt hetzelfde opleidingstraject doorlopen en we gaan vaak op dezelfde manier om met situaties. Daardoor konden en kunnen we dus altijd lekker met elkaar discussiëren, beetje zeuren of juist onze succesjes 
vieren. Al wonen we nu zo'n 225 km uit elkaar, ik ben blij dat onze vriendschap nog steeds even hecht is en dat je er altijd voor me bent. Je bent de beste!

Astrid, jij was een maand later begonnen als promovenda bij CPS. Na de "hallo" was er gelijk een klik. Wat hebben wij gelachen op de uni, maar ook daarbuiten. Koffietjes tussendoor als pauze waren door jou een feest en vrij-mi-bo's die veel langer duurde dan de mi hielpen ook om na een harde werkweek te ontspannen. Daarnaast heb ik ook ontzettend veel aan jouw steun en advies gehad wanneer ik aan het stressen was of iets beetje spannend vond. We hadden al snel besloten dat wij elkaars paranimf gingen zijn en ik kan niet wachten om die van jou te zijn! Ook nog een blijk van dank dat je mijn vaste gezellige logeeradresje was voor als ik in Maastricht (soms onverwachts) bleef slapen. Heel erg bedankt dat we samen dit PhD avontuur hebben mogen beleven en dat je zo'n goede en lieve vriendin bent!

Dan een dikke dankjewel voor alle leden van de eetgroep: Anita, Anne, Carolien, Sjaan, Sandra, Katrijn, Jessica, Ghislaine, Lotte, Pimpini, Sarah, Bart, Eric, Kamilah, Yu, Yi, Dãrta, Hanna, Michelle, Alberto en Stefanie. Maar ook voor de ex-leden van de eetgroep: Remco, Karolien, Peggy, Fania, Bastiaan, Iris, Anouk, Valerie Shannon, Clare en Vanessa. Bedankt voor de feedback en gezelligheid tijdens de Eatmeets, gedeelde symposia of congressen, borrels, leuke Braindays of gewoon zo even tussendoor. Het is erg fijn om bij zo'n mooie groep gedreven onderzoekers en therapeuten te horen. Jullie zin allemaal stuk voor stuk hele fijne mensen! Enne Sjaan, ik hoop ZO dat ik je binnenkort voor het eerst in een jurk ga zien!

Graag wil ik de leden van de beoordelingscommissie bedanken voor het lezen en beoordelen van mijn proefschrift en voor het deelnemen in mijn corona: Prof. Rainer

Goebel, Prof. Susanne la Fleur, Prof. David Linden, Prof. Liesbeth van Rossum en Prof. Annemie Schols.

Tanja, heel erg bedankt voor al je advies en hulp voor de opzet en analyses van de hormoon uitkomstmaten. En voor de vele keren dat ik mocht oefenen op jou (en Matthijs) met infuus zetten. Kelly, ook jij bedankt voor het meedenken bij het opzetten van de erg ingewikkelde studie!

Thank you a lot Dana and your team, for the possibility to visit your lab at Yale University. It was a very nice experience, I learnt a lot and I am happy we are still working together on the manuscripts. 
Also, I would like to say a big thank you to Moira and Kirsten, for the lovely time we had in New Haven, all as visiting researchers, but definitely also for the afterNew-Haven-time (London, Iceland!

Bedankt alle (ex)kamergenootjes voor de gezelligheid in "office A3.573" (ik moest het kamernummer alweer opzoeken): Fania, Iris, Anke, Kamilah en Hanna:

Fania, bij het opstarten van mijn promotietraject was jij als kamergenoot ook gelijk mijn helpende hand. Enorm bedankt voor al die keren dat je me opgevangen hebt toen het nodig was. Bedankt dat ik je paranimf mocht zijn. Ik voelde me zeer vereerd en was supertrots op hoe goed je dat deed. Ik bewonder jouw gestructureerde manier van werken en altijd goede humeur. Ik vind het ook heel mooi om je nu als mama van Lily te zien. We houden de koffietjes drinken samen erin!

Iris, jij bent zo wijs en de rust zelve. Bedankt dat je af en toe een therapeut voor me kon zijn, dat had ik af en toe wel nodig als ik door de bomen weer even het bos niet zag. Ik vond het erg jammer dat je de eetgroep ging verlaten, maar ook zo dapper dat je wist wat je wilde en daarvoor ging. Bedankt voor de leuke tijd samen als paranimf, vond ik erg gezellig!

Anke, toen jij als nieuwe roomie kwam, voelde dat gelijk goed. Bedankt voor alle goede gesprekken en voor het brengen van nog meer gezelligheid in onze snoepoffice! Hopelijk kunnen we elkaar weer snel live zien.

Kamilah and Hanna, thanks for your support and making the office as well even gezelliger and sweeter by filling our candy jar!

I would also like to thank other (FPN/(CPS/(PhD colleagues, and especially Astrid,

Matheus, Thomas, Kristoff, Evaliina, Johannes, Juliane, Kai and Rena, thanks for all the nice work and non-workrelated conversations during lunch and coffeebreaks, vrij-mi-bo drinks, EPP, or other occasions! You made my PhD-time at the UM truly amusing!

Dan zou ik graag nog studenten en andere collega's willen bedanken:

Bedankt voor alle studentassistenten en stagiaires die meegeholpen hebben aan verschillende onderdelen van mijn proefschrift. Clara, you were the best studentassistant anyone could hope for, thanks a lot for all your help, which you did with great dedication and perfectionism. I am looking forward to finalizing the 
manuscripts with you. Speciale dank aan mijn dreamteam Kyra en Vincent, zonder jullie hulp was dat ingewikkelde project niet mogelijk geweest. Wat was dat een leuk project door de motivatiefilmpjes, liedjes maar ook door de toewijding die jullie hadden!

Bedankt Scannexus, voornamelijk: Job, Esther en Chris voor de goede service en hulp bij al die scanuren die ik gehad heb. Job, super bedankt voor alle hulp met analyses en het enorme gedoe met de 7T data en ook alle goede gesprekken daarnaast! Alard, ook jij bedankt voor je scan-advies!

Bedankt dames van het (ex)secretariaat voor de gezellige gesprekjes en het goede regelwerk: Jessie, Marionne, Carolien, en Lindy. Vooral Jessie, bedankt voor al het geregel met mijn verblijf in USA en al het bestelwerk en dat je altijd lacht om mijn flauwe grapjes. Je bent een topper!

Bedankt mannen van de instrumentatie: Charlie, Michiel, Richard, Jacco, René en Erik. Ik geloof dat ik bij ieder van jullie weleens een keer (of vaker) het kantoor binnenstormde met een directe hulpvraag die altijd beantwoord werd. Charlie, speciaal bedankt voor de uiteenlopende onzinnige gesprekken en dat je alle chocola altijd komt opeten op kantoor.

Liesbeth, bedankt voor het supertoffe design van het proefschrift! Dat het nu vol staat met een hoofd vol lekkere hapjes, dat is het kersje op de taart. Ben er superblij mee!

Tot slot wil ik mijn lieve vrienden en familie in het bijzonder bedanken voor er gewoon voor altijd me zijn met hun warme en toewijding. Een paar wil ik graag even in het speciaal benoemen:

Barbara, Dayinta, Jorien en Romy, samen vormde wij sinds de bachelor the “HoT GiRlszZ" (ja, het staat echt in mijn proefschrift nu, zelfs dikgedrukt). Al dik 13 jaar zijn wij een groepje van (soort van) gelijkgestemde vrouwen. Jullie inspireren me ieder op jullie eigen manier. Bedankt voor alle steun en alle mooie momenten die we hebben mogen meemaken. Ook al wonen we allemaal ergens anders en ver uit elkaar, ben ik blij dat we nog steeds een innige vriendschap hebben en dat we altijd samenkomen op belangrijke momenten. Ik hoop dat we nog jarenlang gek mogen dansen, veel gegratineerde nacho's mogen eten, weekendjes of weekjes weg kunnen, en dat er nog meer prachtige babies geboren mogen worden. $<3<3<3<3$ 
Nanet is gek, zo had ik je opgeslagen in mijn telefoon nadat ik je leerde kennen. Ik denk een grapje die alleen wij begrijpen. Bedankt voor alle ontspan en relativeer momentjes met slechte tv-avonden, dansjes, terrasjes pakken en goede diepgaande gesprekken. Bedankt dat ik altijd ongegeneerd mezelf mag zijn. En samen met Gytha en Jelske vormen wij de enige echte LHs-Club. Bedankt voor alle gezellige avondjes, hoop lekkere hapjes en fijne weekendjes weg!

Yoga Josée, ben zo blij dat we elkaar bij de yoga leerde kennen, elke week raakte we niet uitgepraat en een vriendschap ontstond snel. We spraken vaak af voor een hapje of een drankje en nu meestal voor een stevige wandeling met een goed gesprek. Bedankt dat je zo'n lieve en zorgzame vriendin voor me bent!

Steffi, bedankt dat je al zoveel jaren zo lief bent en begripvol. De ongelofelijke reis samen met jou door Maleisië zal ik nooit meer vergeten, en heb nog zo vaak moeten lachen als ik terugdacht aan verschillende momenten daar, bedankt daarvoor!

Serena en Juulke, al sinds de middelbare school zijn we vriendinnen. Ook jullie bedankt voor alle lieve steun en gezelligheid door al die jaren heen. Serena, ik ben de tel kwijt, 18 jaar vriendschap? Al woon je niet meer in Venlo en spreken we elkaar minder, ik weet dat ik maar hoef te piepen en dat je er voor me bent. Laf joe!

Familie is alles. Bedankt lieve Oma, Mama, Papa, Hannie, Marc, Thei en Valerie, Frans en Liesbeth en Lou en Jopie, Kaat en Lilo. Bedankt dat jullie er altijd zijn met al jullie liefde, drukte en gezelligheid.

Papa, bedankt dat je altijd een warm thuisgevoel creëert. Thei, bedankt voor je beschermende rol als grote broer. Frans, bedankt voor je verbindende rol als grote broer. Kaat, bedankt voor je zorgzame rol als grote zus. Lilo, Lou en Jopie bedankt voor de lach op mijn gezicht, jullie zijn geweldig, kleine boefjes!

Oma, helaas heb je het niet meer kunnen maken, maar het is eindelijk gelukt hoor! Wat was jij altijd trots om te kunnen vertellen waar ik mee bezig was en wat ben ik trots om altijd zo'n toffe oma gehad te hebben.

Mama, de allerbeste. Ik mis je elke dag. Maar ik weet dat dit proefschrift er ligt door jouw toedoen. Jij hebt mij gemaakt tot wie ik vandaag ben. Bedankt voor alle inspiratie die je me nog steeds geeft. Voor altijd in mijn hart. 
Micha, liefde van mijn leven. Je bent mijn steun en toeverlaat die altijd klaar staat met een dikke knuffel. Ik bewonder je optimistische kijk op het leven en dat je me zo goed helpt om te relativeren. Je bent gewoon zo'n mooi mens die ik (toch wel onverwachts; ; ) ) heel graag in mijn buurt heb. Samen met onze kattenvriendjes Frunnik en Bruce hebben we samen een fijn thuis gemaakt. Ik hou van je en ik heb zin in alle avonturen die we in de toekomst nog samen gaan beleven... 
CURRICULUM VITAE 
Sieske Henriëtte Margriet Jessica was born on April 15, 1988 in Venlo (the Netherlands). She graduated from the secondary school in 2007 (Atheneum, Valuas College, Venlo). She completed a bachelor of science in Beta Gamma with a major Psychobiology in 2011, followed by completing a research master (2013) in Biomedical Sciences: track Neurobiology, Cognition and Computation, both at the Faculty of Science, University of Amsterdam, Amsterdam. During the research master, she did two long internships using MRI-methods to examine human behaviour. These internships were completed at the department Radiology, Amsterdam Medical Centre (AMC) in Amsterdam and at the Donders Institute for Brain, Cognition and Behaviour / Department of Neurology, Radboud University Medical Centre Nijmegen (UMCN) in Nijmegen. After her master, she worked as commercial and technical support in computer store Tanichi in Venlo, and as a high school student tutor in physics and chemistry. In January 2015, she started her PhD project in the research group: Eating Disorders and Obesity at the Department of Clinical Psychological Science at Maastricht University. During her PhD, she studied the influence of cognitive mindsets on neural activity, hormonal and metabolic responses in eating behavior under supervision of Prof. Anne Roefs and Prof. Anita Jansen. In 2017, she visited John B. Pierce Laboratory affiliated to Yale University, New Haven (USA, CT) in the research group 'Neuropsychology and Physiology of Flavor and Feeding' chaired by Prof. Dana Small. Since March 2020, Sieske is working as post-doc in the same research group. 


\section{Publications and presentations}

\section{Published manuscripts}

Franssen, S., Jansen, A., van den Hurk, J., Roebroeck, A., \& Roefs, A. (2020), Power of mind: Attentional focus rather than palatability dominates neural responding to visual food stimuli in females with overweight. Appetite, 148, 104609. https://doi.org/10.1016/j.appet.2020.104609

Franssen, S., Jansen, A., Schyns, G., van den Akker, K., \& Roefs, A. (2020), Neural Correlates of Food Cue Exposure Intervention for Obesity: A Case-Series Approach. Front. Behav. Neurosci. 14, 1-11. doi:10.3389/fnbeh.2020.00046.

Roefs, A., Franssen, S., \& Jansen, A. (2018). The dynamic nature of food reward processing in the brain. Current Opinion in Clinical Nutrition and Metabolic Care, 21(6), 444-448.

Schaapsmeerders, P., Tuladhar, A. M., Arntz, R. M., Franssen, S., Maaijwee, N. A. M., Rutten-Jacobs, L. C. A., ... De Leeuw, F.-E. (2016). Remote Lower White Matter Integrity Increases the Risk of Long-Term Cognitive Impairment after Ischemic Stroke in Young Adults. Stroke, 47(10).

\section{Other manuscripts}

Franssen, S., Jansen, A., Adam, T., van den Hurk, J., Roebroeck, A., \& Roefs, A. Effects of mindset on hormonal responding, neural representations, subjective experience and intake (under revision at Physiology and Behavior)

Franssen, S., Roefs, A., Raithel, C., Jansen, A., \& Small, D. The effect of perceived caloric content on the metabolic response after drinking a sweetened beverage (submitted at Physiology and Behavior)

Canna, A., Cantone, E., Roefs, A., Franssen, S., Prinster, A., De Martino, F., Formisano, E., Di Salle, F., \& Esposito, F., Functional Dissection of Taste Subcortical Neural Pathway in Isolated Ageusia as a Study Model for Covid-19 Neurotropism: 7 Tesla Functional MRI of Nucleus Tractus Solitarii in Humans during Gustatory Stimulation (ready for submission) 
Kochs, S., Franssen, S., Pimpini, L., Van den Hurk, J, Roebroeck, A., Jansen, A., \& Roefs, A., It is a question of perspective: attentional focus drives neural responding to food cues (ready for submission)

Franssen, S., Jansen, A., Raithel, C., Van den Hurk, J., Geijskens, K., Adam, T., Small, D., \& Roefs, A. Your mind on sweet taste: influence of mindset on the neural correlates of taste perception (in preparation)

Desmet, M.*, Franssen, S.*, Varol, T., Fillon, A., Thivel, D., Roefs, A., \& Braet, C., The feasibility of a smartphone application as a personalized treatment tool for overweight adolescents: an explorative study (*shared irst authorship, in preparation)

Pimpini, L., ${ }^{*}$ Franssen, S., * Reber, F., \& Roefs, A., Tell me what you are snacking on: An Ecological Momentary Intervention study on the effect of mindset on snack foods' craving and consumption (*shared first authorship, in preparation)

Pimpini, L., Kochs, S., Franssen, S., Jansen., A., Van den Hurk, J. \& Roefs, A., Attention eats it all: The way you look at food determines the way your brain represents it (in preparation)

Canna, A., Roefs, A., Franssen, S., Prinster, A., Cantone, E., Di Salle, F.Formisano, E., \& Esposito, F., Is there a specialization for taste quality in Primary Gustatory Cortex: A 7 Tesla fMRI study (in preparation)

\section{Presentations at (inter)national conferences and symposia}

Franssen, S., Jansen, A., van den Hurk, J., Roebroeck, A., \& Roefs, A., (2019, July), Power of mind: Attentional focus rather than palatability dominates neural responding to visual food stimuli in females with overweight. Poster presentation at the 27th Annual Meeting of the Society for the Study of Ingestive Behavior (SSIB), Utrecht, the Netherlands

Franssen, S., Jansen, A., van den Hurk, J., Roebroeck, A., \& Roefs, A., (2019, April), Power of mind: Attentional focus rather than palatability dominates neural responding to visual food stimuli in females with overweight. Poster presentation Poster presentation at the Research Day of the faculty of Psychology and Neuroscience (FPN), Maastricht, the Netherlands 
Franssen, S., \& Roefs. A., (2018, October), Power of mind. Oral Presentation on PhD Project at the Dutch-Flemish postgraduate school of Experimental Psychopathology (EPP) symposium Empathy and Theory of Mind, Heeze, the Netherlands

Franssen, S., Jansen, A., van den Hurk, J., Roebroeck, A., \& Roefs, A., (2018, June), Power of mind: Attentional focus rather than palatability dominates neural responding to visual food stimuli in females with overweight. Poster presentation at Dutch Neuroscience Meeting 2018, Lunteren, the Netherlands

Franssen, S., (2018, April), Power of mind. Oral presentation at the FPN Research Day, Maastricht University, Maastricht, the Netherlands

Franssen, S., (2017, March), Power of mind. Oral presentation for Battle of the Brains at Annual EPP day, Utrecht, The Netherlands

Franssen, S., (2016, November), Oral presentation PhD project progress at the Eatwell Workshop, Maastricht, The Netherlands

Franssen, S., \& Roefs. A., (2016 June), Oral presentation: eating and the power of mind at the information day on psychology of eating for general public, Geleen, The Netherlands

Franssen, S., Jansen, A., Adam, T., van den Hurk, J., Roebroeck, A., \& Roefs, A., (2016, April), Poster presentation: Once you pop, you can't stop at the FPN Research Day, Maastricht, The Netherlands

Franssen, S., Jansen, A., Adam, T., van den Hurk, J., Roebroeck, A., \& Roefs, A., (2015, October), Oral presentation on research progress of the project: once you pop, you can't stop at the Dutch Research Council / Nederlandse Organisatie voor Wetenschappelijk Onderzoek (NWO) Food Brain Cognition site visit at FPN, Maastricht, The Netherlands

Franssen, S., \& Roefs. A., (2015, March), Oral presentation: 3T MRI project proposal at the fMRI and NIBS (Non Invasive Brain Stimulation) project proposal meeting at FPN, Maastricht, The Netherlands 
